\title{
DEVELOPING EFFECTIVE PROFESSIONAL LEARNING IN CAMBODIA
}

Jan B. Y. Berkvens 
DOCTORAL COMMITTEE

Chairman: $\quad$ Prof. dr. H. W. A. M. Coonen "University of Twente

Promoters: $\quad$ Prof. dr. J. J. H. van den Akker v University of Twente

Prof. dr. W. A. J. M. Kuiper * University of Utrecht

Members: $\quad$ Prof. dr. J. M. Pieters * University of Twente

Prof. dr. J. W. M. Kessels v University of Twente

Prof. dr. M. Kalyanpur v Towson University, Baltimore, USA

Dr. W. J. W. Ottevanger v VU University Amsterdam

Berkvens, Johannes Bernardus Yvo.

Developing effective professional learning in Cambodia

Thesis University of Twente, Enschede.

ISBN 978-90-365-2861.0

DOI $10.3990 / 1.9789036528610$

Cover: Nienke Haccou

Layout: Sandra Schele

Press: PrintPartners Ipskamp - Enschede

(C) Copyright, 2009, Jan B. Y. Berkvens

All rights reserved. No part of this book may be produced in any form: by print, photocopy, microfilm, or any other means without written permission from the author. 
DEVELOPING EFFECTIVE PROFESSIONAL LEARNING IN CAMBODIA

\author{
DISSERTATION
}

\author{
to obtain \\ the degree of doctor at the University of Twente \\ on the authority of the rector magnificus \\ prof. dr. H. Brinksma \\ on account of the decision of the graduation committee \\ to be publicly defended \\ on Wednesday the 10 $10^{\text {th }}$ of September 2009 at 16.45
}

by

Johannes Bernardus Yvo Berkvens

born on the $1^{\text {st }}$ of April 1972

in Schiedam, the Netherlands 
Promoters:

Prof. dr. J.J.H. van den Akker

Prof. Dr. W.A.J.M. Kuiper 
'This is the place I want to stay

I love the rhythm, the long slow day

The evening time when the boats slide by

This is the place I want to stay

The joy is so sharp and so is the sadness'

'The place I want to stay', from 'Where elephants weep'

(A Khmer Rock Opera, Filloux, 2008)

For Cambodia and the ones I love 



\section{TABLE OF CONTENTS}

LIST OF FIGURES AND TABLES vii

$\begin{array}{ll}\text { PREFACE ix } & \text { ix }\end{array}$

1. INTRODUCTION 1

1.1 Origin of the study 1

1.2 Need for the study 1

1.3 Context of the study 3

1.3.1 The Kingdom of Cambodia 3

1.3.2 Education in Cambodia 5

1.3.3 The Ministry of Education, Youth and Sports 6

$\begin{array}{ll}\text { 1.3.4 Implications for the study } & 7\end{array}$

1.4 Aim of the study and research question 8

1.5 Overview of following chapters 9

2. RESEARCH APPROACH AND DESIGN

$\begin{array}{lll}2.1 & \text { Research approach } & 11\end{array}$

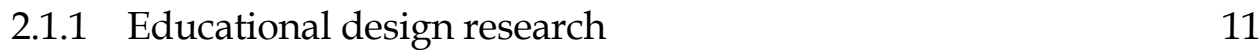

2.1.2 Validity and reliability of educational design research 12

2.1.3 Quality criteria for products and scenarios 13

$\begin{array}{ll}2.2 \text { Research design } & 13\end{array}$

2.2.1 The research design phases 13

$\begin{array}{lll}\text { 2.2.2 The preparatory phase } & 14\end{array}$

2.2.3 The design and implementation phase 16

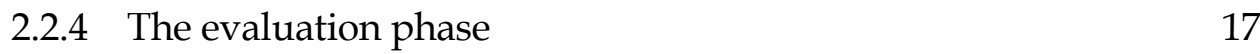

2.2.5 Data collection 20

2.3 Minimizing biases 21

$\begin{array}{lll}2.3 .1 & \text { Role bias } & 21\end{array}$

2.3.2 Insider/outsider bias 22

2.3.3 Cultural Bias $\quad 22$

2.3.4 Response bias $\quad 23$

$\begin{array}{ll}2.3 .5 & \text { Selection bias } \\ & 23\end{array}$ 
2.4 Limitations of the study 23

2.4.1 Small sample size and absenteeism 23

$\begin{array}{ll}2.4 .2 & \text { Limited generalisability } 24\end{array}$

3. CONTEXT ANALYSIS $\quad 25$

3.1 Introduction 25

3.2 The history of Cambodian education 27

3.2.1 Education before and during the French protectorate and early independence 27

3.2.2 Education during the Khmer Rouge era 32

3.2.3 Education during and after Vietnamese occupation 35

3.2.4 Cambodia in the globalised world and the demands for education 41

3.3 Culture and professional development 42

3.3.1 War and the complexity of Cambodian culture 42

3.3.2 The 5-D model for cultural differences 44

3.3.3 The influence of culture on learning 51

3.3.4 Learning in a changing society 53

3.4 Professional development in Cambodia 57

3.4.1 Aid and professional development $\quad 57$

$\begin{array}{ll}3.4 .2 & \text { Donor influence }\end{array}$

3.4.3 Professional development in practice $\quad 65$

3.4.4 Training and workshops in practice 66

$\begin{array}{lll}3.5 & \text { The need for change } & 69\end{array}$

3.6 Implications for a more effective professional development $\begin{array}{ll}\text { scenario } & 71\end{array}$

$\begin{array}{ll}\text { 4. LITERATURE REVIEW } & 77\end{array}$

$\begin{array}{lll}4.1 & \text { Introduction } & 77\end{array}$

$\begin{array}{lll}4.2 & \text { Human resource management } & 79\end{array}$

$\begin{array}{lll}\text { 4.2.1 HRM in developing countries } & 79\end{array}$

4.2.2 The need for a more integral approach of HRM 80

4.2.3 Power issues in local HRM systems $\quad 81$

4.2.4 Support issues in local HRM systems $\quad 83$

4.2.5 Design principles from the HRM perspective 84

$\begin{array}{lll}4.3 & \text { Professional development } & 85\end{array}$

4.3.1 Professional development and learning 85 
4.3.2 Empowering people to learn

4.3.3 Design principles from the professional development perspective $\quad 90$

4.4 Curriculum development 90

$\begin{array}{lll}\text { 4.4.1 A consistent curriculum } & 90\end{array}$

$\begin{array}{lll}\text { 4.4.2 A corporate curriculum } & 91\end{array}$

4.4.3 Design principles from the curriculum development perspective $\quad 93$

4.5 Adult learning 94

4.5.1 Characteristics of adult learning 94

4.5.2 Factors influencing adult learning 95

4.5.3 A socio-constructivist approach to learning 98

$\begin{array}{lll}\text { 4.5.4 Experience and learning } & 100\end{array}$

4.5.5 Reflection and learning 100

$\begin{array}{ll}\text { 4.5.6 Collaborative learning } & 104\end{array}$

4.5.7 Jarvis's model of adult learning 106

4.5.8 Cognitive apprenticeship to support learning 107

4.5.9 Design principles from the adult learning perspective 108

$\begin{array}{ll}4.6 \text { Culturally relevant learning } & 109\end{array}$

4.6.1 The necessity for culturally relevant learning 109

4.6.2 Design principles from the cultural relevant learning $\begin{array}{ll}\text { perspective } & 111\end{array}$

5. PORTRAYING THE ENACTED SCENARIO 113

$\begin{array}{lll}5.1 & \text { Introduction } & 113\end{array}$

$\begin{array}{lll}5.2 & \text { The start of the scenario } & 117\end{array}$

5.2.1 Situating the scenario in the Ministry 117

5.2.2 Characteristics of the community of learners 118

5.2.3 Characteristics of training endeavours and workshops 124

$\begin{array}{ll}\text { 5.2.4 Conclusion at the start of the scenario } & 126\end{array}$

$\begin{array}{lll}5.3 & \text { The components of the scenario } & 127\end{array}$

$\begin{array}{ll}\text { 5.3.1 The working group meetings } & 127\end{array}$

5.3.2 The preparatory meetings 134

$\begin{array}{ll}\text { 5.3.3 The four-day training } & 137\end{array}$

$\begin{array}{ll}5.4 & \text { Learning during the scenario } \\ & 139\end{array}$

5.4.1 Steps in learning 139

$\begin{array}{lll}\text { 5.4.2 Taking learning a step further } & 140\end{array}$ 
5.4.3 The importance of micro-cycles

5.4.4 Strengthening meta-cognitive, regulative and communication skills

143

5.4.5 Conclusion

6. EVALUATION OF THE ENACTED SCENARIO $\mathbf{1 4 5}$

$\begin{array}{lll}6.1 & \text { Introduction } & 145\end{array}$

$\begin{array}{lll}6.2 & \text { Instruments and procedures } & 146\end{array}$

$\begin{array}{ll}\text { 6.3 Evaluation of the scenario as a curriculum } & 147\end{array}$

$\begin{array}{lll}6.3 .1 & \text { Rationale } & 147\end{array}$

6.3.2 Aims and objectives 149

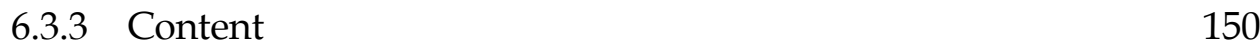

6.3.4 Learning activities 152

6.3.5 The role of the facilitator 155

$\begin{array}{ll}\text { 6.3.6 Materials } & 158\end{array}$

$\begin{array}{lll}\text { 6.3.7 Grouping } & 161\end{array}$

$\begin{array}{lll}6.3 .8 & \text { Location } & 163\end{array}$

$\begin{array}{lll}6.3 .9 & \text { Time } & 154\end{array}$

$\begin{array}{ll}\text { 6.3.10 Assessment } & 168\end{array}$

6.3.11 Consistency of the curriculum 169

6.4 Effectiveness of the scenario as a professional development $\begin{array}{ll}\text { activity } & 169\end{array}$

6.4.1 Participants' reactions 169

$\begin{array}{lll}\text { 6.4.2 Participants' learning } & 171\end{array}$

$\begin{array}{lll}\text { 6.4.3 Organisational support } & 173\end{array}$

$\begin{array}{lll}\text { 6.4.4 Participants' use } & 174\end{array}$

$\begin{array}{lll}6.4 .5 & \text { Secondary beneficiaries } & 178\end{array}$

6.4.6 Overall effectiveness of the scenario 179

6.5 Cultural influences on the scenario 180

6.5.1 The first cultural dimension: collectivist versus individualist $\quad 180$

6.5.2 The second cultural dimension: power distance 181

6.5.3 The third cultural dimension: uncertainty avoidance 183

6.5.4 The fourth cultural dimension: femininity versus masculinity 185

6.5.5 The fifth cultural dimension: long term versus short term orientation 
6.5.6 Overall influence of culture on the scenario 188

$\begin{array}{ll}6.6 \text { Conclusion } & 189\end{array}$

$\begin{array}{ll}\text { 7. DISCUSSION } & 193\end{array}$

$\begin{array}{lll}7.1 & \text { Recapitulation of the study } & 193\end{array}$

7.1.1 Research context and question 193

$\begin{array}{lll}\text { 7.1.2 Preparation of the scenario } & 194\end{array}$

7.1.3 Design and implementation of the scenario 196

7.1.4 Evaluation of the enacted scenario 196

$\begin{array}{ll}\text { 7.1.5 Main findings } & 197\end{array}$

7.2 Discussion of the main findings 200

$\begin{array}{lll}7.2 .1 & \text { Evaluation criteria } & 200\end{array}$

7.2.2 The necessity of a shared conceptual framework 200

7.2.3 The need for a safe learning environment 202

7.2.4 The collaborative 'learning by designing' approach 202

7.2.5 The threats of a participatory approach 203

7.2.6 Support to learning 205

$\begin{array}{lll}7.2 .7 & \text { Facilitator typology } & 207\end{array}$

$\begin{array}{lll}7.3 & \text { Reflection on the research methodology } & 209\end{array}$

$\begin{array}{lll}\text { 7.3.1 The preparatory phase } & 210\end{array}$

7.3.2 The design and implementation phase 211

$\begin{array}{ll}\text { 7.3.3 The evaluation phase } & 213\end{array}$

$\begin{array}{lll}7.4 & \text { Reflection on the scenario } & 214\end{array}$

$\begin{array}{lll}7.5 \text { Conclusions and recommendations } & 215\end{array}$

$\begin{array}{lll}7.5 .1 & \text { Conclusions } & 215\end{array}$

$\begin{array}{lll}\text { 7.5.2 Recommendations } & 218\end{array}$

$\begin{array}{lr}\text { REFERENCES } & 225\end{array}$

$\begin{array}{ll}\text { ENGLISH SUMMARY } & 235\end{array}$

$\begin{array}{ll}\text { DUTCH SUMMARY } & 243\end{array}$

$\begin{array}{lr}\text { ANNEXES } & 251\end{array}$

1. Directors' interview letter 251

2. Directors' interview questionnaire 253

3. Training guideline explanation sheet 257 
4. First interview questionnaire 259

5. First interview successfulness 263

6. Final interview questionnaire 269

7. List of analysis codes 271

8. Short-term evaluation form first and second prototypes 273

9. Long-term evaluation form first and second prototypes 275

10. Short-term evaluation form final version 277

11. Long-term evaluation form final version $\quad 279$

12. Evaluation, monitoring and follow-up framework 281

13. Flipchart 'paon roboch knjom' 283

14. Checklist 1 first prototype 285

15. Checklist 2 first prototype 287

16. Activity form 289

17. Categorisation icebreaker/energizer/wrap-up 291

18. Checklists 293

19. Definitions monitoring/evaluation/follow-up first prototype 297

20. Definitions monitoring/ evaluation/follow-up final version 299

21. Training guideline schedule 301

22. Certification letter 303

23. 'The quest' list 305

24. Participatory level check 307 


\section{LIST OF FIGURES AND TABLES}

\section{FIGURES}

2.1 Overview of the three phases of the research design 14

2.2 Curricular Spider web 20

3.1 Different priorities of societies 53

4.1 Kolb cycle of reflection with socio-constructivist notions 101

4.2 Cowan's model of reflection 102

$\begin{array}{lll}4.3 & \text { Jarvis's model for learning } & 107\end{array}$

5.1 The place of the scenario design and implementation in the $\begin{array}{ll}\text { research } & 114\end{array}$

$\begin{array}{lll}\text { 6.1 Overview of the evaluation phase } & 145\end{array}$

\section{TABLES}

2.1 Guskey's five critical levels of professional development $\begin{array}{ll}\text { evaluation model (adapted) } & 19\end{array}$

3.1 Number of students in Cambodia per kind, 1930-1960 29

3.2 Enrolment percentages for primary education in 2002 from EMIS 39

3.3 Adjusted overview of human institutions and corresponding role pairs or learning relationships $\quad 51$

5.1 The components of the scenario, including content and data $\begin{array}{ll}\text { gathering tools } & 116\end{array}$

$\begin{array}{lll}7.1 & \text { Typology of facilitators } & 208\end{array}$ 


\section{PREFACE}

The research presented in this dissertation was carried out during my assignment at the Ministry of Education, Youth and Sports in Cambodia, from 2006 to 2009. Many people have contributed to this research in many different ways and from many places in the world. I am grateful to all the people who, in one way or another, contributed to this research, or supported my work.

In Cambodia, I would like to thank His Excellency the Director General Koeu NayLeang and the Deputy Director of the DGE Leang Nguonly for allowing me to work with their staff for the duration of this research. I am greatly indebted to my colleagues and members of the working group, Mr. Nuon Vansoeun, Ms. Chhay Kim Sotheavy, Mr. Eng Kimly, Ms. Tan Ly Huong, Mr. Tep Chhay, Mr. Ly Sokbora, Ms. Plong Mony Kosal, Mr. Sok Sokhom, Mr. Chap Kim Chun, Mr. Chin Nivath, Mr. Chhan Bunthan, Ms. Chhun Sok Yean and Mr. Nuon Bunthan with whom this research was conducted. Their willingness to answer all my questions and their openness provided me with insights that would otherwise have been impossible to acquire. Thank you for the inspirational time you gave me and all the ceremonies, weddings and gatherings you invited me to.

I am also thankful to the staff of Voluntary Service Overseas, both in the Netherlands and Cambodia, for offering me the possibility of working in Cambodia and conducting my research at the same time. The staff of the Cambodian office, especially Mr. Chea Vantha, was extremely helpful to me and answered all my queries. Great teaching by Mr. Than Dara was crucial for the acquisition of the Khmer language. Mr. Hiroyuki Hattori, Mr. Chea Huot and Mr. Tim Phalmean from UNICEF were also very supportive towards this research. I would also like to thank Ms. Mary Dunbar for the fruitful discussions we had about Cambodian culture and learning.

I am heavily indebted to my Cambodian friends, who allowed me to be part of their daily lives, and offered me the opportunity to understand Cambodian history and culture from the inside out. Without their great friendship, I would not have 
been able to acquire their language or experience their culture. I would like to specifically mention Khan Boline, Hun Touch, Suong Sereivin, Ou Lacana and Dos Sovanak for their precious friendships. My sincere thanks go to Catherine Filloux, for allowing me to use two verses from the libretto of the first Cambodian opera 'Where elephants weep'.

I am most grateful to Maya Kalyanpur for her friendship, support and for constantly challenging my academic thinking. Thank you, not only for your continuous support, keeping me motivated, and protection from academic pitfalls, but also for introducing me to the masala dosai.

In the Netherlands, my sincere thanks go to my supervisors Jan van den Akker and Wilmad Kuiper for their support during this PhD trajectory. Their method of questioning my writings stimulated my academic thought and not only shaped this research, but also shaped my academic attitude. I valued every single one of our meetings, and remember them as fruitful and pleasant. The lunches were also greatly appreciated!

I would also like to thank Sandra Schele for taking care of the layout and further organisation of this booklet. I am also grateful to Nienke Haccou for designing the cover, and Ineke Mathot for advising me on the Dutch version of the summary.

And of course, I would like to thank my friends and family for supporting me and taking me out, when I was heading towards complete isolation, especially Sandra Otten, who has become one of my most precious friends over the last few years, and, of course, Floris Smit.

In England, I would like to thank Freda Ellis and Jane Thompson for their greatly appreciated contribution to my English. Their questions about the intended meaning behind some of my syntax opened my eyes to the blanks I could not see anymore. I have finally started to grasp how to use colons, hyphens and other punctuation marks! And, of course, thank you for the wonderful hikes we made throughout Dorset during our 'revision weekends'.

Overall I would like to thank everybody who has contributed to my education over the years. Without their input, I would never have been able to complete this piece of work. Most of all, I remain indebted to my mother, for her ever-present support and never-diminishing belief in me.

Amsterdam, 25 May 2009. 



\section{CHAPTER 1}

\section{Introduction}

\subsection{ORIGIN OF THE STUDY}

This research reflects the work of the researcher as a capacity building and learning adviser at the Directorate General of Education (DGE) of the Ministry of Education, Youth and Sports (MoEYS) in Cambodia, from February 2006 to December 2008. The placement at the Ministry was a position financed by Voluntary Service Overseas (VSO) and UNICEF Cambodia, and aimed at strengthening the capacity building of staff of the DGE and its Departments in the Ministry. The Deputy Director of the DGE, together with the Education Program Managers of UNICEF and VSO, asked the researcher to investigate how the effectiveness of training courses and workshops could be improved. 'Participatory approach' and 'learner-based methodology' were key concepts asked to be included in the proposal. This request formed the basis for the research described in this dissertation.

\subsection{NEED FOR THE STUDY}

From the start of the sovereign Kingdom of Cambodia in 1993 the Cambodian Government, with help from international partners, has been trying to build the capacity of its staff in order to rebuild the war-torn country. Many professional development activities (PDAs) have been carried out over the last fifteen years to build the capacity of Government staff, using large amounts of money and human resources. In recent years, the effectiveness of these activities, which were mostly conducted as training courses or workshops, has begun to be doubted by national and international officials. There is a small report base indicating that there is valid reason for this doubt in most Government sectors, including the education sector. 
For instance, a 2004 UNESCO survey (Beynon et al., 2004), describing the effectiveness of PDAs in MoEYS concludes that most PDAs delivered to or by MoEYS are neither effective, nor sustainable. The major complaint is that Ministry staff have been the recipients of extensive training activities over the years, but the transfer of new skills, knowledge and attitudes into their daily work has been limited or non-existent. The survey provides numerous reasons why this is the case, including the weak facilitation skills of local and international trainers, lack of knowledge and skills transferred from expatriates to local staff, a high level of absenteeism from work, as well as a one-sided theoretical approach used during the activities. The survey also states that necessary organizational change and development that should be taking place with any capacity building endeavour, is seldom striven for in the Cambodian setting. The social and cultural context is identified as one of the reasons why organisational change is difficult to accomplish.

An initial context analysis for the research described in this thesis was conducted in early 2006 and confirmed the findings of the UNESCO 2004 survey. A large number of Ministry staff has followed several 'training the trainer' programmes, but the effects in terms of application are low. Interviewees claim to have had theoretical training, but no practical experimentation, which made it hard to use the newly acquired knowledge in their work. Of all the PDAs delivered to and/or by Ministry staff which were observed, only one used a participatory and learner-based approach. The existence of a systems gap was also found during the initial analysis: the Government and development partners are working on conceptual levels, developing policies and programs that do not connect with the grassroots level. Development partners especially seem to base their work on documents, without a clear understanding of the context. This systems gap is not described in any of the local documents, but is relevant for understanding the low outcomes of PDAs.

The poor quality of PDAs delivered to or by Ministry staff is a challenge for the implementation of policy the Royal Government of Cambodia has chosen to embrace, for example, its commitment to the Dakar Millennium Goal of 'Education for All' and the additional agreements it has made with aid organisations to improve education (National Education For All Committee [NEFAC], 2003). Professional development of the national staff at all levels is a necessary condition for the success of these programs in improving education. 
Guskey (2000) affirms that professional development is at the core of effective improvements in education. According to his research, effective and sustainable improvements in education almost never take place without professional development. It is this professional development that is poor in Cambodia. He also mentions the significance of a strong program design with sufficient organisational support. Joyce and Showers (2002) stress the importance of practising the skills being developed, receiving feedback and subsequent (peer) follow-up to participants of PDAs, in order to enhance transfer from the professional development setting to the daily work. All of these factors are weak in the Cambodian situation.

The Ministry of Education acknowledges that the professional development it provides is weak and therefore asked for an investigation as to why PDAs are not effective and how the effectiveness of PDAs could be strengthened. This study is the result of that request.

The following section describes Cambodia, both past and present, and the current state and structure of the education system. Section 1.4 provides the aim of the study and the research questions.

\subsection{CONTEXT OF THE STUDY}

\subsubsection{The Kingdom of Cambodia}

Cambodia is a relatively small Southeast Asian country near the Gulf of Thailand. It has a land area of 181,035 square kilometres and is enclosed by Thailand, Laos and Vietnam. Cambodia had 13.7 million inhabitants in 2005, in a country that is roughly five times the size of the Netherlands. The majority of the population lives in the countryside, but between one and two million Cambodians live in towns, of which over a million are living in the capital, Phnom Penh. Cambodia's population exists mainly of ethnic Khmer (85\%). The rest of the population is formed by the Cham (Khmer Muslim 4\%), Chinese, Vietnamese and a number of small ethnic communities living in the northeast, known as 'highland tribal people', but who belong to different tribes like the Phnong, Tomphoeun and Kroeung (Save Cambodia's Wildlife [SCW], 2006). 
Life expectancy is relatively low in the country, at 58 years for females and 54 for males (data from 2000; SCW, 2006). 60.8\% of the population is below 25 years of age, while those over 65 years of age are under-represented at only $3.7 \%$. There are more women than men, in a ratio of 6:5. These unbalanced ratios are attributed to the Pol Pot regime, which lasted from 1975 to 1979, and the long civil war that followed it (SCW, 2006).

On 17 April 1975, the Khmer Rouge army, led by Pol Pot, marched into Phnom Penh and took over control of Cambodia from the Lon Nol regime. This power shift started the 'Year Zero' era of the Khmer Rouge, with its killing fields and harsh living conditions. Within hours of taking control of Phnom Penh, the Khmer Rouge soldiers started to evacuate the city. In the space of three days almost every city-dweller was deported to the countryside to work as a farmer in the fields. In the ideology of the Khmer Rouge, a peasant society like the ancient Angkor Empire was the ultimate goal to be achieved. In such a society there was no place for intellectuals, or anything that reflected their lifestyle. Almost two million people died during the regime, including the majority of Cambodia's intellectuals and educated people (Livingstone, 1996).

During the period of Khmer Rouge control, there was no formal schooling and most educated people were systematically slaughtered. Many libraries, archives and other sources of knowledge were destroyed. Art collections did not have any value for the new rulers and were at best neglected or sold, and at worst, destroyed. Some intellectuals survived the harsh years by pretending to be uneducated in order to avoid detection and death.

On 7 January 1979, the Vietnamese overthrew the Khmer Rouge regime, after a relatively small offensive. The country was in a bad condition and soon after the start of the Vietnamese rule a famine took place: because of the turmoil after the defeat of the Khmer Rouge, there was not enough rice planted for the following season. In addition, the violence had not stopped, and civil war continued in parts of the country. This caused many people to flee to neighbouring states, with most refugees seeking refuge in Thailand. Many (of the) Cambodians lived in the refugee camps for nearly ten years, until the Paris Peace Treaty of 23 October 1991 was signed and people were encouraged to move back to Cambodia, even though some parts of the country were still not safe. According to Peterse and Petri (2004), some Khmer Rouge factions remained active until 1994, but the 1996 
Lonely Planet guide for Cambodia describes many towns, the railway system and some roads as being 'off-limits' to foreigners due to ongoing Khmer Rouge activity (Taylor, Wheeler, \& Robinson, 1996). Anecdotal evidence from some Khmers suggests that the Khmer Rouge remained active in some parts of the country as late as 1998, which corresponds with Verkoren's (2005) findings that factions remained active until that year.

At present, the Government of Cambodia, together with donor organisations and NGOs, is rebuilding the country, which is finally at peace. Although the speed of the rebuilding process is relatively fast, life is only improving slowly for the people who live in more rural areas. Development of the cities, of which Phnom Penh is in the lead, is proceeding fast. The infrastructure in and between cities is improving and businesses and community systems are developing. Hand in hand with this progress, is the development of corruption. Corrupt practices make life harder for many, with the heaviest burden falling on the people at the lowest levels in the system. Official school fees, for example, are abolished, but have been replaced by unofficial school fees in at least some areas. On the other hand, the Government is actively promoting 'Education for All' and makes an effort to include children with disabilities in the education system. Negotiations are proceeding to develop a Human Resource Management Plan. Capacity building plans are written and were recently officially approved. The Government is making an effort to decentralize power to the provinces and districts. However, all these initiatives to improve the education system still have a long way to go.

\subsubsection{Education in Cambodia}

The turbulent history of the country has left deep and long-lasting scars, from which the education system is still suffering. The Khmer Rouge regime killed almost all people with higher education, after closing down schools and destroying the existing education system. After the fall of the Khmer Rouge regime, the education system had to be rebuilt completely. This process was started during the Vietnamese occupation, continued under sovereign Cambodian power after the liberation from the Vietnamese and is still continuing today.

In 2005, primary school (grades 1-6) enrolment was at 92\%, including 91\% of girls. But this number drops rapidly when the lower secondary levels are reached (grades 7-9): the net enrolment rate is $26.1 \%$ and declines to $9.3 \%$ for upper 
secondary school (grades 10-12)(SCW, 2006). Girls become increasingly underrepresented further up in the education system. Repetition is high and in some cases linked to the inability of parents to settle informal tuition fees.

Dropout rates for the first and second grades are high, at $41 \%$ and $25 \%$ respectively. At the moment, around half a million children in Cambodia do not attend school. They are living mostly in remote and rural areas (SCW, 2006).

The Cambodian Government, together with the help of donors and NGOs, is working hard towards a better education system in accordance with the 'Education for All' Millennium Goal, and their 'Inclusive Education' and 'Child Friendly School' approaches are core elements. The country has signed up to the Dakar Millennium Goal for education and has made the commitment to guarantee basic education to all children in Cambodia by the end of 2015 (NEFAC, 2003).

The Cambodian 'teacher pool' is characterized by its great differences in educational levels. In the early stages after the Khmer Rouge era, it was impossible to assign qualified and well-educated teachers to schools, simply because there were not many left. Instead, the Government appointed as teachers those people who were able to read and write. Many of these early teachers are still working without any formal training. On the other hand, teacher training is now institutionalized and scholarships are provided to help poor and ethnic minority students to become teachers.

\subsubsection{The Ministry of Education, Youth and Sports}

The Ministry of Education, Youth and Sports (MoEYS) is one of the bigger Ministries in Cambodia. It is divided into four Directorates, an Inspectorate and a Secretariat. The Minister heads the Ministry and is supported by a number of Secretaries and Under-secretaries of State (MoEYS, 2005b). This research is conducted in and at the request of the DGE. This Directorate is responsible for basic education throughout the country. Vocational training, however, is under the responsibility of the Ministry of Labour.

The Directorate General of Education consists of the DGE office, chaired by His Excellency the Director General, and two Deputy Directors. The office has a staff of five people. At the time of research, the DGE was responsible for the 
coordination of the work of the seven Departments of Basic Education: the Departments of Early Childhood Education, Primary Education, General Secondary Education, Teacher Training, Pedagogical Research, Non-Formal Education and School Health.

At provincial level, there is a Provincial Office of Education (POE), which controls the District Offices of Education (DOE). The DOEs are responsible for Primary Education and Early Childhood Education. All other Departments are represented at POE level only (MoEYS, 2005a).

As teachers were assigned based on their educational levels in the early years after the Khmer Rouge regime, the Ministry staff was selected on the same principles. Without proper funding or sufficient background in education, and with a lack of support from their political leaders, the staff tried its best to develop an operative education system offering education for students of all levels and grades. With the improvements in the education system, more educated students finished training and were appointed to the Ministry of Education. Staff working in the Ministry of Education has the same widely variable level of education and background as the staff in schools. Key responsibilities of Ministry staff are to develop education policy, provide training services to provincial, district and school staff, collect data and monitor and evaluate the education delivered throughout the country.

\subsubsection{Implications for the study}

The sections above describe the many difficulties the Cambodian Government faces in strengthening civil services. The Cambodian context is highly complex. The country is extremely poor, ranking 136 out of 179 on the 2008 UNDP Human Development Index (United Nations Development Programme [UNDP], 2008). Government staff is underpaid and in need of capacity building, basic materials are lacking and support for change is usually missing. The influence of culture is intricate and affected by the recent tumultuous past. Attempting to understand the context and why things are as they are is difficult. There are usually many reasons, historical and cultural, why decisions and programs are not executed as agreed. These reasons are poorly communicated or not communicated at all. Working collaboratively is a difficult task because it is rare for people to express their personal feelings or ideas. 
Research in Cambodia is further complicated by the fact that the available academic literature on local professional development specifically, and on Cambodia in general, is extremely limited. A consequence of the aftermath of the war is that the small, recently emerged Cambodian academic society has not yet been able to establish a knowledge base of the Cambodian context. Ayres (2000) concludes that publications about Cambodia's recent past and present are minimal. What the previous academic society had established was destroyed during the war and what survived is now outdated. Documents that are available mainly provide a Western perspective of the Cambodian context and are usually written in the form of 'ego-documents' (see, for example, O'Leary, 2007). These documents provide a reflection of personal experiences, which are not necessarily objective and are not grounded in robust research. Almost all of these documents are written by foreigners. As Thion (1993, in Ayres, 2000, p. 5) found: 'Explaining Cambodia is typically a foreigner's business'. This situation does not seem to have changed. The academic basis for professional development through training in developing countries in general is also limited. In particular, the training component is rarely described. The available literature on capacity building, professional development, human resource management and adult learning is exclusively Western.

The research question will be presented next, followed by a brief introduction to the research approach. It is hoped that this research will contribute to a better understanding of professional learning in this fascinating country in Southeast Asia and, on a more global scale, how professional development activities in similar contexts could be made more effective.

\subsection{AIM OF THE STUDY AND RESEARCH QUESTION}

There is a need to improve professional development activities within the basic education sector of MoEYS. This research tries to identify characteristics of more effective PDAs and, during that process, acquire a better understanding of the context. The study provides an analysis of why professional development activities designed and delivered by the Ministry of Education in Cambodia are not successful and what an effective PDA scenario might look like if it is to promote utilisation of acquired knowledge and skills by Ministry staff in their

professional work. The development of an effective scenario for professional 
development activities is the main body of the research. The focus is on how the application of acquired competencies by Ministry staff could be strengthened. The main research question for this study is formulated as follows:

What characteristics should an effective professional development scenario have, when its aim is to strengthen the training facilitation skills of Ministry of Education staff in Cambodia?

The research question can be divided into three sub-questions:

1. Which principles for the design of such a scenario can be derived from a context analysis and a literature review?

2. What are the effects of such a scenario on the acquisition and utilisation of knowledge, skills and attitudes by the staff members?

3. To what extent is the learning of the staff members influenced by (organisational) culture?

\subsection{OVERVIEW OF FOLLOWING CHAPTERS}

The next chapter describes the research approach, justifying the choice for educational design research. Chapter Three provides the context analysis, exploring Cambodian history and culture, before describing recent and desired professional development within MoEYS. Chapter Four examines available research on various relevant aspects of the research question: Human Resource Management, professional development, curriculum development, adult learning, and culturally relevant learning. Based on the context analysis and the exploration of literature, a set of design principles is developed in order to shape the scenario described in this dissertation. The scenario evolved gradually over time and is described as the 'enacted scenario' in Chapter Five. The intervention is evaluated in Chapter Six. The final chapter discusses the outcomes of this research, with theoretical and methodological reflections, and provides recommendations for policy, practice and research that are relevant for this and similar settings. 


\section{CHAPTER 2 \\ Research approach and design}

\subsection{RESEARCH APPROACH}

\subsubsection{Educational design research}

This research is performed as educational design research. Educational design research involves the creation of interventions or scenarios that aim at improving educational processes, developing effective products, or both, and is appropriate for addressing complex design problems in uncertain and dynamic contexts, such as the Cambodian one. The Cambodian government committed itself to extensive educational reforms, but Ministry of Education, Youth and Sports (MoEYS) staff lacks the professional capacity to implement these reforms. Furthermore, the influence of (recent) history, cultural habits and social hierarchies in Cambodia is important, but scarcely studied. The limited (academic) knowledge base on professional development in Cambodia and the cultural aspects influencing it does not provide much direction on how to improve learning trajectories for Ministry staff.

Educational design research can be characterised as: a) interventionist: a particular intervention or scenario is designed and implemented in an actual 'real-world' setting; b) iterative: a cyclic process of design, evaluation and revision is followed; c) process-oriented: aimed at understanding the process and improving the intervention; d) utility-oriented: aimed at being as practical as possible; and e) theory-oriented: based on existing theory and aiming at contributing to theory building of poorly understood contexts (van den Akker, 1999). One of the applications of educational design research is: 'shaping an innovative intervention and developing a theory that underpins the intervention' (Kelly, 2006, p. 108). 
Educational design research aims at finding solutions for problems in contexts, by combining a systematic design and evaluation of validated solutions with extending the knowledge base through empirically tested and theoretically grounded design principles (van den Akker, 1999). Design research adopts an interactive and cyclic process, in order to arrive at balanced outcomes that satisfy the formulated needs and are appropriate for the context. Van den Akker (1999) describes this process of iteration as successive approximation.

The socio-constructivist and collaborative interaction that is essential to arrive at workable solutions could also be seen as a professional development scenario in itself (see Guskey, 2000). It is this latter notion that is investigated further as a way of professional development of government staff in the Ministry of Education in Cambodia. At the same time, involvement of the users in the design process raises their commitment to, and ownership of, the solutions found.

This form of research could also be categorised as exploratory and developmental. It is exploratory because it is often conducted in contexts of which little validated knowledge exists and investigation is necessary in order to develop contextual understanding. It is developmental, as the aim is to develop successive prototypes of products or processes in a collaborative and cyclic manner and to identify validated design principles that are specific for that context (van den Akker, 1999). Data are obtained through dialogue with and observation of the people involved. These qualitative data are essential for understanding the historical, cultural and social backgrounds of the context, and contribute to the meaning-making process about that particular context (Kelly, 2006). A widely generalisable theory is not the goal of educational design research. Instead, by describing characteristics of the context in which the scenario is executed, hypothetical generalisability is pursued. It is important to note that the completed research described in this dissertation is not action research. Action research involves participants who investigate their own behaviour and roles in education processes. Although participants were actively involved in shaping the scenario, it was the researcher who conducted the research.

\subsubsection{Validity and reliability of educational design research}

Validity and reliability deserve special attention when conducting educational design research. Reliability was fostered by the fact that the researcher used the same instruments during all interviews and observations. Data triangulation - in 
the sense of using multiple measures to investigate events (Yin, 2003) - and the use of three established models for evaluating the scenario both aimed at enhancing validity. In this research, interviews, informal dialogues, evaluation forms, and expert feedback were used to triangulate findings. The three evaluation models (described later in this chapter) informed the construction of the research tools as well as the data analysis.

\subsubsection{Quality criteria for products and scenarios}

The quality of a scenario that is developed during educational design research is determined by its relevance, consistency, practicality and effectiveness (Nieveen, 2009; van den Akker, 1999). The relevance criterion helps to ensure that a scenario is based on an actual need and 'state-of-the-art' knowledge. Consistency aims at developing a 'logical' design, while the practicality criterion indicates the extent to which the scenario is of practical use to the settings it is designed for. The effectiveness criterion assesses the extent to which usage of the scenario results in the desired outcomes.

\subsection{RESEARCH DESIGN}

\subsubsection{The research design phases}

The research applied in this dissertation consisted of three phases: 1) a preparatory phase; 2) a design and implementation phase, and 3) an evaluation phase. The phases are depicted in figure 2.1.

During the preparatory phase the context, existing knowledge base, and needs and wishes of MoEYS were analysed. The outcomes of the preparatory phase led to a set of design principles and an initial scenario design. The scenario was further developed during the rest of the design and implementation phase. The evaluation phase looked back on the scenario development process and its outcomes. Although the stages are presented in an orderly sequence, in reality they sometimes overlapped. The next section will sketch the three phases in more detail. 


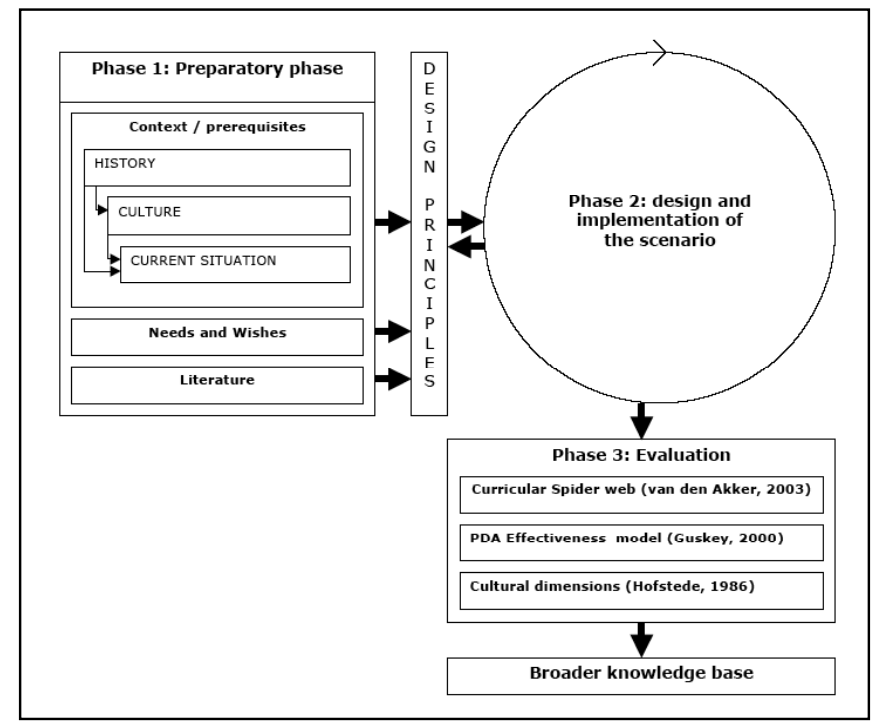

Figure 2.1 Overview of the three phases of the research design

\subsubsection{The preparatory phase}

The preparatory phase started in February 2006 and lasted thirteen months. It started with a two-month Khmer language course for the researcher, additional intensive language lessons and a subsequent introductory period at MoEYS, during which colleagues were introduced and the tasks to be completed were discussed. The researcher became part of the Capacity Building Coordination Group (CBCG), in which the seven Departments of the Directorate General of Education (DGE) were represented.

During this phase, a thorough context analysis, an analysis of needs and wishes within the Ministry, and a literature analysis were conducted. The context and the needs and wishes within the Ministry were analysed by: 1) observations of trainings and workshops; 2) analysis of documents (e.g.: needs analyses, project proposals and evaluations on professional development within MoEYS); and 3) interviews with Department Directors.

The literature analysis explored, on the one hand, Cambodia's history and culture related to learning and education and, on the other, contemporary theories on human resource development, professional development, curriculum development, adult learning and culturally relevant learning. Most of the academic literature was found in libraries in Thailand, Malaysia and the 
Netherlands, and on the web. It proved to be impossible to identify literature that connected professional development and learning to the Cambodian context, emphasizing the importance of the research presented in this thesis and its contribution to the existing knowledge base. The findings resulted in the formulation of a set of design principles, which then formed the basis of an initial scenario design that was developed in the next phase. The outcomes of the context analysis and the way history and culture influence learning in Cambodia are described in Chapter Three. Chapter Four focuses on the academic backgrounds of professional development and adult learning theories.

In order to be able to develop a thorough understanding of the local context, the local practice of professional development and the perceptions of stakeholders needed to be investigated. Important questions at this stage were: How is professional development organised and conducted in Cambodia? What knowledge and skills do people possess and how do they use their experience to make judgement calls? What are the strengths and weaknesses of professional development endeavours? How do people, both trainers and trainees, see the way they build capacity and what would they like to change in their approaches? What do people expect from the researcher and how do they see their own contribution to change?

An initial meeting with the Deputy Director of the DGE provided the Ministerial point of view. Six interviews were arranged (Annexe 1) and conducted with Directors or Deputy Directors of the Departments of the DGE in order to collect the Departmental opinions. Some of the (Deputy) Directors were not able to speak English. Therefore, these interviews were conducted in Khmer. A semi-structured interview method was adopted, which made it possible to pursue interesting leads but still retain a basic structure in the interview (Annexe 2). Informal conversations also provided interesting data that were recorded in the research log.

Six observations of different professional development endeavours were carried out on different dates, in order to develop a picture of the ways in which training and workshops were provided.

MoEYS' major aspiration was to improve the quality of professional development activities provided by the Ministry. The Ministry asked the researcher to develop an intervention. The aims of the intervention were: a) to develop a set of Training 
Guidelines universally applicable in the Departments; b) to develop an evaluation methodology for professional development activities (PDAs); c) to build the capacity of the staff on training and facilitation skills; and d) to arrive at a more effective scenario to strengthen professional development of its staff.

The primary outcome for the research was to obtain insight into what a more effective scenario for professional development of Government staff in MoEYS could look like, and how it had been influenced by (organisational) culture. The other aims formed the content on which to focus during the scenario.

\subsubsection{The design and implementation phase}

The second phase started with the initial scenario design for effective learning by MoEYS staff, based on the design principles delivered at the end of the preparatory phase (presented in the next two chapters) and the needs and wishes of MoEYS.

The initial scenario design included a working group that would hold regular meetings, develop a four-day training course for MoEYS staff, and the delivery of the four-day training course itself. During the design and implementation phase, the working group further developed the initial scenario design into the enacted scenario by adopting an iterative process in which design and implementation informed one another.

The character of educational design research was implemented at two levels. The first level encompassed the whole scenario from the first meeting of the working group, until the final day of the four-day training. As the scenario evolved over time, each event during the scenario informed the next step. For example, the experiences of one working group meeting shaped the way the next meeting was organised. This 'trajectory' was completed once during this research, but provides several recommendations for the conduct of future scenarios.

The second level involved a number of smaller iterative processes referred to as 'micro-cycles', which informed the gradual improvement in knowledge and skills of the group members and the development of materials produced during subsequent meetings of the scenario. Training methodologies and tools like checklists and evaluation forms were developed, tested, discussed and adapted. 
In November 2006, both the Deputy Director and CBCG of the DGE approved the initial design (Annexe 3). The project was formalised in the MoEYS-DGE Capacity Building Mid-Term Plan 2008-2010 (Berkvens, 2007a) and approved by UNICEF Cambodia. The working group was provided with a mandate to develop a Training Guideline, a four-day training, evaluation tools, and a scenario for more effective professional development in order to improve the training and facilitation skills of the working group members. The Departments and the DGE were invited to delegate one staff to the working group, leading to a community of nine learners.

At the start of the scenario, the researcher interviewed all working group members in order to investigate their education levels, previous experience with training and facilitation, and expectations of the project. A semi-structured interview approach was used (Annexe 4). Based on the outcomes, the start level of the group members, and possible subjects that needed to be addressed during the working group meetings were determined (Annexe 5).

All working group meetings were observed. Informal conversations and observations of PDAs that were organised by group members outside the scenario delivered further data on the application of acquired knowledge and skills by the group members. Final interviews with the working group members were conducted six months after the final working group meeting and before the start of the preparatory meetings of the Training Guideline training, in order to assess long-term retention and application of knowledge and skills (Annexe 6).

The preparatory meetings were planned over a two-month period, in order to allow the group members enough time to collaboratively prepare the four-day Training Guideline training. The researcher observed both the preparatory meetings and the training, and held informal discussions with the group members. The gathered data provided insight on how acquired knowledge and skills were implemented in reality, i.e. how the group members used the acquired knowledge and skills as facilitators of the Training Guideline training.

\subsubsection{The evaluation phase}

The 'curricular spider web' (van den Akker, 2003), the 'five critical levels of professional development model' (Guskey, 2000), and the '5-D model of cultural 
differences' (Hofstede, 1986) consisting of five cultural dimensions, were used to evaluate the relevance, consistency, practicality and effectiveness of the enacted scenario. The relevance of the enacted scenario was determined by the extent to which the scenario met the needs and wishes of the Ministry on the one hand, and how well it was grounded in current academic knowledge on the other. The curricular spider web was used to assess the relevance and consistency of the enacted scenario. The PDA effectiveness model and the 5-D model of cultural differences were used to measure the practicality and effectiveness of the scenario.

The evaluation phase was formed by observations of all working group meetings, the preparatory meetings and the Training Guideline training course. The outcomes of the final interviews with the group members, informal conversations and the evaluation forms that were filled out at the end of the last working group meeting further complemented the data on which the evaluation was based (Annexe 10). A coding list was established for the analysis of the data (Annexe 7).

\section{The five critical levels of professional development evaluation model}

The five critical levels of professional development evaluation (Guskey, 2000) were used to assess the practicality and effectiveness of the scenario. Using the model, the perceived (by group members) and observed (by the researcher) professional development of the working group members after participating in the working group were investigated. Table 2.1 shows the model consisting of five levels. The first level of 'participants' reactions' measures how people experienced the learning endeavour. The next level assesses what the participants perceive themselves to have learned. The third level investigates how their organization supported learning, followed by the fourth level that examines the extent to which the learners used the newly acquired knowledge and skills. The fifth level assesses how the professional development endeavour affected secondary beneficiaries' (i.e. students') learning outcomes. The second and third columns were slightly adapted, in order to gather information relevant for this research. 
Table 2.1 Guskey's five critical levels of professional development evaluation model (adapted) (Guskey, 2000, pp. 79-81)

\begin{tabular}{|c|c|c|c|}
\hline Evaluation level & $\begin{array}{l}\text { Questions } \\
\text { addressed }\end{array}$ & $\begin{array}{l}\text { Information } \\
\text { gathering }\end{array}$ & $\begin{array}{l}\text { Measured or } \\
\text { assessed }\end{array}$ \\
\hline $\begin{array}{l}\text { 1. Participants' } \\
\text { reactions }\end{array}$ & $\begin{array}{l}\text { Did they like it? } \\
\text { Was their time well } \\
\text { spent? Did the } \\
\text { material make } \\
\text { sense? Will it be } \\
\text { useful? Was the } \\
\text { room good? }\end{array}$ & $\begin{array}{l}\text { Evaluation forms, } \\
\text { evaluation activi- } \\
\text { ties, interviews, } \\
\text { informal conver- } \\
\text { sations }\end{array}$ & $\begin{array}{l}\text { Initial satisfaction } \\
\text { with the experience }\end{array}$ \\
\hline $\begin{array}{l}\text { 2. Participants' } \\
\text { learning }\end{array}$ & $\begin{array}{l}\text { Did participants } \\
\text { acquire the intended } \\
\text { knowledge and } \\
\text { skills? }\end{array}$ & $\begin{array}{l}\text { Participant reflec- } \\
\text { tions, observations, } \\
\text { informal conver- } \\
\text { sations }\end{array}$ & $\begin{array}{l}\text { New knowledge } \\
\text { and skills of } \\
\text { participants }\end{array}$ \\
\hline $\begin{array}{l}\text { 3. Organization } \\
\text { support and } \\
\text { change }\end{array}$ & $\begin{array}{l}\text { What was the } \\
\text { impact on the } \\
\text { organization? Did it } \\
\text { affect procedures? } \\
\text { Was implementa- } \\
\text { tion advocated, } \\
\text { supported and } \\
\text { facilitated? Were } \\
\text { there sufficient } \\
\text { resources? }\end{array}$ & $\begin{array}{l}\text { Minutes, interviews, } \\
\text { observations, } \\
\text { informal conver- } \\
\text { sations }\end{array}$ & $\begin{array}{l}\text { The organization's } \\
\text { advocacy, support, } \\
\text { accommodation, } \\
\text { facilitation and } \\
\text { recognition }\end{array}$ \\
\hline $\begin{array}{l}\text { 4. Participants' use } \\
\text { of new } \\
\text { knowledge and } \\
\text { skills }\end{array}$ & $\begin{array}{l}\text { Did participants } \\
\text { effectively apply the } \\
\text { new knowledge and } \\
\text { skills? }\end{array}$ & $\begin{array}{l}\text { Participant reflec- } \\
\text { tions, interviews, } \\
\text { observations, } \\
\text { informal conver- } \\
\text { sations }\end{array}$ & $\begin{array}{l}\text { (Relative) degree } \\
\text { and quality of } \\
\text { implementation }\end{array}$ \\
\hline $\begin{array}{l}\text { 5. Secondary } \\
\text { beneficiaries (i.e. } \\
\text { student) } \\
\text { learning } \\
\text { outcomes }\end{array}$ & $\begin{array}{l}\text { What was the } \\
\text { impact on students, } \\
\text { did it affect student } \\
\text { performance and } \\
\text { achievement? Did it } \\
\text { influence students' } \\
\text { physical or emotio- } \\
\text { nal well-being? }\end{array}$ & $\begin{array}{l}\text { Observations, inter- } \\
\text { views, informal } \\
\text { conversations, } \\
\text { evaluation forms } \\
\text { for participants }\end{array}$ & $\begin{array}{l}\text { Student learning } \\
\text { outcomes (affective, } \\
\text { psychomotor, } \\
\text { cognitive) }\end{array}$ \\
\hline
\end{tabular}




\section{Van den Akker's curricular spider web}

The scenario could be seen as a curriculum that was established through 'learning by designing'. The curricular spider web model of van den Akker (2003) was used to assess the relevance and curricular consistency of the scenario, rather than for developing the scenario. Figure 2.2 depicts the 'curricular spider web'. The rationale is the body of the curriculum and is based at the core of the web, surrounded by the different components: aims and objectives, content, learning activities, teacher role, materials and resources, grouping, location, time and assessment. How curricula can be used to support learning will be discussed in more detail in Chapter Four.

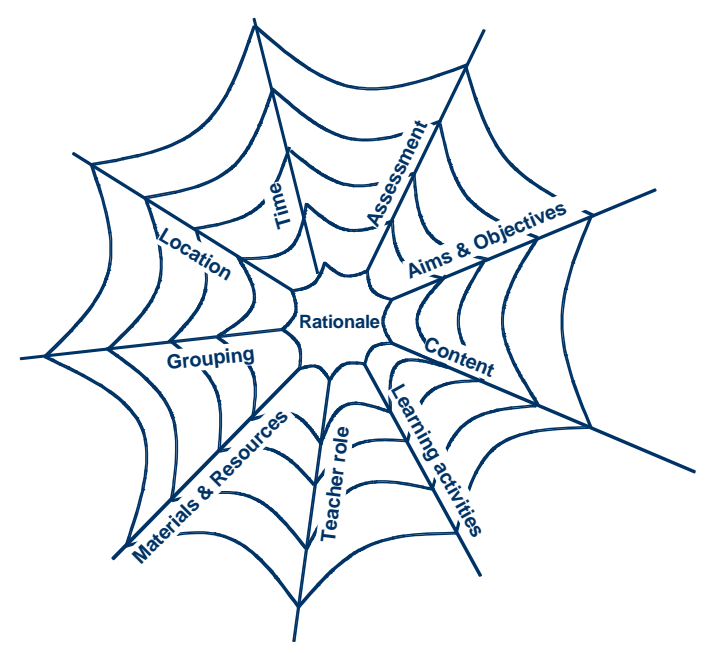

Figure 2.2 Curricular spider web (van den Akker, 2003)

\section{The 5-D model of cultural differences}

Hofstede (1986) describes five cultural dimensions (hence, the 5-D model) that are found in every culture, but in different ratios. He distinguishes individualism versus collectivism, power distance, uncertainty avoidance, masculinity versus femininity and long-term versus short-term orientations. The dimensions are discussed in more detail in the next chapter, in which these five dimensions are used to investigate the influence of Cambodian culture on professional development.

\subsubsection{Data collection}

At the start of the scenario, all members of the community of learners were interviewed. The interviews were semi-structured. All working group meetings, 
preparatory meetings and sessions of the Training Guideline training were observed. At the final meeting of the working group, the members were asked to fill out an evaluation form. Throughout the entire scenario, numerous informal discussions were held, and notes kept.

Consent and privacy

At the start of the project and at the beginning of each interview, it was made clear to the participants that the researcher would only use the interview data for the purpose of this particular research. It was explained that statements would only be published without a reference to specific persons. All interviewees agreed to these safeguards, gave their consent and cooperated freely.

\section{Taping interviews and observations}

Unfortunately, reliable tape recorders only became available during the second half of the research period. The earlier interviews were conducted by taking notes and were immediately transcribed after each interview. Later, when a tape recorder was used, permission to record the interviews was always asked from the interviewees. Interviewees were invited to read the transcript, but none did.

The choice of not videotaping the meetings, training courses and workshops was deliberate. It was feared that videotaping endeavours might be too threatening for some people. The fact that traits like 'fear' and 'shyness' are abundantly present amongst Cambodians led to the decision that creating a safe learning environment was more important than more complete data gathering. Photographic images were taken instead, but only when it was not too distracting to do so.

\subsection{MiNiMIZING BIASES}

Minimizing biases and errors makes research more reliable (Yin, 2003). Threats of biases identified in this research described in this dissertation are discussed below.

\subsubsection{Role bias}

The researcher had multiple roles during the group meetings, being a designer, facilitator and researcher. Extra attention was given to prevent role bias. One way 
was to thoughtfully and carefully investigate the data collected and reflect on processes 'for action', 'in action' and 'on action'. A second way for the researcher to prevent role bias was to decide in advance what roles the researcher would adopt during meetings, for example the choice to be mainly an observer during the four-day Training Guideline training. Such decisions on role separation were not always possible nor desirable, as the combination of different roles also contributed to the gaining of insights.

\subsubsection{Insider/outsider bias}

The researcher belonged sometimes to the insider group and, at other times, to the outsider group. This is reflected upon in more detail in Chapter Seven. The important point here is that the researcher had to understand - and check - his position as perceived either by the group members or by himself, and the consequences of that.

Language was also an important issue. In most cases interviews were conducted in English, as it was easier to go into more detail than when the interviews were done in Khmer. On the other hand, sometimes Khmer was used when things were unclear to either the interviewer or the interviewee. The researcher himself conducted these interviews in Khmer, avoiding the use of a translator as translations in Cambodia are rarely precise. During translation, information is often added or omitted. Translators tend to turn open-ended questions into dichotomous ones and neutral questions into negatives. This is not deliberate, but a consequence of the Khmer language structure.

\subsubsection{Cultural bias}

The following two chapters show how much the development agenda is influenced by Western beliefs and expectations. The chapters also give examples of how foreign advisers were sometimes unaware of their Western biases. That is probably the case with this research as well. Definitions can have different connotations in other cultures, and it takes a stay of an extended period in a culture to understand such differences. Furthermore, cultural habits and characteristics might also be different. For instance, 'shyness' is a recurring cultural characteristic of Cambodians that can come from feeling embarrassed to disturb somebody, genuine politeness, or a taboo on a certain subject of event, 
which could easily be misinterpreted when cultural and contextual understanding is ill developed. The interpretation of non-verbal communication and the importance of observing what is not happening are other issues described by Patton (1990), although he did not attribute them to cultural differences. Being sensitive to different cultural behaviour is essential. In Cambodia, for example, people tend to conceal their emotions, making it difficult to read them. But it does not mean that emotions are not there, just that one needs to learn how to recognize them, and check if interpretations reflect the actual emotional feelings.

\subsubsection{Response bias}

Response bias - providing desired answers to please the researcher - can almost be seen as a specific form of cultural bias within the Cambodian context. It is an issue for every piece of research depending on interviews anywhere, but in Cambodia it is amplified by the cultural disposition to please other people on one hand and the fear of being seen as stupid on the other. Double-checking answers and probing for more background information were both used to minimize the risk of response bias.

\subsubsection{Selection bias}

The selection of the working group members was outside the control of the researcher. The Department Directors selected the group members based on their prior experience with professional development and English language skills.

\subsection{LIMITATIONS OF THE STUDY}

\subsubsection{Small sample size and absenteeism}

Absenteeism at work meetings is a recurrent issue in Cambodia and was also common during the meetings of the working group. The most common reasons were: 1) lack of planning skills; 2) high workload;3) hierarchical demands; and 4) financial benefits.

Academic skills of Ministry staff are poor, including the skills needed for planning. Workload is usually high for the most capable staff, making it 
impossible to meet all the demands placed on them. Higher-ranked officials often call for sudden meetings, or decide in favour of other priorities, demanding that staff attend those meetings. Some projects provide financial benefits for staff members who participate. It must be noted, however, that group members on multiple occasions said they joined because it would strengthen their capacity, which was more important to them than receiving a 'per diem' (remuneration) payment.

Invitations were distributed a week before each meeting, and members were reminded of the meeting the day before. Both strategies were undertaken in order to optimize participation. Reports, in the form of printouts, were circulated after each meeting to all members of the working group, regardless of attendance, to keep all members informed of the progress made.

\subsubsection{Limited generalisability}

The study was conducted within the DGE in the MoEYS. Although observations of PDAs have been conducted inside the broader Ministry and in other Ministries as well, the scenario has only been developed with the DGE and its Departments' staff. Therefore, generalizations of the outcomes of the scenario could not easily be made. The principles that formed and were identified by the scenario, however, are similar for other Directorates inside MoEYS and the broader Government. The method of hypothetical generalisation has been discussed in the first section of this chapter and is used in Chapter Seven to extend the generalisability of this research. The more thorough the 'thick description' of the research context, approach and outcomes is, the more generalisable the findings of this research are for other, similar contexts. 


\section{CHAPTER 3 \\ Context analysis}

\subsection{INTRODUCTION}

This chapter aims to identify context-specific design principles in order to develop an effective scenario for professional development in the Ministry of Education, Youth and Sports (MoEYS) in Cambodia through an analysis of the context. Understanding the current situation of and need for continuing capacity development starts with comprehension of the history of Cambodia and its impact on society, the education system and civil services. It is far too easy to perceive the Khmer Rouge regime as the source of all problems the country currently has, as many scholars and writers have tended to do. Guidebooks, documents and presentations start their introduction by referring to the Khmer Rouge period. Only a few authors acknowledge that many of Cambodia's problems are much older than that (see Ayres, 2000; Verkoren, 2005). However, while acknowledging the difficulties of the past is important because it helps to understand current difficulties, it is also important to recognize the achievements of the Cambodian Government over the last fifteen years: schools have been constructed, health care is provided, infrastructure built and the capacity of the civil service strengthened. A broader description of Cambodia's history is outside the scope of the research presented in this dissertation. Chandler (2007) provides a thorough analysis of the country's history.

This chapter commences with the place of education throughout Cambodian history. It will show how education has followed Cambodian political history and how it was and remains influenced by the culture. The social hierarchy system of Cambodian society with strong ancient notions of power on one side, and calls for equality (initially introduced by the French) on the other, remained 
strong throughout history and was at the basis of the 1970's conflict. Buddhism, the religion of the majority, is at the centre of the ongoing conflict between these modernizing and more traditional forces.

After independence from the French in 1953, King Sihanouk, followed by Lon $\mathrm{Nol}$ and later the Khmer Rouge, were unable to refrain from absolute power. Current leaders demonstrate some of the same features as their predecessors did, in the sense that they expect to have total control over society without allowing constructive criticism that could help to improve the state. Intellectuals and students were the much-needed supporters of the Sihanouk and Lon Nol regimes. King Sihanouk especially expended great efforts on education.

Cambodian education will be discussed in four periods: 1) education before and during the French protectorate and early independence, 2) education during the Khmer Rouge era, 3) education during the Vietnamese occupation and after, and 4) education based on Cambodia's recent ambition to become part of the globalised world.

As Hofstede's (1986) 5-D model for cultural differences illustrates, culture has impact on the way people learn. Cambodian culture is complex, partly because it was disturbed by the happenings of the recent past. It also makes it difficult to generalize the nation's psyche with that of its neighbours. Thus, a more thorough exploration of the Cambodian culture to understand its influence on professional development in Cambodia is needed. The 5-D model will be used as a framework for analysis, with variations that emerge from this further exploration, converging into a succinct description of Cambodian culture and implications for professional development in Cambodia.

The third issue covered in this chapter is the important - but often dubious - role of the international (aid) community in professional development in Cambodia. The chapter explores the way aid organisations dominate the aid agenda and identifies alternatives as to how they should act in order to support professional development more effectively. 


\subsection{THE HISTORY OF CAMBODIAN EDUCATION}

\subsubsection{Education before and during the French protectorate and early independence}

Cambodian history until the 1970's consists roughly of the pre-Angkorian period, the Angkor period, followed by a long period of peasantry, the French protectorate and the sovereign monarchy of King Sihanouk. During the preAngkorian and Angkorian period, Indian migrants influenced large parts of Southeast Asia, including what is now known as Cambodia. Their influence on the Khmer social system is characterised by the importance of reciprocal relationships and dependencies, leading to a complex social hierarchy. Kings were persons of almost mythical proportions, protecting society. The Angkor Empire had its most powerful period halfway through the twelfth century. The temples of Angkor symbolise the traditional Cambodian concepts of power, embodying absolutism and hierarchy (Ayres, 2000).

Buddhism has always been a strong factor in Khmer society not only as a religion, but also as a political power and educational channel for spreading norms and values. The doctrine of Buddha, with its pillars of harmony, balance and conformity, believes that humans are not perfect and essentially helpless. Buddhism did not question the necessity of authority, like a King, and a strong hierarchical social order. Traditional education, with principles on how to be a good individual and a person of good conduct, was and is still spread by monks and through pagodas. Many Cambodians were illiterate, explaining why oral stories and anecdotes were used to spread the Buddhist principles of reciprocal relationships, dependencies and hierarchy, alongside prescriptions of harmony, balance, regularity and conformity (Ayres, 2000).

When the French forced a protectorate upon Cambodia in 1863, their mission aimed to assimilate the Khmer (ethnic Cambodians) into a French-style system of governance. Much more forcefully than the British in their colonies, the French strove for elimination of parochial habits and promoted a culturally undifferentiated society. Uniformity, egalitarianism and European rationality were spread. Centralized administration and precision in legal and constitutional matters were implemented in civil society. Although executed half-heartedly, the French felt an obligation to improve the social, cultural and material status of the 
Khmer. They did so with a complete lack of understanding of Cambodia and its people. Policy and methodologies were prescribed by Paris and were egalitarian in nature, a concept that was far from Cambodian reality (Ayres, 2000).

The French did not really make an effort towards education for the masses in Cambodia. Figures dating from around 1900 show that of the hundred pupils in French language schools, only eight were Cambodian (Humbert-Hesse, 1923). Under the French protectorate, Khmers were compelled to study in neighbouring countries or in France, but in 1944 there was still only one Khmer engineer in the whole country (Tully, 2002). It was for financial and managerial reasons that the French did not build a separate education system, but relied on existing temple schools for rolling out their education agenda. The initial plan was to reform education that was provided by the temple schools, but this never happened. Temple schools were characterised by reinforcing traditional systems and beliefs ensuring social cohesion and maintenance of traditional values, leading to a clash between traditional values and beliefs of the monk-teachers and content of the French-imposed curriculum (Ayres, 2000; Tully, 2002). Commitment by the monks was low and student learning limited. Monks could leave the pagoda as they liked at any time during lessons. Tully (2002) notes that peasants saw little value in education and were reluctant to send their children to school, as they could also contribute to the family economy by working in the fields. The transformation the French had in mind was never installed in the countryside. Only the children of the elite in the cities received a higher form of education, although the French tried to create a gateway for some underprivileged into the administrative and elite corps. As a result, at the end of their rule in 1953, the French had been unable to spread their egalitarian philosophy as they had in Vietnam and their African territories. They did, however, help to reinforce the power of the Cambodian monarchy and raised the profile of King Sihanouk.

The era of King Sihanouk's rule over Cambodia, from the 1950's to the 1960's, in the beginning under French protection, still has a kind of mythical connotation for many Cambodians. Sihanouk promoted an ideology he called 'Buddhist Socialism' to move the country forward. His philosophy was socialist, as it stated that people should be treated equally, with empathy and goodness. On the other hand, it was not socialist at all. Sihanouk was a ruler with totalitarian features, who also claimed that people should be allowed to enjoy the benefits of their efforts. Charity was promoted as a way to share wealth and take care of the poor. This ideology 
enabled Sihanouk to retain total control while promoting economic development and following Buddhist teachings. Ayres (2000) shows that Cambodians regularly refer to the period of Sihanouk's rule as Cambodia's 'Golden Age', but he negates this general belief by describing it as a regime characterised by political repression, state-sanctioned violence, factionalism, corruption, incompetence, and absolute contempt by the powerful towards those ruled by them. Fröberg Idling (2009) has a more positive interpretation, showing that although the regime was corrupt, it also worked towards prosperity for the country.

Education under Sihanouk spread quickly. In order to develop the country, the King raised the profile of education by making attendance compulsory for the young and motivating parents to send their older children to school as well. People not only saw education as a means towards personal development, but also as a way to enhance the country's development. Some argue that this was Sihanouk's way to legitimize his Government and reinforce nation building. Many Cambodians saw education as a social investment and a means of social mobility. They embraced the hope for a better future up to the point where educational demand outweighed supply, and budgets where too limited to serve all (Ayres, 2000).

Table 3.1, 'Number of students in Cambodia per kind, 1930-1960', shows numbers of enrolled students over a thirty year period. The figures show that under King Sihanouk, education for the masses became considerable (Tully, 2002). The asterisk at year 1955 indicates the independency in 1953.

Table 3.1 Number of students in Cambodia per kind, 1930-1960 (Tully, 2002)

\begin{tabular}{|lcccc|}
\hline Year & Primary & Secondary & Technical & Higher \\
\hline 1930 & 65,000 & 310 & 400 & 0 \\
\hline 1935 & 56,000 & 370 & 260 & 0 \\
\hline 1940 & 72,000 & 390 & 320 & 0 \\
\hline 1945 & 89,000 & 680 & 280 & 0 \\
\hline 1950 & 183,000 & 1570 & 280 & 240 \\
\hline $1955^{*}$ & 286,000 & 3810 & 330 & 350 \\
\hline 1960 & 542,000 & 16,840 & 980 & 1430 \\
\hline
\end{tabular}

Note: ${ }^{*}=1953$, year of independence.

These numbers are even more significant, given that the Cambodian population in 1953 was almost five million. In 1960, enrolled students constituted more than 
$11 \%$ of the population. It is also important to note that in 1955 not even 4000 students were enrolled in secondary education and only 350 in higher education, while five years later, those numbers had quadrupled.

However, the sudden increase of students after independence was merely a quantitative increase, and was not accompanied by quality improvements of educational standards. The curricula were still French-based and irrelevant for the Cambodian context, girls were underrepresented and instruction was of disputable quality. Although it was obvious that the state could not support such unbridled growth, King Sihanouk tried to make secondary education compulsory and started a process of 'Cambodianisation' of the educational system. This process changed the language of instruction, the structure of courses and school textbooks. The education system spread throughout the country, as for the first time the state showed its presence in the rural areas (Ayres, 2000). Not only were the values of the state spread through this system, it was also a means to anchor King Sihanouk as the state father in a way that shows signs of indoctrination.

Halfway through the 1960's King Sihanouk opened the Royal University. According to Ayres (2000), critics believed that he was trying to gain admiration for being the King developing Cambodia. Students tended to follow 'modern' courses like arts, instead of much more needed vocational and technical professions. Their choices were a consequence of the Government's rhetoric in favour of modernity. It remains unclear how well trained the students were. Verkoren (2005) suffices with saying that the Cambodian education system before 1970 was 'very well-developed' (p. 302) and that it was not only a legacy of the French but also of the expansionism of King Sihanouk. Others, interviewed by Ayres (2000) and Tully (2002), however, claim that teaching methods were poor, funds and resources were lacking and teachers were ignorant (having been poorly educated themselves). The use of education as a channel for indoctrination adds to the question of how academically skilled graduates in the end were, as indoctrination tends to silence a critical attitude (Risse \& Sikkink, 1999).

The expansion of education led to another problem: more than a million students graduated in four years, against 10,000 available jobs within the Government a year. The unemployed graduates found that the Government was unable to solve their problems, when at the same time corruption and nepotism thrived. Despite some level of indoctrination, the young and educated 
started to question the way the system was run and demanded more influence in how the country was run (Ayres, 2000).

Khmer intellectuals, who were educated and had become intrigued by communist values during their studies in France, returned to Cambodia in the time of awakening nationalism and called for more influence for the people. They started to question the nature of the Khmer social system. They promised the Cambodians what was denied to them by the Royal Government: equality and more widespread prosperity. Ayres (2000) claims that it is unlikely that many teachers and students were true communists: what drove them was merely dissatisfaction with the 'decadence and corruption in Phnom Penh' (p. 61). The Government, however, depended on teachers to represent the regime, but it was these servants who were susceptible to the revolutionary ideas. For instance, many of the leaders of the Khmer Rouge, including Pol Pot, were secondary school teachers. The communist ideology stood for a moral, nationalistic, noncorrupt and equal state, the opposite of what people were experiencing in their daily lives. The teachers who were favourable to communist ideas seemed to hold the only probable solution to the problems in the country. With the authority people invested in teachers, they had an immense influence on the political situation at that time.

To illustrate that Cambodians tend to comply with 'higher authority', whether they agree with it or not, Ayres (2000, p. 58) presents a short anecdote. He tells the story of a teacher in a village who was commanded (by a state official) to assemble his students to counteract 'rebels'. The teacher and his students did so. They did not act by conviction, but were only following the orders of people with higher authority than themselves.

After the deposition of King Sihanouk by Lon Nol at the beginning of the seventies the situation in Phnom Penh deteriorated. The elite continued living an extravagant lifestyle, while corruption and harshness made the lives of the poor and thousands of refugees in the city worse and worse. At the same time, the Khmer Rouge fought its way towards Phnom Penh (Ayres, 2000). Some of the communist intellectuals, like Ieng Sary and Pol Pot, turned their conviction into radical actions. Where the French did not understand Khmer culture very well and thus were virtually unable to break traditional power relations and hierarchical systems, the French-educated leaders of the Khmer Rouge were able 
to do exactly that: they abolished the old hierarchical systems and based their system on Maoist ideas. It led to a peasant society that was characterized by force, violence and oppression, instead of a society based on ideas as spread in Western universities where the Cambodian communist leaders were educated at that time.

\subsubsection{Education during the Khmer Rouge era}

But before the horror of the Khmer Rouge became apparent, many Cambodians were relieved when the Khmer Rouge entered the capital on April 17, 1975. The Khmer Rouge was thought to bring hope after a long period of uncertainty in which both national and international political relations had been confusing. However, the next day the people of Phnom Penh and other towns were forced to leave their cities on foot and go to the paddy fields in the countryside, a journey from which many did not return.

The driving force behind the Khmer Rouge was the former Khmer French student Saloth Sar, later known as Pol Pot. Together with Ieng Sari, Khieu Samphan and other 'brothers', while living as young students in France, they saw Marxism as a promising model for equality and emancipation of their hierarchical and conservative home country and wished to install a completely self-reliant and selfsufficient society (Ayres, 2000). Ironically, the leaders of the Khmer Rouge, being intellectuals themselves trained in France, ordered the dismantlement of city life and destruction of the Cambodian intellectual community.

The Khmer Rouge had conquered the country on mostly false sentiment: they had used King Sihanouk to win the support of the peasants, but put him under house arrest after their victory. They were communists and strove for egalitarianism: Buddhism, ownership of property and the monarchy were terminated. Money was declared worthless and family ties were cut. Individualism, seen as a modern thing from the West, was abandoned in favour of collectivism and anonymity.

Compared to the previous administrations, the Khmer Rouge regime shows some similar and some very different features. Almost all Khmer regimes since independence embraced the power of their ancient predecessors as their own: Khmer superiority and power were seen as unsurpassable. The three regimes, of King Sihanouk, Lon Nol and the Khmer Rouge were conducted with the 
conviction that they were completely legitimate in that sense. Opposition and criticism were eliminated by force. But where King Sihanouk and Lon Nol presented themselves as almost half-divine, Saloth Sar was almost invisible and part of 'Angkar' or 'the organisation', as the system of the Khmer Rouge was called at that time. The Khmer Rouge despised the corruption, decadence, nepotism, exploitation of the poor, and unbridled capitalism of the elite that had thrived under the former regimes.

The Khmer Rouge regime was horrific. Many people died during the marches to the working fields, or from the unbearable harshness of life in the paddies with their long working hours, scarcity of food and lack of medical care. Possible opponents of the regime and intellectuals were tortured and killed. In the concluding years of the regime, even the 'party's internal enemies' met the same fate, through a morbid fear of loss of control. Many of the high-ranking and more intellectual cadres were killed. This is important to acknowledge: at the end of the Khmer Rouge rule not only had most of the Cambodian intellectuals been killed, but also the more educated cadres (Ayres, 2000). This contributes to quite a different situation compared to that of other post-conflict countries like Germany and Rwanda, where at least a reservoir of intellectuals remained to draw from when the rebuilding process was initiated. Secondly, in contrast with the Nazi regime, during which the Germans suppressed Jews, Gypsies and homosexuals, and the Rwandan Tutsi-Hutu conflict, the Khmer Rouge were Cambodians fighting Cambodians. This had and still has complicated consequences for the reconciliation process in Cambodia.

Education, one of the priorities under Sihanouk, was no longer a priority under the Khmer Rouge, but there were differences between regions. The relocated city dwellers were 're-educated' through physical labour in the camps. The original peasant folk remained living in villages. Children in Khmer Rouge strongholds received at least some form of education, whereas those in the camps did not receive anything more than basic writing and arithmetic, if they received any at all. One Khmer Rouge cadre said: 'Under our system, we don't need to send our young people to school. The farm is our school. The land is our paper. The plough is our pen. We will write by ploughing'. Another person said, about education in a favourable stronghold: 'There were schools and the teachers were well-educated...'. Educating activities that took place had an indoctrinating element in favour of the Khmer Rouge ideology. Revolutionary songs were sung. 
'Children were told again and again of the need to work hard, to protect the revolution by reporting on their parents and relatives, of the glories of Kampuchean socialism, and the danger posed by Vietnam', said an eyewitness about education at that time (Ayres, 2000, pp. 110-112).

One could argue that what people learned by experience during that time had much more influence on people's behaviour during and after the Khmer rouge period than formal education. Trust, for example, remains a difficult concept in contemporary Cambodia. There is often distrust between people who do not know each other well and unquestioned trust between people who have come a long way together. Some family ties and friendships are extremely strong if families or friends supported each other throughout the Khmer Rouge rule or in the camps in Thailand afterwards. For example, one of the interviewees survived as a three year-old because her older sister took care of her, after they were separated from their parents. Their bond is extremely strong. 'Nothing could cause a breach (between them),' she said (16 April, 2006). Examples can be found in organisational culture as well. If colleagues made it through the Khmer Rouge time together as friends, they tend to mutually support each other in decisions made in work. Even if it is obviously not a wise decision, the support for the other seems to be more important than the outcomes of such a decision.

It is sound to conclude that the Khmer Rouge, in their four years of rule, eliminated the old French-based education system. They tried to install a new egalitarian and completely self-sufficient nation-state that was hostile towards any serious form of education. Ayres (2000) described that the Khmer Rouge did so by pursuing failure in education in any possible way: education was withheld from many, and those who received education did so under extremely deficient circumstances: Teachers were poorly educated, if at all, buildings were in poor state or non-existent, and teaching and learning materials were absent. The children and teachers were overworked as they were undernourished, dislocated from their families and had to work half a day on the land before they could go to school.

Vietnamese forces defeated 'Angkar', or the Khmer Rouge regime, on January 7 1979. The regime left almost two million Cambodians dead out of a population of almost eight million (Ayres, 2000). Of those families who were reunited, people either returned to their hometowns or, as many did, fled to neighbouring countries. 


\subsubsection{Education during and after Vietnamese occupation}

After the defeat of the Khmer Rouge, Cambodia was found to be completely destroyed. The Khmer Rouge had eliminated the education system, civil services, the health sector, and had ruined Cambodia's agricultural sector. It also became clear that the country was depleted of its educated inhabitants. This latter fact is, as said, in contrast with other countries that suffered destruction by war, making the rebuilding process much easier. But in Cambodia, for example, there were only five lawyers left in the whole country. According to the 1996 Asian Development Bank Education Sector Study (ADB, 1996), the number of Cambodians with post-secondary education at the time was estimated at not more than 300 people. A 1984 Government report (in Ayres, 2000) stated that the Khmer Rouge regime murdered $75 \%$ of teachers, $96 \%$ of higher education students and $67 \%$ of primary and secondary school-aged pupils. The need for expertise was profound. In the reinstated Ministry of Education, the low level of qualified staff and lack of technical skills were extreme.

Data from three interviews with Cambodians who were enrolled in education just after the Pol Pot period describe how teachers were chosen from the adults who had survived. Because almost all intellectuals had been killed, people with a primary school grade were selected to teach in primary school, those with some years in secondary school were selected for secondary education, and those with some background in higher education were deployed at university (see also Interviews, December 2008; Ayres, 2000).

Advisory support by the Vietnamese during occupation was huge and has been estimated to have included between 2,000 and 12,000 personnel (Ayres, 2000). It reinforced anti-Vietnamese sentiments that date back long before Vietnamese occupation (Altner, 1999; Ayres, 2000; Verkoren, 2005).

The Vietnamese installed a socialist Government, consisting of many defected Khmer Rouge cadres. Education was used as a channel for spreading - and sometimes indoctrinating - young people with the new socialist state ideology. A student interviewed by Ayres (2000, p. 142) recalled the following: 'I remember writing in my exam: "The Mekong river will dry up and the mountains will be crushed before solidarity between the Kampucheans and Vietnamese disappears". But I did not believe it.' Interviews with the three Cambodians who studied during the Vietnamese occupation suggest that indoctrination was not 
always through the curriculum itself, but merely through activities around lessons. One interviewee tells how in primary school 'we needed to describe how we could be productive for 'our regime', what it meant for the Vietnamese, and for our leader' (interview, 12 December, 2008). Another interviewee recalled 'they wanted us to remember that Vietnam came to save Cambodia. On Labour Day we were all clean (washed) and were celebrating that day in the classroom' (interview, 12 December, 2008). An older interviewee studying agriculture at one of the universities during Vietnamese occupation remembered how 'student activists' were recruited to manage the other students: 'It was very hard at that time. You could not say what you thought, or the student activists would report you. If you had family living overseas, you would be monitored. I was not so sure what they tried to control, but I know it was difficult to say some things. It was also impossible to study English. Police would catch you. But political things were not really in the curriculum. Instead, we would have political meetings or conferences every two to three weeks in the morning, where they taught us about the relationship between Cambodia, Russia, Vietnam and PAC (Communist Party of Cambodia)' (interview, 12 December, 2008). Within the educational system, secondary students had to choose between learning Russian or Vietnamese, and learning English was forbidden.

The examples above show how Cambodians remained influenced and oppressed during Vietnamese occupation. Cambodia continued to be sealed off from the rest of the world and people were not free to speak their minds.

The new state ideology was only implemented in education to a limited extent, because of a 'conflict over ideas' within the Ministry, as described by Ayres (2000, p. 134). Only the higher ranks were in favour of the new ideology. The Khmer staff at the Ministry was far less convinced. Although many Government staff were not suited for their job responsibilities, many at the Ministry of Education had a predilection for the old French-based education because it was believed to carry considerable weight. Cultures with masculine characteristics - like Cambodia to a certain extent (please see further detail later on in this thesis) hold beliefs that value robust education (Hofstede, 1986).

The Vietnamese occupation of Cambodia ended in mid-1989, as a consequence of global political changes. The launch of the Perestroika - or restructuring - policy by then Russian President Mikhail Gorbachjev in April 1989 led to the abolition 
of the Soviet Union and the end of the Cold War era. Russia's interests shifted to internal affairs, leading to declining support for befriended overseas nations. The Vietnamese withdrew from Cambodia in September of that same year. In effect, these critical events led to the opening up of Cambodia to the world. But Cambodia needed to convince the West and the United Nations of its sincerity as a state. The country tried to improve its human rights profile by reinstating Buddhism as the state religion and abolishing the death penalty. According to Risse and Sikkink (1999), such instrumental implementation of human rights is the first step towards socialization of a state and a necessary step towards receiving international aid.

It is unclear how good or bad the education system was during and after Vietnamese occupation, although there is evidence that the quality of education was low. For example, after ten years of occupation only ten psychiatrists were trained in Cambodia (Verkoren, 2005). On the other hand, data from interviews with three Cambodians who studied during and after occupation show that teachers, although poorly educated and with a lack of teaching materials, worked hard to provide education. There was no corruption and primary and secondary school pupils also learned life skills in order to produce food. All interviewees recalled how hard everybody worked. Students and teachers came on time and rules were rigid. One interviewee classified the quality of education at that time as 'in general very good'.

In High School the instructional languages were Khmer, Russian and Vietnamese. The curriculum of the Agricultural University followed by one of the interviewees was completely in Russian and taught by Russian teachers. Students were taught Russian in a six-week preparatory program, which was hardly enough. She recalled that the curriculum at the agricultural university was not adapted for the tropical Southeast Asian climate: 'The knowledge of the Russian teachers was good, but the content (on farming) from their agricultural climate to ours was bad. They just took everything from their own 'bible' (handbook) and explained it to us. It was not adapted for the tropical situation, but it was as it was in Russia' (Interview, 12 December, 2008).

The retreat of the Russians and Vietnamese had at the very least some negative consequences for the education system: curricula had to be translated, adjusted or modified in order to meet the demands of a market-oriented economy. Russian and Vietnamese teachers were dismissed instantly, leading to a lack of teaching staff. Cambodian students replaced their teachers, without any consideration of their grades and quality (Godfrey, 2000). 
After the United Nations-led Paris Peace Treaty was signed on October 23 1991, the formerly fighting factions declared a cease-fire, although fighting continued in some parts of the country until the late 1990's (Peterson \& Petri, 2004; Taylor, Wheeler, \& Robinson, 1996; Verkoren, 2005). Despite the continued fighting, refugees could return to their homelands, a market economy was initiated and the influx of international aid started: Cambodia's isolation had ended. A constitution characterised by pluralism (recognition of a broad range of convictions) was installed and the first elections were held in 1993. The elections resulted in a 'two-state' Government: two Prime Ministers started to lead the country, with two supporting parallel power structures making and executing policy (Ayres, 2000; Verkoren, 2005). Despite the pluralistic constitution, both Prime Ministers tried to extend their legitimacy with the same notions of power and leadership, reinforcing hierarchy, ranking, hegemony and servitude, as their predecessors before the Khmer Rouge era. McNamara (1999) concludes that since the 1980s, decision-making by leaders in the Government had become a matter of faith that could not be challenged, and that it was merely political interest that had precedence over technical and good governing criteria. The policies for reconstruction were mainly Western initiated, but where the Khmer took control over projects they often led them in a different direction than the Western organisations had envisaged. Capacity building policies and models for development were formulated by foreign experts, stamped by the Government, but seldom adopted (McNamara, 1999, p. 165). It is sound to conclude that the UN and Western influences on promoting Western beliefs, for example in the constitution and working ethics on the one hand, conflict with traditional political culture and conceptions of power on the other. This made it difficult to create a modern state as envisaged by the international donors.

Again without attention to the educational quality, the new administration reemphasized expansion of the education system. Many of the same reasons for dropout and exclusion that had existed under the Sihanouk administration continued to prevail (Ayres, 2000; Tully, 2002). It is striking that during an informal meeting in 2006, a staff member of MoEYS explained that children were expected to work on the land when physically strong enough and that sometimes parents did not see the value of education when even the teacher had to refrain from teaching and work on the land to make enough money to feed the family (5 May, 2007). 
Similarly, Ministry of Education figures from 2001-2002 (see table 3.2; 'Enrolment percentages for primary education in 2002') show a decrease in enrolment from grade 5 to grade 6 of more than $8 \%$ for boys (NEFAC, 2003). Boys are enrolled with an average of $53.5 \%$, girls with $46.5 \%$. The Ministry explains the decrease in enrolment for boys with the onset of puberty and the work they can do. The lower enrolment rate for girls is explained by a lower desire to send girls to school when parents have to invest in education for their children. The net enrolment rate is worse for lower secondary education (16.4\%) and upper secondary $(5.4 \%)$. The distance from home to school is one of the main concerns, as students sometimes need to stay in dormitories and parents find such accommodation less suitable for their daughters.

Table 3.2 Enrolment percentages for primary education in 2002 from EMIS (NEFAC, 2003, p. 12)

\begin{tabular}{|lll|}
\hline Enrolment by Grade & Percentage Boys & Percentage Girls \\
\hline Grade 1 & 53.0 & 47.0 \\
\hline Grade 2 & 53.4 & 46.6 \\
\hline Grade 3 & 53.6 & 46.4 \\
\hline Grade 4 & 53.6 & 46.4 \\
\hline Grade 5 & 53.8 & 46.2 \\
\hline Grade 6 & 45.1 & 45.1 \\
\hline Total Enrolment & 53.5 & 46.5 \\
\hline
\end{tabular}

Since the Paris Peace Agreement of 1991, donor and aid organisations have been supporting Cambodia in the reconstruction process. It can be concluded that the contradiction between the donor-driven modernization of Cambodia and the country's tradition with its notions of power, hierarchy and leadership is a recurrent issue. This collision of values remains prominent in Cambodian economic, political and cultural life and keeps the dichotomy between change and changelessness alive. The country tries to pursue development in a culture that tries to hold on to its traditions. The state remains a function of (personal) power, as it did under King Sihanouk and corruption thrives. Verkoren (2005) argues that Cambodia can only make serious leaps forward in development if these old characteristics and issues that led to the conflict are resolved. She names the gap between rich city-dwellers and the poor country people, existing clientpatron relations, corruption and nepotism, as main issues to be solved. According to Verkoren (2005), education should play a role in creating trust and 
confidence in democratic politics and its institutions. Interestingly, she does not illustrate why education should play such a role and how this process could occur. Furthermore, the assumption of a democratic political system is a western bias in itself.

Junne and Verkoren (2005a, 2005b) warn that conflict may return to post-conflict areas, if the causes that led to the initial conflict are not resolved. Post-conflict development thus needs 'to be more than the reconstruction of a country, because it may lead to rebuilding the very structures that have given rise to the devastating conflicts' (p. 6). They stress that economic development alone does not prevent resumption of conflict, but development does. They warn that large numbers of unemployed males, continuing poverty and lack of perspective towards a better life could revive conflict. This is not an imaginary threat: only one out of every ten graduates in Cambodia is currently able to find work ('Cambodia's higher education', 2008), the same ratio as under King Sihanouk in the 1960s. Higher education is expanding rapidly, from 25,000 students in 2000 to 135,000 students in 2008. Several authors (Ayres, 2000; Junne \& Verkoren, 2005b) found that all levels of education are qualitatively low. Their findings are supported by three interviews conducted by the researcher in Cambodia in December 2008: the interviewees stated that teachers and professors are currently poorly trained and under-qualified, classes are crowded, students cheat, unacceptable numbers of students repeat grades and too many students drop out before completion of their studies; in short, control of the level of education in both public and private universities is low or lacking. They all thought that currently, education is poorer than it was when they studied, and were worried about the quality, the discipline in schools, and the corruption. They claimed that at that time (when they went to school), cheating during exams and the buying of diplomas were both impossible. One interviewee stated: 'During study and exam we did not pay, there was no corruption. Now that is different.' (12 December 2008).

Knowing that education is a condition for economic development, Cambodia tried to control the quality of higher education in 2003 by establishing a national University Accreditation Committee. The project was funded by the World Bank, but terminated when it found that the committee was not independent from the Cambodian Government ('Cambodia's higher education', 2008). This could be considered a missed opportunity to improve the education of the many young Cambodians who are enrolled in higher education. 
The legacy of war and occupation, a succession of regimes and being a people torn apart, was -and remains- tremendous. Education has been used over and over again for the purpose of legitimising regimes, and curricula have been used to spread ideology. After so many decades of indoctrination, and hence the suppression of critical reflection (Spiecker, 1991), it is hardly surprising that critical thinking skills are difficult for many Cambodians. Quality of education has always been low and students have rarely been challenged to become critical learners. With these characteristics, Cambodia entered a globalised world.

\subsubsection{Cambodia in the globalised world and the demands for education}

The world is changing rapidly. Merriam, Caffarella and Baumgartner (2007) point out that dramatic changes in demography, globalization and technological development strongly influence the agenda of adult learning, and thus of professional development.

Globalization is described as a movement of economic integration, of cultural homogenization, and of technical uniformisation. It is part of a long process that started after the Second World War, followed by the liberalization of the state that was then followed by neo-liberalism, which strove for individual liberty, free markets and free trade (Finger, 2005). Globalization processes have changed international relations tremendously: contemporary international relations are characterized by fast and extensively growing social networks with other nations and an ever extending flow of information within these relational networks. The impact of this process is enormous (Glastra, Hake \& Schedler, 2004). The effect of globalization, however, is that those countries that are already better off can accelerate and countries that lag behind are excluded (Merriam et al., 2007). Many have argued that globalization is much older; however the mutual reciprocity has changed in favour of Western dominance.

Finger (2005) asserts that the globalization process is Western-dominated, even in its cultural aspects: the global culture is a homogenizing process, but also promotes individualism. Globalization is responsible for the shift from a production society to a service society. But that is seen from the Western perspective, as non-Western countries like Cambodia took over most of the production activities of developed countries. In that sense, the change for Cambodia is more from an agricultural post-war society to an industrial one. Such differences have implications for professional development. Therefore, the starting point and needs in developing countries are different and should be 
addressed accordingly. However, the Western development agendas (based on Western experience and perspectives) determine professional development processes in developing countries. They often do so without acknowledging unique traditional forms of local society that can be completely inconsistent with Western conceptions. They also fail to acknowledge the fact that Southeast Asian countries evolve much faster and under completely different cultural preconditions than the West did (Mutz \& Benda, 2005).

Globalization also triggers counteracting processes. Finger (2005) points out that globalization can simultaneously generate many cultural reactions against it. Both are easily visible in Cambodia. Some Cambodians have changed their lifestyle towards a more Western pattern of consumption, occasionally exhibiting highly masculine behaviour contributing to a widening power distance. Many other Cambodians on the other hand, rich and poor, react to this by protecting their cultural identity, which is characterized by gentleness, kindness, politeness and respect for others. For example, many young Cambodians visit the Buddhist festivals and bring rice to more rural pagodas, which they know are less well supported.

Schön (1971) identified signs pointing towards globalization processes as early as the 1970s. He found that modern societies had lost their 'stable state' and had entered a state of continuous transformation. According to Schön, it is impossible to hold on to unchangeable constant beliefs about personal lives and society under such circumstances. The loss of the stable state had a disorienting and frightening effect on people in the West. It led towards moral uneasiness, anxiety, aimless violence, frenetic living and a general sense of confusion (Schön, 1967). These same issues can be identified in Cambodia, where people first lost their security due to ongoing warfare, and now seem to be losing the recently created stability because of globalization processes.

\subsection{CULTURE AND PROFESSIONAL DEVELOPMENT}

\subsubsection{War and the complexity of Cambodian culture}

Contemporary Cambodian culture is complex. It is influenced by it's long period of civil war and separation from the rest of the world. Many other countries in 
the region opened up to the world much earlier than Cambodia did, and adjusted some cultural aspects to become economic powers in the globalised world. Cambodian society, probably because of the need for stability as described in the previous sections, tends to hold on to its old beliefs and cultural ways, while at the same time trying to connect with the rest of the world. These cultural issues have important implications for capacity development in Cambodia, and are therefore important to understand. Many international experts involved in capacity development in Cambodia do not understand the context in which they work. This results in a gap between what organisations think they accomplish and what happens in reality.

Research on the impact of war on coping mechanisms and culture in Cambodia reveals that mistrust, fear and the inability to retain social relationships are widely spread, together with learning problems, memory loss, disorientation in space and time, sleeping problems, depression, apathy, aggression and other violent behaviour. Such problems and survival mechanisms as a way of coping can, according to Harmer (1995), manifest long after trauma took place and remain long after peace has returned. Another effect is 'somatisation', which is the expression of social distress through physical complaints and seems to be a common mechanism in societies where speaking about such distress is culturally inhibited. From observation it is obvious that Cambodians use somatisation as a way of channelling distress. Loss of planning ahead and inability to think beyond the day are also mentioned as survivor behaviours in war-torn societies (Harmer, 1995). Godfrey (2000) explicitly mentioned the lack of planning ability at all levels in Cambodia as a result of prolonged insecurity caused by civil war.

Unfortunately, little is known about the transfer of war trauma from one generation to the next. What is known is that younger Cambodian generations have lacked obvious role models during their formative years. Grandparents were missing in most families, where they should have fulfilled the roles of maintaining social harmony and supporting community networks. As they did not survive to support young families and these families had to find a way of surviving, children were usually left without adequate care (Harmer, 1995). Harmer speculates that the younger generations might therefore lack good parenting skills. Similar utterances can be widely heard in Cambodia. The cultural bias behind these pronouncements needs some attention. The view that children in Cambodia are not watched after and are denied their youth, as they need to work and take care of their younger siblings, is a very Western one: it is 
mainly in the West that children are freed from their responsibilities at home or towards the family. It is a misapprehension to victimize Cambodian children when they have to fulfil their role in or around their homes. For example, a photo exhibition by a young French photographer in the National Museum in Phnom Penh in December 2008, showed purposefully blurred pictures of Cambodian children working in their villages guarding the cows, selling water along the road and other such activities. The introduction informs the visitors that the older Cambodian generations deny young Cambodians their right to be children, as they have to work on the fields, and take care of cattle and siblings. When asking some colleagues (two unrelated 36-year old Cambodians on two different occasions) about their youth, they stressed the responsibility they had, also as children, to contribute to daily family life and how much they enjoyed guarding cattle and working on the fields to harvest. One described it as the best time of his life and told a story about how the cows tended to run into the mud and how much fun they had getting the cows out again. The exhibition showed an exact picture of such an incident. The interpretation though, was a completely different one, namely a form of child labour. This example shows how easily cultural beliefs can shape perceptions and how important it is to ensure that conclusions are true to the context and the beliefs of that context.

Another point that needs to be approached with caution is Harmer's remark about Cambodian children lacking 'adequate' care. It is easy to observe that Cambodian children almost never whine and are extremely polite. Somewhere in their upbringing these values are instilled in the majority of children, making it hard to argue that they are not raised adequately, whatever that term may imply.

\subsubsection{The 5-D model for cultural differences}

The 5-D model for cultural differences (Hofstede, 1986; Hofstede \& McCrae, 2004; Gervedink Nijhuis, 2008) gives a good overview of important aspects to understand when working in culturally different contexts. Although Hofstede's research conducted in over 50 countries is dated, the dimensions he introduced are important to form a better understanding of why some things work and others do not. Cambodia is not part of the study, as it was at war during the time the Hofstede's study was conducted. Thailand is the country closest to Cambodia in both geographical and cultural senses and included in the research. But in the 1980s Thailand developed into one of the Asian tiger economies, with enormous 
consequences for the economic state of the country, and thus for at least some of the cultural aspects described by Hofstede (Hofstede, 1986; Hofstede \& McCrae, 2004). Therefore additional specific Cambodian research was done by the researcher to describe Cambodian culture along the five dimensions of Hofstede. This was not an easy task, as there has only been limited social research done in Cambodia before the Khmer Rouge era (Harmer, 1995) and not much thereafter.

Hofstede initially described four dimensions, which were later extended with a fifth dimension (Hofstede, 1986; Hofstede \& McCrae, 2004; Gervedink Nijhuis, 2008):

1. Individualism/Collectivism: This dimension describes the units for which members of a society take responsibility. Cultures that are individualist see personal interest and interest for immediate family as the unit of responsibility, whereas collectivist societies embrace larger groups of people that are related or interrelated, in their 'in-groups'. Individualist societies are loosely integrated, collectivist ones tightly.

2. Power Distance: Power distance reflects the extent to which less powerful people accept inequality in power as being normal. The smaller the power distance, the less people accept these differences in power. The larger the power distance, the more people accept these differences.

3. Uncertainty Avoidance: Uncertainty avoidance is the extent to which people are made nervous by situations that they perceive as unstructured, unclear or unpredictable, and to what extent they maintain strict codes of behaviour and beliefs in absolute truths to counteract uncertainty.

4. Masculinity/Femininity: The masculinity-femininity dimension is based on the social roles of men and women, associated with biological facts between the two sexes. It is important to understand that for both types of society, males remain dominant in political and work organisations. Feminine societies tend to demonstrate more overlapping social roles for men and women, for example being less ambitious or competitive, and paying more respect to anything that is small, weak and slow. Feminine countries usually have stronger social security networks. Masculine societies on the other hand, aim for maximum distinction between male and female roles. Men in these 
societies tend to be highly ambitious, competitive and respect everything that is big, strong and fast. There is less care for the weak, old and those in need of support. Students in more masculine societies tend to overrate their selfperformance, which is the opposite in more feminine societies.

5. Long-term/Short-term orientation: Societies with a long-term orientation are more frugal and persevering, whereas countries with a short-term orientation try to respect tradition, have a stronger social commitment and prevent people from losing face.

The following application of the 5-D model for cultural differences to Cambodia is based on three additional sources of data: an introduction from a feasibility study conducted by Harmer in 1995, findings of focus-group discussions of a working group established in December 1994 conducted with the participation of 322 Cambodians of various ages, education levels and residence (In Harmer, 1995), and a small survey conducted by the researcher in December 2008, with 37 Cambodians. It is acknowledged that the outcomes of the focus group discussions are based on people's recollections of a period 25 years before the time of interviewing. It is important to understand that the older data are more than ten years old and might not be reflected by the findings of the 2008 survey, as Cambodia has undergone many changes since then. At the same time, all Cambodians of working age were raised in the 1990's and probably were influenced by the situation described by the older research. The small 2008 survey was specifically conducted for this research and consisted of randomly selected subjects, agreeing or disagreeing with 16 statements (in both Khmer and English) which reflected Hofstede's five categories. The conclusions below were discussed with Cambodian and expatriate colleagues who have lived in Cambodia for a number of years, to double-check the validity of the findings.

\section{Individualism/collectivism}

Harmer (1995) describes the extended family (nuclear family and other relatives living nearby) to be of great importance before the Khmer Rouge era. During their regime, the Khmer Rouge forced Cambodians into 'enforced collectivism', in which traditional social, economic and cultural relations were disrupted (p. 3). After the Khmer Rouge period, extended family members became more important, as so many families were depleted of their members. 
The findings of the 1994 focus-groups conclude that Cambodian society after the Pol Pot regime was less collectivist than before the Khmer Rouge era. That conclusion was based on the fact that people experienced greater poverty, and less solidarity with others than with immediate family. People were thought to be less willing to enrol in activities benefiting the wider community. Families were more splintered in 1994 than before the Khmer Rouge time in order to pursue income. Male/female relationships were less stable and moral standards seemed to be in decline.

The 2008 survey shows that Cambodians have a strong preference for immediate family and do not trust people from the outside circle.

Summarizing, Cambodia is collectivist but with a smaller 'in-group' than other Asian countries in the region. Cambodians seem to care less about people belonging to the out-group, which is larger than before. There is suspicion towards Cambodians from the outside. Cambodian society outside the family is loosely integrated.

\section{Power distance}

The focus-group findings indicate that politicians in 1994 were mostly interested in power and that they were greedier than before. Participants agreed on the perception that there was more corruption and exploitation, leading to run-down civil services and economy (the latter is disputable now, with a yearly economic growth rate of a steady 9\% over the last ten years (Asian Development Bank [ADB], 2008)). It was felt that before 1970 politicians were less corrupt and that they strove for good Governance by providing services like health care and education. As mentioned earlier, this positive image is rejected by Ayres (2000).

Harmer (1995) postulates that 'traditionally, Cambodian society has emphasised preservation of the status quo, a tolerance of suffering and injustice and a conformity to higher authority' (pp. 10-11), and that this is connected with Buddhist notions of acceptance of suffering and having been oppressed for so long. Cambodia has always had a 'patron-client system' emphasizing reciprocal relationships. She further implies that Cambodians may accept living with the burden of suppressed emotion on cultural and historical grounds. This, however, is arguably a Western bias that it is preferable for people to openly express their emotions, instead of hiding them inside.

The 2008 survey shows that Cambodians believe that 'people with power' have more rights than they themselves have $(68 \%)$, and more than $97 \%$ of the subjects 
accept that difference. On a scale of 0 to 1 , the total group of subjects scores 0.18 , indicating a large power distance. Additional information on power distance comes from research by Godfrey (2000), who found that power in Cambodia is usually not delegated, and remains concentrated at the highest levels. Furthermore, selection for promotion and enrolment in capacity development programs is usually based on (political) loyalty and seniority, rather than aptitude. All sources indicate that Cambodia has a large power distance. Harmer (1995) legitimately asks the question to what extent patrons fulfilled their obligations, triggered by indications of exploitation of the powerless. Remarkably, the 2008 survey found that $32 \%$ of the Cambodians say that they do not feel that people with power should have more rights, but $97 \%$ in the end accept these differences. This might be an indication that conflicts are not at hand. Some of the people asked to comment on these findings think that many Cambodians are willing to accept inequality of power, but expect peace and political stability in return. In general, Cambodians do not like to talk much about this inequality of power distribution. In that light it is notable that the name for people with power in Khmer is 'manoes Thom' which literally means 'big person' and is similar to the term 'big shot' in English.

\section{Uncertainty avoidance}

Harmer (1995) argues that most Cambodians accept uncritically their Buddhist religious dogmas. Coping mechanisms from previous wartime and trauma such as mistrust, passivity and preservation of self-interest as described by Harmer are difficult to classify but fit best under uncertainty avoidance, as they contribute to extension of the status quo.

The focus-group discussions concluded that there was more lawlessness, violence and anarchy in 1994 than before. People were more afraid for their personal security than before the Khmer rouge, indicating stronger uncertainty avoidance. On the other hand, however, traditional practices were perceived to be less celebrated and people were thought to be less superstitious and more open toward Western culture than before. These are indications of reduced uncertainty avoidance.

The 2008 survey shows that the overall group scores .30 on the scale for uncertainty avoidance, indicating high uncertainty avoidance. Almost $65 \%$ of the subjects never question their religion, more than $86 \%$ believe that a good Cambodian respects tradition and $70 \%$ do not like unpredictable situations. The 
same percentage $(70 \%)$ of Cambodians do not like unclear or unstructured situations, showing that fear of situations that are not known, is prevalent.

Additional information comes from observations conducted between 2006 and 2009. These observations show strong religious commitment. The vast majority of Cambodians, young and elderly, are highly involved in religious ceremonies and customs.

The findings indicate that Cambodians demonstrate high uncertainty avoidance. There is fear of unclear, unstructured and unpredictable situations. The majority of Cambodians obeys strict codes of behaviour and believe in absolute truths. Absolute truths can also come from higher ranked people, and will be obeyed by everybody. But with the Westernizing trend of society, it seems that people are starting to lose some of their uncertainty avoidance. To what extent this is happening remains to be seen. For now, Cambodians in general are characterized by high uncertainty avoidance.

\section{Masculinity/femininity}

The focus groups agreed on the perception that before the Khmer Rouge time Cambodians were kind and gentle. People were proud of their country and nation. In 1994 Cambodians were still thought to be kind and gentle, but were also selfish, greedy, jealous and violent.

The 2008 survey shows that the average score is 0.63 . This indicates a slightly feminine society. This is partly influenced by the fact that more than $78 \%$ of the subjects believe that men and women have the same rights, and $70 \%$ believe that Cambodians take care of their old people. The other indicators are more masculine: $78 \%$ of the subjects prefer a big eye-catching car, and $40 \%$ thinks Cambodians are ambitious.

Over-estimation of personal capacity is common. A training needs assessment conducted in the Cambodian health sector in 2006 shows that many trainers over-estimate themselves: almost $90 \%$ of the national trainers rated themselves as 'above average' and 'strongly skilled' as trainers, but training results remain weak (Domrei, 2006). This is confirmed by observations performed by the researcher of a training-of-trainers workshop for a national health volunteer program. Participants were asked to agree or disagree with statements about training. If participants disagreed they were asked to remain standing, if they agreed they were asked to sit down. One of the statements was 'I know everything about participatory activities and use them in my training courses', $85 \%$ of the participants agreed by sitting down. Observations of a large number 
of training courses and workshops conducted between 2006 and 2008, however, contradict the positive perception of participatory facilitation skills of trainers by themselves: they show that almost all trainings are top-down, knowledge-based and only a minority of trainers is able to use participatory techniques.

Cambodian society is mainly feminine but also shows some strong masculine characteristics. Masculine characteristics (big cars, violent behaviour) are demonstrated mainly by the rich and powerful. Feminine characteristics on the other hand, like politeness, correctness and kindness are demonstrated by everybody.

One remark about bias should be made here. The outcome of the question about Cambodians taking care of their elderly strongly depends on interpretation. Homes for the elderly do not exist in Cambodia and the fact is that many older Cambodians do not have a place to live. Many are widowed and deprived of family and are taken care of by local pagodas (religious community centres). That can be seen as a way of taking care of the old, but it is surely not the same as taking older people into one's own home or receive them in Western-style elderly homes.

\section{Long-term/short-term orientation}

In the 1994 focus-group discussions presented by Harmer (1995), participants agreed that many people in 1994 could not afford to send their children to school. If a choice had to be made between a girl and a boy, it would usually be the boy that was sent to school, although there was recognition that education is also appropriate for girls. In general, people were thought to be less hard-working than before Pol Pot, although there was more interest in modern technology than before. Another conclusion of the focus groups was that Cambodians showed symptoms of aid-reliance, dependency, passivity and hopelessness (the latter two seem to be at odds with the actions the vast majority of Cambodians constantly makes to meet their daily needs and alleviate poverty).

The 2008 survey shows that Cambodians are acting for the short-term, although almost $73 \%$ says they would rather have a difficult time for some years, in order to have better times later.

Additional information from observations conducted between 2006 and 2008 shows that many young Cambodians follow courses in addition to formal education or jobs. They are highly motivated to gain more 'experience' and knowledge, in the hope that they will benefit from those efforts in the future. 
Cambodia shows a short-term orientation. Where neighbouring countries have two or even three rice harvests a year, Cambodia has only one. Data from informal discussions show that Cambodians themselves attribute this to shortterm thinking: for many it is unclear what tomorrow will bring, so people tend not to think too far ahead. Although Cambodians seem to be living from day to day, many younger and ambitious Cambodians have more elaborate ideas about their future and tend to have a more long-term orientation. These are mainly citydwellers who work or study during the day and continue further study in the evening, and do so in order to improve the quality of their own lives and that of their small in-group.

\subsubsection{The influence of culture on learning}

Additional research by Hofstede in the 1980s looked at the influence of culture on learning. His definition of culture includes the importance of learning:

'The collective programming of the mind which distinguishes the members of one human group from another' (Hofstede E McCrae, 2004, p. 58).

According to Hofstede, learning always takes place within a cultural context and includes beliefs and assumptions of that society. Different cultures can have different definitions of beliefs, assumptions and learning relationships. Hofstede (1986) identifies five of these learning relationships within four human institutions, which are depicted in table 3.3.

Table 3.3 Adjusted overview of human institutions and corresponding role pairs or learning relationships (Adjustments in italic) (Hofstede, 1986)

\begin{tabular}{|l|l|}
\hline Institution & $\begin{array}{l}\text { Role pair/ learning relationship } \\
\text { Parent-Child } \\
\text { Family }\end{array}$ \\
\hline School & $\begin{array}{l}\text { Teacher-Student } \\
\text { Teacher-Teacher } \\
\text { Student-Student }\end{array}$ \\
\hline Job & Boss-Subordinate \\
\hline Community & Authority-Member \\
\hline Intercultural community & Foreigner-Local \\
\hline
\end{tabular}


Within the family institution Hofstede describes the parent-child and the manwoman relationships. In school, he identified the teacher-student relationship, and on the work floor the boss-subordinate relationship. The final learning relationship is found within the community and is described as the authoritymember relationship.

Analyzing Hofstede's work and connecting it to the development context, it would be helpful to introduce a fifth learning institution and three more learning relationships to the model presented. The first two learning relationships should be added to the framework within the school institution (printed in italic). According to the paradigm of life-long learning, people learn continuously: teachers learn from other teachers, and students learn from other students. The third additional relationship is proposed for the Foreigner-Local learning relationship, which could be placed in the community institution, but preferably forms an institution of its own that could be described as the 'intercultural community' institution. Hofstede incorporated this relationship partly in the Teacher-Student role, but that seems too limited, as it implies only a one-way direction. The foreigner also learns should learn - from the local colleagues he or she works with. An additional argument for placing the foreigner-local relationship within its own specially designated 'Intercultural Community' institution is that this relationship can be found in any institution, not just in schools.

The foreigner-local relationship is not an abstract notion. As is shown in this chapter, foreign assistance in Cambodia is extensive. These experts are supposed to supply the Cambodian Government with expertise that is lacking, either with, but most often without an explicit capacity building role. They should work together with Cambodian staff members and such collaboration makes cross-cultural adult learning an important factor for success, also within Government structures.

Foreign advisers usually approach development from their - often Western perspective, contributing to misapprehensions between foreign aid organisations and local Governments. Cultures do have different priorities that are also reflected in Government structures. Pinto (2000) represents these different cultural priorities using pyramids based on Maslow's hierarchy of needs (see figure 3.1). He distinguishes between individually-oriented and group-oriented societies. The top of a pyramid represents the highest priority in a society. 


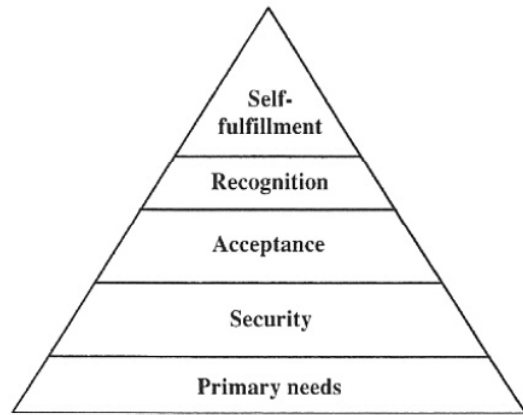

Individually-oriented Societies

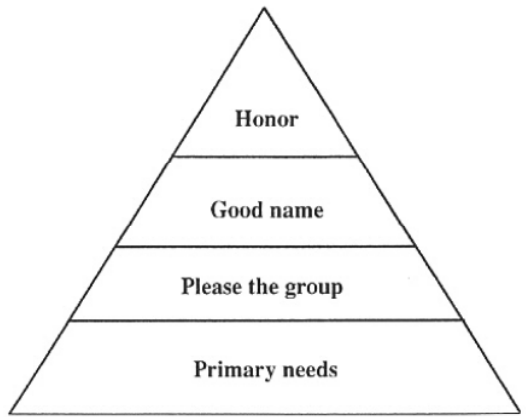

Group-oriented Societies

Figure 3.1 Different priorities of societies. (Pinto D.; in Junne \& Verkoren, 2005a, p. 10)

Group-oriented priorities usually are more applicable to the culture in Cambodian Government structures. It is important to be aware of these differences when working within the Ministry.

\section{Culture in Cambodian Government structures}

Much of the culture within Government structures follows the cultural description of the previous paragraph. Power distance, uncertainty avoidance and collectivism are important cultural factors within the Ministry and also important reasons why capacity building is sometimes not effective. Teaching people how to think critically and take responsibility in their job, for example, is in vain when a higherranking Government official decides in the end how things will be done anyway. Fear of losing face or causing somebody to lose face are important to avoid, but also leads to staff not speaking their minds because of these hierarchical issues. Culture within MoEYS is changing, however, with more and more young people coming in and ambitious plans being developed. This change is interesting and challenging for all people involved. It also provides more opportunities for participatory and learner-based professional development activities.

\subsubsection{Learning in a changing society}

Cambodian society is rapidly transforming into a more modern one and by doing that, the society has clearly lost its stable state. The country is developing at a high pace, with high economic growth rates. As has already been mentioned, the economic development has cultural consequences: a more modern society model is needed to keep pace with the economic development. Economic participation of women is necessary, changing the role of women in society. People have more 
money to spend, changing the needs of families. People need to trust each other more, as not all functions can be carried out within families anymore, but are outsourced to, for example, insurance companies taking over the care of the sick, and banks taking care of savings. Many Cambodians are suspicious of these 'anonymous' organisations.

Education, health care and infrastructure need to be improved and the role of the Government is changing. Relations between schools and local and central Government are changing accordingly. These processes of change are frightening for many Cambodians, who have learned that change is usually for the worse, not for the better. Societies with high uncertainty avoidance rely strongly on stability beliefs. People suddenly departing from the accustomed norms of behaviour make other people insecure. A loosely integrated society like the Cambodian one under such pressure of change is in danger of unbridled and uncontrolled change.

Adult education usually reflects the socio-historical context (Merriam et al., 2007). This is also the case for Cambodia. It is important to understand that all the learning relationships described by Hofstede (1986) were disrupted during Cambodia's recent violent past. The recovering system is influenced by the effects of that same past. For instance, teachers and Government staff are educated to a low level, explaining the huge demand for capacity building and input by foreign experts. Many of the training endeavours are simplified quick fixes without being embedded in a coordinated organizational development program.

The learning relationship between foreigner experts and Cambodian staff is important at this point. It is more complex than just a teacher-student relationship. Although many Cambodians still see the foreign expatriate as being an authority that is always right, more and more perceive foreigners as colleagues, which turns the relationship more into that of collaborating partners. From observation it seems that in some cases the relationship even turns into a boss-subordinate one, in which the boss is not necessarily the foreign expert.

Knowing that globalization and capacity development are Western agendas, led by representatives of the West, moving incredibly fast to 'improve' the world, it is not surprising to see that many developing countries or countries in transition cannot keep pace with the globalization process. The inability of developing 
countries to keep pace with Western demands or share the latest priorities of the West, sometimes leads to loss of funding and loss of income for the local Government. Some examples of Western development priorities in the Cambodian education sector are currently the Education For All platform (Dyer, 2001), the interest in ethnic minorities in rural areas (in general) and the agenda for Child Friendly Schools (UNICEF) as one of the implementation strategies of Education for All. Many of these programs are being implemented at the same time, each with slightly different points of view and targets, in a system that is still under construction, where teachers earn salaries that are extremely low and the Departments and offices responsible are understaffed. Almost daily, the Ministry receives delegations of non-governmental organisations (NGOs) and donors who wish to implement something somewhere in the education sector, usually through short-term training, as mentioned by Finger (2005). Real coordination seems difficult, although MoEYS worked on a capacity building multi-year plan (Berkvens, 2007a), funded by UNICEF, to try to coordinate organisational capacity building. This project will be discussed later in this chapter.

It is interesting to compare Cambodia and Thailand at this point, because there is a link between education, learning and destabilizing events like civil war on people's lives. Learning relations in Thailand were never disrupted as they were in Cambodia, ensuring the passage of culture from one generation to the next. In many cases, these relationships are still missing or are extremely weak in Cambodia. Secondly, Thai economic standards are much higher and include a working tax system, providing more space for individualism and smaller uncertainty avoidance. Thirdly, a middle class evolved in Thailand over an extended period of time in a fairly democratic climate. People feel relatively safe and secure (Hofstede, 1980), whereas people in Cambodia experience huge power distance and fear any instability that might occur. Chapter Four of this thesis describes favourable conditions for optimal adult learning and professional development. Feeling safe and being able to act without fear are key to successful learning. These conditions seem to be under pressure in Cambodia.

One example is reluctance to ask questions. It is easy to observe that Cambodians do not tend to ask questions during presentations and it is suggested that asking questions in schools is discouraged because of fear of losing face. Fear of losing face is often mentioned as a typical Southeast Asian trait. The interviews with Cambodians who were students during Vietnamese occupation shed a more 
subtle light on this matter. One interviewee described that the fear of loss of face as more a fear of doing wrong, than a fear that they might be putting the teacher in a position where the teacher might lose face. According to the interviewee, the fear of doing wrong comes from the Khmer Rouge period, where it was dangerous to express opinions, even in front of family members. That fear has developed into the fear of being laughed at, for example, when a question might be seen as stupid. Younger generations, from 1986 onwards, he suggests, do not have so much fear and have the courage to ask questions: 'But many of us (older people) still can't ask questions'. Ironically, right after the Khmer Rouge period and because of the lack of teaching and learning materials, learning happened through dialogue and oral dissemination. One of the interviewees described how asking questions was a sheer necessity during his studies, as there were no resources to learn from, and everything was disseminated orally and through dialogue: 'we shared a lot of our thoughts'. Teachers encouraged students to ask questions. He compared it with the current situation, and concluded that it is now more difficult to ask questions because teachers try to motivate students to actively consult currently available resources like books and the internet. The third interviewee thought that some teachers at present are afraid to answer questions 'because students belonging to high-ranking families can do bad' (interview, 12 December 2008). All interviewees said that fear of losing face might lead to teachers not allowing students to ask questions, but it is important to note that the interviewees only said this after the interview probed.

The findings presented above show that the fear of asking questions is partly linked to the fear of 'losing face'. When using participatory approaches and during collaborative activities, the 'fear' factor should definitely be given serious consideration, as it remains an important factor in the daily life of many Cambodians.

It is also important to understand that multi-faceted inequality (through hierarchy, fear, client-patron system etc.) in Cambodian society is much older than just the Khmer Rouge time. Many aspects already existed in the Angkorian empire. The state has always been oppressive and legitimized by the people being exploited by it. The oppressed were taught to respect the hierarchical system and maintain the correct relations between members of society. What people experienced during the Khmer Rouge regime changed or strengthened Cambodian customs and culture. This is still reflected within the cultural dimensions explored in this section. The cultural issues described in these 
sections are important factors to take into account when developing educational interventions. These notions are incorporated in the design principles that form the basis of this research.

\subsection{Professional DeVELOPMENT IN CAMBOdia}

\subsubsection{Aid and professional development}

In 2002, \$ 31.4 million was spent on training to support the Cambodian Government as a whole. \$ 8.1 million was reserved for broad multi-sector training, another \$ 4.7 million was spent in the education sector only. In 2007 the amount of foreign aid had risen to \$ 689 million. In 2003, the Ministry of Education alone, received 958 person months of assistance, the equivalent of 79,4 full-time positions. $72.8 \%$ of current professional development activities to strengthen capacity in the Ministry take place in Cambodia. Short-term training courses (up to 5 days) and workshops are the most common form of capacity development activities. Follow-up takes place in $47 \%$ of the projects, monitoring and coaching in 24\% (Beynon et al., 2004; 'Cambodia seeks foreign aid', 2008; Godfrey, 2000; Siddiqui, Strickler \& Vinde, 2004). The figures for follow-up, monitoring and coaching should be treated with caution, however, as these terms remain undefined in any of the documents, what is actually happening is not investigated and observations show that people are not familiar with these activities. Monitoring, evaluation, follow-up and coaching are often seen as the same thing. More often than not, none of these activities is carried out because of constraints (impassable roads, no budget, time constraints, holidays), while plans and evaluations show that they will be or are executed. According to interviews carried out by Siddiqui et al. (2004), short-term training courses, seminars and workshops often serve as ways to supplement salaries.

Beynon et al. (2004) analyzed five capacity development programs that were executed in the Ministry of Education between 1994 and 2004. The analysis provides useful information for future professional development interventions. First of all, they found that skills development in the Ministry usually took place through theoretical training, but that a combination of training and mentoring as was done for the Department of Finance and other Departments using the Education Monitoring and Information System (EMIS), was more successful. 
Secondly, selection of trainees was an issue, as a training program from 1999 to 2004 showed: coverage was 142\%, leading to the conclusion that many people had been trained twice. This was partly due to the inability of the organisation to select the appropriate trainees, but also based on the financial benefits provided to trainees in the form of 'per diem' payment that was also received twice. A third finding was that skills utilization rates went up when the training content was simple and easy to understand and the training was based on local realities instead of how it should be according to foreign theory: $70 \%$ of the schools who had followed the 'information-based school management' training in 1999 developed detailed annual plans.

Another example of a long-term capacity building program is the UNESCO/UNDP-funded project for improving Human Resource Management (HRM) in MoEYS in the 1990s, as described by Altner (1999). It is one of the most advanced attempts at professional development in the Ministry. Fifty participants were partly trained overseas to teach 12,000 participants in-country. The education levels of the fifty trainees selected to become trainers was well above average. The tendency to invite already advanced staff to take part in big professional development projects is also mentioned for developed countries by Kessels \& Keursten (2001), and thus does not seem to be a problem that is limited to the Cambodian or development context. It does, however, limit the group of beneficiaries to a small number of people, instead of strengthening the organisation as a whole. It seems that effective dissemination after a course hardly ever takes place within the Cambodian context (observations from 2006-2008).

The Training of Trainers (ToT) for the UNESCO/UNDP project was conducted overseas by three regional institutions. The course provided by one of these institutions was too advanced for the Cambodian trainees. The training course provided by one of the other institutions was badly organised and delivered mainly theory without any form of practice. These two examples contradict the widespread belief that regional training facilities have a better understanding of regional contexts and are able to provide tailored courses. This conclusion is supported by a quote of a Government staff member interviewed for this research in 2006, after returning from a training about education in Thailand: 'It was nice ... but we do not have all that (materials) and sometimes I did not even understand'. An additional ToT for the UNESCO/UNDP project was provided in Cambodia, with attention to adult learning theories, learner centred teaching, icebreakers 
and interactive methods. Unfortunately, the effect of this module was not evaluated, making it impossible to conclude any more than whilst modern learning theories and teaching methods have been introduced, the extent and effect of these remain unclear.

The training modules described by Altner, for the 12,000 participants in Cambodia, were evaluated with evaluation forms. Altner (1999) is satisfied with the outcomes, as participants evaluate almost every item very positively. He also notes that 'nearly all participants indicated that they would like to participate in another course in the future' (Altner, 1999, p. 192). Outcomes of evaluations in Cambodia, however, should be interpreted with caution. Personal experience shows that such Likert-scale evaluations in Cambodia do not provide a lot of information, as: a) Not all participants clearly understand the questions or are familiar with such evaluation forms; b) Cambodians tend to give answers they think the facilitator wants to hear; and c) Cambodians are extremely eager to learn and extend their experience. In some cases, training is also a means to top up a person's salary, as a 'per diem' payment is usually provided. It is, therefore, unlikely that people will say 'no' to further training or capacity building.

In short, the long-term outcomes of the UNESCO/UNDP project remain unclear. The intended follow-up was never provided and the more systematic approach, including status, career prospects and level of salaries, which the project strove for, was not adopted. The specially installed office, which was responsible for ongoing training, was abolished and with it the formal anchoring of the expertise gained from this project.

Professional development in the Cambodian Government is characterised by a lack of coordination and driven by foreign donors and aid organisations. Donors have taken over the funding of civil services like education, health, welfare and rural development. Aid is often not contextualized, although a standard, onesize-fits-all approach is known not to be effective (Altner, 1999; Junne \& Verkoren, 2005b). The uncoordinated interventions of international organisations lead to low ownership by the Cambodian Government and its continuing dependency on international aid. Training happens in a haphazard way, leading to many overlaps in capacity building endeavours (Beynon et al., 2004; Godfrey, 2000; Siddiqui, Strickler \& Vinde, 2004). 
A 1995 World Bank model of capacity development phases (Godfrey, 2000, p. 48) identified three successive stages, each estimated to last five years. Foreign aid workers carry out most of the tasks during the prerequisite phase, as the capacity of local staff is too limited. Prerequisites for effective capacity building include a stable environment, coherent rules, reduction of the size of the civil service, implementation of wage policy and incentive structure and the conducting of training needs assessments in order to develop capacity building plans. This phase is followed by the capacity-building and institutional development phase, where prerequisites should be in place to build capacity of local staff. The final phase is the consolidation phase, in which the Government should have advanced enough to manage its own development. A Government official interviewed in 2004 by Siddiqui, Strickler \& Vinde (2004) said that Cambodian counterparts had taken over the task of developing and managing projects, indicating that, at least in their experience, the transfer of knowledge was beginning to show effects. According to the 1995 World Bank model, Cambodia should have been ready for the shift to the second phase in 1999 and should have been aiming for the transition to the final phase later. Calls for a 'sector-wide approach' indicative of that phase had already been raised at that time by international aid organisations. The reality however, was that in 1998 none of the prerequisites mentioned by the World Bank for such a transition were in place, although international donors were treating Cambodia as if it already was. Only some of the prerequisites mentioned by the World Bank in the 1995 model, like the development of capacity building plans based on training needs assessments, and a more stable environment, had been met in 2008. Even now, the size of the civil service is still expanding and appointments are merely made on political or financial grounds.

Most multi-lateral organisations like UNICEF try to use the Government structure to carry out projects. Many programs supported by a specific country (bilateral programs) and NGO interventions, however, bypass Government structures, thus adding to the uncoordinated patchwork of capacity building. In an attempt to counteract this undesirable situation, sector-wide approaches have been launched within the Cambodian Government system. These approaches, however, belong to phase 3 as described by the World Bank, while some parts of the Cambodian Government are still depending on foreign assistance to carry out tasks (phase 1), or rely on extensive capacity building as described by phase 2 . 
The 2000 study of Godfrey concludes that technical assistance projects appear to have a good record for the development of individual capacity of Cambodian staff, but are extremely poor on institutional development. Beynon et al. (2004), however, conclude that neither is strengthened, at least not in the Ministry of Education, where 'all too often these (programs) are implemented without requiring the systematic transfer of skills either to their national counterparts, or on the broader vision of building up national capacities for sustained skills' (p. 16).

The emphasis in international development is currently on capacity strengthening for decentralization. What is to be decentralized, however, remains unclear (Beynon et al., 2004). Decentralization is a Western-driven process being implemented by many international aid organisations in several developing countries that aims to increase good governance through delegating responsibilities to regional, provincial or district layers of the Government structure. In many cases, this is pursued without complete knowledge of local dynamics and ignorance of the historical context, thus often leading towards failure in empowering lower levels within Government structures. Local realities then turn out to be stronger. Pursuing decentralisation without understanding and respecting local culture and history is questionable, often leading to gaps between the reality and what is developed and described on paper.

\subsubsection{Donor influence}

As mentioned earlier, external assistance to Cambodia is huge. Close to 2,000 national and international NGOs support Cambodia, which allows questions about their efficiency (Verkoren, 2005). The excessive number of organisations seems to have a crippling effect on the development of Cambodia: organisations often do not seem to work together, sometimes even working in opposite directions, and their demands for support seem to overload the Government and its staff.

The low level of development in Cambodia, with its specific constraints, and the large amount of aid, places Cambodia in an exceptional position (Altner, 1999; Godfrey, 2000). Godfrey (2000) concludes that the scale of aid is such that it distorts the economy' (p.123). The education levels are extremely low and the recovery process is slow. A 2004 framework for capacity development analysed the capacity building practices of Cambodia's external partners, in an attempt to 
assess the effectiveness of the aid provided (Cambodia Development Resource Institute [CDRI], 2004). The report identifies the low levels of trust, unwillingness to work together, excessive bureaucratisation, extensive reliability on international experts, unsustainable salary supplementation of Cambodian staff and officials, predatory competitive recruiting practices of local staff by donors and the emphasis on short-term programs, as the main reasons for the lack of success of capacity development projects in Cambodia.

A consultative group meeting in Phnom Penh discussed how to develop more effective partnerships for development in more detail (Altner, 1999), adding to the points also mentioned by others. The participants identified a variety of reasons for the lack of progress in capacity building.

Firstly, coordination between international organisations is weak. This leads to projects that 'pull in different directions' with the Government in an awkward position in between. Although projects using the existing Government structures will be more successful in the long term, many international organisations establish parallel systems when changing existing structures is found to be difficult (Junne \& Verkoren, 2005a). Aid organisations tend to pull their staff out of Government bodies with salaries that are impossible for the Government to compete with: A 1999 example indicates that a former civil servant went from a salary of $\$ 20$ to $\$ 500$ per month. This puts a burden on the existing pool of educated Khmer, while organisations compete for qualified personnel that should be working with the Government. Some Government officials and donors admit that there are too many projects. The funding of Ministerial activities depends partly on the donations of international organisations. Sometimes projects with contradicting objectives are implemented at the same time, causing confusion and creating situations destined for failure. Confusion is caused by contradictory demands by donors. A wonderful example is the push by international donor organisations for more participatory and learner-based methodologies for training in the Cambodian civil service, while the same donor organisations still operate the 1-dollar-per-participant-per-training rule for stationery, providing the same amount of money per participant to buy stationery for a one-day training session and a ten-day workshop. With such limited resources it is almost impossible to introduce modern participatory approaches.

Secondly, the demands and rules enforced by donors are complicated and sometimes impossible to comply with. An example is demanding a survey 
conducted during the rainy season to include rural schools to be part of the sample, when these schools are impossible to reach due to the road conditions. Therefore, Altner (1999) proposes to measure outcomes against the initial situation and by degree of national appropriation instead of international quality standards.

Thirdly, international organisations tend to rely heavily on experts from their own countries, who sometimes have too strong a voice in the implementation of activities and are not bound by their contracts to transfer their skills to their local counterparts. These international experts tend to work under time-pressure and provide input to Ministries without proper understanding of the Cambodian context (Altner, 1999; Beynon et al., 2004; Godfrey, 2000; McNamara, 1999; Siddiqui, Strickler \& Vinde, 2004).

One of Godfrey's interviewees, formerly working as an education management planner, described the difficulties of coordination between foreign donors at an agricultural institute as follows:

'Probably the largest difficulty of all was not inside Cambodia at all, but were imposed foreign time frames, foreign policy imperatives, foreign decisions about how money was to be spent and when, foreign decisions about who was to run programs...this was a source of great difficulty, and often local people seemed to find it hard to understand these constraints' (Godfrey, 2000, p. 89).

Another interviewee stresses that NGOs should

'... critically consider whether they are making an actual contribution and eliminate parallel projects. They (the NGOs) too are responsible for the situation in the country - not just the Government' (Verkoren, 2005, p. 301).

The demand for evaluation, monitoring and reporting by foreign agencies is often uncoordinated and placed on the shoulders of a limited number of competent local staff, pulling these people away from their other responsibilities (Siddiqui, Strickler \& Vinde, 2004).

Cambodianisation, also called 'Khmerisation' of staff is the replacement of international staff by Cambodian nationals, and the use of local NGOs to work with the Government instead of international ones. The idea behind such nationalisation processes is that Cambodia needs to take more responsibility for the direction of development and accelerate the capacity development of the 
country. Verkoren (2005), however, warns against this process. In her opinion, such a process could lead to delayed implementation of projects run by Cambodia's aid sector, thus threaten capacity building and their sustainability and in the worst case, even to stronger politicization. It could lead to, but it is more likely to cause further abuse of aid resources and increased nepotism. The Government should first raise the trust and confidence of its citizens in its institutions.

Beynon et al. (2004), and Siddiqui et al. (2004) call for a high-level institute for capacity development. Beynon proposes his own institute to be the one to open a branch in Cambodia, while Siddiqui calls for civil training institutions. It is difficult to see how separate institutions could develop contextualised projects to build the capacity of Government staff on-the-job and not contribute to the 'brain-drain' of Government staff to such institutions, if they are placed outside the Government and not within. Godfrey (2000) suggests that capacity development should be done from the inside out, with people placed inside the Government, to guarantee more success.

Another issue is the overwhelming amount of foreign aid workers in Cambodia mentioned earlier. The presence of many expatriates influences trade flows, employment patterns, wage structures and exchange rates. Their consumption, spending patterns and other behaviour, have an influence on local habits and culture that can be negative. On the other hand, it might have a positive influence when expatriates set a good example by promoting non-discriminatory behaviour in ethnicity, class, gender, etc. (Junne \& Verkoren, 2005b).

A final finding of interest, after analysing the aforementioned documents, is that international donors see sustainability as strengthening knowledge and skills of local staff, whereas the Government and local NGOs approach the sustainability issue almost completely from a financial point of view. For example, improving teachers' knowledge in technical areas that would remain after the termination of a project and promoting English language skills was mentioned as a sustainable project by international organisations. Even financial sustainability is seen as a chance for capacity building: developing production bases and teachers' capacities to run short courses that would generate income. The local organisations on the other hand, give examples like directing external assistance 
in production bases that could generate income, improving efficiency of production, trying to sell schools' services, exploring possibilities of running private courses and charging school fees. This difference in definition and operationalisation adds to the gap between what is said and what is done to guarantee sustainable projects. Godfrey (2000) concludes that, as a result, both Government and aid organisations make a token gesture towards sustainability.

\subsubsection{Professional development in practice}

Cambodians tend to value formal training courses, both in country and overseas. Study tours are made on a regular basis, but are seen as less effective. One ADB staff member described study tours as 'a waste of time' (Beynon et al., 2004; Godfrey, 2000). Research conducted by Godfrey (2000) shows that on-the-job training is the main method for professional development, with $92 \%$ of the projects investigated naming this method as a way of building capacity, but it remains unclear how on-the-job training is defined. There is no description of how on-the-job training is carried out and how it contributes to skills and knowledge acquisition. The conclusions are based on the assessments of the technical assistants involved and the counterparts themselves. Long-term staff retention after training is $48 \%$ and knowledge and skills acquisition is between 60 and $100 \%$. It must be noted that these figures are based on technical assistants' and counterparts' own perceptions and are not independently measured. Some findings of Godfrey's (2000) research are of interest however.

For instance, lack of human resources, weak political will, low quality of advisers and intricate donors' procedures are specified as having a negative influence on the quality of capacity building projects. Furthermore, high-ranking officials seem to think in the short-term and demand that foreign technical assistants carry out the tasks at hand, instead of building the capacity of Ministry staff. This leads to low ownership of projects by Cambodian staff. Getting people to work together and share knowledge and skills is difficult. Only a very small number of international experts are able to speak Khmer. Capacity development activities should be interactive and identify ways to teach people how to put into practice what they have learned. Acquired knowledge and skills should be reported and disseminated within the organisation after learning endeavours. One official

referred to the importance of informal activities outside of work for 'fostering trust and team spirit' (Godfrey, 2000, p. 47). 
Under-funding remains one of the main issues of the Cambodian civil service. It is one of the causes of low salaries, which itself is the reason why staff members need to do additional jobs during working hours. It also affects job promotions and access to training opportunities. A former technical assistant at an agricultural institute recalls that

'...the problems were the political dimensions of deciding who was going to be trained... there seemed to be a very clear sense that the main purpose of getting trained was to get a qualification to gain other employment, or to gain a trip out of Cambodia... there was little sense of training leading to a teaching job at the college...' (Godfrey, 2000, p. 85).

\subsubsection{Training and workshops in practice}

From March 2006 to August 2007, a variety of training courses and workshops were observed within MoEYS by the researcher. About half of the endeavours were conducted by Cambodian Ministry staff, the other half by foreign consultants. There was no clear difference between endeavours that were led by foreigners and those conducted by local trainers. Apart from one workshop conducted by the Early Childhood Education Department in Oddar Meanchey province, one by the School Health Department in Phnom Penh Municipality and one by the Pedagogical Research Department in the capital, all the events were organized in the same way, with lectures and whole-class teaching. Participants were seated in rows and were lectured. Depending on the length of the endeavour, one or more lecturers were responsible for the presentations. Sometimes an 'organiser' (in some cases this person was called 'facilitator' confusing the role with that of the facilitator of the learning process) was solely responsible for writing the flipcharts and carrying out all different kinds of supporting tasks. Different people usually conducted different sessions and facilitators only tended to come to conduct their own session. It was not common for trainers to attend other sessions as participants, although there were some exceptions. Opening officials rarely stayed beyond their ceremonial duties.

Trainings usually started with an opening ceremony around $8 \mathrm{am}$, by at least one high-ranking Government official, one or more representatives of aid organisations (if applicable) and a moment for the Cambodian National Anthem. After that, depending on the facilities of the location, PowerPoint presentations 
or flip charts were used for the lectures that were only interrupted for a coffee break around $9.30 \mathrm{am}$, lunch from 11.30 to 1.30 and an afternoon break around 3 pm. A typical training day ended around 4.30 or 5 pm. Snacks and drinks were usually provided during breaks, and sometimes lunch. Most people preferred to rest after lunch. Icebreakers and energizers were sometimes conducted after breaks, usually in the form of a short funny anecdote or song, presented by either the facilitator or a participant.

Although sometimes handouts were provided, participants tended to make many notes. Most participants seemed to be highly involved during the lectures. Participants usually asked questions only after a lecture, if any, and were often motivated by the facilitator to do so. During the lecture, many participants did not show any sign of non-comprehension. Instead, listeners sometimes nodded or smiled to the lecturer. Foreign lecturers sometimes mistook such signals as a sign of understanding and encouragement to continue lecturing. On the other hand, Khmer lecturers knew it could also be a sign of incomprehension. This is illustrated by the following example from a workshop on how to write a capacity building plan in March 2006, at the Directorate General of Sports, of MoEYS, which was typically organised:

'Approximately 25 participants were sitting in rows whilst the method for developing a capacity building plan was explained. Throughout the teaching, people were continuously nodding and smiling in a friendly way. This was interpreted by the international trainer as a sign of comprehension, and provided motivation to go beyond the original teaching intention. After the lecture had finished, during the break, some of the participants came forward one by one, asking for additional explanations. Some of them were the most ardent listeners. Their nodding and smiling turned out to have been encouragements to explain more clearly in the hope that they might understand what was meant, rather than being a stimulus to provide additional information, which had only complicated comprehension further' (Personal notes, 25 March, 2006).

This short anecdote also shows the tendency to postpone questions until after the end of the session. Questions are then asked on a one-to-one basis. Only minor questions, if any, are asked before the end of the lecture, during the time reserved for it. 
It was not customary for lecturers and other facilitators to evaluate their individual sessions, or the overall training, at the end of each day or the entire endeavour. This was partly because of the fact that so many facilitators only joined for their own particular session.

Facilitators or participants with decision-making authority were often interrupted during sessions by mobile phones, which they answered, or to sign documents brought in by staff of their offices. Sometimes pre- and post-tests were conducted, as were evaluations. What happened with the results of these activities differed. In general, the outcomes were not used as feedback to the participants. In the two instances that follow-up did take place, it was delivered in a 'top-down' manner, through lectures, instead of being collaborative problem-solving and learning activities.

Three exceptions to the 'lecture-style' courses described above were each different in another aspect:

1. A training session provided by the Early Childhood Education Department consisted of roughly 50\% learner-based and participatory activities, including icebreakers, energizers and evaluations. The energizers were greatly appreciated by the participants and worked well to get the participants motivated to work on the more substantive sessions. The remaining time was in the form of regular lecturing, using flipcharts that were written by an 'organiser'. In practice it meant that the organizer was writing flipcharts for five days in a row. During the interview with the Ministry staff responsible, it was explained that the course was developed together with a UNICEF consultant, who introduced the participatory approach and activities to the Department. They kept inflexibly to the initial activity schedule. The staff appreciated the participatory approach and asked the researcher for additional activities they could do with the participants. The important finding here is that, although participatory techniques are used, adaptability is not adopted. This leads to precise execution of what has been learned, instead of adapting it to other learning situations and creating new activities.

2. A workshop on Avian Influenza delivered by the School Health Department was a collaborative result from the work of three Ministries: MoEYS, the Ministry of Health and the Ministry of Agriculture. This is in itself remarkable, as for the first time a workshop was not the responsibility of just one Ministry. The persons responsible had divided the content for 
presentation. Thus, although the workshop was delivered in the usual way, with the lecturer teaching the participants seated in front of him/her, it consisted of a number of lectures that showed the different perspectives of health, education and agriculture, but also involved much repetition.

3. The third exception to the typical way of running training and workshops was a five-day workshop in 2006 aimed at developing the 'STEPSAM' science curriculum for secondary education (Japan International Cooperation Agency [JICA], 2008), held at the Pedagogical Research Department in Phnom Penh. Secondary physics, chemistry and biology teachers from a multitude of secondary schools were involved in this curriculum-writing workshop under the supervision of four Japanese university professors. Participants worked in groups that were mentored by Cambodian counterparts of the professors. The curriculum was the result of a collaborative process, and was presented at the end of the last day. Although the result was impressive, questions can be raised as to the applicability of the curriculum. When experts are invited to work together, sometimes the outcomes are very high-level, but difficult to implement. This was observed to be the case for the science curriculum as well. The Japanese experts came with examples that were probably applicable to Japanese secondary education, but hardly possible in Cambodia, where labs are not readily available and equipment is lacking.

These examples show that collaboration, shared responsibility and use of participatory approaches are possible in Cambodia. Such examples, however, are limited and in the early stages of experimentation. The facilitators who were observed by the researcher to experiment with these approaches were enthusiastic and expressed their interest in gaining more background to develop their facilitation skills.

\subsection{THE NEED FOR CHANGE}

Post-conflict societies tend to have a rent-seeking economy (Junne \& Verkoren, 2005b). In rent-seeking economies, some individuals, firms and organisations seek to make money by manipulating economic and legal systems. Such societies are characterized by low levels of education, lack of emancipation, high population growth rates, questionable freedom of the press, bad health conditions and a weak civil society. This applies to Cambodia, while striving to become a member of the 
globalised community. If Cambodia's ambitions are serious, then it needs to modernize its services to its citizens. Good education is the basis for any development, but can only contribute if trained people are offered jobs that fit their training. Cambodia committed itself to the 'Education For All' millennium goal and is trying to make an effort in strengthening its civil services. Developing the capacity of civil servants is an essential step in this process.

The Directors of the Basic Education Departments and DGE acknowledged that professional development activities needed to be improved. Interviews with five of the eight Directors, held in February 2007, revealed that only one of the Departments had a guideline for organising PDAs, and that this guideline needed to be revised. All Directors stressed the importance of having a set of guidelines to standardize procedures like monitoring and evaluation, and the improvement of facilitation skills of their staff. For the strengthening of the facilitation skills, they wanted the guideline to contain: 1) Methodologies for participatory and learner-based activities; and 2) Instructions to strengthen additional facilitator skills, for example, how to identify learning needs, how to run facilitator meetings, and how to keep discipline.

However, a guideline should not be followed inflexibly, according to one of the Directors: 'If my staff is smart, they do not follow any guideline rigidly, because they need to adjust to the situation in their provinces and workshops.' Another Director stressed the need for extending the facilitator skills for working with adults: 'Adult learning is different than training other people... our staff needs to learn how to train adult learners'.

According to the Directors, the most common activities used in training courses were instruction, discussion, presentation of group work, and Q\&A sessions. They expressed their preference for small group discussions instead of plenary discussions: 'In the big group only a few smart ones speak', although they felt that 'discussion in groups or plenary depends on size and time'.

Although all Directors seemed quite satisfied with the level of the professional development activities provided to and by their staff, they did see the need for improvement: '(Training should be) well planned, interesting and on important topics, it should solve specific professional issues, also for the future'. Regarding the preparation, a Director said: 'Trainers should discuss and share in advance, 
we should train our staff to do this more' and 'preparation on the content should be good, we need to know more clearly about the content ourselves.' To make sure the level and learning needs of the participants are known, 'needs analysis at participant level should be done'. Most Directors mentioned the wish to see PDAs become more learner-based.

What they also found very important was the motivation of the participants: 'If staff wants to do training, they are motivated and will perform well. If not, effectiveness will be poor.' All Directors wished to 'only train the people who need it', but they also said that this would not be easy to achieve, as staff use the 'per diem' payments that are provided for training to supplement their salaries. According to one Director, there is also an important role for the higher cadre: 'It also depends on the Director: if importance is stressed and shared, it will be more positive.'

The majority of the Directors said that trainers and facilitators are necessary for professional development. Although they said that the role of a facilitator is to support the trainer, and in some cases even to correct the trainer, they also said that they could not distinguish between the roles: 'It is not really clear what the difference is', and 'now we are unclear on the roles for the two, so we need to be more clear.' A similar lack of clarity was found to exist for monitoring, evaluation and follow-up activities. The majority of the Directors used the terms inappropriately, or were unable to distinguish between the different processes. They said 'all are forms of evaluation', or that 'follow-up is evaluation'. In addition, more than half of the Departments did not have formats and standard procedures for these activities. It is perhaps not surprising therefore, that all the Directors said they wanted the guideline to contain tools for monitoring and evaluation.

\subsection{IMPLICATIONS FOR A MORE EFFECTIVE PROFESSIONAL DEVELOPMENT SCENARIO}

Based on his research on Government staff, Godfrey (2000) defined quality requirements for technical assistance for capacity development. The Government should be involved in the selection of experts, and look not only for technical and regional experience, but also according to social qualities that are needed to develop capacity of staff. The weight should be on facilitator, trainer or 
communicator roles, instead of only managerial ones. According to Godfrey (2000), preference should be for long-term commitment instead of short-term support, because it takes time to build trust and understand the context, sometimes including fundamental social and cultural issues that need to be understood for effective implementation.

Cambodian counterparts should have well-developed English language skills and receive appropriate salaries or salary supplementations in order to be dedicated members of capacity development projects. Although Godfrey's report suggests that counterparts are only committed to projects when they receive sufficient financial compensation, at least one international expert described Cambodian counterparts as 'enthusiastic, not so much money-oriented and having conviction to do the work' (p. 38).

Projects should be based inside the Government, not in separate offices, as establishing outside project implementation units (PIUs) was seen as a barrier to capacity development. Post-project sustainability plans should be developed in order to guarantee continuity of institutional capacity development.

Simplifying the content of PDAs has previously been described for the UNESCO/UNDP project (Altner, 1999) and for other PDAs (Beynon et al., 2004). Usually, such simplification was done after it was found that the content of a course was too difficult for the participants. After simplifying the modules, understanding increased, but understanding as a whole remained superficial and did not lead to significantly improved workplace procedures. This was because of the first finding of Beynon et al. (2004): the lack of application possibilities because the modules were too theoretical. Evaluation of the UNESCO/UNDP training called for more interactive participatory training techniques, improved trainers' practical understanding of the content, ways of strengthening work practice and acquisition of skills, and a more systemic approach to professional development. Beynon et al. (2004) concluded that, at least until 2004, nothing had been done with these recommendations. The findings presented in the next paragraph lead to a similar conclusion.

Similar findings as those of Beynon et al. (2004) are reported in a training needs analysis for the Cambodian health system (Domrei, 2006). According to this analysis, PDAs should be much more practical instead of theoretical, the limited level of comprehension by trainees should be respected, different concepts and practices for training adopted, trainers trained, real-life stories and materials 
used, simple guidelines for the preparation of training sessions developed and training followed-up.

Although strengthening the skills of trainers is - explicitly or implicitly mentioned by all researchers, it is not explicit that such strengthening should include methodologies and training skills. Analysis of a training needs assessment conducted for MoEYS in 2006 to develop training for writing successful granting proposals (Tat, 2006), is that course designers chose the most appropriate training methodologies based on the content of the course, not on their suitability for the context. The outcomes of the needs assessment reflected that, but also perceived the trainees as tabulae rasae: it was proposed that the course started with a presentation of theories, followed by discussions on case studies, and ended with feedback on the outputs from those discussions. Previous experiences and training were not taken into account.

Training of Trainer (ToT) programs in Cambodia too often focus on theory instead of practice, probably based on the over-appreciation of theory over practice (Beynon et al., 2004). Particularly at higher levels a theoretical approach is appreciated. It might even be that a participatory, more learner-based approach is seen as something for children and less educated people.

One interviewee in Siddiqui's (2004) research raised his concerns about the soonto retire top of the Cambodian civil service. These people are educated and experienced, whereas their younger staff is much less experienced and, in some cases, less educated. This group of soon-to-be leaders should be prepared for their future jobs. Both the existing and future knowledge gaps are a major constraint towards achieving sustained development and implementation of needed reforms (Siddiqui, Strickler \& Vinde, 2004).

In conclusion, many factors influence professional development and continued strengthening of capacity of Cambodia's civil service remains necessary. Training is seen as an important method, but how training courses should look like is only marginally explored by the surveys, needs analyses and reports. Every document stresses the importance of training, but the characteristics of effective training in Cambodia remain ill-defined, although the need to provide less theory and more practice is sometimes mentioned. 
One issue that the Ministry of Education has tried to work on is the improved coordination of professional development. With the support of UNICEF, the Ministry installed a Capacity Building Coordination Group (CBCG) in the DGE in 2001 (Chea, 2005). The CBCG is an active body and meets every month. Each Department under the DGE is represented by a Deputy Director. The task of the group is to coordinate PDAs and, over time, develop a multi-year capacity building plan (MoEYS, 2005a). That plan was established in 2007, for the period 2008-2010 (Berkvens, 2007a). This capacity building plan was a first attempt to streamline capacity building into a planned approach.

One of the outcomes of the multi-year capacity building plan developed was the establishment of a working group whose task it was to develop a Training Guideline for all Departments under the DGE. This process was initiated and supported by the Deputy Director General of Education, who wanted the Training Guideline to contribute to a more coherent approach to professional development activities while using more participatory formats.

The Ministry of Education has to play a major role in restructuring education and therefore has to upgrade its own capacity. One important factor in such upgrading is the skills of its staff to be facilitators. Knowledge about professional development and adult learning is essential. The process through which to arrive at the Ministerial Training Guideline is the object of this research. The implications of the context analysis presented in this chapter are summarized below.

The exploration of the context described in this research, leads to the following design principles for the scenario:

- Allow for enough time to build trust. Trust is essential for effective learning in Cambodia, but has not been given enough consideration in the past.

- Understand how power distance influences professional development activities, as power issues are abundantly present in Cambodia and do influence the degree to which people feel safe to share information and work together collaboratively.

- Ensure the full participation of staff, regardless of their hierarchical position and create a safe working environment to support that.

- Ensure that responsible senior staff members are informed about the objectives, progress and outcomes of the scenario. Experience shows that senior staff's understanding of, and involvement in, PDAs boosts support and reinforces outcomes. 
- Ensure that all Departments of the Ministry are involved in the scenario, in order to arrive at broadly valid and usable outcomes.

- Extend learners' methodological understanding and repertoire, and strengthen their facilitation skills, in order to enable them to become master facilitators.

- Allow for enough time for people to join in meetings and ask questions outside the meetings, by being available as much as possible.

- Use each meeting or interview as an opportunity for acquiring a better understanding of the Cambodian and working culture. 


\section{CHAPTER 4}

\section{Literature review}

\subsection{INTRODUCTION}

The previous chapter analyzed the context in which this research took place. Based on the findings, context-specific guidelines were identified. This chapter examines the relevant academic background on human resource management (HRM), professional development (PD), curriculum development, adult learning and culturally relevant learning, and applies these theories to identify design principles for the scenario described in this research in order to strengthen effective professional development. The fields of interest explored in this chapter have been selected in order to embed 'adult and effective learning' into the bigger processes of organisations. Learning endeavours, like training and workshops, in organisations are not isolated events. They act together in a system of career possibilities and rewards, but are also influenced by cultural issues. HRM, for example, is of interest to this research because many of the HRM models address personnel management including training and learning activities, career opportunities and rewards, but has different approaches, of which some are compatible with the Cambodian context, and others are not. Power issues and hierarchy are important aspects to take into account. PD encompasses the learning component that is crucial in the broader picture of HRM. Empowering people to learn is a key concept in PD, but challenges Cambodian cultural norms. How curricula are developed is of interest, because a curriculum shapes the learning that occurs in professional development programs. It can either be prepared in advance, or developed with the learners during a learning program. The exploration of the latter provides relevant insights into how the experience of Ministry staff can be used in order to learn collaboratively. Such learning is further explored in the field of adult learning. The notions presented with regard to culturally relevant learning provide suggestions to ensure that the learning fits 
the cultural context. This chapter will show that almost all academic theory presented in this chapter is Western based and needs to be contextualised before it is introduced to different contexts. A number of examples will show the effects of ignoring such contextualisation.

Prior to any discussion on the theories of adult education, two points need to be made. The first relates to the importance of being aware of the differences between formal, non-formal and informal learning. Formal learning takes place in learning institutions, such as schools and universities, whereas non-formal learning consists of training programs in companies and organisations. Both follow a certain curriculum, whereas 'informal learning' does not. The view that formal learning takes place 'off-the-job' and involves theoretical learning, and non-formal learning is 'on-the-job' and is mainly for practising application of what has been learned is outdated (van der Sanden, 2001). Non-formal learning complements formal learning in multiple ways: It is usually less structured and more flexible than formal programs and has been described as being more spontaneous learning in normal daily life, or as 'everyday learning' (Coombs, 1985; Illeris, 2004; Merriam, Caffarella \& Baumgartner, 2007). Perhaps because of these features, it is a common format for international development. These programmes are usually financed by non-governmental organisations (NGOs), donor or private organisations and are mostly concerned with solving social inequities or raising consciousness towards social action. Informal learning is the most common form of learning in the workplace and occurs through informal interactions with others (Dirkx, 1999; Marsick \& Watkins, 1997; Skule, 2004). Non-formal and informal learning processes in the workplace are often more powerful than those in formal training institutions (Kessels, 1993), as formal education is usually too far removed from the reality of daily work. The learning discussed in this thesis is mainly non-formal.

A second important comment is that the existing academic knowledge base discussed in this chapter is Western-based and Western-focused. The chapter includes examples of Western arrogance and 'unconscious ignorance' found in some of the literature describing professional development projects in developing countries. It is important to be aware of such latent beliefs, as they might have caused bias in the conclusions arrived at in the literature being explored. For example, Wright (1997, p. 383), at that time being a consultant, describes the Mayor of the African town he works in: 'Each time the facilitator met with the Mayor, who was very bright and tended to ramble...' 
The implicit belief behind this description is obvious: It is worth mentioning that the Mayor of this African town is bright, but he is still not completely competent, as he tends to 'ramble'. Such beliefs about capability of local staff and leaders are subjective and judgmental. They are also unconsciously arrogant and might well influence how foreign experts work within organisations in developing countries.

Examples of how beliefs held by foreign experts influence the way they interpret the situations in which they work are rife, both in Cambodia and in general literature. It is important to understand that authors are often unaware of their own (cultural) biases and assumptions.

\subsection{HumAN RESOURCE MANAGEMENT}

\subsubsection{HRM in developing countries}

Human Resource Management (HRM) is in its infancy in developing countries. HRM encompasses Human Resource Development and the older notion of Personnel Management and stresses the need for a systemic approach. Most developing countries, and especially their public sectors, use hierarchical forms of personnel management, rather than following more modern HRM theories. These approaches make it unlikely that these organisations are utilizing their human resources effectively (Budhwar \& Debrah, 2001), as staff is less empowered and able to take responsibility.

An integrated approach would combine different components like training programs, career opportunities and more competitive salaries, in order to empower staff. The move towards such integrated and less hierarchical HRM systems and institutional reform in developing countries is mainly promoted by foreign agencies (Budhwar \& Debrah, 2001; Grindle, 1997). Some authors claim that assistance by foreign agencies usually involves extensive investment, but delivers little increased capacity (Berg, 1993; Jaycox, 1993). Others are more positive about the effects, but stress the importance of good project design and contextual understanding to be successful (Grindle, 1997). Budhwar and Debrah (2001) identified three important domains for such understanding: national culture, national institutions, and the dynamics of the broader environment. Failing to understand or misunderstanding local HRM practices could lead to the 
imposition of systems on organisations that do not fit the context and hence will not be successful. For instance, Werner (2001) gives some examples from Africa that, according to him, show that traditional cultural beliefs sometimes compromise effectiveness by ignoring key concepts of HRM like integrity and efficiency and following local customs giving precedence to socio-cultural relations instead. He also describes the importance of keeping harmonious interpersonal relations intact in China. Different approaches in HRM are demanded where the prevention of 'loss of face' and the following of strict hierarchic relations are important. Peterson (1997) concluded that it is better to build on 'what is' than on 'what ought to be' in the eyes of Western organisations.

Werner's examples show clearly that relationships are believed to be more important in some cultures than, for example, efficiency which is a key notion in Western organisations. It is thus important to identify local cultural and organisational customs and not impose a system with embedded beliefs and concepts that do not fit the particular context. Furthermore, learning could imply development that might include (organisational) cultural change. The difficulty is how to identify when change is genuinely and intrinsically desired, and when change is mainly cosmetic to satisfy foreign involvement. Van Eijbergen (2005, p. 12) describes a situation in Uzbekistan in which an applicant was rejected based on his sexual orientation, but eventually hired after a discussion between van Eijbergen and his colleagues. Van Eijbergen described this as a positive development, but one could also argue that he forced his Western conviction on his colleagues. He also assumed that the discussion changed the cultural beliefs of his colleagues. Such assumptions are, at best, arrogant, and could easily turn into patronizing behaviour.

\subsubsection{The need for a more integral approach of HRM}

The South African practice of HRM shows that a more integrated approach can work in developing countries. After four decades of apartheid and following affirmative action programs in South Africa, large groups of under-skilled staff entered public services, increasing the demand for training. Wood and Mellahi (2001) found a subsequent need in South Africa for a coherent qualifications framework recognizing both formal and non-formal education and a need for policies to encourage institutions to take training more seriously. The South African Employment Equity Act and the Skills Development Act are 
complementary to each other and create opportunities for skills development and workplace learning (Groener, 2006).

The South African findings are significant for the Cambodian context, as the civil services of both countries have a similar need to train staff both formally (courses in institutions) and non-formally (training programs at work). Countries like South Africa and Cambodia, with scarce or inappropriate formal professional training, compensate for this situation with non-formal in-house training programs at or alongside the workplace (Hilderbrand \& Grindle, 1997). Examples are the ten-day training program on how to write curricula, or a course on how to teach life skills at primary schools, both offered by the Ministry of Education in Cambodia.

Whether professional development is established through formal or non-formal routes, the outcomes should be complemented by a recognised reward system. Such a system is part of an integrated approach of HRM. Cohen and Wheeler (1997) indicate that such HRM structures (also in developing countries) include career prospects with increasing responsibilities and income, and encourage greater productivity. The Cambodian Government made a start at working towards a more systemic approach with the production of the 'Staff Development, Accreditation and Reward Systems' report in 2006 (EFC, Indochina Research \& Span Consultants, 2006). The report describes a complete system involving career patterns, competitive salaries, evaluation files and personal 'professional development planning forms'. It remains unclear how 'contextualised' this proposal was and whether the Government adopted it, since the document follows a contemporary Western HRM structure. It is highly unlikely that the existing organizational and national culture with its specific power structures and norms can easily be changed to make implementation of such an ambitious (and Western-style) plan successful. The African and Chinese examples presented by Werner do not allow for high expectations of this.

\subsubsection{Power issues in local HRM systems}

Local power structures are of significant influence to any change project, and thus for the implementation of more systemic HRM systems. Any official staff bearing relevant responsibility for a certain project should be involved, or at least kept informed, in decision-making processes, in order to reduce the chance of dismissal of any ideas generated (van de Poll, 2005; Wright, 1997). Malvicini 
(2006) argues that changing towards more contemporary HRM systems might threaten the power of leaders. He found that open, inclusive dialogue involving not only learners and managers, but also organisational leaders with greater power, is less threatening. Such a strategy probably works if it does not devalue the powerful position people hold and keeps them involved in the decisionmaking process. An issue that could arise is that lower-ranking staff members might be unable or unwilling to share their opinions openly. Another issue is that in Cambodia, and presumably in many developing countries, there is an extensive layer of higher-ranking officials in Government organisations. This leads to the question as to who should or should not be invited in order for these dialogues to become or remain effective.

Authors describe different conditions for systemic approaches, including good working environments, proper incentives, inclusion of powerful people, possibilities for utilization of acquired knowledge and post-training skills (Hilderbrand \& Grindle, 1997; Grindle, 1997). In addition, Kessels and Keursten (2001) argue that learning is a function of participation in meaningful work.

In many cases, however, possibilities for utilization are limited or absent, because senior management does not acknowledge the professional development of its staff and persists in the same hierarchical and old local customs without delegating responsibilities. Many trainees believe that their jobs are not meaningful and not suitable for effective employment of their skills at work. Providing staff with meaningful jobs leads to a higher retention rate of trained staff. There is an ongoing debate about the risk of brain-drain, staff turnover and low retention after the provision of training. Many authors mention the disastrous effects of providing people with more skills in settings where the rest of the spheres have not changed, as it leads to high staff turnover (Cohen \& Wheeler, 1997). Other authors, however, see benefits in high turnover, because it gives young highly educated staff the opportunity to take over from the older, more established staff (Contreras, 1997). The latter point of view is challenged, however, by the observation that in Cambodia it is usually the young and newly trained staff that leave the Government to work with other companies and organisations, thus not contributing at all to the renewal of the Government's human resource structure. 


\subsubsection{Support issues in local HRM systems}

Many consultants are actively organizing HRM structures in Cambodia and in developing countries all over the world. Discussing the roles, responsibilities and the influence of such support is important. Multiple terms are in use for the role of the advisor, like technical advisor, trainer or facilitator. Whilst authors might disagree about the correct term to be used, they all agree on the importance of advisors having an open mind and willingness to understand the context in which they work. As Wright (1997, p. 405) puts it:

'Donors (and the staff they hire) must genuinely understand, not superficially, that their perceptions of needs, goals, priorities, methods, procedures, practices, values and standards may be very different from those of the recipients with whom they are working.'

He calls this discrepancy between perceptions 'differences in frames of reference' that need to be overcome in order to be able to work effectively. More often than not, expatriates do not see the need for such collaboration or are pursuing shortterm, opportunistic agendas (Grindle, 1997). Such attitudes make it impossible to understand the context. This is a dangerous situation, because it reinforces already paternalistic and involuntarily arrogant values held by Western aid workers. As Bikales (1997, p. 456) puts it, based on his experiences in capacity building programs in Mongolia: 'Selecting proper advisors is essential. They should be rightly skilled and willing to share skills with the counterparts'.

Fasheh (in Merriam, Courtenay \& Cervero, 2006. p. 387) calls for a respectful attitude, requiring understanding, openness and humility from the side of the aid worker in order to develop mutually respectful relationships. He maintains that good intentions to assist and empower people are essentially disrespectful but have become normalized behaviour on the part of those who would be the helpers towards those who are to be helped. Learning to understand the context and supporting Governments and organisations to develop should become a collaborative learning endeavour between advisors and local counterparts.

Analyzing this, one could question the current wording used for the roles of expatriates and local staff. Maybe consultants should be called '(international) counterparts', emphasizing their supportive role, instead of the local colleagues being there to support the international consultant. 
Western arrogance surrounding the relationship between international advisors and local staff is abundant, as illustrated in the following description of the successfulness of a project in Pakistan: 'This ... was accomplished largely through the efforts of Tom and his Pakistani assistant' (Wright, 1997, p. 382).

In this example the Western expert has a name, but the name of the local counterpart is apparently not important. Furthermore the counterpart is addressed as an assistant to the Western consultant. It would be interesting to know who did all the negotiations and in what language. It might be that the way the Pakistani counterpart said or translated things safeguarded the accomplishments.

In some situations, Western arrogance is more subtle, as in the following categorical statement of van de Poll (2005, p. 22), who worked extensively as a consultant and project manager in HRD projects in the Middle East and Central and Eastern Europe:

\section{'Understand the culture in which you work, but do not conform to it.'}

One could argue that not conforming to deeply-rooted practices like corruption would be a positive thing. But it is important to understand that one makes such judgments based on the definitions of one's own background and the beliefs held within one's own culture. The point is that not being willing to conform to local culture and concepts automatically means that people of the local culture should conform to the culture and concepts of the other. In this example there is no questioning of the personal culture of the expert. It seems like van de Poll sees consultants as positive role models of how things should change, emphasizing Western superiority.

\subsubsection{Design principles from the HRM perspective}

Based on this analysis of HRM theory, the following design principles for the scenario were identified:

- Perform an exploration of the local and organisational culture, the existing power structures, and any influences from the surroundings on the Ministry organisation in order to develop a thorough understanding of the context. 
- Take 'where people are' as the starting point for the scenario, instead of 'what ought to be', in order to develop a scenario that is relevant for Ministry of Education, Youth and Sports (MoEYS) staff.

- Promote an integrated approach of HRM in order to include necessary (pre) conditions and opportunities for utilization. This has been attempted before, but remains necessary, as these preconditions shape a more positive environment for professional development.

- Involve leaders in the scenario development process in order to secure their support to implementation. If it is impossible to involve leaders on a continuous basis, then they should remain informed throughout the process.

- Create a safe learning environment in order to safeguard the input and learning of participants. Collaborative learning can only happen when people feel safe about sharing ideas. Fear is repeatedly mentioned as an obstacle for constructive learning in Cambodia.

- A respectful and open-minded approach must be adopted in which mutual learning is key. Too often external advisors have been providing input to MoEYS from their own (Western) points of view, leading to solutions that were not appropriate for the Cambodian context.

\subsection{PROFESSIONAL DEVELOPMENT}

\subsubsection{Professional development and learning}

The literature on HRM addresses many components that influence the effectiveness of professional development endeavours. However, the learning component itself, the part in which the actual learning should take place, is much less explored. Such ignorance is widespread in HRM literature and in articles on HRM in developing countries. Senior Government officials in Kenya argued (Cohen \& Wheeler, 1997, p. 132): 'So long as there is a net addition of trained personnel to the country's human resources, the training component is successful...'.

This naïve statement assumes that the learning component in itself is useful and effective. Although the training component is seen as an important part of any systemic approach, at the same time its design is taken for granted. The effectiveness of training is assumed to be in order, and indications for failure of 
capacity building projects are merely sought in organizational issues after training took place. There are few studies reviewing the successes and failures of training, and most of it is anecdotal evidence (Cohen \& Wheeler, 1997).

Professional development is an intentional, ongoing and systemic process to enhance professional knowledge, skills and attitudes (Guskey, 2000). Joyce and Showers (2002) present a model for acquisition and utilization of such knowledge skills and attitudes in the work situation. They identified four training components (study of theory, demonstrations or modelling, practice, and peer coaching) and calculated their singular and combined effects on knowledge and skills attainment and executive implementation - or high-level transfer. The results of their research shows that only a combination of peer coaching with all other components leads to a 95\% score of executive implementation. All other combinations do not have any meaningful effect on high level-transfer, i.e. application in the workplace.

Contemporary professional development or human resource development has moved away from the viewpoint that only individuals can learn. So can groups and enterprises. The notion of the learning organization has become widespread. In learning organisations it is the individuals who learn, but the sum of the individuals learning together in a group or an organization as a whole is more than just the learning effect of each of the individuals alone (Bolhuis \& Simons, 2001; Kessels, 1996; Tjepkema, 2001). Social learning processes within interdisciplinary teams or communities of learners can create new ideas and share them, leading to preservation of knowledge in an organisation, even if individual team members leave (Stewart, 1998).

The learning organization, with interdisciplinary teams or communities of learners, is ideally a knowledge-productive organization, in which relevant information is identified, interpreted and generated in order to apply this knowledge to improve work processes, products or services. The knowledge referred to here is situated knowledge, as it is constructed within a certain context. This context is continually changing, resulting in organizations needing to keep pace with the demands of modern society by improving and renewing their processes and products (Kessels \& Keursten, 2001). 
Learning together is imperative for a learning organization model, and involves knowledge acquisition in the broadest sense. Knowledge and skills acquisition is important to optimize problem-solving abilities and come to new, unusual and sometimes radical solutions. Meta-cognitive skills are important to reflect on the acquisition of knowledge and skills and how to employ them. Communicative skills are necessary to gain entrance to knowledge networks and to engage in innovative processes with others. Regulative skills are important to manage motivation, emotion and to remaining committed and involved in the learning processes (Kessels, 1996). It is clear that in such a model as the learning organization, working with others and being responsible for one's own metacognitive processes are key. Learning organisations should support their personnel in learning by providing supervision, mentoring, coaching, performance and assessment interviews that complement the strong learning environment of the organisation.

Interdisciplinary teams and communities of learners are just two of a multitude of perspectives for collective capacity building or strengthening. Lowyck (2001) developed categories of approaches for collaborative capacity building, of which two categories are important for this research. The psychological approach seeks to develop a common, shared language and arrive at shared views and values within a group of people. The system dynamic perspective believes that organisations learn through understanding the whole picture of powers and relations, in order to form a commonly shared working-system. Developing a shared set of definitions and approaches would be beneficial, as would a broader understanding of how decision-making processes occur within MoEYS.

\subsubsection{Empowering people to learn}

Learning together means empowering people by letting them participate in collaborative processes. Staff members must be empowered to construct knowledge. 'Knowledge workers' thus become equal partners who manage themselves and need autonomy (Drucker, 2000; Stewart, 1998). Therefore, managers should change from managing learning processes to supporting them. Leaders should demonstrate a learning-committed leadership style which is manifested by creating learning opportunities, serving as developers of learning, offering visible support and creating space for learning, encouraging risk-taking, promoting the importance of sharing knowledge and skills, giving positive 
feedback and serving as role-models (Ellinger, 2004). This means a radical but critical transformation for most managers, as they need to yield control over learning to their staff members (James, 2002).

In worst-case scenarios managers might block learning when they organize work and learning based on power, position, compliance and applying bureaucratic roles (Kessels \& Keursten, 2001). These managerial 'don'ts' describe precisely the current way of managing within MoEYS and many other civil organizations in Cambodia, thus blocking effective learning processes in these organisations. Micromanaging, telling people what to do and unsupportive managers are negative contextual factors found in many organisations (Ellinger, 2004) and are not unfamiliar to the Cambodian setting where technical expertise is limited. A necessary power shift as described above is necessary but highly unlikely to happen soon. Encouraging risk-taking, promoting sharing, giving feedback and serving as role-models are not widespread occurrences within the current local and organisational culture. Weggeman (in Rondeel \& Wagenaar, 2001, p. 30) describes these required change processes in hierarchy in the following way: 'An organization can no longer be led on the principle 'the top thinks, the floor conducts'. He further postulates that managers need to have trust in the knowledge of their staff members, as well as in their loyalty to the organisation to use their knowledge for improving the organization processes and outputs. Trust, as was explained in Chapter Three, is a difficult issue in Cambodia.

In order to support learning, the role of the educator is modified to that of facilitator, or catalyst. In the past, some catalysts of learning were too radical, leading to a disturbance of peace in organisations. In many settings, staff members try to work more subtly towards change, minimizing negative personal outcomes (Malvicini, 2006). It is important to remember that the role of educator shifts towards the role of learning facilitator. Instead of designing a sound learning route, a facilitator should be more of a coach for reflection and learning (Poell \& van der Krogt, 1999). Heron (1999) described an intricate system for classifying facilitation levels of learning facilitators, combining six learning dimensions with three facilitator modes. The learning dimensions say something about learning orientation (goal oriented, cognition oriented etc.), the facilitator modes about the level of directedness (hierarchical, cooperative, autonomous). Although the system is too complex and detailed to be of use itself in the research described in this dissertation, it informs the development of a more usable typology of facilitators' levels, which is presented in Chapter Seven. 
Whether a change towards the support of learning occurs or not, there is a risk of creating a new inequitable class-system, since less educated or lower achieving staff members are usually excluded from learning processes to develop their competencies in favour of high achievers (Kessels \& Keursten, 2001). In Cambodia such a system based on inequality already exists, but is merely founded in political preference and nepotism. Similar examples are found in Egypt, where family connections favour promotion (van de Poll, 2005). It is a Western assumption that a meritocratic competency structure could easily replace such nepotistic systems.

Empowering people to enrol in collaborative learning and decision-making activities involves the participation of people. Some authors, however, are critical towards participatory approaches and warn against their inappropriate use (Cooke \& Kothari, 2001; Malvicini, 2006; Wright, 1997). It is argued that participation becomes more of a tyranny or a habit instead of a way of empowering people. Without recipients taking ownership, self-reliance and responsibility will remain utopian ideals. Malvicini (2006) argues that participation in development work must be nuanced; otherwise it will perpetuate dependence and undermine the selfreliance that it claims to support. Although Malvicini based his findings on community empowerment endeavours in developing countries, it is of importance for participation at other levels in these countries as well.

A Cambodian example of a missed opportunity to empower staff is the policy presentation regarding an ICT project for schools in Cambodia at a consultative meeting at MoEYS that was conducted in 2006. A foreign consultant presented the approach, seated behind his computer, while his Cambodian counterparts were sitting behind him. They were introduced as 'his counterparts', but were not introduced further. The rest of the inner circle was filled with foreign representatives of funding agencies and supporting organisations, while the Cambodian counterparts composed the outer circle. The discussion was a bickering between the foreign representatives. The seating arrangement of this meeting was not pre-arranged, which makes the observation even more worrying, as it could indicate an unconsciously established pattern.

The freedom to be responsible for one's own learning process or that of a group might prove to be challenging in a hierarchical setting such as that found in Cambodian Ministries, especially with the high demand and control required by international donors and NGOs. Furthermore, the ideal state-of-the-art learning 
assumes individuals to have strong reflective, meta-cognitive and regulative skills, and organisations to know how to work with staff possessing such skills. It should not be assumed that such high-level skills are inherent in individuals and organisations everywhere, and certainly not in developing countries like Cambodia at this point in its development.

\subsubsection{Design principles from the Professional Development perspective}

The analysis of professional development theory leads to the following design principles for the scenario:

- An effective community of learners should be established by forming a wellconsidered interdisciplinary team. Such a team should aim at identifying and interpreting relevant information, in order to apply this knowledge and improve work processes, products and services. Working collaboratively is not common in MoEYS, making it impossible to share knowledge and skills and learn effectively.

- Ensure that all components of the effective training components model (Joyce \& Showers, 2002) (acquisition of theory, modelling, practice and peer followup) are included in the scenario, in order to optimize learning effect. Professional development trajectories in Cambodia rarely go beyond the first and second component.

- The scenario should aim to strengthen the meta-cognitive, regulative and communicative skills of Ministry staff, in order to enable them to join in more learner-based, collaborative and participatory learning activities. MoEYS wishes to adopt a more participatory learning approach in order to make professional learning more effective, but its staff is often too shy to participate in collaborative and participatory learning processes.

\subsection{CURRICULUM DEVELOPMENT}

\subsubsection{A consistent curriculum}

The briefest definition of 'curriculum' as being 'a plan for learning' is given by Taba (1962). The term 'curriculum' applies to all levels of the layered learning and teaching framework, from macro-level (the society and central ministry) to mesolevel (school), and of course micro-level (the classroom) (van den Akker, 1998). 
The curricular spider web of van den Akker (2003) has already been introduced in Chapter Two. The nine components forming the web are interconnected and linked to the rationale, forming a model for balance and coherence. The spider web analogy shows the vulnerability and need for coherence between the components, as 'any dramatic shift in balance will pull the entirety out of alignment' (McKenney, Nieveen \& van den Akker, 2006, p. 68).

Coherence between the different components as represented by the curricular spider web determines the internal consistency of a curriculum. On the other hand, external consistency is determined by homogeneous notions about the causes, needs and approaches to address problems (Kessels, 1993). Consensus between the people concerned reinforces external consistency. Managers who do not value or appreciate the knowledge and skills acquired by their staff members are indicators for low external consistency (Lowyck, 2001). Examples of low external consistency within the Cambodian civil sector are abundant, where many stakeholders have different needs and agendas and, in many cases, are driven towards meeting their own objectives without a real interest in coming to a more collective approach.

Contemporary curriculum designs move away from the narrow approach of the curriculum as a product to a curriculum as a continuous cyclic process involving multiple stakeholders and participants in a collaborative developing program of learning in order to reinforce fruitful development. One example is the corporate curriculum (Kessels, 1996).

\subsubsection{A corporate curriculum}

A corporate curriculum, notes Joseph Kessels (1996), is not an established curriculum or a crystallized set of courses, but a learning program developed by the learners of an organisation themselves: the learners, or groups of learners, decide what knowledge and skills they wish to acquire, and how they wish to acquire them, through constructivist dialogues on norms and values and what is needed to determine quality of work. According to Kessels (1996), a corporate curriculum should support learning with the following functions: a) Acquisition of content expertise and competencies; b) Learning to solve problems with that expertise; c) Developing reflective and meta-cognitive skills to find new ways of dealing with issues d) Acquisition of communicative and social skills for 
collaborative activities; e) Acquisition of regulatory skills; and f) Promotion of both stability and functional instability. The latter two functions need some further discussion, especially for the Cambodian context.

Kessels (1996) argues that stability is necessary in order to promote deepening of learning. On the other hand, a certain amount of functional instability is necessary to stimulate creativity and innovative thinking. Homan (2005) uses a mountain landscape as a metaphor to describe this idea. The stable state of group learning processes is in the valleys: people find consensus and tend to exclude other realities and opinions that might threaten the comfort of the stable state (compare with 'group think' later on in this thesis). This stable state, says Homan, is undesirable. The group of learners should try to get on the summit instead to reach a state of instability in order to develop. The further a group gets from the stable and safe environment of the valley, the more ambiguity will arise. This ambiguity raises the need to find new meaning and new solutions within the group of learners. Homan uses terms such as group-web to describe such a network of people that attribute meaning to processes and formulate solutions to processes. He argues that learning takes place when people are forced out of their safe and stable state and are forced to 'group-web' again. The role of a learning facilitator is to create conditions in which the chance to learn is the greatest, thus stimulating the group to reach for summits (Homan, 2005; Kessels, 1996). Such a learning or knowledge facilitator would be responsible for facilitating learning processes and creating strong learning environments (Rondeel \& Wagenaar, 2001).

Van Lakerveld (2005) considers a corporate curriculum as a learning environment in the workplace. In many Southeast Asian countries, such workplace learning is supported by Government policy to ensure its contribution towards nation building. In Singapore, for example, it happens in a wellorchestrated way, with the Government playing a strong leading role (Ashton, 2004). In Cambodia, workplace learning is implemented in a much less coordinated way (Berkvens, 2007a).

Van Lakerveld (2005) also highlights that a corporate curriculum is developed collaboratively, as a living or growing curriculum, based on the input of the people involved. The process of arriving at such a curriculum follows a 'learning by designing' approach (Lowyck, 2001). Both learning and designing processes turn into non-linear, cyclic and iterative endeavours, leading to an 'enacted 
curriculum' that becomes more and more appropriate to the situation. During the process of curriculum enactment, learners and educators act together in this process, becoming co-designers within their own community of learners in order to create cohesive training scenarios. Fasheh also mentioned such 'co-authorship' (in Merriam, Courtenay, \& Cervero, 2006). Design and implementation act together in order to achieve learning. What is gradually learned is then called the curriculum' (van den Akker, 1998).

The impact of a corporate curriculum following a learning-by-designing approach to professional development becomes visible if the endeavour is seen as a learning project. Usually a learning project is organised around a work issue, a problem or a relevant theme. The activities people execute within such a network of learning are explicitly to improve the work and learn from it (Poell, 1998). Thus the organisation is improved through the learning of the employees. Poell and van der Krogt (1999) identified four ideal types of learning projects. Vertical learning projects evince strong control by the educator and are determined by the line manager. Horizontal learning projects are less controlled and more supported by the educator and manager. An external learning project takes place outside the organisation itself, with the educator suggesting approaches and the manager providing facilities. A liberal learning project gives significant freedom to the learners, supported by both educator and manager. Most learning projects in Cambodia are external (in the sense that they are not embedded in daily work) and vertical, but the control over learning and support are usually weak.

\subsubsection{Design principles from the curriculum development perspective}

Analysis of curriculum development theory leads to the following design principles for the scenario:

- Ensure internal consistency of the scenario, by pursuing balance between the components as presented by the curricular spider web.

- Ensure external consistency of the scenario, by exploring and clearly articulating the needs and wishes of stakeholders. In Cambodia, expectations are often not clearly articulated and shared, leading to weaker impact.

- Adoption of a corporate curriculum approach, in order to enable the scenario to develop into 'what is needed', within the mandate provided by MoEYS. This approach also fits to the exploratory character and the learning-bydesigning approach and aims to lead to a balanced and fine-tuned scenario. 
- Create functional instability while developing the scenario, in order to stimulate creativity and innovative thinking. Learning takes place when learners are stimulated to leave their 'stable state', but fear should be prevented, as many Cambodian learners are sensitive to insecurity.

\subsection{AdUlt LeARNING}

\subsubsection{Characteristics of adult learning}

Professional development or capacity building in organisations automatically involves some kind of adult learning. Adult learning and lifelong learning are often presented as being interconnected.

According to Knowles (1990), adult learning differs from teaching children. Unlike children, he claims, adults know what they need to learn, feel that they themselves are responsible for their learning, have more experience that influences learning in both positive and negative ways, are ready to learn what they think they need to learn, have a desire for learning that is more life / task / problem centred and are more intrinsically motivated to learn, driven by forces like self-esteem, job satisfaction or quality of life.

Kearsly and Schneiderman (1999) confirm that adults have much more experience to rely on and that new skills and understanding are based on existing ones. They emphasize that certain methodologies like case studies, role-plays, simulations and self-evaluations are more appropriate for adult learners leading to meaningful learning. This leads to a shift from the content to the process of teaching. Brookfield (1991), however, finds it questionable and not academically grounded to expect adult learners to possess the meta-cognitive skills described by Knowles. According to him, it is not very likely that adult learners are such autonomous learners. This is confirmed by research executed by Boulton-Lewis, Wills and Mutch (1996). They investigated a group of 40 teachers of different age groups following a course at an institution in Australia and classified them according to a five-level system for personal effectiveness of learning, running from novice to expert. They found that most of the teachers functioned on the third level (being able to use several relevant independent aspects of learning in an unstructured way) not integrated into an overall structure, whereas they 
expected teachers to be at least on the fourth level (being able to use relevant aspects of learning integrated into an overall structure). The first conclusion was that learning was still seen strongly as gaining knowledge and to a much lesser extent as a way to acquire experience and understanding. The second outcome was that their adult learners did not follow Knowles' description of the adult learner as a conscious, independent and intrinsically motivated learner. It must be noted, however, that the subjects in Boulton-Lewis'(1996) research were asked to write freely about how they saw learning and how they learned. The expert level was defined as 'the integrated knowledge of learning is generalised to a new domain'. The fact that none of the 40 subjects mentioned anything that is within the definition of the fifth level does not necessarily mean that the subjects did not function at that level.

Another issue is the dependency of adult learners on educators. In order to become adequate adult learners, adults must unlearn teacher reliance (Esposito, 2005).

Learners should become more self-directed and autonomous in their learning by taking primary responsibility for their own learning. A positive self-concept is found to be important for people to become self-directed learners (Brocket and Hiemstra, 1991; Knowles, 1980), but appropriate political conditions must be met in order to make self-directed learning by people possible, as self-directed learning needs critical and free thinking, self-monitoring (responsibility) and selfmanagement (Brookfield, 1993).

Both conditions need attention in the Cambodian setting, as on the one hand it means power-shifts and strengthening meta-cognitive skills on the other.

\subsubsection{Factors influencing adult learning}

Orientations towards learning

Houle (1988) developed a three-category typology of learners, dividing them in goal-oriented, activity-oriented or learning-oriented. However, this typology does not yet explain what motivates people to learn. Boshier (1991), on the other hand, identified seven motivators that drive adults to engage in learning. Remediation of educational deficiencies and professional advancement are motivators that may explain Houle's goal-oriented category of learners, while improvement of communication skills, social contact, improving familial relationships and social stimulation may motivate activity-oriented learners, and, 
finally, sheer cognitive interest may motivate learning-oriented learners. The main reasons not to engage in learning are personal problems, lack of confidence, educational costs, lack of interest in organised education in general, and lack of interest in available courses (Valentine and Darkenwald (1990). Being aware of what does or does not motivate learners from a variety of orientations might help to support learners better in their learning process.

\section{Motivational factors influencing learning}

A positive learning orientation augments learning outcomes. All learning is influenced by emotions, either positively or negatively. Positive emotions are curiosity, need for competence, gaining confidence and involvement. Negative emotions are fear, frustration and anger (Bolhuis \& Simons, 2001). Huber and Roth (1999) found that people can be either secure-oriented or insecure-oriented. Insecure-oriented people have a high insecurity tolerance, willing to explore beliefs and solve issues. They are likely to participate in group-work, dare to ask questions, propose more and have better learning results. Secure-oriented people have a low insecurity tolerance, leading them to hold on to old beliefs and refuse to question the current (organizational) culture. In some cases, this secureoriented orientation could lead to withdrawal from processes, sabotage or resistance. Huber and Roth's descriptions coincide with the uncertaintyavoidance described by Hofstede. Most Cambodians are likely to show secureoriented behaviour. Tomassini (2001) introduced the term 'care' for facilitating a safe learning environment for staff, in the supposition that such an environment might enable people to be less secure-oriented. 'Care' reflects the safety requirement introduced by Kessels earlier.

Goal orientations also influence motivation to learn. $\mathrm{Ng}$ and Bereiter (1991) describe three goal orientations individuals use to execute learning. While task completion and instructional orientation are more extrinsic, the personal knowledge building orientation is more intrinsic. Learners possessing intrinsic orientation tend to pose questions, set goals and make adjustments where necessary. They usually have the highest learning yield (van der Sanden, 2001). Extrinsic goal orientations might be culturally stronger than intrinsic ones in more masculine environments where, for example, diplomas are seen as important. Such goal orientations should be investigated, as they influence the processes of learning. 
Other factors influencing learning

Other factors that influence learning are either personal traits, organisation of learning activities, or support in the work environment (Witziers, 2001). Individual relevant learner characteristics are intelligence, motivation, commitment towards work, self-confidence, experience and age. Beard and Wilson (2002) identified confidence in one's own abilities, good self-esteem, support from and trust in others as necessary conditions for learning. The work environment is influenced by social support, receiving feedback and the opportunity to use what was learned. Based on the findings in Chapter Three, which showed that social support, feedback and opportunities to apply acquired knowledge and skills are sometimes weak, it would be sound to provide extra attention to these 'ideal characteristics' when implementing learning trajectories in contexts like the Cambodian one.

Transfer: maximizing learning effects

Transfer remains an important issue in maximizing learning outcomes. Transfer is the process in which acquired knowledge, skills and attitudes are applied outside the context in which they were learned. Joyce and Showers (2002) identified five different kinds of transfer, running from low-level to high-level transfer. The first level merely involves imitative use, followed by more mechanical use (horizontal transfer). The third level is routine or single-strategy use. The next level is integrated use of multiple strategies (vertical transfer), followed by the fifth and final level of executive use: complete understanding of theories and selecting different strategies for different goals.

A widespread belief is that older learners are less open to change than younger ones. A 1982 study of teachers following a professional development program in the US did not find such a relationship (Joyce, Bush, \& McKibbin, 1982). The same research found that 'years of experience' (and hence probably age) does not influence transfer growth. What the research found was that enthusiastic collaborative learners have higher transfer rates. Thijssen (1996) found that application of acquired knowledge and skills is higher when the 'concentration of experience' (i.e. more varied and complex experiences over an extensive period of time) of an individual is higher. Thijssen's finding suggests that older employees with more experience have a higher transfer potential. 
Holton, Bates, Ruona and Leimbach (1998) describe all aspects of transfer as the 'transfer climate' of an organisation. Their notion encompasses more than the training components presented by Joyce and Showers (2002). Their call for creating 'opportunities-to-use' should go hand in hand with peer coaching: both take place after the actual training endeavour and are necessary for application of acquired knowledge, skills and attitudes in daily work.

This paragraph showed that personal traits and attitudes like positive motivation boost learning outcomes. What influences learning at the executive level is a matter of debate amongst many authors. Von Krogh and Roos (1996) describe three key concepts of learning, emphasizing the importance of experience, imitation and experimenting. Bolhuis and Simons (2001) apply a broader set, claiming that learning happens through culture and socialisation (as a byproduct of being part of an environment), through direct experience, through social interaction (collaboration and feedback), by reflection (thinking about acting) and through studying (learning theory). Neither system is comprehensive, but both provide important notions for learning. The next sections will focus on the most relevant aspects for this research: learning by experience, reflection and collaboration, following a social constructivist vision.

\subsubsection{A socio-constructivist approach to learning}

Previously it was shown that a corporate curriculum aims for dialogue about norms and values, and tries to find new knowledge to determine and improve the quality of the actual work (Kessels, 1996). In Kessels' vision on adult learning, individuals and teams should have the main responsibility to design their learning routes. Lowyck (2001) and Fasheh (in Merriam, Courtenay, \& Cervero, 2006) suggest that learners and educators be allowed to collaborate as codesigners of learning endeavours in communities of learners, of which learning by designing is a good example. These notions all follow a socio-constructivist idea.

Collaborative learning in a constructivist way is called socio-constructivism. The constructivist perspective aims to construct new knowledge by attributing meaning to experiences (van der Sanden, 2001). The situational perspective adds to the assumption that knowing is always intertwined with doing (Fenwick, 2003). Some constructivists claim that attribution of meaning remains personal and subjective, as it is influenced by personal learning history and social and historical 
context. Others do not deny learning by groups and talk about 'communities of practice' that refine practices, develop new ones, or discard and change practices that are harmful or dysfunctional (Fenwick, 2003). To eliminate harmful or dysfunctional knowledge, skills or attitudes, de-learning or reconstruction of previously acquired knowledge, skills and attitudes is a necessary but difficult action. People tend to be resistant to giving up previously gained knowledge and skills (Bolhuis \& Simons, 2001), although what is considered beneficial knowledge and skills can change over time (van der Sanden, 2001).

Constructivist learning involves learning in authentic contexts, which gradually increase in complexity. Many authors use the term 'competency' as the unit of result of learning. Teurlings \& van der Sanden (in van der Sanden, 2001, p. 58) describe competence as

'an integrated, coherent whole of knowledge, skills, attitudes and learning ability, based on adequate personal learning and work theories, with which an individual in certain situations and socio-cultural contexts can judge competently (interpret, analyse and classify information), anticipate on certain events and developments, and effectively can learn and act'.

Learning ability is described as the ability to develop new skills and apply those in new, unknown situations. In the definition above, socio-cultural context, the personal framework of theories and anticipating events are key components. These terms stress the importance of understanding the context and the influence of personal history, as well as emphasise the creative use of the acquired competencies in different circumstances.

The role of the facilitator of learning in a socio-constructivist approach could be that of a cognitive apprenticeship relation (Collins, Brown \& Newman, 1989; van der Sanden, 2001), in which the facilitator models behaviour, provides support and withdraws support when that is appropriate. Vygotsky's (1978) 'zone of proximal development' implies that learners should be challenged with assignments or problems that are just outside their current scope of understanding. This resembles Homan's mountain scenery metaphor presented earlier (Homan, 2005), which predicts that learning is optimal in turbulent situations that are not yet fully understood by the learners. This idea is important for the facilitator in a constructivist setting like the corporate curriculum, as the 
learners decide what they want to learn, but the facilitator should safeguard optimal learning conditions, including challenging learners within their zone of proximal development.

\subsubsection{Experience and learning}

All forms of adult learning mention experience as one of the main characteristics. Piaget (1952) claimed that experiences themselves do not lead to knowledge directly, but are transformed to knowledge through an active search for meaning by individuals. Many authors see experiences in authentic contexts important, whether or not they are combined with the 'increasing-complexity' principle (Collins, Brown \& Newman, 1989; Dewey, 1938; Kolb, 1984). This principle is an extension of Vygotsky's (1978) theory of zone of proximal development and is based on the idea that working within authentic contexts minimizes the transfer problem.

The contemporary idea that knowledge is not something 'to take from the shelves' but needs to be construed is based in Western post-modernist thinking. Post- modernists assume that there is no absolute truth. As discussed in Chapter Three, belief in absolute truths is a characteristic of societies with high uncertainty avoidance like the Cambodian one. Masculine societies feel strongly about degrees and diplomas as proofs of knowledge. In that sense Cambodians might hold more static beliefs about the value of knowledge and the need to learn from experience than their Western counterparts, although Kessels (1993) demonstrated that learning processes in the workplace are often more powerful than learning processes in formal training situations.

\subsubsection{Reflection and learning}

Learning by experience is only effective if learners attribute meaning to their experiences. Meaning-attribution from the socio-constructivist point of view is achieved in a collaborative way. It involves asking essential questions about the personal or group's frame of reference, their way of acting within the organization, their learning experiences, and learning processes. It requires personal involvement, taking responsibility for learning and acting with a critical view towards oneself and others (Belton, Thornbury Gould \& Scott, 2006; Bolhuis \& Simons, 2001). 
Reflection involves asking critical questions to one's self and others. Personal and group frames of reference and actions should be questioned (Bolhuis \& Simons, 2001). It goes beyond 'theoretical' reflections. The Kolb cycle of reflection starts with experience, upon which reflection takes place, leading to generalizations for a broader practice, which is then experimented upon, leading to new experiences (Kolb, 1984). The cycle was further adjusted by Cowan (1998) to depict socioconstructivist learning principles. The picture below shows Kolb's cycle of reflection with the added socio-constructivist notions of interactions with others.

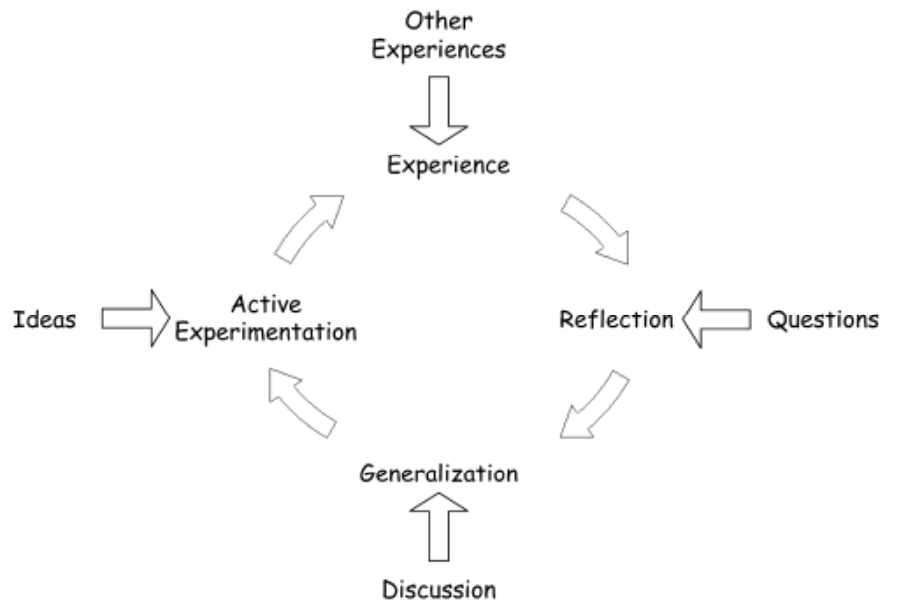

Figure 4.1 Kolb cycle of reflection with socio-constructivist notions (Cowan, 1998, p. 49)

During reflection learners focus on what exactly happened: What was the influence of culture and the subculture of the organisation? How could learning processes be influenced better? Such questions are usually asked by a 'reflective practitioner'.

Schön's reflective practitioner (Smith, 2001) is described as someone who thoughtfully considers his or her experiences in applying knowledge in practice (while being coached by professionals in the discipline). Reflective practitioners try to understand their own behaviour, in order to become more effective in a cycle of continuous growth (Ferraro, 2000). As Ferraro notes, becoming a reflective practitioner is not easy. A coach is sometimes necessary to guide a novice into reflective practice. Coaching can be done in many ways, including small and large discussion groups or study teams. These study teams can start to function as critical learning communities, in which participants share their different ways of valuing, knowing and experiencing events (Schmidt, 2000). 
Reflection on one's activities is an important aspect of learning as described by Schön (Smith 2001). Schön divides reflection into 'reflection-in-action' and 'reflection-on-action'. Cowan (1998) identified 'reflection-for-action' as a third form of reflection. Reflection could be done as a silent contemplation or aloud, which is described as important by Schön (Schmidt, 2000). The cycle of reflectionfor-action, reflection-in-action and reflection-on-action was integrated by Cowan (1998) in his model of reflection, as depicted in figure 4.2.

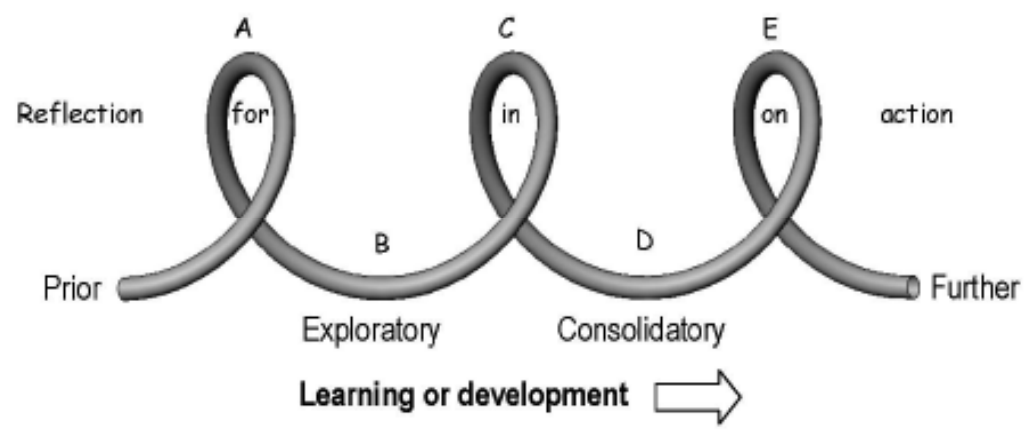

Figure 4.2 Cowan's model of reflection (Cowan, 1998 p. 53)

Cowan (1998) also introduced Kolbian cycles of reflection in between each of the big loops A, C and E, that could start at either point of the cycle. He planned those reflective activities in such a way that they contributed to reflection inand on- action.

Reflection for action is the first step: to make decisions towards a certain course of action in the (near) future and was added by Cowan (1998). Belton, Thornbury Gould and Scott (2006) refer to this stage as 'unfreezing' and value it as important for any change process. The research of Belton, Thornbury Gould and Scott is a good example of the necessity for reflection-for-action. They introduced a new learning approach for an undergraduate course, which focused on students acquiring more reflective skills. The novelty of the approach demanded the reassurance of the students by their teachers that the approach would benefit them, in order to get the approach accepted by the students and get them enrolled in learning activities. Although the results of the approach were promising, Buchanan and Scott (1998) suggested that the enthusiasm of the students might have been influenced by a preference for a variety of teaching methods, in an otherwise more conventional curriculum. This might imply that too many courses following the same approach would not all have the same effect. Nevertheless, poor attendance 
and dropout were at a minimum over successive years, which are good indicators for the engagement of learners (Belton, Thornbury Gould \& Scott, 2006).

Reflection-in-action is done by the practitioner during a certain activity and tries to ground experiences in feelings and theories currently in use. Schön's idea is that there is knowledge embodied in action, forming the link between theory and practice (Schmidt, 2000). Praxis needs to be experienced or, if that is not possible, at least simulated (Packham et al., 1989, in Belton, Thornbury Gould, \& Scott, 2006). Eraut (1994, in Smith, 2001) warns of a lack of time for reflection-in-action in certain time-pressed situations.

Reflection-on-action is done in hindsight and tries to reflect in a broader way. Although criticised by some, reflection in action and on action should both be carried out in order to learn most from a certain experience. Schön argues that understanding of reflection in action is developed by reflection-on-action (Belton, Thornbury Gould \& Scott, 2006), making them inseparable for maximal learning effect. Reflection-on-action is formalised by Joyce and Showers (2002) as the allocation of time to provide follow-up. Follow-up with peers, as they propose, provides peers with the opportunity to reflect on their actions. The importance of articulating experiences with peers is also mentioned by Smith (2001).

According to Argyris and Schön (1974) reflection is necessary to change theoriesin-use and mental maps that people hold and use to guide their actions. Theoriesin-use are implicit reflections of the beliefs people hold as mental maps. 'Espoused theories' are reflections of those mental maps we want other people to think we use (Smith, 2001). Sometimes there is a discrepancy between these 'espoused theories' and the theories-in-use. The discrepancy is the most extensive when people express their 'espoused theories' to explain or justify their actions, while their behaviour indicates otherwise (Schmidt, 2000). Such discrepancies can be identified when people are articulating their ideas after they have been observed during, for example, teaching.

The discrepancy described by Argyris and Schön (1974) is common in Cambodia, and probably in many developing countries: local staff seems to possess mental maps that do not reflect the theories of their (foreign) advisors and thus do not fully encompass the concepts, aspects and background of the theories on which the mental maps are based. Discrepancies can be caused by an incomplete 
understanding of the theories or absence of any explanation as to why things should be done in a certain way altogether.

Facilitators could facilitate reflection by creating time for staff to reflect, being open to criticism, act themselves in the manner that they wish to promote ("walk the talk') and by being tolerant towards staff making mistakes (Bolhuis \& Simons, 2001). Being open to criticism and being tolerant to staff making mistakes might prove to be difficult in the current organizational climate and culture as a whole. Van de Poll (2005) gives the example of Belarus, where people are incapable of making decisions because of their fear of making mistakes. Fear is also a common factor in Cambodian society. People are often afraid to make decisions and usually leave the decision-making to their managers or international advisors.

Learners learn from all the different kinds of activities that they do daily, but they learn most from activities that also have time allocated for reflection. Reflection provides opportunities to make (practical) knowledge and skills more explicit. Interaction strengthens the relevance of reflection outcomes (Kwakman, 1999).

\subsubsection{Collaborative learning}

Experience and reflection derive meaning through collaborative activity. Some authors describe the fact that learning from social interaction is undervalued (Bolhuis, 2000; van der Linden \& Roelofs, 2000), even though people learn mainly from activities including interaction with others (Kwakman, 1999). Learning by social interaction includes learning from working together, discussing and (critical) dialogue. But for the model of learning communities as presented by Stewart (1998) and the succession of the corporate curriculum as proposed by Kessels (1996) collaboration is a crucial part of learning, following a social constructivist approach. Eraut, Alderton, Cole and Senker (1998) conclude that learning is primarily an activity that takes place in interaction with others and is influenced by the micro-culture of the working place. In that sense learning in collaboration plays an important role in effective culture change interventions (Bolhuis \& Simons, 2001).

Mutual support and trust are very important factors for collaborative learning, as are being able to accept that one might need help and then being able to ask for 
help. If people work apart, it is necessary to hold regular meetings and keep good communication lines. Finally, honesty and transparency are essential for working and learning together (Malvicini, 2006).

People learn multiple skills from each other, such as problem solving, reflection and discussing, from working with each other in order to explore solutions to newly identified problems. These skills can be classified in three categories (Tjepkema, 2001): operational competencies, social competencies like communication skills, and improvement competencies. Collaborative learning helps to combine what is learned by experience and discussed in groups to become meaningful and crystallised.

Openness to learning, willingness to collaborate with others, sharing tasks, information and control and exploring others' ideas and meanings without confronting each other in a hostile way are important for effective collaboration (Lowyck, 2001). Trust and mutual learning are essential both for collaboration, and for reflection with each other. However, Schön (in Schmidt, 2000), argues that openness and sharing are not part of the 'ground state' of human behaviour. The ground state of most people and organizations is not collaborative. Shifting from the ground state to a collaborative and constructive state is extremely difficult. Schön shows that it is rather common to see people pretending to be rational and diplomatic, but what they are actually doing is seeking unilateral control in order to suppress criticism or negative comments. The ground state can turn into distrustfulness, defensive behaviour and avoiding sharing information or risks.

Participation as a way of empowerment has previously been discussed, but participation also applies to engagement in the instrumental processes of decision-making. The emphasis on sharing points of view is so strong that there does not seem to be much attention for conflicting interests, contradictions and power relations. Besides the fact that understanding power relations is important in the Cambodian ministerial setting, there is a real risk for false consensus and thus false ownership and commitment. Cooke (2001) identified four risks for participatory approaches. 'Risky shift' is the tendency of a group to take more risky decisions than as individuals, because there is no single person responsible and accountable. The 'Abilene paradox' makes people take actions that are contradictory to what they want, because they do not want to be bothersome to 
the group. This could lead to a situation where a group does what nobody in the group really wants. 'Groupthink' is the risk of ideas shared by a group in order to prevent conflict, without critically testing the group ideas. 'Coercive persuasion' reflects the idea of brainwashing. Old methods are put aside, a new one is introduced and must be followed by everyone. Another risk with participation is that the approach has a tendency to self-select the most articulate and politically adept participants (Hailey, 2001).

The risk of false consensus is a serious risk in conformist societies like the Cambodian one. It is likely that at least some of the issues on the Cambodian Government's agenda are based on false consensus with the international aid agenda. As described earlier, much of the development agenda is donor-driven, either after convincing the Cambodian Government that something is a priority, or by only financing interventions that are seen as important by the donor organisations. Participation as a methodology of capacity building is one of the issues that are high on the Cambodian agenda. With the ongoing development of the civil services in general and thus also the education system in Cambodia, the need to delegate work from the top to the levels below increases too. The need to empower staff is genuine, but is at least partly contradicted by an (organisational) culture that favours higher-ranking staff making decisions. Delegating decisionmaking authority calls for the willingness by the more powerful to abdicate at least some of their authority to others on one hand, and empowering staff members to make decisions on the other.

Empowering staff involves learning. Two models for learning appropriate to this research are presented in the next section: Jarvis's model of adult learning and 'mutual' cognitive apprenticeship to support such learning.

\subsubsection{Jarvis's model of adult learning}

Multiple adult learning theories combine experience, reflection and collaboration to a certain extent. The model of adult learning developed by Jarvis incorporates them most comprehensively, valuing the influences of context, experience, emotion, learning by doing and reflection. The need for collaboration is not explicitly incorporated in the model, although it is not rejected either (Merriam, Caffarella, \& Baumgartner, 2007). 
As shown by figure 4.3, the learner engages in a certain experience. Information is processed by practice and experimentation and is reflected upon. Evaluation is a continuous process, connecting all learning steps. If the learning cycle is completed, the person has learned and became more experienced, ready to meet new learning experiences.

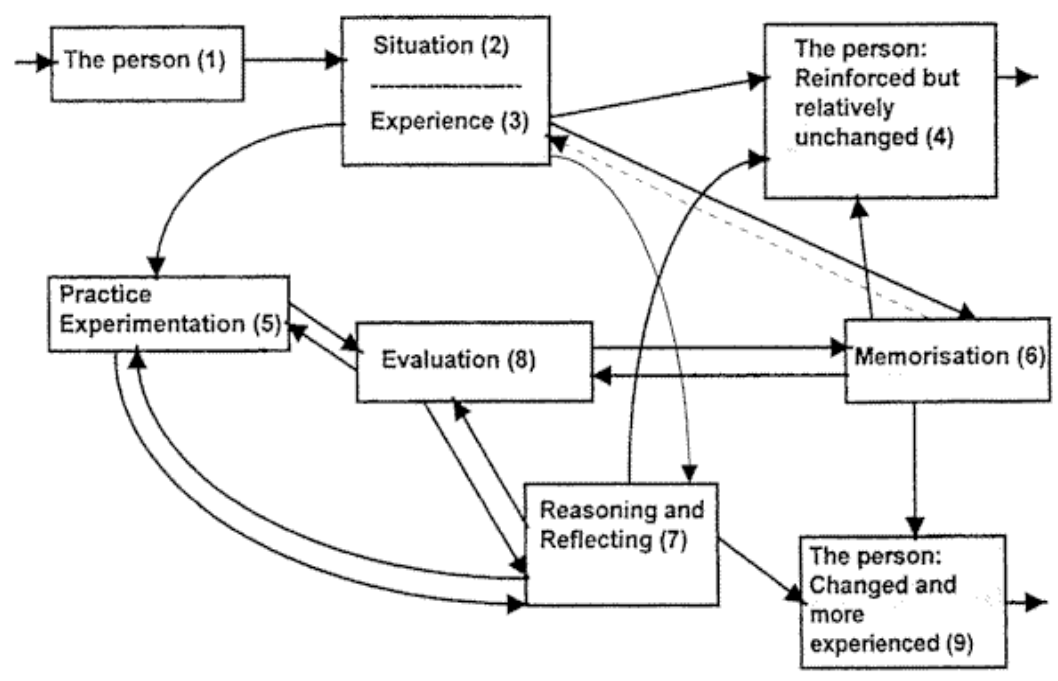

Figure 4.3 Jarvis's model for learning (Merriam, Caffarella, \& Baumgartner, 2007, p. 164)

All necessary requirements for learning as presented by Kolb (1984) are incorporated in Jarvis's model. Openness and willingness to involve oneself in new experiences are necessary to enter a potential learning situation (steps 1,2 and 3 ) and to practise and experiment (step 5). Observational and reflective skills to view new experiences in new perspectives, and analytical abilities to create integrative concepts from the observations, are necessary for ongoing evaluation, reasoning and reflection (7 and 8). Decision-making and problem-solving skills to use the new concepts in actual practice are of use when meeting new challenges with the new set of knowledge and skills (9).

\subsubsection{Cognitive apprenticeship to support learning}

Cognitive apprenticeship has been previously mentioned as a strong example of an adult learning model containing the requisite aspects for effective transfer, as it concerns situated learning in powerful learning environments with the support of experts. It is a model that helps learners to learn from experience through 
activities and social interaction. Brandt, Farmer \& Buckmaster (1993) developed a five-phase model existing of the following steps:

1. Modelling (copying of an expert)

2. Approximating (striving towards expert behaviour)

3. Coaching, scaffolding and fading (providing support and withdrawing in time)

4. Assistance on request (ask for support when needed)

5. Generalizing (using in new settings).

As the Cambodian civil service does not have extensive experience with collaborative learning projects of a socio-constructivist nature, a good model supporting such learning is imperative. As cognitive apprenticeship empowers people strongly to control and regulate their own learning, and provides high hopes for the transfer of knowledge, skills and attitudes beyond the actual learning moment, this model seems to be the appropriate choice for the research setting. One adjustment is necessary, though. In the research context described in this dissertation, all people involved are seen as both experts and learners. The local staff is expert in local habits, customs and culture. The facilitator will provide input on learning methodologies and possible learning activities. Because learning will take place in iterative cycles leading to improvement, the term 'mutual cognitive apprenticeship' might be more appropriate, sharing knowledge and skills when appropriate between all participants.

\subsubsection{Design principles from the adult learning perspective}

Analysis of (adult) learning theory leads to the following design principles for the scenario:

- Apply a socio-constructivist approach using the teams' and learners' zones of proximal development, in order to stimulate learning. Such an approach utilizes the teams' and learners' experience to come to collaborative learning.

- Create a positive, motivating and safe learning environment for learners to support such socio-constructivist learning, as in Cambodia fear and lack of motivation are common issues that influence learning negatively.

- Strengthen meta-cognitive skills and unlearn teacher-reliance, in order to allow learners to take control over their own learning.

- Create a balanced team of learners, reflecting differences in age and experience. Contextual experience is an important source of information possessed by MoEYS staff, but has been neglected in the past. This neglect is 
one of the reasons why previous professional development activities were often not effective.

- Allocate moments for and support reflection for, in, and on action, in order to optimize learning. Reflection is a skill that is not possessed widely by MoEYS staff.

- Use Jarvis's model of adult learning as a checklist while developing the scenario, as it combines the other design principles presented here.

- Apply the cognitive apprenticeship approach to provide support to learners.

\subsection{CULTURALLY RELEVANT LEARNING}

\subsubsection{The necessity for culturally relevant learning}

Most of the literature on adult learning is based in Western learning communities and on white middle-class experience (Flannery, 1994). Many sources have a focus on emancipating marginalised groups dominated by the host culture (Amstutz, 1999; Guy, 1999a) and hardly any on building capacity or professional development at a policy level. Theories and approaches striving for a more equitable society by liberating and empowering marginalized groups (see Amstutz, 1999) might be in themselves Western concepts as to how marginalized people should evolve.

Many contemporary authors reject the idea of a generic adult learner having universal characteristics and traits (Guy, 1999b). Most assumptions about adult education remain Western-based and concerned with the concepts of individuality, autonomy and rationality (Merriam \& Ntseane, 2008), whilst aiming for the inclusion, participation and empowerment of the learner (Guy, 1999a). These assumptions might not fit anything other than Western settings. A wonderful example, which is also applicable to the Cambodian setting, is that of Chinese-American learners, who feel uneasy about leading a class discussion because of different cultural values. Such discussions might be seen as questioning the teacher's authority and as a waste of time because the teacher could be teaching in the time taken for discussion (Pratt, 1992). 
Most adult education activities ignore culture as a significant factor (Amstutz, 1994; Pratt, Kelly \& Wong, 1998), although the socio-cultural context of learners has a strong influence on their motivations, needs, goals and perspectives towards and on learning. Understanding these and their implications for adult learning is necessary for adult learning to become culturally relevant (Amstutz, 1999). If we take the influence of culture for granted, i.e. do not recognize its importance in these capacity building processes, we can safely assume mismatches between what is trying to be done and the result of professional development activities. Thus, Amstutz (1999) calls for culturally relevant adult learning instead of imposing systems or approaches on cultural contexts that are too diverse from the context in which an approach was developed. She gives some suggestions, such as helping students to question theory relative to their own cultural experiences, teaching in non-dichotomous ways of knowing, acknowledging different forms of knowledge, using a variety of instructional strategies, constructing supportive and safe learning environments and continually reviewing educational goals.

Hofstede (1986) identified four levels of problematic situations that can arise with cross-cultural learning. The first level involves differences in the social positions of both teachers and students in both societies. The next level warns about different expectations in the relevance of training content (curriculum). The third level deals with differences in what is important and expected as cognitive abilities of teachers and students. The final level involves differences in expected patterns of interaction between teachers/students and students/students.

In cases where the culture of the adult educator and her learners differ, Marchisani and Adams (1992) propose a model to assist educators to conceptualize a learning environment from a culturally relevant perspective. The model, developed to address cultural problems in multicultural learning settings in the US, consists of four elements to take into account while working with different cultures of learners and teachers: the educators' cultural identity, the learners' cultural identity, the curriculum and instructional methods and processes.

In the work of both Hofstede (1986) and Marchisani and Adams (1992), curriculum, differences in cultural beliefs, expectations and identities are mentioned to be of influence on learning. Differences in social positions are an important extra facet that Hofstede brings forward, although he limits this to the 
teacher-student dimension where a power difference exists between the two groups. The inequality however, exists also when an expatriate and a local colleague work together on the same project. This is mainly based on beliefs from both sides that the expatriate has higher knowledge and skills than the local colleague, and partly on the salary difference that usually exists between the local colleague and the expatriate. Such differences should be overcome in order to learn from each other and develop interventions that are relevant for the context.

\subsubsection{Design principles from the cultural relevant learning perspective}

Analysis of cultural relevance theory leads to the following design principles for the scenario:

- The researcher needs be aware of culturally different interpretations of concepts like individuality, autonomy and rationality.

- The researcher needs to understand the socio-cultural context of learners and its influence on motivation, needs, goals and perspectives towards learning.

- The researcher needs to adjust theories and models used in the scenario to fit the specific cultural context in collaboration with learners, by questioning theory, teaching in non-dichotomous ways, acknowledging different forms of knowing, using a variety of instructional strategies, constructing supportive and safe learning environments and ongoing adjustment of educational goals, when necessary. This chapter abundantly showed the limited applicability of Western-based theory to other cultures. 


\section{CHAPTER 5 \\ Portraying the enacted scenario}

\section{$5.1 \quad$ INTRODUCTION}

The previous chapters investigated the context and literature relevant to the research described in this dissertation. The analyses of the context and literature informed a set of design principles. The design principles helped to construct an initial scenario design that was then further developed into the enacted scenario by the community of learners during eleven working group meetings, three preparatory meetings to develop the four-day Training Guideline training, and the training itself. This chapter describes the design and development process of this enacted scenario.

Figure 5.1 shows how the design and implementation phase was based on the identified design principles during the preparatory phase; how the design and implementation converged into the 'enacted scenario'; and how it was followed by the evaluation phase.

The design and implementation phase is depicted as a circle because development and implementation of the scenario was a simultaneous and iterative process. The initial scenario design was informed by the design principles, but occasionally the design principles were adjusted by the experiences during the enactment of the scenario. The reciprocal arrow between the design and implementation phase and the design principles reflects this relationship. 
The scenario was further shaped and 'fine-tuned' by the 'real-life' events as they took place during implementation, as will be described in the rest of this chapter. Formative evaluation during the implementation of the scenario provided important information that was used to adjust the scenario. This 'fine-tuning' is the strength of educational design research, and makes it possible to come to a relevant, consistent, practical, and effective scenario.

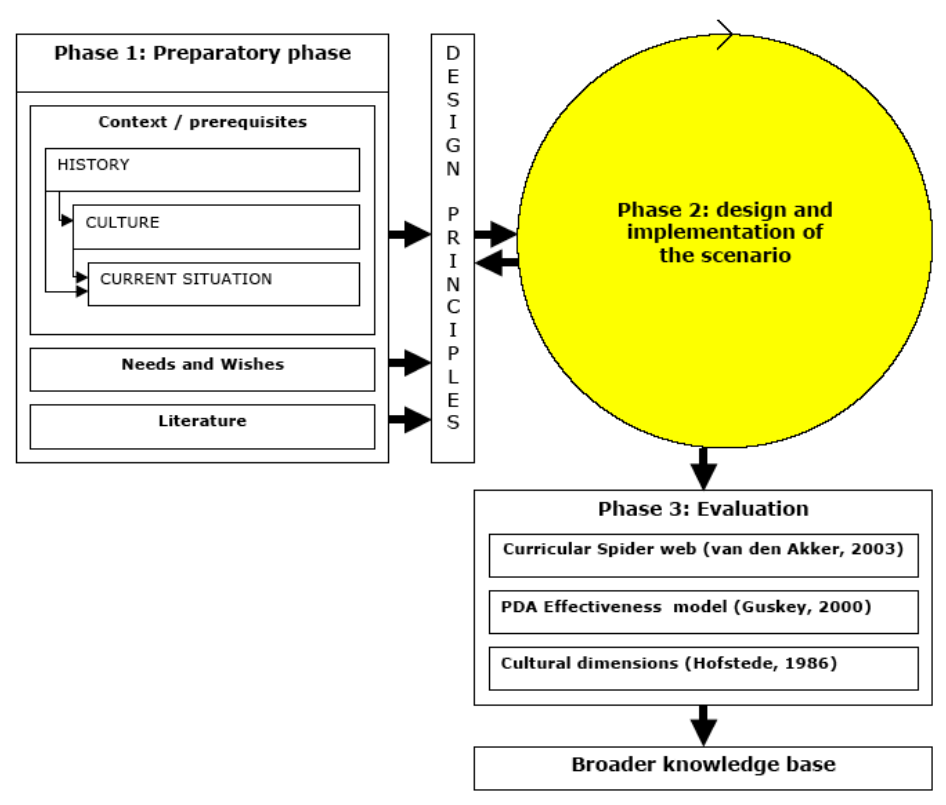

Figure 5.1 The place of the scenario design and implementation in the research

In Chapter Two, it was explained that the different phases of the research presented in this dissertation sometimes overlapped. These overlaps were sometimes caused by external influences, but do also belong to exploratory educational design research. An example of external influence was the slow start of the intervention, as it needed to be embedded in the Ministry of Education, Youth and Sports (MoEYS) capacity building plan, followed by the sudden push by the Directorate General of Education (DGE) to start the formation of the working group. Such decisions had tremendous impact on the research process. Neat separation of research phases was impossible, as important documents emerged long after the initial literature analysis took place, envisaged approaches turned out to be much less appropriate and needed to be adapted or even changed, and the background of people involved was sometimes found to be different from what was expected. 
One of the design principles was to establish a community of learners that would bring representatives of the various Departments together. After that, twelve working group meetings were planned over a period of six months to allow the building of trust and provide time for a collaborative learning process. The collaborative learning process was informed by notions from the corporate curriculum approach and socio-constructivist learning principles. In order to reinforce application of acquired knowledge and skills, the four training components of Joyce and Showers (2002) were incorporated. Study of theory, modelling, practice and peer coaching took place during the working group meetings, but extended practice also occurred during three preparatory meetings for the preparation of the four-day Training Guideline training for MoEYS staff and the training itself. The creation of a safe learning environment in which learners could share ideas was important. The cognitive apprenticeship model was adopted to support learning. Meta-cognitive, regulative and communicative skills also needed to be strengthened.

The scenario aimed at strengthening knowledge about professional development and extending the methodological repertoire of the group members in order to become better facilitators of learning. Table 5.1 shows the components of which the scenario was composed. The twelve planned meetings (in reality eleven, as one meeting was cancelled) of the working group were conducted between February and July 2007, the preparatory meetings in February and March 2008, and the training was conducted in April 2008. The content is summarized briefly per meeting, and the tools for data gathering are given per component.

The next sections will describe how the initial scenario evolved into the enacted scenario. First the start of the scenario is presented, followed by a description of the meetings, preparatory meetings and the Training Guideline training. How learning actually took place in comparison with the design principles is described in the last section of this chapter. 
Table 5.1 The components of the scenario, including content and data gathering tools

\begin{tabular}{|c|c|c|}
\hline Component & Content & Data gathered by \\
\hline $\begin{array}{l}\text { Working group } \\
\text { meetings } \\
12 \text { meetings } \\
\text { between } \\
\text { February and } \\
\text { July } 2007\end{array}$ & $\begin{array}{l}\text { Meeting 1: Objectives of the working group; } \\
\text { time schedule; levels of evaluation; } \\
\text { Meeting 2: Evaluation, when to evaluate; forms } \\
\text { of evaluation; evaluation models; } \\
\text { Meeting 3: Steps in training; making first } \\
\text { prototype of evaluation form; } \\
\text { Meeting 4: Revision of evaluation form } \\
\text { prototype; identifying characteristics of active } \\
\text { participants } \\
\text { Meeting 5: Preparing sessions and activities; } \\
\text { steps taken during organizing PDAs } \\
\text { Meeting 6: Exploration of learner-based } \\
\text { activities like icebreakers, energizers and wrap- } \\
\text { ups; steps in organizing PDAs; } \\
\text { Meeting 7: Identify definitions of monitoring } \\
\text { evaluation, follow-up, and reporting; } \\
\text { Meeting 8: Definitions, background and steps in } \\
\text { reporting; } \\
\text { Meeting 9: Special participatory workshop at } \\
\text { HealthNet/TPO: participatory techniques; } \\
\text { Meeting 10: Background of follow up and } \\
\text { discussion activities; } \\
\text { Meeting 11: Identify the structure of the } \\
\text { Training Guideline document and discuss the } \\
\text { draft; } \\
\text { Meeting 12: Cancelled. }\end{array}$ & $\begin{array}{l}\begin{array}{l}\text { Observations of } \\
\text { each meeting }\end{array} \\
\text { Midterm } \\
\text { evaluations } \\
\text { Evaluation forms } \\
\text { End evaluations } \\
\text { Informal } \\
\text { conversations }\end{array}$ \\
\hline $\begin{array}{l}\text { Preparatory } \\
\text { meetings } \\
3 \text { meetings } \\
\text { between } \\
\text { January and } \\
\text { March } 2008\end{array}$ & $\begin{array}{l}\text { - Preparation of the four-day Training } \\
\text { - Allocation of sessions to group members } \\
\text { - Checking Khmer version of the Training } \\
\text { Guideline document }\end{array}$ & $\begin{array}{l}\text { Observations } \\
\text { Informal } \\
\text { conversations }\end{array}$ \\
\hline $\begin{array}{l}\text { Training } \\
\text { Guideline } \\
\text { training } \\
1 \text { training of } 4 \\
\text { days in April } \\
2008\end{array}$ & $\begin{array}{l}\text { - Dissemination of the Training Guideline to } \\
\text { MoEYS staff } \\
\text { - The training is facilitated by the group } \\
\text { members in order to evaluate their utilization } \\
\text { of acquired knowledge and skills }\end{array}$ & $\begin{array}{l}\text { Observations } \\
\text { Informal } \\
\text { conversations }\end{array}$ \\
\hline
\end{tabular}




\subsection{THE START OF THE SCENARIO}

\subsubsection{Situating the scenario in the Ministry}

During the first year of the research, the researcher invested a large part of his time in gaining understanding of the Cambodian and, specifically, the Ministry of Education (MoEYS) context. This process continued throughout the research, but the first year was crucial to construct the initial framework of understanding. It was found that power issues in the form of hierarchy are very important in MoEYS. Coupled to this hierarchical system is a fear of making mistakes, either in hierarchical issues, or in work and personal contact in general.

Furthermore, it was discovered that it was necessary to get official approval from senior officials, before any intervention could be started. Without such approval, important support for the process would be missing, making implementation impossible. Therefore, it was important to embed this intervention in the MoEYS capacity building plan. It was thought that since the intervention was embraced by the Deputy Director and the Capacity Building Coordination Group (CBCG) of the Directorate General of Education (DGE), and formalised in the capacity building plan 2007-2010 (Berkvens, 2007a), the project had sufficient approval and would receive all required support. Yet, the working group never received 'formal status'. This had consequences not only for the attendance of group members, as members were often called away to other meetings that did have this status, but also for the location where meetings were held as well as available budget. The responsible senior officials participated in CBCG meetings only, but not in either in the working group meetings or in the four-day training one year later. This was to do with over-stretched agendas, but also with a hierarchical divide between senior officials and their staff. Not having a formal status also made it impossible to provide certificates to the group members after the intervention. Although there is not yet an HRM-system in MoEYS that recognizes these certificates or offers incentives for acquiring them, such certificates are perceived as important by MoEYS staff. All group members repeatedly asked for these documents to prove their enrolment in the working group. It is highly likely that the desire to have certificates is status-related. Three members wanted to have a framed document with a passport photograph of them on it, so that they could put it above their desk for others to see. 
Keeping the CBCG and the Deputy Director of the DGE informed during the initial design phase was extremely important for the intervention to maintain their support on the one hand, but also provided opportunities for the researcher to stress the importance of a more integrated approach of HRM on the other. Although such an approach had been discussed with senior officials on more than one occasion by the researcher, it was also clear that decisions about such fundamental changes in the organisational system were beyond the power of the people the researcher worked with, and certainly beyond the scope of the scenario described in this research. The pre-conditions for this learning scenario were thus not different from those of other professional development endeavours. There would be no change in career paths or payment of incentives or extra bonuses.

Including the Deputy Director of the DGE and other stakeholders like UNICEF in the process was important to ensure external consistency of the scenario. Because all stakeholders were kept informed on a regular basis and had the possibility to provide input, the scenario was supported and the outcomes shared and appreciated.

\subsubsection{Characteristics of the community of learners}

The composition of the working group as a community of learners was based on a selection procedure that was out of the researcher's control. Instead of being selected by the researcher, the working group members were chosen by their Departments. This reinforced the risk of selection bias: The members were selected on their suitability and, as such, were not representative of MoEYS staff. The tendency to work with the better-educated local staff in developing countries was described in Chapter Four. On the positive side, it meant that most working group members had relatively strong English language skills, and had at least some experience in developing and providing training.

Fortunately, the working group of nine people (subsequently seven due to dropout) was a good mix of young and older, male and female, and experienced and less experienced MoEYS staff. Four of the members were female, six were older (around 50), and three group members were between 24 and 35. Two members held a master's degree, five had a bachelor's degree, and two had a high school degree. One of the members had studied abroad (in Russia). All had 
been working with expatriates on the development or conduct of training and workshops. Three members had never developed training modules before, but all members had delivered training or workshops in MoEYS. Three members had been working at MoEYS for more than 25 years, four around 15 years, and two for less than 8 years.

Three group members dropped out, one of whom was replaced. The second member was not replaced as her Department was still represented by another group member. The third member was not replaced, and led to one Department not being represented after the first three meetings. The overall team of learners, however, formed an effective community of learners, as they were able to work on the scenario and meet the goals set by the Deputy Director and the CBCG of the DGE.

The nine working group members were interviewed at the start of the scenario in February 2007. The interviews were conducted to acquire profile sketches of the group members and to gain a better understanding of the starting position, expectations and experience of the group members. This included any experience they may have had as trainees and trainers. The findings are presented below.

Expectations of being part of the working group

The main expectations of being part of the working group were said to be: 1) to gain more experience and capacity; 2) to learn how to organise effective training; 3) to learn how to make a plan for a professional development activity (PDA); and 4) to learn new skills and activities. Some remarks showed the limitations of previous learning endeavours the group members had undergone: 'I always write down what we hear or know, but I want to learn how to do the things myself', and 'I want to understand better, because the training in Japan was sometimes too fast and too difficult for me.' One remark showed the dependency on others to decide what would be important to learn: 'I would like to learn more, but do not know what because you will do (because you know about this)', referring to the researcher as the person knowing what should be learned.

\section{Experience with successful PDAs}

The group members were asked to identify characteristics of successful professional development activities they had implemented in the past. Most of the group members claimed that these PDAs had been effective, because 
'participants applied the knowledge they learned in their work'. However, the prerequisite for such outcomes was mentioned by the group members to be the participants' commitment to apply the results of the training in their work'. Most of the endeavours described included a pre-test and a post-test, to measure the effect of the PDA. Successful training consisted of a mixture of activities, including practice, field visit and group work. Some interviewees mentioned role-plays, brainstorming, icebreakers and games, but all said that group discussions were used as the main methodology for sharing information.

The majority identified a good selection of activities to be important. One interviewee said that 'the activities made people understand', another said that 'we saw and experienced the difference in the work of the people: Before they were passive do-ers (learners), after (and during) the training they became active'.

Support to trainees, either by facilitators or peers, was also identified as being crucial: 'If they cannot do something, we help them to find a solution', and 'If another school resolved ... the problem, they can learn from each other'.

One of the interviewees said that 'education is very important to create change'. According to the group members, the most notable changes were seen after 'the participants practised and did' activities during the training, leading to 'a change in knowledge, skills and behaviour'.

Experience with unsuccessful PDAs

One of the group members described training as 'not successful if trainees do not use the knowledge and skills they learn in training'. The two main constraints to develop and provide effective training were said to be lack of time and limited funding. One of the interviewees described the time constraints as follows: 'Time needed and demands of other duties should be weighed against each other. Leaders of workshops have other jobs...' But the budgetary constraints had more widespread consequences: 'There is no time to practise, not enough materials to produce learning aids' and 'no certificates'. The lack of teaching materials contributed to time constraints: 'If no handouts or information on paper, trainees are writing all the time, so no time to do other activities'. Another interviewee thought that sometimes results are expected too soon. For example, at a gender training to leaders (of Departments and schools), 'we wanted to change attitudes of people, but this turned out to be very difficult. This kind of change does go very slow, and is especially difficult for some male leaders'. 
Participants' learning needs were not assessed for any of the unsuccessful PDAs. This led to situations in which 'the training topic was not relevant to the participants', for example at a training session about management for primary school teachers.

Because the learning needs of the participants were not known, the learning activities were not adjusted accordingly. The learning activities were limited to theory and group discussions. All PDAs were characterised by an over-emphasis on lectures. They were perceived as 'boring' and 'too long-winded': 'The participants received the theory only, they did not practise, so there was no transfer and no creativity'. Their boring character was reinforced by the lack of energizers: '(It is) theory only, only small activities like a song and a poem are used'. In at least one of the described unsuccessful PDAs described, the lack of well thought-through activities led to incomprehension in the participants. As one commented:

'During one workshop there was no group discussion; it was teacher- or trainercentred. Then the trainer asked: "Any questions?" And there were none. So he moved on to the next part. But there were no questions because nobody understood.'

This example also shows the important influence of reluctance to admit ignorance or cause the facilitator to lose face felt by many participants. They had difficulty in saying they did not understand the facilitator. One of the members said that the technique used to check if people understood everything is therefore important: 'If people have any problems, they could ask or write an anonymous letter when they are afraid.'

At the same time, the over-reliance on group discussions was said to be ineffective. About group discussions members said: 'Many presentations (of group work) were like a lecture, too long talking to participants', and in some cases 'discussion groups took so long, that time for presenting lacked'. One group member said that 'discussion groups are not always the best. Sometimes a workshop takes five days, where three would be enough'.

Sometimes the participants of PDAs were seen as the reason why training was not effective: '(The participants) are lazy sometimes', 'do not understand' , 'are 
not concentrated', or 'do not work together easily'. Furthermore, some of the participants were said to be 'uninterested because they were invited by provincial leaders without having knowledge of the subject', or 'attended only to get money'. Sometimes participants 'came for the second time (to receive a 'per diem' payment) and were not motivated'. One of the group members said that the training topic should be relevant: 'If the training topic is not relevant to the participant', then training will not be successful. According to some group members what should be trained in detail should be assessed using training needs assessments, but others adopted a top-down approach: 'We (at central level) know what they do not have (what knowledge they possess and what they miss), so what we need to teach them'.

The group members were also critical towards themselves as facilitators. The lack of theoretical background and not using feedback mechanisms were mentioned often: 'Trainers did not know about the topic', and: 'Trainers did not discuss the work of the participants'. They also felt that their PDAs were badly organised, learning activities not thoughtfully selected and participants not well enough supported. One of the participants said: 'I made bad materials and had bad planning management'. Another blamed himself for providing 'a training (session) that was not participatory', and that he 'did not share his lessons learned and did not give any new ideas to participants'. Most of the interviewees acknowledged the need for preparing training sessions well: 'If we (trainers) follow the prepared schedule well, and the people responsible for a topic do good, the training is successful'.

\section{Experienced capacity building on professional development}

As previously mentioned, most group members had worked with expatriates on professional development before. In these cases, the support of a foreign expert was seen as contributing to the weakness of a PDA. As one participant commented:

'When a TA (technical assistant) speaks English, sometimes (he uses) too many technical terms to translate, sometimes words are unknown (to us).'

On the other hand, one of the interviewees said that the PDA remained unsuccessful because 'there was no consultant'. 
Capacity building on professional development was not only provided by expatriates, but also hosted overseas in, or imported from, other countries in the region. The experience of one of the interviewees was negative: 'Some training delivered from Thailand can be done in Thailand, but not here in Cambodia, because we don't know and do not have the money and skills to do'. Often, these PDAs are too high-level: 'I did not understand all of the training I received overseas' or 'the English was too difficult for me'.

Ideas about monitoring, evaluation and follow-up

Participants' knowledge about monitoring, evaluation and follow-up and the experience with these processes were also assessed in the interviews. Evaluation was found to be the best understood by the interviewees, although evaluation was often confused with monitoring and follow-up. The majority of the group members thought that these three processes (monitoring, evaluation and follow-up) should take place in a linear order. One interviewee had a more accurate perception of how the three processes are interrelated, and said about follow-up: 'We give recommendations during monitoring and discuss the results at the Department'.

Evaluation was described in various ways such as: 'comparing the results of pretests and post-tests', '(we evaluated) every training day with a form, and at the end of the course', and 'we sit together with trainers and discuss difficulties and questions a trainer could not answer'. Long-term effects of training were not assessed by any of the Departments: 'We never evaluate long-term (for long-term learning outcomes), but only after the workshop (directly at the end)'. This automatically implies that the actual application of acquired knowledge and skills at the workplace after training is not regularly assessed.

Monitoring and follow-up were much less well understood. When asked about examples, eight out of ten interviewees gave examples of evaluation. Only two interviewees gave appropriate examples of monitoring: '(To) go to school after one month and observe classroom management, lesson plans, motivation and room organisation', and 'During (training) we identify people with weak understanding'.

Follow-up was mainly seen as 'support on request', or 'bulk' supply in the form of refresher training endeavours or extra workshops. More individual forms of 
follow-up did not take place. One group member implied that evaluation and follow-up could not take place because monitoring was also not executed: 'After some workshops we do not know if participants implement what they learned, because we do not go and see'. Only one group member connected follow-up to monitoring activities.

Although monitoring activities were seen as important, lack of money was mentioned nine times as the main reason for not carrying them out. But there is also a cultural issue at work: One group member said that 'speaking out is not always accepted, we do not select or decide what to put, we put everything (in documents). This is the reason we do not reach goals, because we all think we know better... also in the working group'. This example shows that, beside a financial reason, there might be a hierarchical influence involved in not executing monitoring, evaluation and follow-up. In the beginning of the scenario, many discussions on the contents of the Training Guideline were indeed on including all comments, in order to satisfy all group members. But during the process, group members became more direct towards one another and decisions were more collaboratively made. Time was found to be important in overcoming hierarchical obstacles. When working on the checklist for the preparatory stage of organising PDAs, for example, one of the more senior group members wanted to put the steps in a certain sequence with which the other group members did not agree. During the next meeting, the more senior group member agreed with changing the sequence in favour of the others' comments given in the previous meeting.

\subsubsection{Characteristics of training endeavours and workshops}

The researcher observed six training courses and workshops that were conducted by group members between April 2006 and March 2007, in order to gain insight into the level of their facilitation skills. Most of the observations gave a strong impression of an overemphasis on lecture and group discussion. There were some exceptions that have already been described in Chapter Three, but some other observations are worth mentioning.

The use of training games was found to be very limited during MoEYS PDAs. In four of the six observed activities, no icebreakers were performed at the beginning of the workshop or training sessions. One of the workshops that did include an icebreaker started with two stories told by different participants, while 
the rest of the participants remained seated and listened. The other training course, for 'core mother volunteers' of an early childhood education program, started with a short game. It was only in this 'core mother' course and not in any of the other endeavours that energizers and games were included.

Time planning and time management were both problematic for the facilitators and led to overstretched agendas. None of the facilitators was able to stick to the prepared schedule. The most severe case was a three-day workshop to develop a science curriculum. Much of the work needed to be done by the facilitators after the workshop, because too much working time was lost on group discussions and presentations.

No facilitator meetings were held either for preparation or for evaluation during and after the PDAs. With the exception of the science curriculum workshop, the facilitators of the training courses and workshops left together with the participants and only returned for a short time in the afternoon or the next morning to prepare flipcharts. This lack of coordination and preparation led to overlaps in sessions, and confusion between facilitators.

When participatory activities were used, they tended to be repetitions of the same methodology. After a while, especially during multiple-day training endeavours, this became boring and the learner-based character lost its power. The 'core mother' workshop is a good example. This was the most participatory and learner-based of the observed six PDAs. But small group work repeatedly involved matching pictures to sentences that were printed on paper, and then presenting those matches to the big group. Some participants were observed to whisper comments like 'again?!' and 'boring' to one another, after the methodology was used twice.

Participants from the cities were usually shy and avoided asking questions and even more so when from the provinces. Facilitators did not always acknowledge or realize this, which led to uncomfortable situations for participants. Demanding answers from participants by addressing individuals directly was frequently observed, and in all cases led to nervous giggling and evasive answering by the participants. 
The facilitators asked the researcher to give his opinion after many of the observed PDAs. There were usually a lot of positive things to say, which were highly appreciated by the facilitators. But the facilitators were a lot less open to the feedback on things that could be improved. For example, when the facilitator of one of the observed workshops asked after the workshop had ended: 'what do you think?' and the researcher started with the positive feedback, the facilitator was interested and asked for examples of those positive remarks. The critical feedback, however, was met with a short 'oh' and short nods. When the researcher started to give examples, the facilitator thanked the researcher and asked when the meeting of the next working group would be.

Some of the PDAs observed were facilitated by expatriates, or included foreign participants. In almost all PDAs, the foreign experts dominated group work (including group work presentations) and discussions, for example during a workshop at the National Education Congress in 2006. Ten minutes after the group work had started, the two foreign consultants present left the room to smoke and talk among themselves in the corridor, on the other side of the glass partition, in plain view of the participants. When it was time to present the findings of the group discussions, the consultants returned to the room where the Cambodians had been working in groups, and started interrupting the presentations to give their opinions as if the work that the Cambodian participants had done was not of any relevance. Another example of the way in which Cambodian participants were sometimes excluded from the discussions was observed during a meeting about the provision of food supplements through schools. The foreign participants were engaged in strong debates, with the Cambodian participants keeping silent. Some of the Cambodian staff even started to do other work in the back of the room.

\subsubsection{Conclusion at the start of the scenario}

The first interviews and the observations of PDAs provided insight into the experience of the group members, their expectations and their needs. It can be concluded that all group members experienced 'good practice' examples, which were characterised by possibilities to apply acquired knowledge and skills in work and a strong selection of learning activities. 'Bad practice' examples, on the other hand, typically embodied material shortage, overemphasis on lecturing and group discussion and lack of assessment of learning needs. Demotivated learners 
or facilitators also reduced potential learning. The group members had internalized misconceptions of monitoring, evaluation and follow-up, and lacked a sound conceptual framework about professional development.

The outcomes of the first interviews and training and workshop observations confirmed the appropriateness of some of the design principles used to develop the initial scenario design and further shape the enacted scenario. The need to work collaboratively together, share knowledge and skills, the need for an extended repertoire of training methodologies and facilitation skills, and the provision of opportunities to practise newly acquired knowledge and skills, were identified as important. The need for sufficient time for learning was also confirmed. An important finding was the need for a conceptual framework about professional development, as many definitions, processes and approaches (for example on monitoring, evaluation and follow-up) were not coherently understood.

The next sections of this chapter form a reconstruction of how the 'enacted' scenario was established. Such a reconstruction allows us (or the researcher) to draw conclusions about the design principles that were at the base of the scenario.

\subsection{THE COMPONENTS OF THE SCENARIO}

\subsubsection{The working group meetings}

The meeting schedule

The meetings of the working group were spread over a half-year period from February to July 2007. Such a time span was chosen, in order to allow enough time to build trust, and complete the micro-cycles leading to balanced outcomes and improved learning. It was found that the more prolonged time scale was essential for establishing trust, through building personal relationships between the researcher and the group members, but also between the individual group members. Time was indeed crucial for learning. During micro-cycles, products or methodologies were developed or adjusted, in a cycle of selection, adjusting, testing, revising and testing again. This will be explained in detail later. 
One of the originally planned twelve meetings was cancelled, due to schedule conflicts of the group members. One of the meetings (meeting nine) was turned into a special 'participatory techniques' meeting. Usually, the meetings lasted around two to three hours and were planned for either a morning or an afternoon, on varying days of the week, in order not to interfere with fixed weekly activities. In reality, the date and time for the following meeting were always decided at the end of the previous meeting. The prepared schedule was only a guiding principle. Invitations for each meeting were sent to each member by the researcher a few days in advance, indicating the subject, date, time and place of the meeting. Even then only half to three quarters of the group members would be present at each meeting. That tended to be the same group members every time. All meetings started twenty to thirty minutes later than scheduled, and sometimes group members were telephoned to remind them that the meeting was about to start. These late starts might have been the result of the aforementioned lack of formal status of the working group. In general, meetings in Cambodia start only ten to fifteen minutes late, and it is not customary to call people.

\section{The meetings}

All meetings were held in the small meeting room of the DGE and began with an icebreaker or energizer, followed by a recapitulation of the outcomes of the previous meeting and the presentation of the goals and objectives of the current meeting.

The first meeting started with an icebreaker in order to introduce the group members to each other. An initial exploration of how PDAs typically were developed in the individual Departments was made by groups of three using 'post-it' notes on paper, and the results were presented to the big group. Then, a short presentation on evaluation was given by the researcher, after which two groups were formed to develop the first prototypes of the evaluation forms, one for the short-term (provided directly at the end of a PDA) and one for the longterm learning effects (provided three to six months after a PDA). The forms were more rudimentary 'lists' of questions that the two groups then presented to the others (annexes 8 \& 9).

The second meeting started with a short lecture in which some more background on evaluation forms and possible response formats was provided, in order to enable the group members to develop sound questions and response formats. 
This was followed by a more thorough lecture on evaluation, including evaluation of the PDA planning phase, formative phase and end phase (summative evaluation). The provision of such a framework was necessary in order to strengthen the members' conceptual framework on these processes (annexe 12). The five critical levels of professional development evaluation model (Guskey, 2000) was then used by the two groups to continue working on the evaluation forms:

The group working on the long-term evaluation form was struggling with the questions about organisational support. With the model of Guskey, they were developing the right questions (field notes $2^{\text {nd }}$ meeting $p .2$ ).

This work provided the second prototypes of the forms that now looked more like forms, with dichotomous 'yes/no' answers, Likert scales and open questions. Still, questions and answers needed to be balanced, as a question like 'Is the training course useful for your Department', had a Likert answer scale of four categories, from 'excellent' to 'poor'. The forms were then presented and taken to the Departments for feedback.

During the third meeting the groups finished the evaluation forms and presented them to the group (annexes $10 \& 11$ ). The next activity started with presenting the outcomes of the initial exploration of how PDAs were developed from the first meeting. Now, the group members were asked to write every step on a separate piece of paper, and put the steps in order. The members were asked to literally walk through the steps and replace, remove or add steps. While walking through the steps of organisation, people found a number of differences in organisation between the different Departments. Some Departments first organised a PDA, and then proposed the activity to donors, whereas others first secured funding and then started organising the PDA:

There was thorough discussion on where the budget should go. Finally it was split between budget planning as step two and budget description as step fourteen (field notes $3^{\text {rd }}$ meeting $p$. 4).

Similarly, much discussion took place on when the necessary materials should be decided upon: 
Two of the group members insisted on doing that as step thirteen, although the majority felt it should be step eleven. During the discussion, the two group members repeatedly put it back to position thirteen (field notes $3^{\text {rd }}$ meeting, $p$. 4).

The 'stepping' approach was deliberately chosen in order to identify differences between Departments, but also to actively involve all group members in the thinking process to come to a checklist for the PDA preparation phase. This activity was followed by a group discussion in order to identify what differences existed between the Departments, why these differences existed and how possible issues behind these differences could be resolved in order to arrive at a general strategy for organizing PDAs. The outcomes were put into a first prototypic checklist for the planning phase (Annexe 14).

The draft checklist that was developed during the third meeting was sent to the participants together with the invitation for the fourth meeting in order to allow the group members time to discuss the outcomes in their Departments and collect feedback. The fourth meeting started with the members giving feedback on the forms. Therefore, each page of the forms was put on a flipchart, and the group members were invited to write their comments on the charts. The outcomes were discussed and questions were adjusted:

Two of the group members were discussing what kind of answering format should be used for questions twelve, thirteen and fourteen of the long-term evaluation form. They are not able to come to an agreement on how to ask the question, until one of the other group members proposed to use a dichotomous yes/no answering model for these questions. That was agreed upon by the group (field notes $4^{\text {th }}$ meeting, p. 1).

The next activity was to identify characteristics of 'the active participant'. This was done by writing and drawing on flipcharts by the group. Characteristics that were often mentioned were 'having an open heart' and an 'open view', willing to listen, observe and share opinions, more thinking and practising of what is learned, and be more active during training or workshops (see annexe 13 for an overview of the characteristics given by the group members). After the outcomes were discussed, the researcher provided a lecture on participation and participatory activities. For the next meeting, group members were asked to think of activities that could be included in the Training Guideline, using an activity form (annexe 16). 
During the fifth meeting, the activities the members prepared for this meeting were presented to each other. The group decided which ones would be included in the guideline. The members proved to be more critical than before:

One group member presented an activity that was specific for science education, and not a training activity. After he translated his activity in Khmer, the others said 'it was very specific and should not go in the Training Guideline'. (field notes $5^{\text {th }}$ meeting, p. 2).

The next activity was 'stepping through the implementation phase' in order to decide which steps should be included in a checklist for this second phase. The members worked in groups of three and by putting steps on 'post-it' notes. In the big group, the 'post-it' notes of the groups were mixed and put in order, thus creating the second phase checklist (annexe 15). This activity was chosen to expose the community of learners to an alternative methodology for making an inventory of approaches.

The sixth meeting started with an icebreaker, an energizer and a wrap-up activity. After the three activities were completed, the group was asked to decide which of the activities was the icebreaker, the energizer or the wrap-up. This activity was followed by a brainstorm activity on six flipcharts (two for each kind of activity) that were passed quickly between the members sitting in the circle in order to identify characteristics of these activities. The members were invited to write one idea on each chart that passed them. Such time pressure forced the group members to decide quickly. Previous experience on the part of the researcher showed that brainstorming activities in Cambodian organisations usually turns into group discussions. The time pressure was able to prevent that and enabled the group members to come to their own ideas. The results were discussed in the group, after which the researcher provided a short lecture on icebreakers, energizers and wrap-up activities, in order to enhance the members' conceptual framework by categorizing the different activities. Then the categorization was developed and agreed upon by the group (annexe 17). Next, the steps of the first two checklists were reconsidered, leading to the final checklists (annexe 18).

The organisation and conduct of the energizer at the beginning of the seventh meeting was given to one of the group members, in order to pass on more and more responsibility from the researcher/facilitator to the group members. The 
next activity was designed to find the definitions for monitoring, evaluation and follow-up that were used by the different Departments and the DGE at that time. Definitions and key words were written on pieces of paper, and then attached to the three flipcharts (one for each process) on the wall. After this 'focus' activity, the researcher conducted a lecture about the three processes, in order to strengthen the conceptual understanding for these three processes. During the final activity of this meeting, the group developed the first prototypes of definitions for the three processes (annexe 19).

Meeting eight started with a presentation of the definitions of monitoring, evaluation and follow-up that had been developed during the previous meeting. After each definition, the group thought about how each process could best be conducted. By then, the group was actively debating how the definitions should be formulated, in an open and collaborative way. Suggestions were accepted or dismissed by the group:

They were all standing around the table, and everybody was proposing key words and formulations to the member writing down the definitions' (field notes $8^{\text {th }}$ meeting).

When all members accepted the formulations, everybody started to write them down in their notebooks because, as one of the members said 'Now we agree on this altogether, so we have to note it to be sure (we all keep the same formulations)'. After the group had decided on the definition of 'follow-up' and how it could be done, the researcher presented the four training components model of Joyce and Showers (2002) for effective learning in professional development endeavours, in order to stress the importance of follow-up to boost learning. Last, the group thought about a checklist for reporting.

Meeting nine was a 'special participatory workshop' held outside the Ministry, in the meeting room of a health sector non-governmental organisation (NGO) in Phnom Penh (HealthNet/TPO), because this room had the facilities for conducting state-of-the-art participatory activities. It was also anticipated by the researcher that a place where the group members could experiment with participatory activities without being interrupted would be beneficial to the learners. This meeting is discussed more elaborately in the next section. 
At the beginning of the tenth meeting the group discussed the special participatory meeting. All agreed on the relevance of the workshop for the strengthening of their facilitation skills. One group member said in the final interviews: "That meeting was good because some activities, the games and the problem tree were useful'. The next activity involved further improvements of the definitions for monitoring, evaluation and follow-up. It was also further explored how, when and by whom these processes should be conducted. This led to the third prototypical definitions and process descriptions (annexe 20). The next activity involved the identification of useful techniques for group discussion. During the special participatory meeting group members had indicated that they would like to learn more techniques for this form of discussion. Three group discussion activities were discussed and it was decided they would be included in the Training Guideline. All of them had been used at least once during the previous meetings. The last activity of this meeting was a final check of the shortterm evaluation form, as the test at the end of the special meeting showed that two questions were difficult to answer. This led to the final, slightly adapted version of the evaluation forms. The first draft of the Training Guideline was provided to the group members in order to read it and provide feedback.

The eleventh and last meeting of the working group focussed on the draft Training Guideline document. The first activity was to collaboratively develop an overview of what should be included in the guideline document. The next activity was to identify feedback comments on the draft Training Guideline document, by asking the group members to write each comment on a different 'post-it' note. Next, the 'post-it' notes were categorized and the main issues were discussed first. The main content mentioned by the group was about when to propose the training or workshop to donors and how to report afterwards. Methodology was neglected. After some probing by the researcher, the group added content regarding the role of the facilitator, and methodologies for group discussion and training games. This participatory approach was chosen to make as many improvements as possible in the limited time available. Usually, this kind of documents is discussed page-by-page, leading to inefficient and lengthy revision periods. The approach adopted during this meeting introduced the group members to another way for revising documents. The checklists, evaluation forms and activities were all looked at once more, before everybody agreed on the final version. 
The special participatory workshop

The special participatory techniques workshop was organised because it was felt that the group needed to experience a series of participatory activities in an optimal setting. This seems to be at odds with taking 'where people are' in their development as the starting point, instead of 'how it should be', but it gave the group members the necessary framework as to how participatory techniques could also be used. The location outside the Ministry was deliberately chosen to give this meeting a special status. Firstly, it aimed at providing the working group members with the experience of using participatory activities in an optimal environment. Secondly, it was hoped that the benefits of not being interrupted would become apparent, as Department staff often interrupted meetings by coming in and asking for signatures from the more senior group members. Thirdly, it was hoped that people would be able to act more freely in a different environment. Each member invited at least one other staff member of his or her Department to join this workshop to learn how to make professional development activities more participatory. The room was well-equipped for such activities as it was a big room, with carpeting on the floor and tables and chairs that were easy to rearrange in different configurations.

\subsubsection{The preparatory meetings}

Six months after the last working group meeting, three preparatory meetings were scheduled for February and March 2008, in order to organise the four-day Training Guideline training planned for April 2008. The three preparatory meetings were planned over a two-month period, in order to allow group members enough time to prepare in advance. In reality, however, almost the entire planning for the Training Guideline training occurred during the third meeting, resulting in the researcher providing more support to the group members than anticipated. This was for several reasons: One, the translation of the Training Guideline document in Khmer was not finished before the third meeting. Two, the group member responsible for the planning did not join the first two preparatory meetings, and, as a consequence, although those group members present were motivated, the group was not able to come to actual decisions:

The discussion was going in every direction, until the person who held responsibility came in during the last five minutes of the meeting. That was when the decisions were made' (field notes $1^{\text {st }}$ preparatory meeting, $p .2$ ). 
Hierarchy seems to strongly influence decision-making. When all group members who were to be the facilitators of the training were present at the third preparatory meeting, a week before the start of the four-day training course, they were able to accomplish their goals. During this meeting the training schedule was finalized and facilitators' names allocated to the sessions of the training (annexe 21). The checklist for preparing PDAs included in the guideline was used. At the end of the meeting, four of the group members asked the researcher to discuss their sessions in the week before the training started.

Three, despite the assumption of the researcher that a prolonged preparation time would lead to a collaboratively well-planned training endeavour, the time provided was not used effectively for collaborative preparation. There were two main reasons cited by the group members for not having used the preparation time. The first reason was the unfamiliarity with planning in general. Much preparation work in MoEYS is done on a last minute basis as a result of the overstretched agendas of staff members. The person responsible for the Training Guideline, for example, was given this task on top of his already overstretched job responsibilities. He asked the researcher repeatedly for help with his time management, but was never able to arrange time to discuss this. Secondly, planning assumes future thinking, a concept that was long denied to Cambodians, as explained in Chapter Three.

The second reason for not using the time provided was that the participants probably did not see the advantage of collaborative development of such an endeavour as training, in relation to the time it takes. People are used to developing training sessions on their own and usually at short notice. By doing it that way, they can organise their session when it suits them. The downside of this approach is not yet acknowledged, leading to overlaps in sessions, and dependency on the same training techniques, instead of a rich mixture. For example, one of the facilitators of the Training Guideline training started his sessions on the third training day by giving all the definitions that had already been presented during previous sessions. Another example showed the overlap in methodology for brainstorming with 'post-it' notes during two succeeding sessions, although multiple brainstorming methodologies were used during the working group meetings.

During the second preparatory meeting some staff members showed signs of irritation towards some colleagues caused by their high absenteeism and 
tardiness. On one occasion, it led to a confrontation between group members that could still be seen (by the researcher) as a polite rebuke, but in fact was quite a strong confrontation by Cambodian standards:

Two members said, still politely smiling, that they 'understood the busy schedule (of the group member responsible) but that (they) needed time to prepare too.' Then they asked the question 'when the guideline could be finished in Khmer'. The group member responsible stared down at the table and softly said that it would be finished very soon (field notes $3^{\text {rd }}$ preparatory meetings, $p$. 3).

The strength of the confrontation is not explicit but is hidden in its unusual nature as such frustrations are not normally expressed. This shows that for at least some group members, the behaviour of others has consequences for their work (for example preparation) that they usually will not express, but with which they are not satisfied. Only when the frustration reaches a high-enough level, might some express their feelings. It could also imply that many do experience the same frustrations, but are less prepared to express them. Although coping with emotions like frustration is a cultural issue and not easily changed, these findings increase the obligation to create safe learning environments, where people do not meet such frustrations, or are able to cope with them.

One of the group members already knew during the preparatory meetings that he would probably be absent during the four-day training course. He did not say that to the researcher and the other group members. Instead, he made sure that he was given only minor activities to facilitate:

He (the working group member) signed up for icebreakers, wrap-ups and energizers, but not big sessions on facilitation or planning. Then he asked if the training could be rescheduled to another week, as he 'was asked to join a big donor-funded project' in the week the training was scheduled (field notes $2^{\text {nd }}$ preparatory meeting, p. 5).

What can be learned from this event is that sometimes there is reluctance to say that one cannot be present. This could be grounded in uncertainty avoidance, or fear of angering or disappointing the researcher and others. The project the group member was engaged in instead was donor-funded and provided a 'per diem' payment. He said that he needed to 'go there because it delivered future possibilities with the donor'. It can be concluded that such donor-funded 
projects give financial benefit to Ministry staff, but also that other work might be postponed or cancelled in favour of it (in this case facilitating sessions at the Training Guideline training). The other group members were not willing to reschedule the course, because following the celebrations of Khmer New Year immediately after the dates of the training, MoEYS would start to prepare for the central secondary and high school exams, and thus staff would not have the time to join a four-day course. One of the group members said to him:' It will be very difficult to change these dates, because then other people will be away on those other dates'.

\subsubsection{The four-day training course}

The four-day training course was a success, especially when comparing the group members' learning progress with their starting points as facilitators. The core of the working group had made huge progress in the facilitation skills they used and their understanding of the background knowledge involved. Two members of the working group were absent because of other duties.

The difference in progress between the three group members that had been present during almost all working group meetings and those that had not, was striking. Of the facilitators who were not often present during the group meetings, two were experienced enough to provide lectures and do some 'new style' small group discussions, with 'post-it' notes and flipcharts. One facilitator who was not able to do so had limited training experience and did not ask for any support from the researcher until the morning of the day he had to deliver his session. The three facilitators belonging to the core working group, on the other hand, provided sessions that were well balanced and included the provision of background information, participatory and learner-based activities, and a wrapup. Examples of activities were role-plays, problem trees, brainstorm activities and decision-making activities.

It was interesting to see what happened with the training games, such as icebreakers and energizers. Saying how important something is, like doing such games, does not mean that they will be done. During interviews and informal conversations, all group members had stressed the importance of these games. Yet, when it came to applying these games, many tried to avoid doing it and came up with different avoidance strategies. For example, during the allocation 
of the activities to the facilitators, the older and more senior members tried to give all the games to the younger and less senior staff:

It was not really discussed who should take over the training games (from the group member that was unable to come), but the two more senior group members decided that the younger group member should do the games (field notes $3^{\text {rd }}$ preparatory meeting, $p$. 1).

One of the older group members said that 'Older group members feel that they do not need this kind of activity'. This might be caused by the cultural importance of status. Games might be seen as trivial, and thus have a negative effect on status. At the training course, one of the facilitators with high status tried to evade the games, by asking if 'any of the participants knew a song or story' that could function as an energizer, while he or she was supposed to introduce a game. Another strategy for avoiding actual application was not to show up, as for example:

He (the group member responsible for conducting the energizer) was not there while he was scheduled to do an energizer during the morning of the second training day of the Training Guideline training, without notifying anyone (field notes $2^{\text {nd }}$ training day, p. 1).

Another example was to decline when another member offered to switch energizers, in order to allow the other group member to be absent the next day, but still perform his energizer today:

One group member said (to the researcher) that he was not able to perform the afternoon energizer because of other obligations. Another group member overheard that, and made a proposal to switch their energizers. He could do this afternoon's energizer, and the group member that was unable to conduct the energizer this afternoon, could do that the following morning instead. The first group member smiled a little, shook his head and said 'no, no'. (field notes $2^{\text {nd }}$ training day, p. 3).

Such avoidance could be based on fear of doing something that is unusual and could be seen as 'strange' by participants. 
After the four-day training, the group members received a certification letter, confirming their participation in the working group responsible for developing the Training Guideline and conducting the Training Guideline training (annexe 22).

\subsection{LEARNING DURING THE SCENARIO}

\subsubsection{Steps in learning}

The four training components model (Joyce \& Showers, 2002) was used to shape the overall intervention, but not in a rigid manner. Theory was provided to the group members, but only at appropriate moments in the micro-cycles. The researcher modelled behaviour during the whole intervention, ensuring a 'practise-what-you-preach' approach. As often as possible, the researcher provided meta-comments on what he did during or after facilitation. An example of modelling and articulating meta-comments was to explain the researcher's position in the room, the use of non-verbal communication and the utilization of his voice while presenting a PowerPoint presentation.

Practice was ensured during the meetings of the working group and, ultimately, during the four-day training course. Some group members experimented with acquired knowledge and skills during PDAs that were organised outside the scenario, and were supported by the researcher at the learners' request. Followup was provided to the group members during the working group meetings, the preparatory meetings, the Training Guideline training when necessary, and on other occasions upon their request.

Although the intention of the researcher was that peers would provide follow-up support to each other, in reality it was the researcher that gave most of the follow up. The group members did not see the necessity of having facilitator meetings, for example during the four-day training course:

Instead of meeting at the end of this first day, all facilitators left (field notes $1^{\text {st }}$ training day, p. 13).

Furthermore, it was not common to invite other group members to PDAs delivered by a colleague, and discuss the PDA afterward. The lack of peer followup remained a lost opportunity for learning during the whole scenario. This was 
partly because the participants did not see the importance of follow-up, but the main reason should be sought in cultural dispositions. In general, saying anything negative is uncommon in Cambodian culture. Furthermore, hierarchy prevents a less senior staff member from giving feedback to a more superior colleague, even if it was in the form of follow-up support. During the final interviews, the researcher asked one of the group members why she did not give feedback to her colleague. She answered: 'Oh, I could never do that; that is our culture.' This is confirmed by the observations that the only time a less superior officer gave feedback to a more senior colleague was during the confrontation described earlier.

It was found that comments given by the researcher as a foreigner were experienced as less threatening and more easily accepted, as the foreigner is an outsider. When during the third meeting of the working group one of the groups working on the evaluation forms could not come to an agreement on how to formulate a question

Two of the group members called for the researcher and asked who was right (field notes $3^{\text {rd }}$ group meeting, p. 1).

It could also be that the follow-up provided by the foreigner is found to be of 'higher quality', than that of local colleagues, or that the status of the foreigner is perceived as being higher.

\subsubsection{Taking learning a step further}

The collaborative learning approach used in the scenario was combined with the notion of 'learning by designing' from the corporate curriculum. Each step of learning was informed by the previous step, but also by what the learners had not yet grasped. In that sense, learners worked within their 'zones of proximal development' (Vygotsky, 1978). By bringing learners into situations of 'functional instability', learning was endorsed. One example is that of the activity in which the group members were invited to 'walk through' the steps of organising a PDA described earlier. The activity itself was unknown to the learners, but also asked them to question their own beliefs on as to how a PDA should be organised, as the position of steps that had seemed logical before were now being questioned. The outcome of the activity informed the researcher as to how to continue with 
the next step. The 'stepping' activity was the beginning of one of many microcycles characteristic for this scenario. These micro-cycles are explained below.

\subsubsection{The importance of micro-cycles}

Micro-cycles were used in a variety of situations leading to many important outcomes, for example checklists for the three stages of organizing PDA's (before, during and after), evaluation forms, and strengthened facilitation skills. The previous example was also the beginning of one of the five micro-cycles described in this section. These micro-cycles were essential for the 'fine-tuning' of the scenario. The first step of this first example has already been explained and showed how initial consensus was found for the checklists for organizing PDAs. The first exercise delivered an initial prototype. During the next meeting, the steps were laid out and 'walked through' again. Changes in the succession of steps led to a second prototype. During the third meeting the steps were put in a checklist that was tested and adjusted, leading to the final product (annexes 14, 15 \& 18).

Another example was how micro-cycles led to the construction of evaluation forms. The working group produced the evaluation forms during three meetings, by developing, revising and adapting the initial prototype. Advanced insights came from discussions and tests. The group members first discussed what evaluation forms looked like in their Departments, were then shown more advanced examples of forms and, when asked to collectively develop forms, were given the five critical levels of professional development evaluation model (Guskey, 2000). The model was found to accommodate the group members in their need to make forms that were academically sound. The academic background was provided at the appropriate stage in the iterative cycle the group was going through (see the progression of the evaluation forms in annexes 8-11).

A third example of a micro-cycle is the professional development of the individual working group members. During the meetings methodologies were explored and discussed. The methodologies were then tested during workshops and training endeavours which individual group members conducted outside the working group. This testing, it must be said, was the most difficult step in the implementation of the intervention, as time constraints were manifold and appropriate training endeavours scarce. In some cases, however, this worked well. For example, one member adjusted one of the methodologies used in the 
working group for a workshop in her Department. After the workshop, she evaluated the methodology and how she had used it, and adjusted it for a second workshop. She thus became more 'artisan' in the use of the methodology. She presented her experiences in the working group, and the methodology was then included in the Training Guideline.

Another example of a micro-cycle is how understanding language issues informed the 'fine-tuning' of the scenario. It was found that the English wording in handouts and PowerPoint presentations was often too difficult for some of the group members. Easier wording and also using Khmer for these difficult English terms improved communication and learning. For example, words like 'previous' were replaced by 'before' or 'last'.

The final example presented here explains how 'fine-tuning' led to the formulation of the definitions for 'monitoring', 'evaluation' and 'follow-up'. During the seventh meeting, working group members were asked how they used the terms, using a brainstorm technique in which people were asked to write their ideas on the upper side of each flipchart (one for each term). Then strips of paper with academic definitions were given to the group, in order to discuss and decide which definitions belonged to which terms. When the group came to a decision on each definition, they were asked to attach the definition in the middle band of the appropriate flipchart. The next step was to decide on the 'group definition' for each of the terms and write those in the lower band of the flipchart. Then another flipchart was put over each of the written flipcharts, to brainstorm about who would be responsible for each of the processes, how that should be done, and when the process should take place. The eighth meeting was used to discuss and further develop the definitions, and decide on the 'who', 'how' and 'what' for each of the processes. The next step was to translate the definitions into Khmer, after which the group members were asked to take the definitions back to their Departments and ask for feedback. The final step, during the next meeting, was to come to a consensus on all three definitions (annexe 20).

The descriptions of the micro-cycles show that 'where the learners were' was taken as the starting point of each cycle, in order to ensure relevant learning and products. In many cases, like the evaluation forms and the definitions, both the products and the learning were high-level and beneficial for the group members. In some cases, the outcomes were less advanced, but at least did not push the 
group members to develop materials that would be too highly advanced to use. One such example is the 'preparatory checklist' presented in the Training Guideline (Berkvens, 2007b). This checklist shows two items (item 14 and 17, see annexe 14) that are separated, but do belong together.

\subsubsection{Strengthening meta-cognitive, regulative and communication skills}

As mentioned earlier, the researcher tried to gradually hand over meta-cognitive and regulative skills to the learners during the intervention, by letting the learners take more and more responsibility for their learning. For example, in the beginning the researcher prepared all activities and led the participants through them, but at a later stage initiated activities by letting the learners facilitate sessions during the working group meetings. Using many participatory activities in which the learners acted together strengthened communication skills. Handing over meta-cognitive, regulative and communicative skills remained a difficult process throughout the intervention. It is fair to conclude that cultural and historical backgrounds still have a strong influence on these skills today. This also applies to teacher reliance. Although the researcher tried to hand over learning control, the learners remained strongly dependent on the researcher during the entire scenario. Ironically, the observations of the Training Guideline training show that such teacher reliance is no longer necessary for most of the group members as they are able to facilitate sessions by themselves. For example, the two core members provided wellbalanced and participatory sessions, but still asked the researcher for confirmation on the quality of their sessions. Their facilitation skills and background knowledge were strong enough to act independently. Here again, uncertainty avoidance and the fear of making mistakes seem to play an important role in this dependency. As mentioned earlier, meta-cognitive skills are very weak, including planning, critical thinking, reflection, following plans and time management.

\subsubsection{Conclusion}

The process that delivered the enacted scenario provided interesting insights on how the context and culture of Cambodia influences learning. The adoption of collaborative and 'learning by designing' approaches based on corporate curriculum and socio-constructivist ideas led to more contextualised definitions, concepts and methodologies. Incorporation of the four training components of Joyce and Showers (2002) led to more application by the learners. 
It was not only the working group members who learned during the scenario, the researcher learned about the contextual possibilities and impossibilities too. For example, the (academic) background information that was provided to the learners was compared with the Cambodian reality, thus creating a mutual learning situation, in which the learners gained academic knowledge, and the researcher learned to understand the strengths and limitations of the Cambodian context. In this way strategies and tools were developed that were academically grounded, but also practical in the Cambodian context. The micro-cycles played an important role in the learning process.

The researcher provided support to the learners during the complete scenario. Although it was the intention only to observe the four-day Training Guideline training, and not really to provide support, nonetheless, support was requested continuously. With hindsight, the support provided could be seen as follow-up, since peer support was largely missing. On the other hand, it could also be that it was the learners' insecurity that led to such a high request for support; this would be consistent with the high uncertainty avoidance levels of many Cambodians. 


\section{CHAPTER 6}

\section{Evaluation of the enacted scenario}

\subsection{INTRODUCTION}

This chapter describes the evaluation of the enacted scenario. Figure 6.1 shows where the evaluation phase occurred (shaded) in the educational design research described in this dissertation. The evaluation is based on data that were collected during the design and implementation of the scenario. The data are analysed using the curricular spider web (van den Akker, 2003), the critical levels of professional development evaluation (Guskey, 2000), and the 5-D model for cultural differences (Hofstede, 1986; Hofstede \& McCrae, 2004; Gervedink Nijhuis, 2008).

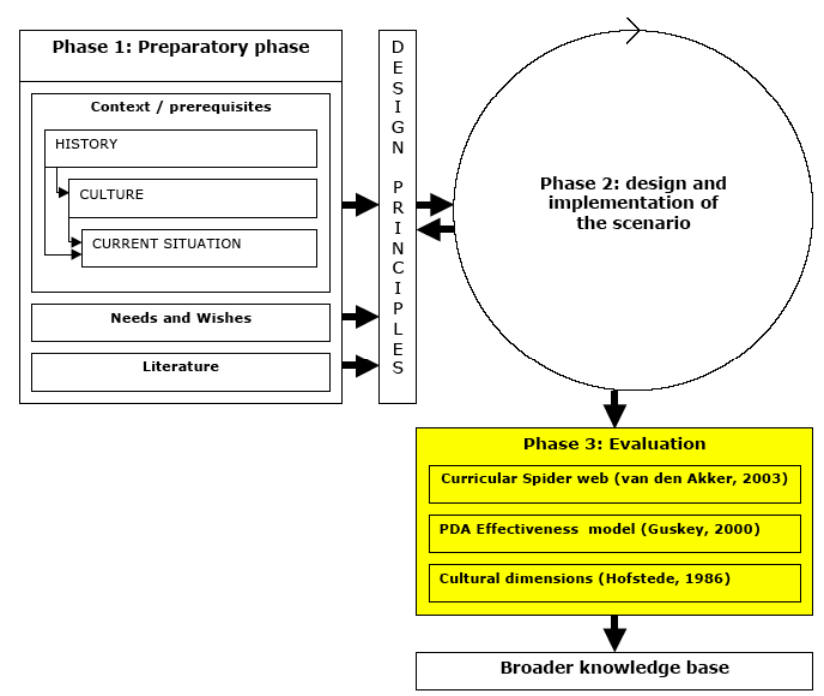

Figure 6.1 Overview of the evaluation phase 


\subsection{INSTRUMENTS AND PROCEDURES}

Data were collected at designated moments as well as on a continuous basis. The researcher observed all working group meetings, preparatory meetings and most of the Training Guideline training sessions. Informal conversations relating to the research that occurred spontaneously were also included as data. The group members filled out evaluation forms at the end of the last working group meeting and the researcher conducted final interviews with the group members six months after the last working group meeting.

\section{Observations}

The observations of the eleven working group meetings and the three preparatory meetings were conducted while the researcher was facilitating the meetings. This role mixture was not easy and demanded measures to prevent role bias and to secure thorough data gathering. This was done by making short notes of observed behaviour and events during the meetings that were elaborated upon as field notes immediately after the meetings. If necessary, the researcher liaised with the working group members to verify observations. Initially, an observer guide was developed, but was soon found to be too limiting. Therefore, the decision was made to observe as thoroughly as possible. The focus was on knowledge and skills application, behaviour that was stimulating or inhibiting learning following the 5-D model for cultural differences (Hofstede, 1986; Hofstede \& McCrae, 2004; Gervedink Nijhuis, 2008), and how collaborative, participatory and learner-based activities were received.

At the Training Guideline training, the researcher was present as an observer during the first three days. Thorough field notes of these observations were also written up in detail during each training day. The last training day could not be observed by the researcher, but was observed by one of the group members. The observations were later discussed with the researcher.

\section{Final interviews}

The final interviews took place six months after the last working group meeting. All group members were interviewed. The final interviews were deliberately scheduled to take place after such an interval in order to measure application levels of the acquired knowledge and skills by the group members. A semistructured interview method was used (Annexe 6). The researcher taped and transcribed the final interviews. 


\section{Evaluation forms}

At the end of the last working group meeting, the researcher asked the working group members to fill out the short-term evaluation form (Annexe 10). Absent members completed the form a few days later. All members delivered their form to the researcher. The outcomes of the evaluation were used to ask for more details during the final interviews with the group members.

\section{Informal conversations and expert opinions}

Informal conversations with the group members and participants together with conversations with experts provided considerable insight that was helpful when interpreting the observations and interviews. Important findings from the conversations were noted in the research log and consulted on a regular basis.

\section{Data analysis}

The three academically established models described in Chapter Two were used to analyse the data, in order to evaluate the outcomes of the enacted scenario against the intended results. The data mainly consisted of the observations of actions and reactions of the working group members, and the interviews held with them. The curricular spider web of van den Akker (2003) was used to assess the relevance and curricular consistency of the scenario, the five critical levels of professional development model (Guskey, 2000) assessed the practicality and effectiveness of the scenario, and the 5-D model for cultural differences (Hofstede, 1986; Hofstede \& McCrae, 2004; Gervedink Nijhuis, 2008) was used to evaluate the practicality of the scenario and the influence of culture on learning in general.

\subsection{EVALUATION OF THE ENACTED SCENARIO AS A CURRICULUM}

\subsubsection{Rationale}

The rationale for the scenario was based on a clear mandate provided by the Ministry of Education, Youth and Sports (MoEYS). The Ministry wanted to strengthen the knowledge and skills of its staff about professional development and their ability to organize and conduct training endeavours and workshops. Furthermore, the Ministry wanted to identify ways in which more effective

professional development for its staff could be established. Key words that the Ministry used in the mandate were: a 'participatory approach' learner-based 
learning'. In consultation with the Deputy Director of the Directorate General of Education (DGE), notions of a corporate curriculum approach as presented by Kessels (1996) and a socio-constructivist learning approach were adopted for the working group. This led to a 'learning by designing' approach that pursued what the group members wanted and needed to learn in order to come to a shared model for effective facilitation of professional development activities (PDAs) in MoEYS. The direction of the learning process was thus led by the learning needs of the learners, with a strong emphasis on collaborative learning and sharing of knowledge and skills. For example, during the development of the checklists for the organisational phases for PDAs, the group members actively discussed and developed the checklists collaboratively, using the three phases model of Guskey (2000). These three phases (what to do before, during and after a PDA) were introduced to the group members the second time they worked on the checklist for the first phase, as the researcher found that the group members were not yet able to distinguish between the different phases of PDA organisation:

One of the group members wanted to add monitoring and feedback in the checklist for the organisation phase of PDAs (field notes $3^{\text {rd }}$ group meeting, $p .4$ ).

Another example was the development of a follow-up structure for professional development. The researcher observed that the group members were unclear about the necessity and methodology for (peer) follow-up:

One of the group members started translating what the researcher explained about follow-up, because the explanation given by the researcher in English was not clear enough for the group members. During the translation, he turned to the researcher and asked if monitoring and follow-up are the same thing or not. He said that for him 'they are the same' (field notes $7^{\text {th }}$ group meeting, p. 1).

During the next meeting, the group brainstormed on how peer follow-up could be conducted in the Departments, after the researcher explained the benefits of peer follow-up.

Furthermore, the training components model of Joyce and Showers (2002) was used to stimulate application of the acquired knowledge and skills in the professional development practice of the group members. The researcher modelled many activities that were later used by the group members. 
He (the facilitating group member) did a brainstorming activity using 'post-it' notes, which the researcher had used during one of the working group meetings (field notes $2^{\text {nd }}$ training day, $p .7$ ).

Practising with the acquired knowledge and skills was included during the meetings and application in daily work was stimulated and supported by the researcher.

\subsubsection{Aims and objectives}

The scenario aimed at strengthening the group members' ability to organise and conduct participatory and learner-based PDAs. As a secondary result of the scenario, the group developed a Training Guideline and evaluation forms. Therefore, the group members needed to acquire a strong conceptual framework on professional development, had to be introduced to a variety of participatory and learner-based activities and had to have enough opportunities to practise the acquired knowledge and skills before applying the new approaches and concepts in 'real life' settings, i.e. during the Training Guideline training sessions. The researcher introduced many methodologies and activities during the working group meetings, but the group members did not always use them when delivering sessions. Examples of energizers were 'the quest', in which pairs of group members needed to acquire a number of items from a list provided to them (annexe 23) as fast as they could. Other training games were, for example, 'the numbers game' and 'folding paper' from the '100 training games' book (Kroehnert, 1991).

At the first meeting of the working group, the overall goal was presented and discussed with the group members. At the beginning of each following meeting, the objectives were presented using PowerPoint and handouts. The group members appreciated this, as one participant said: 'The goals and objectives are linked, summarized and put in the broader perspective'. Another stressed the importance of shared, common goals: 'Common goals for effective training at central level are important.'

At the ninth meeting, the researcher deliberately chose not to present the objectives. Firstly, because presenting them would have compromised the start of the meeting which was designed to be a 'surprising opening', and secondly to see 
if it would make a difference to the participants. The opening was a success, but the objectives were missed, as one group member said at the end of the meeting: 'Maybe the objectives of the meeting were unclear, and need to be presented'. The final interviews show that the majority of the group members found setting goals and objectives important.

Further observations of the Training Guideline training showed that almost all group members started their sessions with presenting the objectives, as modelled by the researcher during the working group meetings:

She (the facilitating group member) started the session on communication skills with the presentation of a PowerPoint slide of the aims and objectives of the session (field notes $1^{\text {st }}$ training day, p. 5).

Modelling and providing meta-comments about the importance of providing the aims and objectives of sessions thus led to application by the group members.

\subsubsection{Content}

The content of the scenario focussed on two main areas: 1) how to organise, conduct, evaluate and support a PDA; and 2) how to be a good facilitator using participatory and learner-based activities.

During the collaborative work in the working group, the researcher found that the members of the various Departments were using different operationalisations for terms and concepts (for example, for monitoring, evaluation, follow-up, participation, group discussion, etc), and that definitions and concepts were used inappropriately. For example, one of the group members said, while discussing participatory approaches: 'We do not do participatory activities, because it is teacher-centred'. This is just one example of showing the need for a shared conceptual framework on professional development. In order to arrive at such a framework, the terms and concepts were discussed during the working group meetings. This led to a new set of operationalised terms and concepts that was agreed upon by the Departments (Annexe 20). The group members appreciated the developed framework and found it relevant. One group member said: 'Now we use the same words.' Another claimed: '...especially identifying what monitoring, evaluation, group discussion are, what the differences are, ... what the steps are, what do first, and what (do we do next) is helpful.' 
Although two of the more experienced group members said that the content was not new to them, they had never previously had a clear understanding of how the terminology and concepts were related. One of them said: 'But (now) we keep and put in order and provide clear definitions and processes'. Three of the least experienced group members said that the content was 'very useful', 'the content is the most important (for me): How to teach?' and that 'content is good, because we have the content step by step'. The framework was developed by the working group, and helped the group members to work more effectively with the concepts and definitions. For the younger and less experienced group members, the content was new and thus directly placed in the framework. One said: 'I now know about definitions like monitoring, evaluation, follow-up, goals and objectives.'

Another aspect of content is its contextual relevance. The working group felt strongly that content should always be contextually relevant. Group members had a number of strong examples of contextually irrelevant materials used during recent training sessions and workshops. One group member said that he felt that

"content should be of real life in Cambodia, not "in the USA..." because they (the participants) do not know where the USA is. We need to use the idea from Cambodia. Like this book (showing the manual for 'child to child' community teaching) uses the example from Indonesia and Japan. It also contains an example about robots in USA, but we do not have, so we have to adapt to real life in Cambodia'.

The material he showed was in Khmer and produced by UNESCO in 2005 (Unesco, 2005). Even materials developed within the Cambodian context need to be modified to be relevant to various target groups. The same group member said:

'I now use and change examples, because (for) participants from provinces and districts we have to find the real example in the real life'.

The researcher found another example while working on a different project in Cambodia. This example shows how widespread contextually irrelevant the content of training endeavours in Cambodia is, and that merely translating documents from other contexts, as is often done by non-governmental organisations (NGOs) and donors, does not suffice. In one of the exercises of a training manual for Cambodian health managers developed by a German consultant, participants are asked to decide what the text is about. The idea is 
that the text does not explicitly mention the subject, and people find that they need their experiences to interpret information and provide meaning and context. A short piece of the text is presented here:

'The family was comfortably seated under the fir tree eating Leberkuchen and singing carols in the heated room, while outside the church bells rang to call the people to Mass'.

The consultant expected that on reading this passage, the participants would understand immediately from that context that it was about Christmas, even though there was no mention of the word 'Christmas' once. What she overlooked, however, was that the vast majority of Cambodians are Buddhist, have never seen a fir tree, have no idea what Leberkuchen is, church bells do not exist in Cambodia and if people could, they would prefer to cool rooms down rather than heat them.

\subsubsection{Learning activities}

As described in the rationale of the curriculum, a collaborative 'learning by designing' (Lowyck, 2001) approach was adopted. The 'zones of proximal development' (Vygotsky, 1978) of the learners were used to scaffold learning, by advanced discussions in the group and the provision of relevant background information. The adoption of the training components model of Joyce and Showers (2002) ensured that possibilities to practise with newly acquired knowledge and skills existed and that such practice was supported by follow-up.

During the working group meetings, many learning activities and participatory methodologies were introduced, practised, tested and evaluated. Some activities were put aside because the group decided they were culturally inappropriate, too complicated, or not cost-effective. Other activities were adjusted before entering another cycle of being tested and evaluated before they were included in the Training Guideline. For example the methodology of 'the problem tree' was rewritten in order to reduce the amount of materials needed:

In the original description (of the activity), participants were invited to write their comments on 'post-it' notes. They (the group members) changed that to writing with markers on the flipchart, as markers are less expensive than 'postit' notes (field notes $9^{\text {th }}$ group meeting, $p .4$ ). 
The activities included can be categorised into three groups: 1) Training games (icebreakers and energizers); 2) Lecturing techniques; and 3) Discussion, decision-making and evaluation activities.

The majority of the group members liked the training games because, according to one of the group members 'they made me very active, and friendly with working with the other members of the working group'. Another group member said: 'before the energizer was not so good, not so effective. We just provided new theory, just to give the story. (That was) not effective, because it was not stand up and to make the ... what I say to refresh the brain.' One group member, however, said that he did not want to do games, as a participant or a trainee 'because I do not feel comfortable'. He also felt that the games were not appropriate for older trainees and that 'energizers and games are not important for the workplace, only warm up to focus the brain on the (other) activities'. This group member was observed to withdraw himself every time a physical training game was introduced, for example during an icebreaker of the first training day:

He (the group member) took himself away from the group and moved behind a cupboard during the activity (field notes $1^{\text {st }}$ training day, p. 2).

With brainteasers, however, the same group member was very engaged and enthusiastic:

He (the group member) was very keen to solve the brainteaser problem (field notes $9^{\text {th }}$ group meeting, $p .1$ ).

This behaviour is probably linked to status, as 'acting silly' would not be appropriate, but more intellectual games like 'join the dots' (Kroehnert, 1999) would. In this activity participants are asked to connect a number of dots by drawing a specific number of lines without taking the pen off the paper.

Lecturing activities received the least attention during the scenario, although directions, feedback and demonstration lectures were given. During lectures, considerations about material and media use and choice of physical position in front of the screen were articulated. The provision of the theoretical importance of and rules for effective PowerPoint presentations was observed to lead to effective PowerPoint presentations by the majority of the group members. Before 
the scenario started, PowerPoint slides were observed to be filled with text that was often too small to read and never contained pictures. Bullet points were never used. During the Training Guideline training, the PowerPoint slides prepared by the group members contained bullet points instead of texts, pictures were included, and an appropriate font size was used. One example is of the group member conducting a session on communication skills:

'Her (one of the group members facilitating) PowerPoint slides were bullet pointed and neatly arranged' (field notes $1^{\text {st }}$ training day, p. 5).

Another example is that of the group member presenting about facilitation:

'His PowerPoint looked well organized. It was well listed and clear' (field notes $2^{\text {nd }}$ training day, p. 6).

The one facilitator who still used text-filled slides and did not follow the rules for presenting, was the one who joined the group of facilitators later, but never attended meetings of the working group:

'He presented his PowerPoint presentation, which was filled with text, seated' (field notes $2^{\text {nd }}$ training day, p.8).

All the group members valued highly the discussion and sharing of information with each other, as these were mentioned six times during the final interviews. For example, one group member said - very enthusiastically - 'I like mostly group discussion because it is democratic decision (making)'. Another group member said: 'During activities with 'post-it' notes I can put my ideas myself, share with everybody and decide with each other'. Many of the members identified the advantages of sharing ideas. For example, two group members noted that the new participatory methodologies made it easier for participants to give their opinion: 'As you know, most people in Cambodia are very shy...so writing on paper so we can know (their ideas)' and: 'Everybody is happy they can speak with the open heart'. Brainstorming was also mentioned as an important activity, because 'it motivates people to share their ideas'.

The working group generally felt that participatory activities were more effective. One group member, for example, said: 'The participatory approach is more 
effective, because it is to argue... you do not have to say like this or that, or because it is not appropriate or not specific... it is an (open) argument between participants'. One group member related participation to collaborative problemsolving: '...we can solve (problems) together'. The core members of the working group included participatory activities in their sessions of the Training Guideline training, using role-plays, different ways of small group work and discussions, and other learner-based activities with flipcharts and 'post-it' notes.

Although the appreciation of participatory activities was widely shared, some participants did identify a few constraints. They pointed out that both time and budget are limiting factors for the use of participatory approaches (this will be discussed in more detail later). One group member also mentioned the risk of people excluding themselves during group assignments by turning silent. The group members themselves were not observed to behave in such a way during the working group meetings.

\subsubsection{The role of facilitator}

The researcher adopted the role of facilitator during the working group meetings, but the group members also facilitated some activities during the working group meetings. During the Training Guideline training, the group members acted as facilitators. One of the activities for the working group was to identify key characteristics and behaviour of effective facilitators which were then included in the guideline as a 'behaviour code' for facilitators during training and workshops.

\section{The researcher in the role of facilitator}

The previous chapter identified some of the multiple roles of the researcher. This chapter identifies two additional roles: a modelling and a supportive role. The modelling role was recognised by one of the group members, who said: 'The teacher is a model: I learned your (the researcher) attitude: introduce what you want, practise in the group and show results to each other'. The researcher aimed at motivating the group members to try out new techniques. One of the group members recalled the researcher 'pushed us not to answer the question but pushed to try the idea'.

The researcher also provided much support, mainly on request. The majority of the group members asked for support throughout the entire scenario, but most of 
the support was provided during the preparatory meetings and the Training Guideline training sessions. For example, the group member who was responsible for the session on participatory approaches asked the researcher to help him with developing his session. After a meeting in which the researcher supported the group member to develop the session, the group member asked for feedback on the changes he made and again for more feedback the day before he delivered his session.

The group members expected that the researcher would always have the role of the leader. During the first preparatory meeting, it was obvious that the group members were waiting for the researcher to take the lead:

One of the group members asked the researcher 'What will we do this meeting?' The researcher replied that the 'Training Guideline' training needed to be prepared. But the group members kept waiting for the researcher to start the meeting instead of taking that responsibility themselves (field notes $1^{\text {st }}$ preparatory meeting, $p .1$ ).

Such 'teacher reliance' was no longer desirable at this stage, but not always possible to prevent. It was thus difficult to pass on responsibility to the group members, but during the same meeting it was possible to let some of the group members take responsibility:

With some encouragement from the researcher, one of the group members took the lead, although she was slightly reluctant. But when the group member responsible for the Training Guideline entered at the end of the meeting, the others gave him the role of leader (field notes $1^{\text {st }}$ preparatory meeting, p. $1 \mathcal{E} 4$ ).

The fact that the ad hoc group leader relinquished her role upon the entrance of the designated group leader showed that a cultural power issue was at work, which explains the reluctance of the others to make decisions. Interestingly, even the designated leader continued to depend on the researcher by asking 'Is this good, what we did?' at the end of the meeting.

Group members in the role of facilitator

The different facilitator roles were discussed in and identified by the working group. The group members decided that facilitators are responsible for organising sessions, and conducting them. During group assignments and 
participatory activities, he or she should support the groups. One of the group members said that the role of the facilitator was to 'walk around and facilitate all groups'. Furthermore, the modelling role was of importance to the working group. Potential rules were discussed, and led to a 'code of conduct'. An example of a rule was that 'facilitators could not go for a break to smoke a cigarette while participants were engaging in group work'.

The process of defining facilitator roles was necessary to enable the working group members to apply facilitator roles in practice. They all adopted their supportive role, as described above. Furthermore, most of the colleagues were observed to follow the code of conduct that was agreed upon during the working group meetings. But one of the group members repeatedly did not follow the established rules for trainees and facilitators during the Training Guideline training:

He (the group member) gave the task of designing a number of statements in groups of four or five, said they had fifteen minutes to prepare, and left the room (field notes $1^{\text {st }}$ training day, $p .10$ ).

His phone rang, but he turned it off and apologized to the group (field notes $1^{\text {st }}$ training day, p. 3)

His phone rang, but this time he answered it and said that he was busy before he shut it down (field notes $2^{\text {nd }}$ training day, $p .10$ ).

Although this behaviour was against the rules the working group had agreed upon, it must be said that this behaviour is partly explained by a hierarchical cultural issue. Many senior officials demand that their staff members be contactable at all times. During an informal conversation the researcher had with a Cambodian colleague, the colleague said: 'Our bosses expect us to be available for their calls all times.'

Aside from these incidents, the atmosphere during the four training days of the Training Guideline training was pleasant, and the facilitators created good connections between themselves and the participants. People were observed to work cooperatively together, discussing, laughing and making decisions together. During group work the facilitators walked around continuously and provided support when needed. Almost all group members helped to facilitate during these activities, even when it was not their session: 
Three of the other group members were facilitating the group work belonging to the session of one of the other group members (field notes $1^{\text {st }}$ training day, $p .4$ ). We (researcher and group members) all helped him prepare the next exercise, while some of the group members also facilitated the group work that was going on during his (the responsible group member's) session (field notes $2^{\text {nd }}$ training day, p. 4).

The researcher had not observed such a collectivist mentality during any of the other training endeavours and workshops observed before the intervention started. It showed shared ownership over the developed PDA.

The core group members were found to have made the shift from 'educators' to 'facilitators', following the description of Malvicini (2006). Although non-core members occasionally tried to adopt more 'facilitator' roles, they frequently slipped back into their old 'educator' roles, using mainly lecturing and big group discussions as methodologies:

He was lecturing for 75 minutes straight, people became distracted (field notes $1^{\text {st }}$ training day, p. 4).

They had to discuss two questions and come to an outcome with the whole group (field notes $3^{\text {rd }}$ training day, p. 6).

Two of the core group members were found to be more 'artisan' as facilitators, meaning that they were able to adjust activities to different contexts:

She (one of the two 'artisan' facilitators) started with a simple energizer, then introduced what the participants were going to learn, provided theory and a small group assignment, followed by role-plays and a wrap-up (field notes $1^{\text {st }}$ training day, pp. 5-6).

He (one of the two 'artisan' facilitators) developed and conducted a well-balanced session, with an energizer, theory, participatory and learner-based activities and a wrap-up (field notes $2^{\text {nd }}$ training day, $p .11$ ).

\subsubsection{Materials}

Five different categories of materials that were used during the scenario can be identified: 1) (academic) background material on professional development and professional development activities; 2) learning materials that were used to make 
learning activities more effective; 3) media; 4) stationery; and 5) the developed Training Guideline document.

The first category included articles, summaries or presentations of academic background about professional development and facilitation. These materials were provided to the working group members during the meetings of the working group in order to stimulate their learning about these topics. Although the majority of the group members greatly appreciated these materials because they were found 'helpful', the majority of the members also said that they lacked time to read them all: 'The materials were very useful for me. I had handouts and books. But no time to read.' Therefore, the researcher included time to read or provided short lectures to allow the group members to acquire the theoretical background that was needed for following activities.

The second category involved descriptions of training games and other learning activities, including a copy of the '100 training games' book (Kroehnert, 1999). These materials were given at the request of the group members, as one of the group members asked if the researcher 'could provide more of these games', but also in order to equip the group members with a rich source of activities they could easily use when preparing training sessions or workshops. The working group members all appreciated the book. But one group member said that the English in the book was too difficult, although that he had found ways to be able to use the book anyway: 'It is difficult for me to use the game book. My English is not good enough. The book is not Khmer, sometimes I don't know but I use the idea. Sometimes I have to ask someone.'

The third category involved the use of media, like PowerPoint and handouts. The researcher used his PowerPoint presentations and the handouts as opportunities for teaching the group members how these media should be prepared and used appropriately. Sometimes flipcharts were used instead of PowerPoint to show group members that these could be as effective as PowerPoint, and that using flipcharts is also bound to rules of effectiveness. On one occasion handouts were not provided, leading to reduced attention from the group members:

Everybody was observed starting to make notes of what was presented, often asking others what to note (field notes $4^{\text {th }}$ group meeting, $p .1$ ). 
This behaviour was in contrast with when handouts were provided. Then group members did not take notes, or only made small comments on the handouts. As with the articles described earlier, the handouts were appreciated, but time to read them afterwards was limited. One of the group members said: 'Materials like handouts were good, because they provided the crucial (information)'.

During the Training Guideline training, all facilitators were observed to provide handouts to the participants. The handouts were usually handout-style prints of the PowerPoint presentations. The use of PowerPoint for presentations has been discussed in detail earlier in this chapter. One facilitator, though, had a very high-level PowerPoint presentation, and he shared his knowledge with one of his colleagues:

He (the facilitating group member) used a wonderful PowerPoint presentation, with points that were highlighted and then faded again when the next point was projected (field notes $1^{\text {st }}$ training day, p. 2).

He (another group member) used the same PowerPoint techniques (as the previous group member), but he could not do that before. When the researcher asked how he learned this, he said that the other group member taught him how to do that (field notes $2^{\text {nd }}$ training day, p. 5).

The fourth category of materials included stationery like 'post-it' notes, flipchart paper, markers, tape and 'blu-tack'. During the whole scenario these kinds of materials were available in sufficient quantity, but this was often not the case for other PDAs where group members were trying to use participatory activities. All participants mentioned such lack of materials and linked it to budgetary constraints. One example: 'I like the activities with 'post-it' notes because it is very active. But we have not enough material. It is good for you (with you as facilitator because you bring the materials), but we do not have the budget'. One participant explained the consequence of not having enough materials: 'If the Ministry does not provide money or materials, we change the other way: we teach them (the participants)', meaning a return to lecturing and group discussions. The introduction of participatory methodologies was a request of MoEYS, and it was learned that the Ministry was able to provide the necessary materials if group members prepared the request appropriately. What needed to change was that the 'standard' materials that were normally purchased for each participant attending the training (notebooks, pencil, highlighter and folder) were replaced by 'post-it' note pads and markers. 
The fifth group of materials included the Training Guideline document and tools developed during the working group meetings. In the second preparatory meeting, the members began to use the guideline and the checklists as tools to organise the training. This use was confirmed during the final interviews, as the majority of the group members said that they had started to use the guidelines, evaluation forms and checklists. The group members often used the document during the Training Guideline training:

He (the facilitating group member) projected the appropriate part of the document on the screen and explained about PDAs (field notes $1^{\text {st }}$ training day, p. 3).

He (another facilitating group member) referred to the Training Guideline document and explained (field notes $2^{\text {nd }}$ training day, $p .7$ ).

In addition, during informal follow-up meetings with the group members eight months after the conclusion of the research, the researcher observed that the document was still prominently placed on the desks, and the members said they used it regularly.

\subsubsection{Grouping}

The composition of the community of learners for age, gender, level of education and previous experience with training and workshops contributed to a balanced working group that was able to develop a scenario and deliver the products required by MoEYS. The more senior staff members provided important experiential input that guaranteed the practicality of the guideline, and the younger members were enthusiastic enough to motivate most of the others to engage in the more unfamiliar activities introduced during the working group meetings. For example:

One of the older group members said: 'This activity (a training game in which a small ball held in the neck of one participant was passed to the next) is too uncomfortable for older participants'. The younger group member, who had introduced the activity, said that 'the participants he worked with loved it'. In the end, the activity was put in the Training Guideline, but the group members agreed that it should be used in groups with younger participants only (field notes $7^{\text {th }}$ group meeting, $p .1$ ). 
It was found that the members of the working group started to contact each other regularly on issues outside the scenario. Most group members experienced this as an important beneficial outcome, as collaborative work in MoEYS was not customary previously. One group member said 'It is more easy now to communicate with other Departments', and another said: 'Now we have better communication between each other than before'.

An informal core group of five members established itself during the meetings of the working group. These members were usually present at the meetings, and tried to practise and experiment with the acquired knowledge and skills during the meetings and in their work. Three of the core members facilitated sessions at the Training Guideline training, the other two core members were unable to join the training due to other responsibilities. Three of the four non-core group members facilitated at the Training Guideline training. One other facilitator was added to the group of training facilitators, but he had no connection with the working group and presented only one session.

Grouping also encompasses how participants of PDAs should be grouped for activities. This was discussed during one of the working group meetings, and even more thoroughly at the preparatory meetings for the Training Guideline training. One group member said that 'It depends on the activity who we should put together (participants) with the same role or as an office or working group'. At this point the group members entered a debate on the influence of hierarchy, following the strong conviction of some group members that 'the "higher ranking officials" (are) not doing games and so on, they are just discussing at the table.' Some group members held the belief that staff should only receive training that is appropriate for them, and that different levels in the education system should receive different training. One group member said: 'We should provide different training for the district officers, than for the school directors, as they are only responsible for monitoring, not for the conduct in schools'.

During the Training Guideline training, all different kinds of grouping were used by the group members that facilitated sessions, depending on the activities. Assignments were prepared for individuals, groups of three to six people, or the whole group. At first, the participants of the Training Guideline training had difficulty with the changing group compositions: 
The facilitators had just put the seats in a new arrangement during the break, but the participants started to move chairs and tables back when they returned from their break (field notes $1^{\text {st }}$ training day, p. 9).

After a while, the participants became used to changing seating arrangements, and respected those. Participants joined the activities more and more freely, as the training continued:

(In the beginning) the participants remained seated, and were giggling nervously when they were put into groups to brainstorm and discuss the problem (field notes $1^{\text {st }}$ training day, $p .4$ ).

But by the second day:

The participants worked hard in their groups. They discussed intensely and prepared the presentations very seriously (field notes $2^{\text {nd }}$ training day, $p .7$ ).

\subsubsection{Location}

The meetings were originally intended to take place in the large and more suitable meeting room of the DGE, but were relocated to the small meeting room because the big meeting room needed to be kept available for high senior officials. If the working group would have had formal status, the meetings could have taken place in the room that was more suitable for participatory and learner-based activities. Group members said that they found the small meeting room at the DGE 'too narrow', 'too small', but on the other hand also 'quiet to discuss' and 'ok'. One member said that the big meeting room would have been more suitable, but that nobody in the group had the authority to claim the room. The small 'room' was more like a corridor between three offices, and not very suitable for participatory and learner-based activities. The room was not regularly cleaned and was filled with unused bookshelves and files. After the first meeting, however, most of the stored furniture and files were removed and the room got cleaned regularly.

By Western standards, neither of the two meeting rooms at the DGE, nor most of the meeting rooms in the Ministry, are optimal locations for participatory activities. Most meeting rooms have massive conference tables and chairs, 
limiting the space needed for many of the more 'mobile' activities. The meeting room, which was used for the special 'participatory' meeting of the working group, on the other hand, was well suited for participatory activities. It had carpet on the floor which allowed floor work, was big enough for various learner-based activities that required space, and offered enough wall space to attach the produced flipcharts to. This room was described by the group members as 'very suitable', 'a good room, with space (in which) we can do the activities', and 'big enough for activity, sit on the floor, discuss about something, also good for icebreaker and work together'. Such meeting rooms are unlikely to be established in the Ministry within the near future, but it at least gave the group members an idea of what optimal conditions for participatory and learnerbased activities could look like.

Although one participant used the layout of the MoEYS rooms as a reason why some participatory activities could not be done, observations showed that, in general, people did not seem to mind and went with the situation as it was:

Because there was not enough space, one of the groups worked on the steps in organizing PDAs at the table in the small meeting room, while the other group used the small desk of the researcher in the room next door (field notes $1^{\text {st }}$ group meeting, p. 1).

Another example was the energizer in which a group member needed to guess what drawing was put on his or her back by the others:

There was not really enough space in the room to work in two groups, so the members decided to work in one group (field notes $2^{\text {nd }}$ meeting, $p .1$ ).

These observations show that the way MoEYS rooms are laid out does not mean that participatory activities could not take place, but that preparation and adjustments to the activities are needed in advance.

\subsubsection{Time}

The time component was found to profoundly influence the scenario. The component can be separated into four subcomponents: 1) The overall time available for the scenario; 2) Being present and on time for meetings; 3) Taking the time to prepare sessions; and 4) Time management during meetings and sessions. 
The overall time available for the scenario

The last gathering of the planned meetings was cancelled because of time constraints with the majority of the group members. The objective of this meeting was to further discuss the Training Guideline document in a collaborative way. Instead, the researcher discussed the document with the group members on a one-on-one basis in the weeks following the cancelled meeting. The meetings usually lasted two to three hours and were scheduled on alternating days of the week every two weeks, either in the morning or afternoon.

Six of the working group members were satisfied with the scheduled time for the working group meetings. One member found that 'the time between meetings was too long', and he said that 'maybe meetings can next time be closer together'. The intervals between the meetings, however, were necessary to allow group members to discuss outcomes like definitions and checklists in their Departments, and test activities and approaches in training sessions and workshops they delivered. The intervals were also provided to allow the group members to read the background information that was provided during the scenario. Most group members, however, indicated that they lacked time to read all the information.

A six-month interval was planned between the last working group meeting and the Training Guideline training, in order to measure long-term retention and use of the acquired knowledge and skills. But in January and February the schedule of MoEYS was too tight to allow for the training to be conducted. Therefore, the training was conducted eight months after the last working group meeting. This interval was found to be 'too long' by both the group members and the researcher. The time provided for the preparation of the training during the three preparatory meetings was two months and found to be 'good' by the group members, but underused by the researcher. This will be discussed in more detail in the section 'taking the time to prepare sessions' below.

\section{Being present and on time}

Arriving ten to fifteen minutes late is customary in Cambodia. Almost all meetings of the working group and the preparatory meetings, however, started with a delay of twenty to thirty minutes. Usually group members were called at the starting time, to make sure people attended. On the other hand, meetings always ended on, or before time. In order to end on time, the schedules of all 
meetings were adjusted and activities shortened or cancelled. One group member said in the final interviews that she always wanted to join 'but sometimes had other meetings to attend to', although she 'preferred joining the working group'. During the Training Guideline training, all mornings and afternoons started on time, or not more than fifteen minutes late.

High absenteeism during training is a given in Government structures in Cambodia, and has severe consequences for the knowledge and skills acquisition of any professional development program. The difference in the scenario for professional development presented in this dissertation is the inclusion of collaboration, practice and support. Attendance is important, and giving the project a formal status would have helped to boost learning outcomes. The responsible group member confirmed this by saying: 'It is my fault, because I did not establish an official letter to be a formal group. If we had been a formal group, we could say we have to have a meeting today. That is how it works with a formal group. But this was now not a formal group, so it was very difficult'.

When group members were absent, it was sometimes for private, but in most cases work-related reasons. Often group members were called to join other MoEYS or donor meetings. A minority of the group members informed either the researcher or colleagues of their absence in advance. Usually these were the group members belonging to the core group. In general, however, group members tried to be present as much as possible. One of the group members said: 'When you sent the letter, everybody tried to join, even when everybody was busy we still made time to sit together'.

Taking the time to prepare

The time group members had available to prepare sessions was usually limited. Although the three preparatory meetings to develop the Training Guideline training were planned over an eight-week period to allow for shared and collaborative development of the Training Guideline training, the actual preparation started only a week before the training course was scheduled. Time constraints and absenteeism of the staff responsible were the most important reasons. Not using the scheduled time for preparation had consequences for the follow-up support that the researcher could provide to the group members. On the other hand, immediately after the third preparatory meeting, thus a week before the training, three of the core group members asked the researcher to help them with the preparation of their session: 
As soon as the meeting was over, the core group members came to the researcher one by one, to ask for an appointment to help them with the preparation of their sessions (field notes $3^{\text {rd }}$ preparation meeting, $p$. 2).

Time management

The researcher was responsible for keeping time during the working group meetings, but the facilitators themselves were responsible for respecting the time during the Training Guideline training. Time management during the Training Guideline training can only be described as extremely problematic. Although the first session of each training day started on time, following sessions drastically overran their allocated time. For example:

The break was planned at 3 PM, but at 3.12 PM the second group started with its presentation. The break started at 3.48 PM instead and was not shortened. The following session was (so they could end the day on time) (field notes $1^{\text {st }}$ training day, p. 7).

Just one session in the entire four days finished early. The result of these extended sessions was that the facilitators of following sessions had to adjust their time schedules, and in some cases even had to conduct their session on the following day. The deviations of the time schedule were decided upon by the facilitators themselves (not the researcher), and without collaborative consultation with colleagues. On at least two occasions the time schedule was adjusted to meet the personal needs of facilitators, although colleagues would have been able to conduct those sessions without having to change the schedule.

The most drastic example of changing schedules was the sudden decision of the designated leader to finish the morning of the second training day early:

The designated leader decided that the session of another facilitating group member could be done in twenty to thirty minutes, and said to the participants that the morning sessions would end at 11 AM instead of 11.30 AM. As a result, the facilitator of that session needed to continue for half an hour in the afternoon, and the facilitator of the next meeting had half an hour less for his session on learning activities (field notes $2^{\text {nd }}$ training day, $p .5$ ).

This led to a confrontation between two group members during which the member responsible tried to apportion the blame to the researcher: 
One of the group members started to discuss the time schedule with the group member responsible (who had just changed the end time and thus the other times of the sessions). The discussion was tense and in rapid Khmer. Then the group member responsible said 'sooa kaang nih', which means 'ask this side', while shaking his head in the direction of the researcher. With this gesture and comment, he tried to blame the researcher. When the researcher met the group member responsible ten minutes later in private during the break, the group member started to apologize and took the blame immediately. He said that he 'prepared badly and that he messed up the time schedule' (field notes $2^{\text {nd }}$ training day, p. 3 \& 5).

It is important to see that the blame is attributed to the researcher in the group in order to prevent loss of face in front of the group, but to the group member himself when in private with the researcher.

As the training program was not handed out to the participants, they were less aware of the problems that arose because of the ever-changing time schedule, but the deviations led to frustration of at least some facilitators, who articulated their frustration to the researcher, but not to their colleagues responsible for the deviations:

One of the group members came to sit next to the researcher and said that he (one of the other facilitators) 'was talking too long and too much. The researcher replied that it would be good to sit with all facilitators at the end of the day and that he could say that to the other facilitators then. The group member said that 'that would not be necessary' (field notes $2^{\text {nd }}$ training day, $p .3$ ).

This kind of behaviour is linked to uncertainty avoidance and hierarchical issues that will be discussed in detail later in this chapter.

\subsubsection{Assessment}

Learning outcomes were not formally assessed with pre- and post-tests, but by using observations of the Training Guideline training sessions and training and workshops that were conducted by group members outside the scenario. Furthermore, the perceived learning by group members was assessed during interviews, informal conversations and by the evaluation forms that were filled out at the end of the last working group meeting. 
What the group members learned, and to what extent they were able to use the acquired knowledge and skills will be discussed in the next section on 'effectiveness'.

\subsubsection{Consistency of the scenario}

The components of the scenario were partly consistent with one another. The rationale of the scenario was clearly focussed on the mandate given by MoEYS. The learning activities followed this mandate and were selected on their collaborative and participatory levels. Grouping included the establishment of a community of learners. The aims and objectives were met, as the Training Guideline, including the tools, was produced and the organisation and facilitation skills were strengthened, although with varying results among the group members (two members improved to 'artisan' levels of facilitation, three to the 'mechanic' level, and four to the 'experienced educator' level) (see Chapter Seven for the developed typology). The learning activities allowed for ongoing evolution of understanding by the group members (for example, the developed conceptual framework about professional development) and the quality of the products (see the different prototypes of, for example, the evaluation forms). The materials provided were consistent with the learning activities (stationery in the form of 'post-it' notes, markers, and flipcharts, but also the academic background information about monitoring, evaluation and follow-up). The time allocated to the participatory activities was appropriate, but absenteeism had a significant negative influence on the outcomes of the scenario and formed the weakest component of the scenario. The location was also observed to be unsuitable for the activities. Although the group members confirmed the unsuitability during the final interviews, they demonstrated to possess the flexibility to adjust activities to the limitations presented by the location.

\subsection{EFFECTIVENESS OF THE SCENARIO AS A PROFESSIONAL DEVELOPMENT ACTIVITY}

\subsubsection{Participants' reactions}

The members of the working group were positive about the scenario. In general, they said they liked working on the guideline, with the majority of the members 
saying that they 'enjoyed it'. When asked why they enjoyed it, the group members said that the main reasons for the positive evaluation were the acquisition and practice of new skills, and the sharing of ideas by working collaboratively. One group member said: 'we like the brainstorming activity, because it makes us share and learn together'. The observations of the working group meetings confirm that the group members were highly motivated to and involved in working collaboratively:

All group members were actively involved in and contributing to the collaborative learning process while standing around the central table. People were agreeing and disagreeing during constructive discussion, and finally everybody approved the sequence of steps (field notes $3^{\text {rd }}$ group meeting, p. 3).

At the same time, working together was not always easy for the group members. According to one of the members 'sometimes the discussions were difficult'.

To the surprise of the group members, the group potential was found to be augmented by sharing knowledge and skills. At the end of a brainstorming activity, when all the ideas were centrally collected, one of the group members whispered in amazement: 'That is a lot (of ideas)!' Another group member mentioned the 'team spirit' he found explicitly present in the working group: '...I like so much and especially the team sprit...the team spirit makes us agree altogether to the output.'

The working group meetings were evaluated as 'being useful' by about half of the group members. One of the group members said: 'It was very useful, for me but I think also for the others'. At the end of the special 'participatory workshop', the participants were enthusiastic and very motivated to use participatory activities in their work. All participants expressed their positive feelings about what was done and learned that afternoon. This observation was supported with remarks of two group members in the final interviews. They evaluated the workshop as effective. One said: 'I would use all I learned (here)'.

Group members who were involved in the preparatory meetings agreed together on the effort they had made. They were positive about the training schedule they had developed, and found that it was designed as a practical and participatory training. In reality, however, the progress that was made during these three 
meetings was minimal, and the result was not really a collaborative effort. The individual facilitators designed their sessions in isolation from each other and, as already was explained, asked the researcher for support in the days before the training started.

A positive side effect of the scenario was that group members started to communicate more easily with each other than before. Some group members mentioned that 'it is more easy to communicate with other Departments now. Before, we did not know them'. Another group member said that the working group on HIV/AIDS became 'better because we use the shared knowledge we got from the Training Guideline working group and know each other.'

\subsubsection{Participants' learning}

All working group members said they had learned a lot during the scenario. For example during the eighth meeting, at the end of the morning, one of the members said: 'What we are doing now is real capacity building. Until now (before the working group) we just sat together and did what we thought was best, but now we are really learning and also add from the experience of each other... It is not just theory, but integrating theory and practice. Before the working group we did not really understand'.

The learning mentioned by the group members can be divided into three categories: 1) facilitation skills; 2) organisation skills and 3) theoretical background.

\section{Facilitation skills}

Group members said training and facilitation skills were important acquired skills. The majority of the group members mentioned the training games like icebreakers and energizers as 'good'. They said that these activities 'make the participants not bored'. The majority of the group members said to have acquired 'new techniques for brainstorming, group discussion, problem-solving, and decision-making'. 'The need to provide examples during lectures' was also mentioned, as was the importance of non-verbal communication skills. One group member recalled the importance of 'using body communication and look at the eyes to everybody that joins'. 
Organisation skills

The group members said they had 'learned how to prepare a Training Guideline', and 'how to organize training according to the guideline'. The created stepwise model consisting of three different organisational phases, each with a checklist was found to be a clear structure for organizing training or workshops. One participant said: 'I think that I learned guidelines, I know the steps how to organize the workshop or training session', and another member recalled the three phases: 'I got more knowledge on preparing before, during and after PDA'.

Some group members mentioned the 'need to do a 'training needs assessment', and the 'need to develop clear objectives, activities and content'. 'How to organize group size for group assignments' was mentioned twice. They also mentioned that 'size of groups depends on the goals: sometimes small, sometimes big', but also that 'we should do five or six groups when we have thirty participants'. Half of the group mentioned how to organise evaluation and that they learned 'how to make the list of the evaluation', and that evaluation forms 'should be provided after the workshop'. Only one group member did not appear to have understood the concept of evaluation, stating 'I am still not clear on evaluation for management'.

Monitoring and (peer) follow-up were not mentioned by any of the group members in the final interviews.

\section{Theoretical background}

In relation to theory and understanding of the knowledge base, the vast majority of group members said they had acquired mainly 'monitoring and evaluation' background. About half of the members mentioned they had also acquired 'new training methodology for group discussion and new facilitation skills'. Only one group member made a remark that pointed in the direction of follow-up. The theoretical background was appreciated, as it helped 'developing the definitions and to distinguish between the different processes'. Most of the group members also said to inform their knowledge on how, when and where to conduct these processes. One group member said: 'What I learned is how to monitor, facilitate, evaluate and when we use it, where we use it and how'.

Although group members claimed to have learned a lot, this was not always supported by the observations: 
The group was asked to develop an overview of what content and which tools should be included in the guideline. The list that was produced was a standard table of contents of any Khmer report on a PDA. Although methodologies were mentioned, not much was included about learning processes and concepts (field notes $11^{\text {th }}$ group meeting, $p .1$ ).

As stated earlier, some of the methodology and approaches were not new for some of the older and more experienced group members, but these were never practised and structured as clearly as during the scenario. One participant said that 'the terminology and techniques were not new, but were never discussed in detail' and 'before were confusing'. Another said that 'these techniques were never practised before, only presented'. Yet another explained that 'some methodologies were finally provided with clear definitions and instructions'.

\subsubsection{Organisational support}

As initiators, the Director General (DG) and the Deputy Director of the DGE both supported the scenario. The two DGE group members of the working group confirmed the support of their senior officials. One of the members said: 'The DG motivates us to join the team activities and the working group. They advise us to work hard and revise what is important and work on the guideline'. The researcher also experienced this support. The Deputy Director always made time to discuss the progress of the working group and provided relevant input when the researcher asked for feedback. The Deputy Director also opened the four-day training course, a culturally-explicit show of support, and in his opening speech, stressed the necessity of a more participatory approach to professional development, and how the Training Guideline and the training course fitted the bigger picture of capacity building.

The support that was provided by the Department Directors was of great influence on the learning possibilities of the group members, but the amount and quality of the support differed. All Department Directors allowed their staff members to join the meetings of the working group, but some staff members could only go 'if there was time', while others were advised to join all meetings. A younger and less senior group member said that it was for the Director of the Department to decide if he could join, but after she allowed him to join she supported him in attending all meetings. The most advanced core members were strongly supported and enabled to join all meetings of the working group and the four-day Training 
Guideline training. They made progress because they experienced the whole scenario and were fully involved in the micro-cycles. The other members were called away to other meetings, or sent to the province for assignments and therefore experienced sometimes strongly fragmented learning cycles. Furthermore, the degree of support provided by the Departments strongly influenced the possibilities for dissemination of the acquired knowledge and skills in the Departments. Some Departments believed that 'having a document' would be enough to make professional development activities more effective. The amount of support influenced the extent to which group members were able to experiment with and practise the methodology outside the scenario. The more the group members were able to practise, the stronger their facilitation skills developed.

One group member said that the way the Director of the Department behaved during departmental meetings created a gap between what was said and what was done in practice regarding the adoption of participatory approaches. The member said: 'My Director still relies on long one-way speeches, instead of allowing more learner-based and participatory activities'.

\subsubsection{Participants' use}

Differences were discovered between what knowledge and skills group members intended to use and what they used in reality. Many working group members said during the final interviews that they intended to adopt participatory and learner-based activities in PDAs in the future. For example, one group member said 'I can say that if I have another training session I will do this, change and practise what we did', and another member: 'In the next workshop we will get some action and energizers'. They also wanted to disseminate what they learned during the working group meetings to their colleagues in the Departments. As one group member said: 'I need to spread everything with my staff, my Director also'. However, about half of the group members were not able to implement and disseminate the acquired knowledge and skills in their Departments yet. One member said: 'I asked my Director for (a training) to organize, but I did not yet get it' and: 'I want to teach them (Department staff), but I do not get the opportunity to do that'.

The lack of possibilities for application, however, is not only caused by the time constraints or weak support by the Departments, but also by cultural issues like 
uncertainty avoidance, shyness, and power differences. An example of that is given by the following comment by one of the most experienced members of the group: 'I want to do more energizers and many games, but I think I do not know how to do'. Another cause might be that knowledge and skills acquisition was not strong enough for the members to confidently turn those into practice. Occasionally, understanding of methodology was not strong enough, leading to poorly implemented activities. One group member said: 'sometimes I do not understand and then I just try', explaining why crucial parts of an exercise during one of his sessions during the Training Guideline training were poorly executed. But, despite the context of all the described characteristics like high uncertainty avoidance, fear of loss of face and hierarchical issues, the most successful members were willing to overcome these factors. It is extremely significant that they were willing to experiment and learn.

One example of the gap between intended and enacted use is that of the group member who previously had said that he liked the activities that allowed participants to give ideas by writing on notes, instead of speaking them out loud because of the cultural issue of people being shy:

His session was the opposite of what he had said before. He pointed to people who had to answer him. Some participants felt threatened and started to giggle or tried to evade questions. He was the member who said that he liked the writing exercises to give comments, as that is less threatening for people. But he chose a form himself that was far more threatening to the participants (field notes $3^{\text {rd }}$ training day, p. 6).

In this case, the cause of not putting ideas into practice might be influenced by uncertainty. Other group members mentioned lack of time, the absence of the Training Guideline document, and limited budget as the main reasons for not yet having been able to implement the acquired knowledge and skills.

But the researcher also observed much enacted use. It was the core members who were applying most of the acquired knowledge and skills during the Training Guideline training sessions and PDAs conducted outside the scenario. The one facilitator of the Training Guideline training that did not take part in any of the working group meetings was not found to use any other activities besides lecturing. 
The majority of the group members were using the contextualised terminology during discussions and the Training Guideline training:

She (the facilitating group member) explained about monitoring, evaluation and follow-up. She differentiated well between the three processes. She used the terminology as put in the guideline (field notes $1^{\text {st }}$ training day, $p .4$ ).

Another example:

He (the facilitating group member) was lecturing about how to develop SMART (an acronym for Specific, Measurable, Attainable, Realistic, Timely) objectives. He explained what the letters stand for and what the words mean, following the guideline (field notes $1^{\text {st }}$ training day, p. 9).

Application of methodology was more varied, with the core group members using more different methodologies and applying these more often. An example of methodology use was a session delivered during the Training Guideline training, by one of the core members on communication skills:

She (the facilitating group member) started the session with a brainstorm activity using different colours of 'post-it' notes in order to be able to form categories, followed by sharing activities based on the outcomes of the brainstorm, and finished with an informative wrap-up (field notes $1^{\text {st }}$ training day, pp. 7-8).

Another example is the half-day session conducted by another core member:

He (the facilitating group member) started with an energizer that was linked with the subject of the session and did small group work. He also did a 'real-life positioning game, where the participants were asked to take a position in the room reflecting their opinion on a question asked. He wrapped up the session by reflecting on what was done during that afternoon (field notes $2^{\text {nd }}$ training day, pp. 6-8).

In addition, three of the core group members included learner-based activities in training sessions and workshops they delivered outside the scenario. For example: 
She (the facilitator) provided a group work assignment for indicating vectors of Avian Influenza infection. The group members had to work on a village map and show how they would include schools in preventing the disease from spreading. After the workshop she noted that 'brainstorming and group work were sometimes difficult, because people until now never had to give their ideas. So we need to encourage them to participate. Because before everybody kept silent' (field notes AI workshop, p. 1).

About using one of the learned approaches during a workshop for school directors, the facilitating group member said that the participants liked the new approach, because 'I did not 'speak speak speak' (talk a lot) so they got bored...but did small exercise and energizer...they were happy' and 'I used brainstorming as well'. Another member performed an icebreaker during a home-based training program in one of the provinces:

The participants had to introduce themselves while throwing a ball. Some of the participants did not understand at first, but after the activity they said 'we can use this, we can do, it is easy' (field notes home-based training, p. 3).

These observations show that the core group members were able to apply the acquired knowledge and skills outside the scenario. They were also able to adjust their facilitation to the needs of the groups in a more 'artisan' way than the others:

He (the facilitating group member) noticed that the participants started to get bored during his session. He decided to introduce a game. When asked why he decided to change his session at that moment, he said: 'suddenly maybe because I thought they were getting sleepy, but also because I thought they had to learn more energizers' (field notes $2^{\text {nd }}$ training day, p. 9).

An unexpected outcome was the aforementioned continuing collaboration of the community of learners outside the scenario. The majority of the group members were involved in the preparation and conduct of a multiple-day training on 'Project Cycle Management' executed by the DGE. Unfortunately, the researcher was unable to observe this training course, and data came only from interviews with the separate group members. According to one of the group members, the steps for organising PDAs mentioned in the guideline and checklists were used to develop the course. The involved group members said independently from one another that a participatory approach was adopted, and that the knowledge 
and skills they had acquired during the working group meetings were used. 'We did training games, but also group discussion. And how to be a facilitator was presented.' Not all participatory activities went according to plan. One group member reported that 'some participants of the training made wrong use of definitions and concepts' and that 'in general some of the activities were not clear and needed to be explained (again)'.

The researcher provided follow-up support to the group members who tried to implement the acquired knowledge and skills during the Training Guideline training and in their daily practice. This follow-up support was mainly important for strengthening the confidence of the group members. Requests for support were formulated in ways such as: 'Is it ok, how I do? What do you think?' or 'It is my first time, I am not sure about the content'.

Follow-up, however, is generally not seen as a crucial part of learning endeavours, and is therefore often neglected. In contrast with the non-core members, the core group members regularly asked for support, and often did so well in advance, enabling them to accelerate effective application of their acquired knowledge and skills.

\subsubsection{Secondary beneficiaries}

The participants of the Training Guideline training enjoyed the training games (icebreakers and energizers):

The participants just loved the game. They were laughing together, there was a lot of hilarity (field notes $2^{\text {nd }}$ training day, $p .7$ ).

Dropout was low. During the first two days there were many questions when activities were introduced, but later the participants were convincingly engaged in the activities, debating fiercely, sharing ideas, and putting effort in the production of flipcharts for presentations. During the evaluation, participants said that they 'learned new things, but they also never had the complete overview before'. The long-term utilisation of acquired knowledge and skills by the secondary beneficiaries was not evaluated.

An important issue is that participatory activities not only rely on sharing knowledge and skills but also on sharing opinions. Opinions are only valuable 
when they make sense to the subject at hand. That was sometimes difficult. Some participants had problems with identifying the message of activities, and tended to discuss the examples or cases used to work with a methodology, instead of the methodology itself. Many of the remarks were thus 'beside the point':

The participants were asked to give comments on the role-play methodology. One of the participants commented on the necessity of behaving formally when visiting a high-ranking official (content of the role-play), instead of on the methodology (field notes $1^{\text {st }}$ training day, p. 8).

From the facilitators' point of view, the participants benefited from the training sessions. One of the facilitators said that 'this training was good, because the participants were interested and wanted to know everything', and another facilitator said that the participants enjoyed 'learning new knowledge and shared their experience'. The opinion of the facilitators is supported by many observations, of which the most remarkable one is that of an older participant who was Chief of Office in one of the Departments:

He (the older participant) was defending a wrong concept about evaluation, almost on the point of getting angry. When he suddenly grasped what the others were trying to tell him, his face broke into a smile. As the presenter of his group's work, he later gave a strong presentation in which he was observed to use the right wording and described how effective evaluation should be done.

\subsubsection{Overall effectiveness of the scenario}

On the whole, the majority of group members were positive about the scenario and their personal learning. They appreciated the collaborative approach and the development of a contextualised conceptual framework for professional development. They also appreciated the acquisition of skills for the organisation and facilitation of training sessions and workshops. The developed products were greatly appreciated by both the group members and the Ministry.

However, gaps were identified between what group members said they had learned during the scenario and the actual acquisition in practice. These gaps occurred with the non-core members and one of the core members. The gaps were a result of lack of time, limited support by the Department Directors, sometimes based on shyness and uncertainty, and possibly on insufficient 
learning. The other four core members were seen to apply acquired knowledge and skills during the activities of the scenario, or during training and workshops they conducted outside the scenario. The inclusion of all training components of the Joyce and Showers model (2002) was necessary for advanced application of acquired knowledge and skills by the learners. The core group members completed the scenario and were involved in most of the micro-cycles, and frequently asked for follow-up support. The non-core members were not able to follow the whole scenario, were often absent during the micro-cycle activities, and showed less acquisition of knowledge and skills in practice. In general, acquisition of meta-cognitive and academic skills was weak with all members. This will be discussed in detail in the next chapter.

\subsection{CULTURAL INFLUENCES ON THE SCENARIO}

\subsubsection{The first cultural dimension: collectivist versus individualist}

Trust is the main characteristic in the collectivist/individualist dimension. As explained in Chapter Three, Cambodian society is collectivist, but trust has been heavily violated in the recent past. Reluctance to share information and articulate opinions was abundantly observed and also mentioned as an important issue for the use of participatory activities. During the working group meetings, a relatively safe learning environment was developed, leading to a more and more open and direct exchange of ideas, opinions and information. The aforementioned 'group spirit' is a strong example of the collectivism that established over time. A similar development towards more openness and readiness to share was observed with the participants of the four-day Training Guideline training:

Group work became more and more really a group thing, with lively discussions and - at least some - collaborative decision making (field notes $3^{\text {rd }}$ training day, p. 6).

The creation of such a safe learning environment, on the one hand, was found to be crucial in order to generate trust among the group members but, on the other hand, continued to be influenced by cultural power issues that could not be solved. The influence of power is described in the next section. 
Collectivist group behaviour also reinforced the risk of 'Abilene paradoxes' and 'group think' (Cooke, 2001). These pitfalls of collaborative activities were often observed in order to prevent loss of face and conflict. One such example is the group member who said 'Sometimes I follow the group, but do my own idea (when facilitating)'.

Senior officials were not present during the Training Guideline training sessions. Group members said that if such senior officials had been present during the activities, participants would have been less willing to give their opinion openly. It is likely that the same would have applied to the working group meetings, in which now only members of the community of learners joined.

\subsubsection{The second cultural dimension: power distance}

Power issues, especially hierarchical issues, were abundant and did have a strong influence on learning processes during the entire scenario. During the working group meetings, the younger and less senior members were not always accepted as equal partners, and their comments were sometimes left unnoticed:

One of the less senior group members wanted the group to use the checklist in the Training Guideline as a tool for organising the training. He did not feel comfortable to say it aloud. Instead, he whispered it to the researcher, who encouraged him to say it to the others. He did, but his comment was not listened to (field notes $2^{\text {nd }}$ preparatory meeting, $p .6$ ).

The hierarchical dimension also existed between more senior members. On two occasions tension arose between group members, when a member higher in the hierarchy tried to endorse a decision of which others close in the hierarchy did not approve. That led to a still polite, but tension-laden discussion. Similar confrontations have been discussed previously in this dissertation. In most cases, the members looked at the researcher for support. It was not always easy to remain neutral, but in the end a compromise was always reached. For example, in a group discussion during the second preparatory meeting on how the tools of the Training Guideline document should be used in the Training Guideline training: 
One of the more senior group member insisted on explaining all the tools in a separate session instead of during the sessions in which the background to the tools would be presented. The other group members did not agree, but they were not able to convince the group member. They turned to the researcher to intervene. The researcher decided to ask open questions, like 'Why do we use tools?' and 'Why do you want to use it in the separate sessions?' and 'Why do you want to use it in one session on tools?' In the end the group decided to present and explain the tools in the separate sessions, when the background was explained. They also included practice sessions to work with the tools (field notes $1^{\text {st }}$ preparatory meeting, p.4).

In most cases, however, frustration was only shared with the researcher, not with the person at whom the anger was aimed:

When the group member responsible was absent during the meeting, one of the other group members said: 'Where is he? He should be here!' (field notes $2^{\text {nd }}$ preparatory meeting, p. 2).

On at least two occasions during the Training Guideline training, a facilitator who was high in the hierarchy made a mistake and said something that was in contradiction to the Training Guideline document. It was noticed by some of the other group members, but they said they were not in the position to say so. Instead, it was expected of the researcher to correct the mistakes:

When the facilitating group member made a mistake, one of the others came to the researcher and said: 'You should say this', or 'He says wrong (makes a mistake), you need to correct it'. When the researcher asked why the colleague would not say it him or herself, the group member said 'Oh, I cannot say that' (field notes $1^{\text {st }}$ training day, p. 4).

This is likely to be connected with both uncertainty avoidance and hierarchical issues.

Power issues also influenced the application of newly acquired knowledge and skills at the workplace, especially when it was tried by the younger and less senior group members. One said: 
Sometimes I try to tell them (about participatory or learner-based approach) but they do not believe me' and '(I was not able to change anything yet) because I did not have the opportunity. I am so young in my Department, that when I say, nobody listens to me because they do not trust what I say. That is because they are older and they have a lot of experience.'

Another member said: 'For me I want to change, but sometimes the leader and senior do not agree, because they think I have small knowledge'. But the issue also existed between high-ranking staff members. One group member said that it was hard to change things in the Department, because 'some people pretend to already have the knowledge'. This was supported by the comment of another group member, who found that 'some colleagues were not interested'. Both felt that they did not have the power to make them participate in finding more effective ways to conduct professional development activities.

A striking example of how important hierarchy is in MoEYS became clear during one of the role-plays at the Training Guideline training:

The members of the participant group were looking for a folder to put the pretend letter in. They also wanted to attach the right number of paper clips, but could not find the clips. During the role-play the participants playing the requesting staff member was extremely humble, looking downwards, speaking in a soft voice, and bowing all the time, towards the participants playing the secretary and the Director General (field notes $1^{\text {st }}$ training day, pp. 7-8).

Even in hypothetical role-play situations, people felt they had to prepare and behave as in reality.

\subsubsection{The third cultural dimension: uncertainty avoidance}

Although the scenario took place in a generally pleasant and cooperative atmosphere, uncertainty avoidance was found to be one of the major cultural influences of the learning processes in the scenario. To a certain extent, uncertainty avoidance was informed by power distance, but it encompassed much more than that. The most important forms were fear of losing face, shyness, seeking approval, and fear of anger. 
Fear of losing face was identified to be both an inhibiting and a motivating factor for participating in group work and sharing. One group member said that 'fear of losing face' was a reason why 'others did not share many ideas'. According to one member, 'some group members agree with the rest of the group in order to prevent a potentially stupid remark' that could lead to loss of face. This is also an example of the 'Abilene paradox' that was mentioned earlier. Another group member connected loss of face to the good outcomes of group work. He said: '(Now the participants) have to pay attention, or the group will say: "Our group is doing very bad, very bad". So they need to pay attention during the group work.'

A frequently observed manifestation of fear of losing face is that of replying to a question with a positive answer, even if something is not understood. One of the group members gave an example, describing the behaviour of participants during a provincial training endeavour:

'It is sometimes difficult when they do not know how to do (something) for the first time. We explain all and then ask: "Do you understand?" They say "yes", but when I monitor and ask why they do not do (as I instructed) they say they do not understand... Difficult when lying like this.'

Shyness is another form of uncertainty avoidance that was often observed. On multiple occasions when new approaches were introduced, people started to behave in a very shy and uncertain manner during the introduction of a new and unknown energizer called 'mirror you, mirror me':

The group members first remained seated, then stood up after they were persuaded to do so and started to mirror each other's movements clumsily while giggling uneasily. After a while however, the group members moved around the room, mirroring each other. The older members did that to a limited extent, but the younger members used the whole room (field notes $6^{\text {th }}$ group meeting, $p .1$ ).

Another form of shyness is not being willing to move into the spotlight:

When the researcher asked someone to volunteer to do an energizer, only one group member stepped forward, while the others said they did not know one (field notes $5^{\text {th }}$ group meeting, $p .1$ ). 
Seeking approval is the third form of uncertainty avoidance, of which already many examples have been given. With the exception of one group member, all members sought approval regularly before and after preparing and conducting an activity or session and sometimes even during the activity. Seeking approval was also a problem during interviews. This will be discussed during the reflection on methodology in the next chapter.

The fourth manifestation of uncertainty avoidance is fear of arousing anger. This fear was behind group members failing to inform the researcher that they would be absent from a meeting, or would be unable to complete tasks on time. In all cases, group members let the situation take its course, instead of finding a solution together. A more complex version is the fear of not being polite. Being impolite might anger people. This fear, for example, led to some of the delays in the time schedule of the Training Guideline training, as facilitators were hesitant about interrupting participants who were presenting their group work.

Uncertainty avoidance also had consequences for the way people answered questions during interviews. Response bias was overwhelming. Examples of this behaviour are reflected by the following two quotes: 'I have no idea..., but maybe', or 'Sometimes I do not know, but I use your idea'. Another form of the same issue was compliance with the researcher. For example:

The researcher: 'Do you think the participatory approach is better?'

The interviewee: 'Yes, better!'

Or, in another example:

The researcher: 'So the Training Guideline made it more clear?'

The interviewee: 'Yes, more clear!'

The habit of providing 'desired answers' was also profuse, for example: 'I think your answer I know (I think I know the answer you are looking for)'.

\subsubsection{The fourth cultural dimension: femininity versus masculinity}

Formality and status were identified as the important aspects of this dimension. As has been said previously, the working group never gained a formal status 
within MoEYS. Such a status would have strengthened the position of the working group within the Ministry.

Status was also found to be of importance for the individual profiles of the group members. The impact of having followed a professional development trajectory on their status in their Departments was a serious issue. The younger group members whose status was lower than that of the others wished to 'show the colleagues what was learned', in order to raise their status in the Department. They succeeded, as they were both asked to join Departmental working groups in order to inform the others as to how professional development endeavours could be made more effective.

The same status-enhancing desire was possibly behind the repeated requests for a training certificate by the group members. It was found impossible to provide the group members with such a formal certificate, because the working group lacked an official status. Instead, a 'letter of proof', signed by the researcher and the Education Program Manager of VSO (as the supporting organisation), was sent to the group members. The letter was received politely, but clearly conveyed as a poor replacement of a certificate.

Another status issue applied to two of the more senior group members who perceived themselves as highly educated. They tended to show this by using many technical terms and concepts. In some cases, however, terminology and concepts were used incorrectly. For example, one of them said, 'the participatory approach is very important for the modernization of the globalised, the global world', but was unable to come up with any concrete examples. Such vague and false use of language was often confusing for the other group members and participants. The researcher realized that these misconceptions would need to be corrected before they could take root in others. This was found to be difficult at times, because it involved correcting mistakes made by people the researcher relied on. The relationships the researcher had with these two group members was good, and needed to be maintained. Discussing the issues one-to-one, instead of in front of the group solved the problem. With the group member responsible for the Training Guideline, for example, regular private meetings were held, in which the researcher openly addressed issues like his absence: 
He (the group member responsible for the Training Guideline) immediately started by apologizing for not having finished the translation. We discussed why it was delayed and how we could ensure that the translation would be finished as soon as possible (field notes private meeting, 17 March 2008).

This would have been impossible in front of the big group, because of the possibility of losing face.

Status was also important at a motivational level and was supportive to learning. The aforementioned long opening speech given by the Deputy Director of the DGE at the first day of the Training Guideline training provided status to the training in order to motivate the participants to learn.

\subsubsection{The fifth cultural dimension: long term versus short term orientation}

Critical thinking and planning skills were identified by the researcher as the most important aspects of the long term/short term dimension. However, critical thinking was found to be weak in general. For example, when group members were asked about their opinion on the result of the working group, all group members gave answers like: 'The content is very interesting, because I get new experience'. In general, it did not seem to matter much what the experience was, as long as it would lead to more experience of any kind.

A few members' critical thinking skills, on the other hand, had improved:

One of the group members asked: 'Why do we do like this and not like that?' (field notes $2^{\text {nd }}$ preparatory meeting, p. 4).

And during the Training Guideline training:

She (the facilitating group member) had included the importance of hierarchy in the role-plays (field notes $1^{\text {st }}$ training day, p. 5).

Another example was the group member who decided to take more time for his session, after his session was given less time: 
When the researcher asked him what had made him decide to take an additional 25 minutes for his session, he replied that 'this session is very important because it gives people the skills for the participatory approach (field notes $2^{\text {nd }}$ training day, p. 9).

Although such critical thinking was rare, and perhaps because of its rarity, these instances have great significance for the scenario and the learning capacity of the group members.

Planning was the second important aspect and was already described as problematic, both during the preparatory meetings and during the entire Training Guideline training. The consequences of such time deviations were not anticipated, or not found to be of importance by the group members.

\subsubsection{Overall influence of culture on the scenario}

Power issues were found to reinforce uncertainty avoidance behaviour, and thus were a threat to the safe learning environment in which trust was identified as crucial. Group members were often reluctant to correct mistakes made by other

group members, especially during the Training Guideline training, in the hope that the researcher would correct the mistake. In reality, making mistakes was not seen as an opportunity to learn, although that was often suggested by the group members themselves. The fear of making mistakes and of losing face was abundant, and to a certain extent, masked by shyness. Furthermore, it turned out to be difficult to strengthen the weak academic skills like critical thinking, problem solving and planning. Power dynamics were sometimes found to have a further limiting effect on critical thinking, or at least in the articulation of it, as less senior group members did not always feel free to speak their mind.

The issue of power was also connected to the personal status of people. Group members hoped to raise their status in their Department by their enrolment in the working group. There was also a tendency to delegate activities that were seen as less formal to the younger and less senior group members. This was especially the case for the training games during the Training Guideline training. A positive influence of status on the group members' learning was the status-enhancing influence of the support to the scenario and the Training Guideline training expressed by the Deputy Director of the DGE in the CBCG meetings and at the start of the Training Guideline training. 
The lack of academic and meta-cognitive skills such as critical thinking, planning and problem solving, is a widespread problem in Cambodia, influenced by recent history. The effects of this lack are likely to be reinforced by hierarchy, which does not always allow critical questioning of decisions and approaches. It is, however, at odds with the wish to create a participatory working environment in the Ministry.

\subsection{CONCLUSION}

The scenario was certainly successful in identifying cultural influences on professional learning in Cambodia. A large power distance and high levels of uncertainty raised an increased need for establishing trust and safe learning environments. Position and status both played an important role in the extent to which staff was able to utilize and disseminate acquired knowledge and skills. Long-term orientation was found to be weak. Planning skills, including time management, critical thinking skills and meta-cognitive skills were problematic and found to be too difficult to teach the group members during the scenario. This was partly because of the ad hoc way the work in MoEYS is sometimes organised, but is also due to the fact that many Cambodians were never educated in these academic skills. On the other hand, they learned to cope with this lack of skills by applying a great deal of flexibility.

The enacted scenario was partly consistent, as most of the components of the spider web were tuned to one another. The scenario was well embedded in the context, and was able to deliver products that were academically sound and appropriate for that context. The Training Guideline document and its tools were observed to be used in the daily practice of professional development in MoEYS. Time and location were the two weakest components of the scenario. The location for the working group meetings was not appropriate for the methodologies, but also raised the adaptive skills of the group members in order to find ways the methodologies could still be used. Time was the most problematic. Lack of time to join the meetings was the main reason why the less effective group members could not develop towards the levels of the core group members. Most of the group members indicated that they lacked the time to read the academic background information that was provided to them during the scenario. It is unclear whether they really lacked the time, or whether the 
provided information was too difficult. All information was summarized and presented by the researcher in order to ensure understanding on the one hand, and the creation of sound materials and operationalisations of terminology and concepts on the other.

Professional learning based on socio-constructivist principles was quite effective. Group members operationalised terminology and concepts for professional development, which were used correctly during later PDAs. Utilization of training methodologies was less successful. Only two members of the core group improved to the level of 'artisan' facilitators, as they were not only able to make decisions on the application of methodology, but also to adapt it to other contexts. These two members were characterised by high motivation and by enjoying either the position or the support of their superiors to experiment with the acquired knowledge and skills. Both were young and usually present at the meetings of the working group. Yet, given the constraints of the cultural context, their reaching the artisan level is a significant indicator for the effectiveness of the scenario. For these participants, the confluence of external factors, such as support from their Departments, and internal factors, such as personal motivation, enabled them to derive the best advantages of the learning opportunity.

The other three core group members were able to talk constructively about methodology and the need to adopt participatory and learner-based methodologies. They also had been present at almost all meetings and were enthusiastic, but had lesser opportunities to experiment outside the scenario. Support of superiors was similar to that of the other two 'core' members, but they were more reluctant to practise with the acquired knowledge and skills outside the scenario. In other words, despite having the benefit of most of the external factors, internal factors or the personal characteristics of these participants may have had a strong influence on their learning, reducing the effectiveness of the scenario on their learning.

The other four members of the working group were found to be absent too often to really engage in the learning that took place during the scenario. Their absence was partly linked to their position and status, as they were often called away to other meetings. For these participants, it appears that any internal factors or personal characteristics they may have brought to the scenario were outweighed by the extensive negative external factors, which in turn negatively affected their learning and rendered the scenario least effective for them. 
Although the numbers are too small to make reliable judgements on the importance of characteristics of the group members and their influence on the outcomes, it seems that gender, education and experience did not play major roles. Although the two most successful group members were both young, there were also younger members in the other two categories, thus not allowing generalisable judgements about the influence of age.

The scenario was relevant, as it answered or responded to a genuine need for more effective learning during professional development endeavours, articulated not only by the Ministry, but also by the group members. As presented earlier in this chapter, the group members did not only say that they found what they developed and learned useful, but they were also observed to use it in practice. The scenario was able to base its outcomes on 'state-of-the-art' knowledge about professional development, which was contextualised to fit the Cambodian setting. Knowledge about cultural influences on learning in Cambodia was absent, and was therefore investigated as a part of this research. Both the contextualisation of present knowledge on professional development and the findings on culture and learning in Cambodia contribute to a broader knowledge base for professional development and learning in Cambodia.

The scenario was also practical. The acquired knowledge and skills were observed being utilised. The Training Guideline document and its tools were used, not only during the Training Guideline training, but also outside the scenario. The practicality was a result of the extensive collaborative development process executed by the working group. The continuous 'fine-tuning' and contextualisation of the process and products ensured widely usable and meaningful outcomes.

The effectiveness was high for the products, but less successful for the capacity building of the working group members. Only two of the nine group members advanced to the artisan 'facilitator' role level as described by Malvicini (2006), and showed more 'artisan' behaviour. Three other members were on their way to that level. Although more group members could have reached more advanced levels, the reality in the Ministry lowered those outcomes. Main influences were time constraints and other demands on the group members that limited their presence at the meetings. Nonetheless, compared to where the group members had started, all members acquired better understanding of how to organise and 
facilitate professional development endeavours. They were also able to use the contextualised and academically grounded conceptual framework for professional development, which they themselves said was useful. But individual learning progress could have been higher, if the working group had acquired official status and the group members had been more supported by their Departments.

The next chapter will discuss the findings of this research in detail, and will provide recommendations for improving professional development. 


\section{CHAPTER 7 Discussion}

\subsection{RECAPITULATION OF THE STUDY}

\subsubsection{Research context and question}

The Ministry of Education, Youth and Sports (MoEYS) in Cambodia has committed itself to the Dakar millennium goal for education to provide basic education to all children in the country by 2015. This task is tremendous for a country that is still recovering from a thirty-year civil war and is trying to find its niche in a globalised world, but lacks civil staff with enough professional capacity to support such development. Other war-torn countries, such as European countries at the end of World War II, had similarly devastated infrastructure, but still had human-resource capital. Unfortunately, the effect of professional development activities in Cambodia over the past ten years has been limited. As stated by the Deputy Director of the Directorate General of Education (DGE), supported by the findings of Beynon et al. (2004), large amounts of training have been provided in order to build the capacity of Government staff, but acquisition and application of knowledge and skills in the workplace has remained low. There are many reasons that can be identified for the low levels of application, including the lack of a more integrated HRM system. For the learning component of professional development, however, the low levels of knowledge and skills acquisition and application in the work place are mainly caused by the lack of effective and contextualised learning approaches with emphasis on collaborative learning, opportunities to practise and provision of appropriate support. Development partners agreed with MoEYS that many previous professional development projects to strengthen MoEYS staff capacity were far from effective, and said that the staff itself should be included in any future scenario to be developed. Too many manuals, scenarios and plans had already ended up on 
bookshelves in the past. Therefore, MoEYS wished to strengthen professional development in a more effective way.

Since 2005, MoEYS has acknowledged the need for coordinated capacity building and has installed a Capacity Building Coordination Group (CBCG). This group sought to coordinate the professional development activities (PDAs) that take place in all the Basic Education Departments clustered under the DGE. In 2006 and 2007, the CBCG developed a multi-year capacity building plan for the DGE (Berkvens, 2007a), including the development of a Training Guideline. The Training Guideline project was expected to improve facilitation skills of MoEYS trainers, and provide ideas about how professional development could be made more effective.

This combination of goals formed the base of this study, and is represented in the following main research question:

What characteristics should an effective professional development scenario have, when its aim is to strengthen the training facilitation skills of Ministry of Education staff in Cambodia?

The research question can be divided into three sub-questions:

1. Which principles for the design of such a scenario can be derived from a context analysis and a literature review?

2. What are the effects of such a scenario on the acquisition and utilisation of knowledge, skills and attitudes by the staff members?

3. To what extent is the learning of the staff members influenced by (organisational) culture?

An educational design research approach was adopted to answer these questions. The design consisted of three phases: a preparatory phase (consisting of the context and literature analyses), a design and implementation phase, and an evaluation phase.

\subsubsection{Preparation of the scenario}

The preparatory phase consisted of a context analysis and an exploration of the existing academic knowledge base on human resource management, professional development, curriculum development, adult learning and culturally relevant 
learning. The findings informed the development of a scenario for professional development that aimed at increased relevance, consistency, practicality and effectiveness through better understanding of the context.

The context analysis provided useful input on the influence of general and organisational culture on learning processes in Cambodia and MoEYS, and emphasized the need to approach professional development in a culturally sensitive and contextually relevant way.

Several design principles for the scenario were identified during the context analysis. The most important principles aimed at allowing enough time to build trust, gaining a thorough understanding of power issues and how they could influence the scenario, and ensuring the participation of every Department and senior officials in the Ministry in order to develop a broadly relevant, consistent, practical and effective scenario. Furthermore, it was found that Cambodian culture and its influence on professional development processes needed to be explored more thoroughly in order to gain better understanding.

The context and literature analyses were unable to provide enough culturally relevant information on learning processes in Cambodia. Relevant theories to support adult learning in professional development trajectories are informed by Western-based literature. The main design principles gleaned from the literature review were to form an interdepartmental community of learners that would adopt a collaborative 'learning by designing' approach (Lowyck, 2001), based on notions of the corporate curriculum as advocated by Kessels (1996) and socioconstructivist learning theory ( $\mathrm{Ng} \&$ Bereiter, 1992). Sharing of knowledge, skills and experience in a collaborative setting and a rich environment in which the group members could construe their own knowledge were of key importance. Participatory and activating learning methodologies further supported learning in such a collaborative setting. Furthermore, meta-cognitive and regulative and communicative skills, including reflection, critical thinking, and planning needed to be strengthened. All these theories, approaches and strategies were culturally contextualised in order to fit the Cambodian context. On the periphery, the important senior staff members surrounding the research, were involved in or kept informed of the progress of the scenario, and a more integrated approach towards professional development in HRM strategy was promoted by the researcher whenever possible. 


\subsubsection{Design and implementation of the scenario}

This phase started with the establishment of the initial scenario design, which was informed by the design principles identified during the previous phase. The initial design evolved into the enacted scenario during the collaborative work of the community of learners. The CBCG and the Deputy Director of the DGE approved the initial design of the scenario. Enacted, the scenario consisted of eleven working group meetings, three preparatory meetings for the four-day Training Guideline training and the four-day training itself. During the working group meetings, the group members acquired new knowledge and skills while working with the content. The materials developed and the newly acquired knowledge and skills were continuously improved using a micro-cycle approach consisting of iterative cycles of design, testing and revision, leading to gradually more 'finetuned' outcomes.

The micro-cycle approach led to the establishment of a shared frame of definitions and methodologies, or a conceptual framework, for professional development. Furthermore, the working group produced contextualised and academically grounded evaluation forms and checklists for the different phases to organise training, using the micro-cycle approach. The micro-cycle approach also worked well for the acquisition of training methodologies, but it was more difficult to test the methodologies in 'real-life' settings. This was because the opportunities to test methodologies outside the working group meetings were scarce, and not all

group members felt comfortable enough to use the new activities in the training sessions and workshops they conducted outside the scenario. Therefore, the Training Guideline training offered many opportunities to test and experiment with methodologies.

\subsubsection{Evaluation of the enacted scenario}

The final phase of the research was the evaluation of the scenario. The evaluation took place after the end of the scenario.

A set of three models was used to carry out this evaluation:

1. The curricular spider web (van den Akker, 2003)

2. The critical levels of professional development evaluation (Guskey, 2000)

3. The 5-D model for cultural differences (Hofstede, 1986; Hofstede \& McCrae, 2004; Gervedink Nijhuis, 2008). 
The curricular spider web was used to evaluate the relevance and curricular consistency of the enacted scenario against the intended scenario, using the set of components for curricula presented by the model and based on observations, interviews and informal conversations. The critical levels of professional development evaluation were employed to investigate the practicality and effectiveness of the scenario. The first three levels (how learners perceived the scenario, what they learned from it, and how it was supported by MoEYS) were informed by the interviews and the formative evaluation findings. The last two levels (how the learners used the acquired knowledge and skills, and the effect of the scenario on participants of PDAs organised by group members outside the scenario) were mainly enlightened by the observations of the Training Guideline training sessions. The 5-D model for cultural differences was used to assess the practicality of the scenario, based on observations, interviews and informal conversations.

\subsubsection{Main findings}

Any scenario that aims at making professional development more effective in Cambodia should be based on a set of design principles that are both academically sound and contextualised to the Cambodian historical and cultural setting. For this research, such a set of design principles was established. This set is presented below.

The extensive preparatory phase of almost one year allowed the researcher to develop contextual understanding and to build trust in the Ministry, both with senior officials and office staff. Being able to speak and understand the local language helped in this process. An extended period of time before the actual scenario started also made it possible to carry out a broad exploration of needs and wishes.

A community of learners, consisting of representatives of all Departments involved who worked collaboratively using a 'learning by designing' approach was vital in order to arrive at widely shared outcomes. Outcomes were a contextualised and academically grounded conceptual framework for professional development, a Training Guideline document, checklists for developing professional development endeavours, evaluation forms for evaluating those endeavours, and improved facilitation and PDA organisation 
skills of the learners. The community of learners was a reflection of the variety in age, experience and education levels of MoEYS staff, although the Department Directors had selected the members. The diversity of the community of learners allowed for different points of view on topics.

Creating a safe learning environment and building trust with and between learners are both essential for any learning activity in a country in which trust has been so heavily violated over such extended periods of time, but even more critical when applying a collaborative learning approach. Furthermore, learners need to be taught to share knowledge, information and points of view. The creation of such an environment, however, was complicated. Cultural notions of hierarchy, humility, high levels of uncertainty and fear of losing face severely influence the way Cambodians work together. Adapting learning activities to the cultural context, on the one hand, and providing communicative and collaborative learning skills on the other, help to create a learning environment that is suited for such a 'learning by designing' approach. An important finding is that learners possess multiple strategies to cope with cultural dilemmas. In that sense, the concept of a safe learning environment should be contextualised as well.

Meta-cognitive, academic and regulative skills are important for learners when engaging in collaborative learning with emphasis on taking responsibility for the learning process (one of the notions of the corporate curriculum approach advocated by Kessels (1996). These skills are extremely difficult for Cambodian learners to acquire. Openness to reflection and critical examination towards one's own behaviour is weak, as are planning skills and time management. These metacognitive skills were not taught to the current Ministry officials during critical development years, when the country was at war. Furthermore, the educational system did not, and still does not focus on teaching critical thinking skills. Teacher reliance (Esposito, 2005) remained high throughout the scenario.

The incorporation of the training components model (Joyce \& Showers, 2002) ensured more effective acquisition and application of knowledge and skills. Attendance during the group meetings influenced acquisition levels: The learners showing the least progress were those with the highest absenteeism. These learners did not experience the entire scenario and thus did not experience the successive training components aimed at boosting acquisition and application. Furthermore, they did not experience the micro-cycles that gradually built understanding and improved skills. Absenteeism was caused by lack of support from the learners' 
Department Directors and high workload of the group members. Application levels were influenced by the learners' willingness to practise and opportunities to experiment with the acquired knowledge and skills in and outside the scenario. Willingness to practise was determined by personal uncertainty levels and to what extent people are secure-oriented (Huber \& Roth, 1999). Opportunities to experiment both inside and outside the scenario were influenced by attendance levels, which were a result of personal commitment and the opportunities provided by the Department Directors, and as such, a matter of support.

Support from senior officials is essential in order to arrive at more effective professional development learning. The support of His Excellency the Director General and the Deputy Director of the DGE was crucial for the establishment of a working group. The support levels of the Department Directors determined the attendance and utilization levels of knowledge and skills. The difference in progress between learners who were fully supported by their Directors and those who were not is significant.

The researcher supported the learning processes by adopting the cognitive apprenticeship model (Brandt, Farmer \& Buckmaster, 1993). This model was appropriate for support of learning during the scenario. Peer support, i.e. support provided by the fellow-learners, was less successful.

The prolonged time-span of the scenario allowed for greater contextual understanding of how culture influences learning. In general, it can be concluded that history and culture have a strong influence on learning in Cambodia. Collectivist dispositions are severely weakened, with high levels of distrust as a result. At the same time, collectivism was valued and experienced as important during the collaborative learning that took place during the scenario. Power distance is wide, and hierarchical issues especially influence the extent to which learners feel free to express their opinions. Together with uncertainty avoidance, learners do not always feel free to provide feedback to other learners. High levels of uncertainty avoidance limit the willingness to experiment with new training methodologies and techniques. Masculine notions, especially status, influence the willingness to reflect on oneself and the motivation to learn in both positive and negative ways. In general, Cambodians are short-term oriented, which negatively influences planning and critical thinking skills. Despite these cultural issues, the most successful group members overcame these constraints and tried to experiment with the newly acquired knowledge and skills. 
All group members adopted and used correctly the concepts and terminology of the developed conceptual framework for professional development. At the execution level of facilitation, however, the group members showed more variety. Two of the nine group members made an enormous amount of progress and became strong learning facilitators. Three others also made a lot of progress, but had fewer opportunities to practise: they improved, but to a lesser extent. The four other group members also made progress, but they did not progress beyond the level of mechanical use of activities and methodologies.

\subsection{DISCUSSION OF THE MAIN FINDINGS}

\subsubsection{Evaluation criteria}

The set of criteria against which the outcomes of professional development endeavours in developing countries are evaluated needs critical attention. In the past, results have been measured against Western-based criteria measuring only how it should be, instead of against what learners accomplished in relation to where they had started (Peterson, 1997). Many aid organisations in Cambodia also use these goal-based criteria that are inappropriate to the context (see Beynon et al., 2004). Failing to measure against where learners were and what they accomplished does not do justice to the progress made by the community of learners. Therefore, the progress made by the learners and the factors influencing it, has been measured in this study.

\subsubsection{The necessity of a shared conceptual framework}

The need for a conceptual framework for professional development was necessary because soon after the start of the scenario the researcher found that the different Departments not only used various definitions, concepts and approaches for professional development processes, but also that professional development terms and processes were poorly understood. Even needs analyses conducted by internationally established research organisations were poor at acknowledging the weak conceptual understanding by the Government staff they worked with and upon whose responses they based their recommendations (see Beynon et al., 2004; Godfrey, 2000; Siddiqui, Strickler \& Vinde 2004). The many previous professional development endeavours have probably provided MoEYS staff with 
oversimplified representations of concepts and approaches, and done so in an uncoordinated way. During the scenario, the working group developed a clear, academically sound and practical conceptual framework. However, the serious risk of false clarity remains. False clarity occurs when subjects are represented and interpreted in an oversimplified way, without acknowledging what the content fully encompasses (Fullan, 2001). Cambodian facilitators often use oversimplification in order to explain difficult content to less educated people. This further reinforces false clarity and superficial understanding.

The scenario allowed the community of learners to develop the conceptual framework collaboratively and helped the community of learners to place approaches, concepts and activities in a coherent structure. The way the structure was developed in the scenario improved learners' understanding of these processes and methodologies. It also led to shared, validated and contextually relevant content for the Training Guideline. Such a method for arriving at a common and shared language has been described as the psychological approach of collaborative capacity building (Lowyck, 2001). Furthermore, the scenario was able to develop a system dynamic perspective, as it broadened the understanding of the system of powers and interdependent relationships leading to higher external consistency (Kessels, 1993) and formed a commonly shared working system (Lowyck, 2001), which was finalized in the Training Guideline document.

It was important that the conceptual framework developed during the scenario was academically sound. The provision of (academic) background information contributed to the soundness of the conceptual framework on professional development that was established, including definitions for processes like monitoring, evaluation and follow-up. The researcher provided background information in the form of articles, summaries of publications and schematic overviews. The majority of the group members, however, did not use the texts because of time constraints, as they had explained. Another possible explanation is that the English language skills of the group members were not adequate but to admit that might be seen as a form of failure. This hypothesis is supported by the finding that at least one group member said that his English language skills were not good enough to understand the '100 training games' book (Kroehnert, 1991) that was provided to the group members. This book is written in much simpler English than the articles. The researcher responded to this problem by providing the learners with the academic writings and summaries, and also presenting the content during the working group meetings. 


\subsubsection{The need for a safe learning environment}

The development of a universal document for professional development in MoEYS, i.e. the Training Guideline, would have been problematic if people had not developed the shared conceptual framework. A shared conceptual framework and the Training Guideline document could only be established when the group members were willing to explore, discuss and revise their espoused theories and theories in use (Schön, 1967). Such activities in which people are asked to explore and change their beliefs could only take place in a safe learning environment. The need for a safe learning environment has been mentioned by Tomassini (2001), and is strongly emphasized for the Cambodian context by the research described in this dissertation. The events of Cambodia's recent history have disturbed trust between people, calling for extra attention when establishing learning environments and groups of learners.

\subsubsection{The collaborative 'learning by designing' approach}

The collaborative 'learning by designing' approach (based on notions from the corporate curriculum approach as advocated by Kessels (1996) and socioconstructivist learning theory ( $\mathrm{Ng} \&$ Bereiter, 1991) adopted for the scenario was quite successful, but also unfamiliar to the learners. During the meetings, many different participatory and learner-based learning activities were introduced to the community of learners in order to extend their training methodology repertoire. Many of these activities required more time than lectures and used simple stationery, which is rarely provided by the Ministry. Most capacity building endeavours, however, are conducted with funding from external partners. These partners stimulate the Ministry to modernize their professional development activities, but at the same time they operate obsolete budgetary rules. This leads to contradictory situations in which donors, on the one hand, demand more participation of participants during professional development activities, but refuse to pay for the basic necessities required to do so on the other. Fullan (2001) describes such a state of affairs as the difference between adoption and implementation. He argues that the ambitious ideas of supporting organisations are nearly always politically driven and that these political decisions often aim at fast adoption, but fail to attend to matters of quality implementation. This leads to low quality in terms of poor practicality. 
Aside from the aforementioned constraints, the scenario showed that participatory and learner-based learning activities can be effectively used in Cambodia, but that contextualization is necessary. Many Cambodians are not used to activities that ask for their opinion and active engagement. Hierarchical power issues and high levels of uncertainty avoidance reinforce initial reluctance to engage in such activities. Confidence in one's own abilities, high self-esteem, and support from and trust in others are necessary conditions for collaborative learning (Beard \& Wilson, 2002; Malvicini, 2006). It is precisely these characteristics which are problematic for many Cambodians. Again, time is essential to build trust, raise people's confidence and self-esteem, and to allow people to experience the power and value of working collaboratively.

\subsubsection{The threats of a participatory approach}

The viewpoint that participation can be a threat to the empowerment of people (Cooke \& Kothari, 2001; Malvicini, 2006; Wright, 1997) did not apply to the scenario. However, the participatory approach allowed people to take full control over their own learning. However, the threat that does exist in Cambodia regarding participatory activities is the increased risk of 'Abilene paradoxes' and 'group think', as Cambodian culture already has a strong tendency to prevent loss of face and conflict. The 'Abilene paradox' shows that sometimes people tend to agree with decisions they do not approve of, in order not to be troublesome to the group. With 'group think', members of a group share the same ideas without critically testing them, in order to prevent conflict. Both were frequently observed during the scenario.

In order to prevent such risks of a participatory approach, people must be taught to think critically and to articulate their opinion in order to work constructively together. Moreover, academic and meta-cognitive skills in Cambodia are weak as a consequence of the historic educational situation (Harmer, 1995). The researcher found that it was difficult to teach academic and meta-cognitive skills to the group members. Teacher reliance remained high (Esposito, 2005). This finding is quite problematic. It unveils a strong need in Cambodia to develop academic and metacognitive skills in a context that has a weak base for the acquisition of such skills and therefore depends on others to make comparison assessments and decisions, thereby further strengthening such teacher reliance. Unlearning teacher reliance is necessary for learners to develop academic and meta-cognitive skills, but reliance on teachers can only be decreased if learners acquire these skills. 
On the other hand, learners were found to be able to make collaborative decisions during the micro-cycles when reflecting on previous experiences. The important difference was that, in this case, reflection was on an activity or product, not on the behaviour of learners themselves. In general, critical reflection was difficult for the learners. The group members easily gave positive feedback to the researcher, but also expected only positive feedback in return. Most of the constructive feedback that was provided was experienced as failure instead of an opportunity to improve, and as such is a form of cultural uncertainty avoidance. The scenario was not able to change this situation substantially. Much more time should be allocated for activities that explicitly strengthen academic and meta-cognitive skills. Even then it is not guaranteed that learners will be able to turn these complicated skills into practice, as the historical and cultural background is not supportive towards these skills. The practical theory (Handal, 1991) shows that critical reflection on and thinking about one's actions is very difficult. Handal identified three levels of reflective practice (on actions, on the practical and theoretical reasons for it, and on the ethical justification) and showed that educators in general seldom progress beyond the first level of reflection, i.e. the level of planning their actions. Only a small number of educators are able to justify their choices on practical or theoretical grounds. The third level (ethical justification) is almost never reached.

According to Brookfield (1987), people become critical thinkers when they develop an awareness of the assumptions under which others, and they themselves, think and act in a certain context. He claims that critical thinkers become sensitive to alternative ways in which to act and experience the world; instead of being satisfied with 'quick fix' answers, single solutions to problems and representations of universal truths. Critical thinkers make their own judgements, choices and decisions by taking responsibility, rather than following the ideas of others who would do that in the collective interest of the group. In doing so, they are actively engaged in creating their own personal and social worlds, or as Brookfield puts it, 'they take the reality of democracy seriously' (pp. ix-x). When the notions of critical thinking of Brookfield are applied to the Cambodian context, it becomes clear why critical thinking is so difficult for Cambodians. First of all, the large power distance hampers true democracy. Secondly, the more collectivist orientation does not favour individual responsibility and creating individualist 'worlds' by questioning the way the collective has done things for extensive periods of time. Judging others is difficult 
in such collectivist settings, and this difficulty is probably strengthened by the events of the recent past. Being exceptional was dangerous, and people survived the Khmer Rouge time by being inconspicuous (Fröberg Idling, 2009). People learned not to question anything. The tendency not to question things is also observed in classrooms (personal observations, May 2006). Given the Cambodian context, much more is needed to build capacity for critical thinking and other academic and meta-cognitive skills. For instance, the recently started USAID 'young leadership program' aims at strengthening academic and meta-cognitive skills of a new cohort of young potential Cambodian Government staff.

While the learners were engaged in group work, they found that working collaboratively could sometimes create tension. Such tension was culturally reinforced by power differences and uncertainty avoidance. Fortunately, the group was strong enough to resolve these issues, as far as the cultural setting allowed. As has already been said, power issues influenced learning processes and possibilities of application of the acquired knowledge and skills in the workplace. Furthermore, fear and uncertainty were important cultural factors that the researcher needed to understand before alternative learning activities could be introduced. Cambodians themselves were found to have alternative methods of coping with these complicated cultural issues. Collaborative work was possible, but within the existing cultural system. The sole fact that these cultural issues are difficult for foreigners to understand cannot be a reason to try to change the cultural system.

\subsubsection{Support to learning}

Adopting and trying to implement participatory learning approaches in a context that is not used to these approaches means that support to the changing roles of learners, facilitators and leaders is crucial (van de Poll, 2005; Wright, 1997). Although a more learning supportive HRM system was lacking, it did not mean that there was no support for the scenario. On the contrary, the Director General, the Deputy Director of the DGE and the majority of the Department Directors were supportive to the scenario. Their support, however, must be seen within the Cambodian cultural context. None of the Ministerial managers was actively involved in the meetings of the working group, as cultural power distance is partly maintained by distance between hierarchical layers. Because power distance and uncertainty avoidance in Cambodia would prevent participants 
openly giving their opinions if superior officials were present, the absence of leaders is more desirable than their presence. This is, however, at odds with contemporary Western literature on change processes. Berman, McLaughlin and associates (1979) argue that one of the strong indicators for involvement of leaders is their attendance at workshops. That would be detrimental for the empowerment of people and the implementation of change projects in Cambodia. Thus, a more subtle form of support that respected Cambodian culture and aimed at adjusting the roles of both leaders and staff needed to be developed. The researcher decided to involve the senior leaders at a distance, i.e. inform them of the progress and discuss the outcomes with them. This led to enough support from the leaders, and did not disturb the learning processes in the working group.

The most successful learners in the working group had supportive Directors, who decided that their staff could be part of the working group and made it possible for them to join the meetings. Some Directors stimulated dissemination in their Departments, but they were exceptions. Again, it was found that there was a tendency for cosmetic or symbolic adoption instead of implementation of change (Fullan, 2001): Some Departments were found to accept the Training Guideline document, but did not see the need for ongoing support for the more important learning process behind it.

The scenario provided the learners with a relatively strong learning environment for experimentation in order to strengthen utilisation of the new learning methodologies. The adopted training components model (Joyce \& Showers, 2003), which combines theory, modelling, practice, and follow-up, was found to be fairly effective. The group members who were present at almost all meetings experienced the whole scenario (containing the four components) and were found to be better able to apply the acquired knowledge and skills in practice than the group members that were not often present. Furthermore, some learners had fewer opportunities and support for experimentation outside the scenario. This lack of opportunity to utilize newly acquired knowledge and skills is described as a poor transfer climate (Holton, Bates, Ruona \& Leimbach, 1989). Poor transfer climate in Cambodia is mainly caused by poor learning supportive management. This unsupportive management is caused by the lack of an integrated HRM approach and reinforced by local cultural power barriers. Budhwar and Debrah (2001) describe how such hierarchical management systems empower their staff less to take responsibility for their work. According to Malvicini (2006), this is 
mainly caused by the fear felt by the leaders of these organisations of losing power. As Cambodia is characterised by a large power distance, it is not surprising that a more supportive HRM system has not yet been adopted, although it has been developed (EFC, Indochina Research \& Span Consultants, 2006). Weggeman (2000) calls for organisational changes in which the top of organisations no longer takes decisions without the involvement of the staff, but such a shift is highly unlikely to take place for some time yet in a society that is firmly based on power distance.

Support was not only desired from the group members' managers, but also from the researcher/facilitator. The cognitive apprenticeship model (Brandt, Farmer \& Buckmaster, 1993) was a good model for the provision of support during the scenario, as it followed the components of the training model of Joyce and Showers (2002). The researcher modelled behaviour, and offered coaching, scaffolding and fading, followed by assistance on request. Although the cognitive apprenticeship model was effective, it did not acknowledge the strong local cultural uncertainty avoidance factor. Uncertainty avoidance caused even the most successful group members to ask for constant support, where it was no longer necessary. Such secure-oriented behaviour is based in high uncertainty avoidance (Huber \& Roth, 1999). The need for continuous affirmation blocked some of the learners from advancing further.

\subsubsection{Facilitator typology}

It is difficult to provide a clear description of the staff members that developed into the most effective learners. In line with the findings of Joyce, Bush and McKibbin (1982), enthusiasm of learners was found to be a critical factor for behaviour change and openness to learning. Age was not a determinant on its own, but was linked to the hierarchical position group members held in the Ministry. The higher the position of group members was, the bigger the workload and other responsibilities were, thus limiting the possibilities for them to join meetings. But a higher position also meant that members could allocate their own time more freely. It thus seems that motivation in allocating time was also a factor influencing presence. For the less senior group members it was their superiors who decided whether or not they could join. The hypothesis that the more experienced group members would have higher transfer potentials as Thijssen (1996) suggests, could not be confirmed by this research because the more 
experienced group members were often absent during the meetings. Important observations by the researcher were, though, that the older members enthusiastically joined the activities if they were present, and that their contextual expertise largely contributed to the practicality of the scenario outcomes.

The two most successful group members completed the entire scenario of working group meetings, preparatory meetings and the Training Guideline training, and thus also experienced the micro-cycles that were part of the scenario. They were supported by their superiors, experimented with and tried to apply the acquired knowledge and skills in their work. They also asked for support when necessary. Furthermore, they used a mixture of methodologies while facilitating, and were able to adapt their sessions. This more 'artisan' utilization (Joyce \& Showers, 2002) was not observed as often with the other group members, who showed more 'mechanic' forms of facilitation.

Based on the findings, a typology of facilitators can be established. The typology presented in table 7.1 is an elaboration of notions about facilitators from Poell and van der Krogt (1999) and the complicated system of facilitator levels of Heron (1999). The first column shows the five facilitator levels of this elaborated model. The second column describes the corresponding facilitator behaviour of each of the levels. The third column provides the preconditions and constraints that influenced learning of the group members to advance or remain at the different levels.

Table 7.1 Typology of facilitators

\begin{tabular}{|c|c|c|}
\hline $\begin{array}{l}\text { Facilitator } \\
\text { level }\end{array}$ & Facilitator behaviour & $\begin{array}{l}\text { Preconditions and } \\
\text { constraints }\end{array}$ \\
\hline $\begin{array}{l}\text { Novice } \\
\text { educator }\end{array}$ & $\begin{array}{l}\text { Copies authoritarian teaching style as } \\
\text { experienced in school. Emphasis is on } \\
\text { knowledge, without checking learners' } \\
\text { backgrounds and needs, understanding or } \\
\text { progression. No practice or support offered to } \\
\text { the learners. }\end{array}$ & Not observed. \\
\hline $\begin{array}{l}\text { Experienced } \\
\text { educator }\end{array}$ & $\begin{array}{l}\text { Educates mainly by lecturing, but also provides } \\
\text { some exercises. Tries to apply acquired } \\
\text { knowledge and skills, but at basic levels. Has } \\
\text { thought about learners' backgrounds, } \\
\text { sometimes needs assessment is conducted. } \\
\text { Provides support by asking how learners are } \\
\text { doing and explains again when necessary. }\end{array}$ & $\begin{array}{l}\text { High absenteeism during } \\
\text { working group meetings. } \\
\text { Lack of time or will to } \\
\text { practise during and outside } \\
\text { the scenario. }\end{array}$ \\
\hline
\end{tabular}


Table 7.1 Typology of facilitators (Continued)

\begin{tabular}{|c|c|c|}
\hline $\begin{array}{l}\text { Facilitator } \\
\text { level }\end{array}$ & Facilitator behaviour & $\begin{array}{l}\text { Preconditions and } \\
\text { constraints }\end{array}$ \\
\hline $\begin{array}{l}\text { Mechanic } \\
\text { facilitator }\end{array}$ & $\begin{array}{l}\text { Actively generates learning by providing } \\
\text { learning activities and methodologies, but in a } \\
\text { mechanical and not yet fully coherent way. Not } \\
\text { yet able to adjust the learning activities to the } \\
\text { context and needs of the learners. Provides } \\
\text { support by asking how learners are doing, but } \\
\text { does not yet consciously apply scaffold and } \\
\text { fading. }\end{array}$ & $\begin{array}{l}\text { Joined most meetings of the } \\
\text { working group. Practised } \\
\text { and experimented in } \\
\text { protected settings, but } \\
\text { reluctant to practise or had } \\
\text { limited opportunities to } \\
\text { practise outside the } \\
\text { scenario. }\end{array}$ \\
\hline $\begin{array}{l}\text { Artisan } \\
\text { facilitator }\end{array}$ & $\begin{array}{l}\text { Actively supports learning by providing } \\
\text { appropriately selected learning activities and } \\
\text { methodologies, put in a coherent program } \\
\text { (following the Joyce and Showers ,2002, model). } \\
\text { Adjusts program and activities to the context } \\
\text { and needs of the learners. Is able to adjust the } \\
\text { planned activities along the way. Provides } \\
\text { support by providing scaffolds and fades. }\end{array}$ & $\begin{array}{l}\text { Joined most meetings of the } \\
\text { working group. } \\
\text { Had strong support from } \\
\text { manager/leader to join and } \\
\text { apply acquired knowledge } \\
\text { and skills in daily work. } \\
\text { Practised and experimented } \\
\text { in and outside the scenario. } \\
\text { Created time and was } \\
\text { willing to practise and } \\
\text { experiment. } \\
\text { Actively requested support } \\
\text { from the researcher. }\end{array}$ \\
\hline $\begin{array}{l}\text { Catalyst of } \\
\text { learning }\end{array}$ & $\begin{array}{l}\text { Provides the freedom and fully empowers the } \\
\text { learners to learn. Provides support on request. } \\
\text { Optimizes learning conditions by applying } \\
\text { activities and methodologies when appropriate } \\
\text { and requested by the learners. }\end{array}$ & Not observed. \\
\hline
\end{tabular}

\subsection{REFLECTION ON THE RESEARCH METHODOLOGY}

This research was carried out as educational design research, in order to shape an innovative intervention and to contribute to theoretical underpinnings of an intervention (cf. Kelly, 2006) for the complex and poorly understood context of professional development in Cambodia. In this context, the approach is exploratory and developmental, bringing its own methodological dilemmas with it. This section will reflect on the main issues and biases that were experienced and how these issues were resolved. 


\subsubsection{The preparatory phase}

The preparatory phase consisted of a context analysis, a review of the available knowledge base, and an exploration of the needs and wishes of people in the Ministry and supporting organisations. During this phase, the researcher mainly took on the role of researcher.

The biggest challenge was the extremely small knowledge base and the limited amount of local documents that were objective. In both cases, documents were gathered from a wide variety of organisations and private bookshelves, but even then the assembled base of documents was small.

Another problem involved the needs analyses that were produced by international aid organisations in Cambodia. It was found that these were all biased towards the aid organisations' wishes. The intrinsic goal of every needs analysis investigated by the researcher was that the respective organisation wanted to be involved in meeting the needs described in the document. This, of course, did not help the validity of the findings. Furthermore, the needs and wishes of these organisations were found to be different from those held by the Ministry. Also, the aid organisations had strong opinions on how to proceed, informed by documents and needs analyses that were often found to be in contradiction with each other. For the scenario described in this dissertation it was the Deputy Director of the DGE who decided on the direction in the end.

Many of the documents that described Cambodian culture or habits were personal reflections, instead of academically grounded theory. The literature describing adult learning and professional development theory was strongly Western-oriented. None of the academic sources provided knowledge of professional development in the Cambodian context. A small survey to compare Cambodian culture with the 5-D model of cultural differences model (Hofstede, 1986; Hofstede \& McCrae, 2004; Gervedink Nijhuis, 2008) was conducted as part of this research, in order to cover the knowledge gap describing culture and learning in Cambodia.

Educational design research was chosen as an appropriate approach for the research, because of the poorly described context, the small existing knowledge base and the fact that previous interventions had not been able to deliver practical and effective approaches for professional development. Educational design 
research allowed for thorough exploration of the context and acquisition of knowledge about how learning in the specific Cambodian context could be improved. The findings of this research contribute to a broader knowledge base and a better understanding of how learning during PDAs could be strengthened in Cambodia and how Cambodian culture influences learning.

\subsubsection{The design and implementation phase}

The triple role of the researcher/designer/facilitator was most obvious during this second phase of design and implementation. Having so many roles was sometimes difficult. First of all, observing the working group meetings while facilitating them was not always easy. It is inevitable that some events were missed. This was prevented as much as possible by thorough preparation in order to have more time available for observation and reflection on the findings. A clearer separation of the different roles during the successive phases would have been helpful in order to limit further role bias. Furthermore, the researcher worked out the observations immediately after the meetings and often verified observations and interpretations with the group members. An advantage of the different roles, however, was that the researcher was able to adjust activities and approaches on the spot when it was found necessary to adapt the program. For example, when the researcher found that most group members were unable to read the (academic) background information, he inserted a session on the main content of the information, before the group developed academically sound and contextualised evaluation forms. For further meetings, the researcher decided that all (academic) information would not only be distributed on paper, but also summarized and presented.

A second role bias occasionally emerged when group members demanded that the facilitator shared his point of view during differences in opinion between group members. Maintaining a safe learning environment in a cultural setting that is based on prevention of loss of face is complicated. When group members asked the facilitator/researcher to solve the argument between the people involved, the researcher was then placed in a dilemma. The dilemma became further complicated when a more superior group member articulated an academically unsound idea in contradiction to the opinion of a less senior colleague. Such situations were the exception, but certainly not imagined. On occasions where academically unsound issues were involved, the researcher decided to correct mistakes in the least confrontational way, although painful rectifications could not 
always be prevented. Fortunately, this did not negatively affect the relationships between the researcher and the group members.

Another issue that the researcher regularly experienced was a lack of open communication with the group member who had been delegated responsibility for the project by the Deputy Director of the DGE. The researcher often did not know about his absence in advance and other organisational problems were often not discussed. The involvement of this group member was, however, crucial for the completion of the scenario. According to Malvicini (2006), open communication and transparency are important for effective collaboration, but both were weak with this one group member during the scenario.

The two issues previously described sometimes led to insider/outsider bias. Not being kept informed could still be attributed to thoughtlessness, although it meant that the researcher was forced into the outsider role. More intricate was the influence of the foreign origin of the researcher. The fact that the facilitator/researcher was a foreigner gave rise to the expectation that the researcher would solve issues that could otherwise lead to confrontations between group members. For example, the researcher was often informed of a mistake that another group member made, with the expectation that the researcher would address the issue with the colleague. If the researcher decided to act, it was always done during a one-to-one conversation. The second form of insider/outsider bias was language. The researcher had the advantage of understanding and speaking Khmer, preventing bias that could have arisen by using translators, and if translators were used, the researcher was able to judge the accuracy of the translation. This added to validity and the richness of the collected data.

Although the researcher was not able to select the working group members, the group members selected were still a reflection of facilitators in MoEYS. This could easily have been otherwise. For future endeavours, selection of the community of learners should be discussed with the relevant senior staff in advance.

The final issue to be discussed here was the high absenteeism of some of the group members. Their absenteeism had consequences for supporting their learning. They not only missed important parts of the scenario, but also of the micro-cycles. This led to difficulties with evaluating their learning, which will be discussed in the next section. 


\subsubsection{The evaluation phase}

The evaluation of the intervention was based on observations during the entire enacted scenario (working group meetings, preparatory meetings and the fourday Training Guideline training), filled out evaluation forms, final interviews with the group members and records of informal conversations.

During the evaluation phase, the researcher had mainly a researching role, although he facilitated one session during the Training Guideline training. But data were also collected during the previous phase, where the researcher had a triple role. This triple role, as has already been discussed, might have led to some loss of detail in field notes. The measures taken to prevent this have also already been discussed. Another complicating factor was the loss of opportunities to observe the progress of the working group members that were often absent. The researcher tried to observe their progress in training activities they conducted outside the scenario, but that was not possible in all cases. Furthermore, two core group members were absent during the Training Guideline training, leading to the same problem. For these two members, progress has only been assessed using the outcomes of their evaluation forms and final interviews.

The researcher was not able to observe the last day of the Training Guideline training. One of the group members was asked to take notes and photographs of these sessions. The observations were found to be informative but only marginally useful, as too much interpretation was necessary to provide meaning to the notes.

Significant difficulties experienced during interviews were the fear of losing face by the interviewees, the frequent tendency to lower the expectations of the researcher before answering a question, and the provision of desired answers. These response biases were abundant, and all group members showed this behaviour. During the introduction of each interview, the researcher always mentioned the necessity of answering honestly and not to provide desired answers. But only towards the end of the research, when trust was established, interviewees tended to provide more direct answers. When necessary, the researcher returned to questions at a later stage during the interviews, or followup interviews, and asked the question in a different way in order to check for these biases. In addition, findings were triangulated as much as possible. This double-checking and triangulation of data was a continuous point of attention, and was sufficient in order to prevent response bias. 
The provision of desired answers has negative consequences for the reliability of evaluation forms in the Cambodian context. The reliability of these forms is further compromised by the tendency of respondents to positively evaluate all PDAs that deliver new knowledge and skills. As such, evaluation forms lack the power to distinguish between effective and less effective PDAs. The nature of the instrument denies researchers the possibility of verifying answers and identifying nuances, thus limiting the usability of forms as an instrument for evaluation in Cambodia.

\subsection{REFLECTION ON THE SCENARIO}

The quality of the scenario was assessed for relevance, consistency, practicality and effectiveness (Nieveen, 2009) by using the curricular spider web (van den Akker, 2003), the critical levels of professional development evaluation (Guskey, 2000) and the 5-D model for cultural differences (Hofstede, 1986; Hofstede \& McCrae, 2004; Gervedink Nijhuis, 2008).

These three models were used to assess whether the quality of the scenario was appropriate. Sometimes the components of the different models overlapped, which added to the validity through conceptual triangulation. Behaviour described by the five dimensions for cultural differences were also influencing issues described by the components of the other models. But the dimensions for cultural differences allowed for more detailed exploration of the influence of culture on learning, and thus delivered important understanding that would have been lost if the model had not been used in addition to the other two.

The scenario seemed quite consistent, as many components of the spider web were coherent with one another, but two aspects, time and location, were weak in comparison with the others. These two aspects were also the most difficult to change during the scenario, as they are inherent to the context. The same issue applies to practicality, as the model of critical levels of professional development evaluation does not provide a qualifying structure. Therefore, the researcher provided a 'thick' - or rich -description on which the conclusions of the research are based. For future research, the development of a set of definite, but contextualised, measures would be helpful to qualify the outcomes of this kind of research. 
In generalized terms, though, it can be concluded that the scenario was relevant, as it met the needs and wishes of MoEYS and the learners. It was also grounded in academic theory. Given the context, the scenario also seemed consistent. Although the location and time available were not optimal, these aspects were part of the context and difficult to change. The scenario seemed also quite practical in the sense that learners adopted the developed guideline, concepts and terminology. The effectiveness was high when looking at the products that were developed (the guideline, tools and conceptual framework), but varied when looking at the facilitative performance of the learners. Although in terms of numbers the effectiveness is not high, the outcome is still remarkable when it is recalled that group members did not receive any incentives and joining was based on the intrinsic motivation to learn. When the working group would have obtained formal status, the outcomes would have been higher. Also important is that the research was able to identify conditions that influence acquisition and application of knowledge and skills.

If a research project as described in this dissertation were to be conducted again, it would be wise to establish a formal status for the working group. An official letter, signed and stamped by a high-ranking officer would improve the attendance levels of the learners and lead to more support by the learners' superiors, offering more opportunities for application of knowledge and skills by the learners. Learners would then experience the entire scenario with its steps from acquisition to application, on the one hand, and the iterative progression of knowledge and skills developed by the micro-cycles on the other. Qualifying the relevance, consistency, practicality and effectiveness of the scenario would have been easier if definite measures existed to do so. Furthermore, a clearer distinction between the different roles that the researcher had would be helpful to further prevent role bias. An independent observer might, on the one hand, raise reliability of data gathering, but limit the freedom of expression and action of the learners on the other.

\subsection{CONCLUSIONS AND RECOMMENDATIONS}

\subsubsection{Conclusions}

This research was conducted in order to a) identify ways in which learning during PDAs could be made more effective in the Cambodian Ministry of Education, 
Youth and Sports and b) contribute to the academic knowledge base on professional development in Cambodia. The following conclusions can be drawn from this research:

The study has shown that a collaborative 'learning by designing' approach (based on notions of the corporate curriculum approach as advocated by Kessels (1996) and socio-constructivist learning theory ( $\mathrm{Ng} \&$ Bereiter, 1991), can be successful for the strengthening of professional development of Ministry staff in Cambodia. The scenario was relevant, as it was based on a strongly articulated need from the Ministry of Education and its responsible staff. Furthermore, the scenario process and its products were based on and met the criteria of the available academic knowledge base on professional development. Where the base was not solid enough, additional research was conducted in order to close the existing gap. The iterative 'learning by designing' micro-cycles approach contributed to the consistency and practicality of the scenario. The developed products - the Training Guideline document, and its tools - were practical and used by the learners, but the acquisition and application of organisation and facilitation skills by the learners varied.

Although not all the members of the established community of learners for this research developed into artisan facilitators, they all showed progress in their learning and adopted the developed shared conceptual framework for professional development. Two of the group members were found to have reached the level of artisan facilitator, meaning that they were able to adapt activities and methodologies to the needs of their participants. Three other group members advanced towards the novice facilitator level, meaning that they improved, but still performed at more mechanic levels of facilitation. Four members showed some improvement, but not enough to conclude that they progressed beyond the experienced educator level.

Although the cultural and historical backgrounds of the Cambodian context might suggest otherwise, collaborative learning is, indeed, possible in Cambodia. It is important, however, to accept cultural specificities and adapt activities and methodologies to the cultural context. The socio-constructivist approach was key for the identification of such adaptation needs, and thus contextualisation of methodologies. 
In order to be able to work collaboratively, trust must be created first. Trust can only be established over a prolonged period of time and also demands an open and respectful attitude of the facilitator.

The need for strengthening academic and meta-cognitive skills is also apparent. At present, these skills are weak in Cambodia. The lack of strong critical thinking skills combined with high uncertainty avoidance and power issues leads to difficulties with expressing well thought-through opinions. Although this only occasionally led to problems during the scenario, it will have larger consequences in groups of learners that have poorer educational backgrounds.

The training components model (Joyce \& Showers, 2002) and the cognitive apprenticeship model for support (Brandt, Farmer \& Buckmaster, 1993) are appropriate models for the strengthening of acquisition and utilisation of knowledge and skills. The most successful learners were the most actively involved and motivated group members. But for application at the workplace, transfer climate (Holton, Bates, Ruona \& Leimbach, 1989) is also important. However, transfer climate was found to be limited, as the support by Department Directors was not always sufficient to enable the group members to implement or disseminate their acquired knowledge and skills. Stronger support by and involvement of managers and leaders could have strengthened the outcomes of the scenario.

The aim of the scenario was to come to a more effective method for the professional development of staff, not to change the cultural system in which that capacity building took place. Any scenario that would try to change such a cultural system would not be effective, as the cultural system would be found to be far more rigid and solid than any scenario. Project documents would most likely end up on the pile of other 'nicely tried' projects, and the skills would slowly fade from the learners' repertoires. The participatory dialogues in a rich environment that support the learning progress of the group members safeguarded the contextual relevance and practicality of the scenario, also in a cultural sense.

The way this research was conducted delivered profound contextual understanding of the national culture, the institutions and the broader environment (Budhhwar \& Debrah, 2001). All three domains were found to have tremendous influence on professional development in general, but also on group and individual learning processes. Such understanding is crucial for any change projects anywhere. Aid organisations should acknowledge this and stop 
introducing approaches that are not appropriate to poorly understood contexts. This scenario shows that it is much more beneficial to acknowledge local expertise and to gain understanding of local contexts.

It is hoped that the research presented in this dissertation will provide other researchers with ideas of how to apply socio-constructivist and 'learning by designing' approaches in their research settings. Some of the approaches and activities described in this dissertation have also been applied in the Cambodian Ministry of Health (MoH), and were found to deliver similar results. The short description of the South-African situation as described by Groener (2006) provides some ideas for application in that setting as well.

\subsubsection{Recommendations}

For policy making in Cambodia

With the establishment of the Training Guideline, the Cambodian Ministry of Education, Youth and Sports has a viable document in hand to raise the effectiveness of training and workshops delivered by the Ministry. The guideline, however, is just a document to support a far more intricate and important process that started with the Training Guideline working group, but needs to be implemented further if the Ministry is serious in its attempt to professionalize learning in its organisation. The ideas behind the guidelines should not only be adopted for professional development endeavours delivered by MoEYS staff, but also for those that are provided by external organisations. Adopting a more effective approach for professional development also includes a different approach towards leadership and adaptations of the HRM approach. Based on this study, the following recommendations are put forward:

- The study showed that a socio-constructivist approach, with proper support, leads to more effective learning by MoEYS staff. Most professional development provided to and by MoEYS, however, adopts a top-down approach, in which knowledge, skills and experience of the staff is not acknowledged. Foreign facilitators especially have the tendency to 'start from scratch' instead of 'where learners are'. Therefore, MoEYS should recognize the previously acquired competencies its staff members possess and ensure that professional development endeavours take their knowledge and skills into account and include the learners in learning processes. The participatory activities that were used and adapted to the Cambodian context could be used to involve learners in problem-solving, decision-making and policy-making processes. 
- The second recommendation is to acknowledge the facilitator status of the Training Guideline working group members. They have enough experience to support the development of more effective professional development endeavours, inside their own, but also in other Departments of MoEYS. The working group members and their trainees should be enabled and supported to further implement their knowledge and skills in the workplace.

- Furthermore, MoEYS should reserve an appropriate time span and budget for professional development activities that use participatory and learner-based methodologies. Appropriate stationery is also important. When requests for funding are prepared, the budget should be transparent and clearly state what is needed and why. Facilitators should have a clear idea of what activities they wish to use during a training session or workshop, in order to make sure that the appropriate time and materials are available.

- A more integrated HRM approach would be helpful, in which MoEYS adopts a 'learning organisation' approach.

- The academic and meta-cognitive skills of many MoEYS' staff should be strengthened, as planning, critical thinking and reflective skills are found to be low.

- The Ministry should provide formal status to the kind of capacity building endeavours presented in this dissertation. Endeavours, like the one described in this dissertation, are usually executed over a prolonged period of time and the presence of all group members is essential to arrive at collaboratively agreed outcomes. A formal status would make it easier for group members to join the meetings.

For organisations supporting professional development

For donors and non-governmental organisations (NGOs) that provide or support professional development, the following recommendations apply:

- First of all, aid organisations should acknowledge that not all needs and wishes can be decided in advance. In line with Fullan (2001), this research shows that priority needs are often not yet completely clear at the beginning, but become more apparent during the evolution of scenarios. Development projects usually do not allow these important 'advancing insights' that are crucial for effective capacity building, as projects are developed too rigidly.

- Organisations should acknowledge the importance of thorough contextual understanding. Too often, needs analyses and recommendations are based on poor understanding. This leads to interventions that already have their own failure incorporated. 
- Aid organisations should also acknowledge that organisations like MoEYS and its staff have contextual knowledge and skills, which are highly valuable for arriving at practical and relevant outcomes. Too often, the learners are approached as novices. New, or sometimes even the same, concepts are taught, but often in a simplified form. Such uncoordinated endeavours offering simplified content, add to false clarity and superficial understanding by learners, instead of using and strengthening the existing foundations. If new approaches or concepts are introduced, then these need to be placed in the already existing framework of other concepts and approaches.

- Sufficient time and money for effective professional development, and the appointment of strong facilitators are both crucial preconditions for effective professional development. This scenario shows that learning that is established through participation and in dialogue, is more effective than the top-down approach that is often adopted. Strong facilitators are necessary, but they should be allowed enough time to develop contextual understanding. Furthermore, stationery and other learning materials should be available. If aid organisations really want professional development to become more effective, the budget for these activities should be appropriate to allow enough time for learning and sufficient budgets for the purchase of materials.

- Alternative learning in the form of 'on the job' learning does not seem appropriate in contexts that are characterised by poor time management and weak supportive systems. Many aid organisations try to promote such learning because of the lower costs.

\section{For professionals supporting professional development}

For professionals who are involved in professional development activities in Cambodia, the following recommendations are applicable:

- Professionals should develop thorough contextual understanding before introducing any approaches or concepts. This research shows that the Cambodian context and culture are complex, and in the past facilitators of learning too often introduced concepts with the best intentions, but at the same time in a thoughtless and uncoordinated way. This has led to a combination of half-introduced, poorly implemented and superficially understood concepts.

- Experts should modify their actions from a dominant perspective, towards a respectful perspective (Fasheh, 2004) that aims at understanding the context and coming collaboratively to adaptations of concepts, approaches and activities in order to fit the context, thus leading towards more effective 
systems and learning. The scenario described in this dissertation shows that such a respectful approach works.

- Participatory and learner-based methodologies work well in Cambodia, if activities are adapted to the cultural context. Uncertainty, fear and hierarchy are important issues to be aware of when choosing a collaborative approach. Creating a safe learning environment is crucial. Furthermore, people are not used to speaking their minds, but as this research shows, can be empowered and motivated to do so within the existing cultural context.

- When adopting a different learning approach, a strong support system must be installed. The support offered using the cognitive apprenticeship model was found useful during this research. It allows the provision of scaffolding when needed, but also to fade when appropriate. Any endeavour should have a focus on empowering staff to take responsibility for the matter at hand. Fading is thus the ultimate indicator for effective capacity building.

- Respecting hierarchical relationships is crucial, and can be used to raise the profile of the endeavour. First of all, the responsible officials should remain informed at all times in order to prevent rejection of the project later. Secondly, the representation of officials at the start of an endeavour is an important indicator for relevance to the learners. Finally, ongoing involvement of the responsible officials will contribute to the effectiveness of the outcome, as it is checked regularly against the initial mandate.

- Establishing support from senior leaders in order to allow staff to join, the provision of opportunities to apply acquired knowledge and skills and the offer of career opportunities would strengthen acquisition.

- Personal traits like willingness and ability to experiment with the acquired knowledge and skills, and allocating time to prepare and practise further, all strengthen acquisition.

\section{For professional development in a broader context}

Many of the findings presented in this dissertation, and the recommendations given here, might also apply to the broader context of professional development in developing countries, and possibly in developed countries as well:

- Thorough understanding of any context should be developed before the onset of an intervention in any organisation. Every context has its specific characteristics and cultural issues. Too often the context is seen as the problem instead of the base in which an intervention should be anchored. 
- Developing professional capacity is a process involving humans. The people forming an organisation should be taken as the starting point, the intended result as the point to work towards. After those two have been established, the trajectory should be developed. The length of the trajectory should depend on the effort that is needed to bring the two points together. In development work, too often only the end goal and a set amount of time as determined by budgetary constraints are established. Time to build trust should be included in the length of learning trajectories.

- Although education levels might be low, the members of an organisation are not tabulae rasae, but people with contextual experience. That experience should be acknowledged. If people have received education or training before, they probably have learned other concepts, methodologies and definitions. These should be explored and checked for false clarity and misconceptions.

- Many countries are characterized by similar conditions to those in Cambodia. The approach described in this research might help to improve professional development for these contexts.

For the knowledge base on educational design research

For researchers involved in educational design research, the following recommendations apply:

- When conducting educational design research in development contexts, enough time should be allowed to gain thorough understanding of the local context, i.e. culture, organisational culture, the broader environment, but also history.

- Being able to at use at least simple daily-life courtesies in the local language would help to build trust and cooperation. Thorough understanding of, and ability to speak the local language further strengthens understanding and adequate data gathering.

- Being extremely clear about what educational design research is and how it will be conducted is necessary, as many people will not have had previous experience with this form of research. The concept behind 'learning-bydesigning' will also be unfamiliar, but people will soon become accustomed to it.

- Acknowledging and being aware of possible differences between 'espoused theories' and 'theories-in-action' is necessary when conducting this kind of research in countries like Cambodia.

- A system for qualifying the outcomes of developed scenarios would be useful to allow firmer judgements about the quality of scenarios developed by educational design research. 
- A clearer description of and separation between the various roles of researcher / designer / facilitator during the different phases of research would help to create appropriate measures against role bias.

\section{Further research}

This research showed the impact of a scenario for more effective professional development on a small group of MoEYS staff from different Departments in the Ministry. The impact on the secondary beneficiaries, i.e. the participants of the Training Guideline training, was not evaluated in detail. Future research might investigate the long-term effect on the organisational and facilitation skills of the working group members, but also the effects on the work of the secondary beneficiaries.

Other interesting lines of research could explore in more detail how uncertainty avoidance influences individual and group learning, and what possible interventions could strengthen people in becoming more confident learners. It would also be interesting to find ways to strengthen the academic and metacognitive skills of Cambodian learners.

Finally, further research could investigate to what extent the scenario described in this dissertation is also applicable to other post-conflict areas and developing countries. 


\section{REFERENCES}

ADB, (1996). Cambodia education sector strategy study. Manila: ADB.

ADB, (2008). ADB and Cambodia factsheet 2008. www.adb.org/Documents/Fact_Sheets/CAM.pdf

Altner, D. (1999). Building national capacity in education sector management: A UNESCO/UNDP project in Cambodia 1995-1998. In D. Sloper (Ed.), Higher education in Cambodia. The social and educational context for reconstruction (pp. 173-201). Bangkok: UNESCO Principal Regional Office for Asia and the Pacific.

Amstutz, D. (1994). Staff development: Addressing issues of race and gender. In E. Hayes, \& S. A. J. Colin (Eds.), Confronting racism and sexism. New Directions for Adult and Continuing Education Series No. 61, 39-52. San Francisco: Jossey-Bass.

Amstutz, D. (1999). Adult learning: Moving toward more inclusive theories and practices. New Directions for Adult and Continuing Education, 82, 19-32.

Argyris, C., \& Schön, D. (1974). Theory in practice: Increasing professional effectiveness. San Francisco, CA: Jossey-Bass.

Ashton, D. N. (2004). The political economy of workplace learning. In H. Rainbird, A. Fuller, \& A. Munro (Eds.), Workplace learning in context (pp. 21-37). London: Routledge.

Ayres, D. M. (2000). Anatomy of a crisis. Education, development, and the state in Cambodia, 19531998. Hawaii: University of Hawaii Press.

Beard, C., \& Wilson, J. P. (2002). The power of experiential learning: A handbook for trainers and educators. London: Kogan Page.

Belton, V., Thornbury Gould H., \& Scott J. L. (2006). Developing the reflective practitioner designing an undergraduate class. Interfaces, 36(2), 150-164.

Berg, E. J. (1993). Rethinking technical cooperation: Reforms for capacity building in Africa. New York: UNCP/Development Alternatives.

Berkvens, J. B. Y. (2007a). DGE capacity building mid-term plan 2008-2010. Phnom Penh: MoEYS.

Berkvens, J. B. Y. (2007b). Guidelines for effective training and workshop. Phnom Penh: MoEYS.

Berman, P., McLaughlin, M., Pincus, J., Weiler, D., \& Williams, R. (1979). An exploratory study of school district adaptations. Santa Monica, CA: Rand Corporation. 
Beynon, J., Ly, M., Ory, T., Poyck, G., Put, S., Sam, S., \& Yinsieng, S. (2004). Planning and management skill development in the education sector in Cambodia: Report on needs assessment study. Paris: IIEP/Unesco.

Bikales, W. G. (1997). Capacity building in a transition country: Lessons from Mongolia. In M. S. Grindle (Ed.), Getting good government. Capacity building in the public sectors of developing countries (pp. 435-4664). Boston, MA: Harvard Institute for International Development, Harvard University.

Bolhuis, S. (2000). Naar zelfstandig werken. Wat doen en denken docenten? Thesis. Leuven/Apeldoorn: Garant.

Bolhuis, S., \& Simons, R. J. (2001). Naar een breder begrip van leren. In J. W. M. Kessels, \& R. F. Poell (Eds.), Human resource development. Organiseren van het leren (pp. 37-52), Alphen aan den Rijn: Samsom.

Boshier, R. (1991). Psychometric properties of the alternative form of the education participation scale. Adult Education Quarterly, 41(3), 150-167.

Boulton-Lewis, G. M., Wills. L., \& Mutch, S. (1996). Teachers as adult learners: Their knowledge of their own learning and implications for teachers. Higher Education, 32, 89106.

Brandt, B. L., Farmer, J. A., \& Buckmaster, A. (1993). Cognitive apprenticeship approach to helping adults learn. New Directions for Adult and Continuing Education, 59, 69-78.

Brocket, R. G., \& Hiemstra, R. (1991). Self-direction in adult learning: Perspectives on theory, research, and practice. New York: Routledge.

Brookfield, S. (1987). Developing critical thinkers: Challenging adults to explore alternative ways of thinking and acting. New York: Teachers College Press.

Brookfield, S. D. (1991). Understanding and facilitating adult learning. Buckingham, UK: Open University Press.

Brookfield, S. (1993). Self-directed learning, political clarity, and the critical practice of adult education. Adult Education Quarterly, 43(4), 227-242.

Buchanan, J. T., \& Scott, J. L. (1998). Student centred learning in a large, first year management class: History, reflections and future directions. In Proc. Vice Chancellor's colloquium, VITAL, innovations in teaching. University of Waikato, Hamilton, New Zealand, 177-200.

Budhwar, P. S., \& Debrah, Y. A. (Eds.). (2001). Human resource management in developing countries. London: Routledge.

Cambodia's higher education dreams confront reality. (2008, October 5). Agence France Presse. http://afp.google.com/article/ALeqM5httnNxhB029mwA0vdmzEDUnjgDBA

Cambodia seeks foreign aid despite global woes. (2008, December 4). International Herald Tribune. http://www.iht.com/articles/ap/2008/12/04/business/AS-Cambodia-ForeignAid.php

CDRI, (2004). A management framework for capacity development: mandating effectiveness and value for money. Phnom Penh: CDRI. 
Chandler, D. (2007). A history of Cambodia. Boulder, CO: Westview Press.

Chea, V. (2005). VSO placement outline Capacity Building Coordination Officer at MoEYS, DGE. Phnom Penh: VSO.

Cohen, J. M., \& Wheeler, J. R. (1997). Training and retention in African public sectors: Capacity building lessons from Kenya. In M. S. Grindle (Ed.), Getting good government. Capacity building in the public sectors of developing countries (pp. 125-156). Boston, MA: Harvard Institute for International Development, Harvard University.

Collins, A., Brown, J. S., \& Newman, S. E. (1989). Cognitive apprenticeship: Teaching the crafts or reading, writing and mathematics. In: L. B. Resnick (Ed.), Knowing, learning and instruction - Essays in honor of Robert Glaser (pp. 453-494). Hillsdale, New Jersey: Erlbaum.

Contreras, M. E. (1997). Capacity building in the Bolivian social policy analysis unit: Reflections of a practitioner. In M. S. Grindle (Ed.), Getting good government. Capacity building in the public sectors of developing countries (pp. 199-228). Boston, MA: Harvard Institute for International Development, Harvard University.

Cooke, B., \& Kothari, U. (Eds.). (2001). Participation. The new tyranny? London/New York: Zed Books.

Cooke, B. (2001). The social psychological limits of participation? In B. Cooke, \& U. Kothari (Eds.), Participation. The new tyranny? (pp. 102-121). London/New York: Zed Books.

Coombs, P. H. (1985). The world crisis in education: A view from the eighties. New York: Oxford University Press.

Cowan, J. 1998. On becoming an innovative university teacher. Buckingham: Open University Press.

Dewey, J. (1938). Experience and education. New York: Collier Books.

Dirkx, J. M. (1999). Invited reaction: Managers as facilitators of learning in learning organizations. Human Resource Development Quarterly, 10(2), 127-134.

Domrei, (2006). Training needs assessment, final report. Phnom Penh: Domrei research and consulting.

Drucker, P. F. (2000). De opkomst van de nieuwe organisatie. In Harvard business review, over kennismanagement. Zaltbommel: Uitgeverij Thema.

Dyer, C. (2001). Nomads and education for all: Education for development or domestication? Comparative Education, 37(3), 315-327.

EFC, Indochina Research, \& Span Consultants. (2006). Staff development, accreditation and reward systems. Phnom Penh: EFC/Indochina Research.

Ellinger, A. D. (2004). Contextual factors shaping informal workplace learning and its facilitation: The case of 'reinvesting itself company.' In R. M. Cervero, B. C. Courtenay, M. T. Hixon, \& J. S. Valente (Eds.), The Cyril O. Houle scholars in adult and continuing education program global research perspectives, 4, 76-92.

Eraut, M., Alderton, J., Cole, G., \& Senker, P. (1998). Development and skills in employment. Research Report no 5. Brighton: University of Sussex Institute of Education. 
Esposito, E. (2005). Emotional intelligence and andragogy: The adult learner. Paper presented at the $1^{\text {th }}$ International Conference: Learning organisation in a learning world, April $18^{\text {th }}$ 22nd 2005. King Mongkut's University of Technology, Thonburi Thailand.

Fasheh, M. (2004). Rethinking our perceptions, conceptions and values concerning learning, education, and development, as they pertain to adult education, lifelong learning and educational systems. Lecture presented at the Salzburg Seminar, Global Issues: The Roles and Responsibilities of Adult Education, Salzburg, Austria. In Rossiter, M. (2005). Radical Mutuality and Self-Other Relationship in Adult Education. In S. B. Merriam, B. C. Courtenay, \& R. M. Cervero, (2005), Global issues and adult education. Perspectives from Latin America, Southern Africa and the United States (pp. 387-398). San Francisco: JosseyBass.

Fenwick, T. (2003). Learning through experience: Troubling orthodoxies and intersecting questions. Malabar, FL: Krieger.

Ferraro, J. M. (2000). Reflective practice and professional development (ERIC Digest ED449120). Washington, DC: ERIC Clearinghouse on Teaching and Teacher Education. http://www.ericfacility.net/ericdigests/ed449120.html

Finger, M. (2005). Globalization. In L. M. English (Ed.), International encyclopedia of adult education (pp. 269-273). London: Palgrave Macmillan.

Flannery, D. (1994). Changing dominant understandings of adults as learners. In E. Hayes, \& S. Colin (Eds.), Confronting racism and sexism in adult education. New directions for adult and continuing education, 61, 17-26.

Fröberg Idling, P. (2009). De glimlach van Pol Pot. Amsterdam: Nieuw Amsterdam uitgevers.

Fullan, M. (2001). The new meaning of educational change. New York: Teachers College Press.

Gervedink Nijhuis, C. J. (2008). Impact of culture on curriculum design in Ghana. Paper presented at the annual meeting of the American Educational Research Association, New York City, March 24-28, 2008.

Glastra, F. J., Hake, B. J., \& Schedler, P. E. (2004). Lifelong learning as transitional learning. Adult Education Quarterly, 54(4), 291-307.

Godfrey, M., et. al. (2000). Technical assistance and capacity development in an aid-dependent economy: The experience of Cambodia. Phnom Penh: Cambodia development resource institute.

Grindle, M. S. (Ed.). (1997). Getting good government. Capacity building in the public sectors of developing countries. Harvard Institute for International Development, Harvard University.

Groener, Z. (2006). Adult education and social transformation. In S. B. Merriam, B. C. Courtenay, \& R. M. Cervero (Eds.), Global issues and adult education. Perspectives from Latin America, Southern Africa and the United States (pp. 5-14). San Francisco, CA: Jossey-Bass.

Guskey, T. R. (2000). Evaluating professional development. Thousand Oaks: Corwin Press.

Guy, T. C. (1999a). Culture as a context for adult education: The need for culturally relevant adult education. New directions for adult and continuing education, 82, 5-18. 
Guy, T. C. (1999b). Culturally relevant adult education: Key themes and common purposes. New Directions for Adult and Continuing Education, 82, 93-98.

Hailey, J. (2001). Beyond the formulaic: Process and practice in South Asian NGOs. In B. Cooke, \& U. Kothari (Eds.), Participation. The new tiranny? (pp. 88-101). London/New York: Zed Books.

Handal, G. (1991). Promoting the articulation of tacit knowledge through the counselling of practitioners. In H. K. Letiche, J. C. van der Wolf, \& F X. Plooij (Eds.), The practitioner's power of choice in staff development and in-service training. Lisse: Swets and Zeitlinger B. V.

Harmer, A. (1995). Rebuilding war-torn societies: Psycho-social vulnerability and coping mechanisms in Cambodia. A social research feasibility study.

Heron, J. (1999). The complete facilitator's handbook. London: Kogan Page Publishers.

Hilderbrand, M. E., \& Grindle, M. S. (1997). Building sustainable capacity in the public sector: What can be done? In M. S. Grindle, (Ed.), Getting good government. Capacity building in the public sectors of developing countries (pp. 31-62). Boston, MA: Harvard Institute for International Development, Harvard University.

Hofstede, G. (1980). Culture's consequences: International differences in work-related values. Beverly Hills: Sage.

Hofstede, G. (1986). Cultural differences in teaching and learning. International Journal of Intercultural Relations, 10, 301-320.

Hofstede, G., \& McCrae, R. R. (2004). Personality and culture revisited: Linking traits and dimensions of culture. Cross-Cultural Research, 38, 52-88.

Holton, E. F., Bates, R. A., Ruona, W. E. A., \& Leimbach, M. (1998). Development and validation of a generalized learning transfer climate questionnaire final report. In R. J. Torraco (Ed.), Proceedings of the annual conference of the Academy of Human Resource Development, Oak Brook.

Homan, T. H. (2005). Leren samenwerken faciliteren. Opleiding \& Ontwikkeling, Tijdschrift voor Human Resource Development, 18(3), 17-21.

Houle, C. O. (1988). The inquiring mind (2nd ed.). Madison: University of Wisconsin, Press \& Norman.

Huber, G. L., \& Roth, J. H. W. (1999). Finden oder suchen? Lehren und lernen in Zeiten der Ungewissheit. Schwangau: Ingeborg Huber.

Humbert-Hesse, M. (1923). Rapport generale sur l'enseignement au Cambodge, 10 Janvier 1923. AOM RSC 304.

Illeris, K. (2004). Adult education and adult learning. Malabar, FL: Krieger.

James, C. R. (2002). Designing learning organizations. Organizational Dyamics, 32(1), 46-61.

Jaycox, E. V. K. (1993). Capacity building: The missing link in African development. Transcript of speech presented to the Conference on African capacity building: Effective and enduring partnerships at the African-American institute, May 20, Reston, VA. Cited from M. S. Grindle (Ed.), Getting good government. Capacity building in the public sectors of developing countries. Boston, MA: Harvard Institute for International Development, Harvard University. 
JICA (2008). Cambodia science teacher education project (STEPAM2). Phnom Penh: Ministry of Education, Youth and Sports.

Joyce, B., Bush, R., \& McKibbin, M. (1982). The California staff development study. The January 1982 report. Palo Alto: Booksend Laboratories.

Joyce, B., \& Showers, B. (2002). Student achievement through staff development. Alexandria, VA: ASCD.

Junne, G., \& Verkoren, W. (2005a). The challenges of postconflict development. In G. Junne, \& W. Verkoren (Eds.), Postconflict development: Meeting new challenges (pp. 1-18).

Junne, G., \& Verkoren, W. (2005b). Seeking the best way forward. In G. Junne \& W. Verkoren (Eds.), Postconflict development: Meeting new challenges (pp. 307-326).

Kearsley, G., \& Schneiderman, B. (1999). Engagement theory: A framework for technology-based teaching and learning. Http://home.sprynet.com/ gkearsley/engage.htm

Kelly, A. E. (2006). Quality criteria for design research: Evidence and commitments. In J. van den Akker, K. Gravemeijer, S. McKenney, \& N. Nieveen (Eds.). Educational design research (pp. 107-118). London: Routledge.

Kessels, J. W. M. (1993). Towards design standards for curriculum consistency in corporate education. Enschede: Twente University.

Kessels, J. W. M. (1996). Het corporate curriculum. Leiden: Rijks Universiteit Leiden.

Kessels, W. M., \& Keursten, P. (2001). Opleiden en leren in een kenniseconomie: Vormgeven van een corporate curriculum. In J. W. M. Kessels, \& R. F. Poell (Eds.), Human resource development. Organiseren van het leren (pp. 5-20). Alphen aan den Rijn: Samsom.

Knowles, M. S. (1980). The modern practice of adult education: From pedagogy to andragogy (2nd ed.). New York: Cambridge Books.

Knowles, K. G. (1990). The adult learner: A neglected species. Houston, Texas: Gulf Publishing.

Kolb, D. A. (1984). Experiential learning: Experience as the source of learning and eevelopment. Englewood Cliffs, NJ: Prentice Hall.

Kroehnert, G. (1991). 100 training games. Sydney: McGraw-Hill Book Company.

Kwakman, C. H. E. (1999). Leren van docenten tijdens de beroepsloopbaan. Studies naar professionaliteit op de werkplek in het voorgezet onderwijs. Thesis Katholieke Universiteit Nijmegen.

Livingstone, C. (1996). Gecko tails: Journey through Cambodia. Quezon City: Phoenix Publishing House.

Lowyck, J. (2001). Ontwerpen van leertrajecten. In J. W. M. Kessels, \& R. F. Poell (Eds.), Human resource development: Organiseren van leren (pp. 165-180). Deventer: Kluwer.

Malvicini, P. G. (2006). Popular education and lifelong learning. An integrative approach to poverty, development, and change. In Merriam, S. B., Courtenay, B. C., \& R. M. Cervero, Global issues and adult education. Perspectives from Latin America, Southern Africa and the United States (pp. 443-454). San Francisco, CA: Jossey-Bass.

Marchisani, L., \& Adams, M. (1992). Promoting diversity in college classrooms. San Francisco, CA: Jossey-Bass. 
Marsick, V. J., \& Watkins, K. E. (1997). Lessons from informal and incidental learning. In J. Burgoyne, \& M. Reynols (Eds.), Management learning: Integrating perspectives in theory and practice (pp. 295-311). Thousand Oaks, CA: Sage.

McKenney, S., Nieveen, N., \& Van den Akker, J. (2006). Design research from a curriculum perspective. In J. van den Akker, K. Gravemeijer, S. McKenney, \& N. Nieveen (Eds.), Educational design research (pp. 67-90). London: Routledge.

McNamara, V. (1999). The education system of Cambodia: National context and development issues. In D. Sloper (Ed.), Higher education in Cambodia. The social and educational context for reconstruction (pp. 99-120). Bangkok: UNESCO.

Merriam, S. B., Courtenay, B. C., \& Cervero, R. M. (2006). Global issues and adult education. Perspectives from Latin America, Southern Africa and the United States. San Francisco, CA: Jossey-Bass.

Merriam, S. B., Caffarella, R. S., \& Baumgartner, L. M. (2007). Learning in adulthood, a comprehensive guide. San Francisco, CA: Jossey-Bass.

Merriam, S. B., \& Ntseane, G. (2008). Transformational learning in Botswana: How culture shapes the process. Adult Education Quarterly, 58(3), 183-197.

MoEYS. (2005a). Education sector support program 2006-2010. Phnom Penh: MoeYS.

MoEYS. (2005b). Website: http://www.moeys.gov.kh/en/about_moeys/org_chart.htm

Mutz, G., \& Benda, N. (2005). Civil society in the Mekong region. Südostasien aktuell - Journal of Current Southeast Asian Affairs, 24(3), 16-20.

NEFAC, (2003). Education for all national plan 2003-2015. Phnom Penh: Secretariat General of NEFAC.

$\mathrm{Ng}$, E., \& Bereiter, C. (1991). Three levels of goal orientation in learning. The Journal of Learning Sciences, 1, 243-271.

Nieveen, N. (2009). Educational design research: An introduction. In T. Plomp, \& N. Nieveen (Eds.), An introduction to educational design research (pp. 9-36). Enschede: SLO.

O'Leary, M. (2007). Values in development practice, 4. Putting values into practice. Phnom Penh: VBNK.

Patton, M. Q. (1990). Qualitative evaluation and research methods. Newbury Park, Sage.

Peterse, L., \& Petri, J. (2004). Cambodja. Haarlem: Gottmer Uitgevers Groep.

Peterson, S. B. (1997). Hierarchy versus networks. Alternative strategies for building organizational capacity in public bureaucracies in Africa. In M. S. Grindle (Ed.), Getting good government. Capacity building in the public sectors of developing countries (pp. 157-176). Boston, MA: Harvard Institute for International Development, Harvard University.

Piaget, J. (1952). The origins of intelligence in children. New York: International Universities Press.

Pinto, D. (2000). Een nieuw perspectief. Herziening van beleid, onderwijs, communicatie, maslowpyramide dringend nodig. Amsterdam: Vossiuspers AUP. 
Poell, R. F. (1998). Organizing work-related learning projects: A network approach. Thesis, Katholieke Universiteit Nijmegen.

Poell, R. F., \& van der Krogt (1999). Leerstrategieën van werknemers in de kenniscreërende organisatie: Van kennisdragers naar actoren in netwerken. In J. M. Dekker, J. W. M. Kessels, C. A. Smit, \& A.N. Papas-Talen (Eds.), Netwerkend leren: Opleiders in organisaties/Capita Selecta, 38 (pp. 81-98). Deventer: Kluwer.

Pratt, D. D., Kelly M., \& Wong, W. (1998). The social construction of Chinese models of teaching. AERC Proceedings. University of British Columbia, Canada. http://www.edst.educ.ubc.ca/aerc/1998/98pratt.htm

Pratt, D. D. (1992). Chinese conceptions of learning and teaching: A Westerner's attempt at understanding. International Journal of Lifelong Education, 11(4), 301-319.

Risse, T., \& Sikkink, K. (1999). The socialization of international human rights norms into domestic practices: Introduction. In T. Risse, S. C. Ropp, \& K. Sikkink (Eds.), The power of human rights. International norms and domestic change (pp. 1-38). Cambrigde: Cambrigde University Press.

Rondeel, M., \& Wagenaar, S. (2001). Van kennismanagement naar kennisproductiviteit: Een actueel overzicht van benaderingen in de literatuur. In J. W. M. Kessels, \& R. F. Poell (Eds.), Human resource development. Organiseren van het leren (pp. 21-36). Alphen aan den Rijn: Samsom.

Schmidt, M. R. (2000). You know more than you can say: In memory of Donald A. Schon (1930-1997). Public Administration Review, 60(3), 266-274.

Schon, D. A. (1967). Technology and change: The new heraclitus. New York: Dell Publishing.

Schon, D. A. (1971). Beyond the stable state: Public and private learning in a changing society. London: Temple Smith.

SCW. (2006). The atlas of Cambodia; National poverty and environment maps. Phnom Penh: SCW.

Siddiqui, F., Strickler, C., \& Vinde, C. (2004). Capacity building practices of Cambodia's development partners. Results of a survey. Phnom Penh: CDC.

Skule, S. (2004). Learning conditions at work: A framework to understand and assess informal learning in the workplace. International Journal of Training and Development 8(1), 8-17.

Smith, M. K. (2001). Donald Schön: Learning, reflection and change, the encyclopedia of informal education. www.infed.org/thinkers/et-schon.htm

Spiecker, B. (1991). Indoctrination: The suppression of critical dispositions. In B. Spiecker, \& R. Straughan, Freedom and indoctrination in education. International perspectives. (pp. 16-29). London: Cassell Education.

Stewart, T. A. (1998). Intellectueel kapitaal. Schoonhoven: Academic Service.

Taba, H. (1962). Curriculum development: Theory and practice. New York: Harcourt, Brace \& World.

Tat, P. (2006). Training needs assessment. Phnom Penh: ITMS. 
Taylor, C., Wheeler, T., \& Robinson, D. (1996). Cambodia. Travel survival kit. Hawthorn: Lonely Planet.

Thijssen, J. G. L. (1996). Leren, leeftijd en loopbaanperspectief. Thesis, Deventer: Kluwer.

Thion, S. (1993). Watching Cambodia: Ten paths to enter the Cambodian tangle. Bangkok: White Lotus.

Tjepkema, S. (2001). Leren binnen zelfsturende teams. In J. W. M. Kessels, \& R. F. Poell (Eds.), Human resource development. Organiseren van het leren (pp. 255-270), Alphen aan den Rijn: Samsom.

Tomassini, M. (2001). New meanings of learning organisations and spaces of human resources development within the globalising learning economy. First Conference on HRD research and practice across Europe, Conference papers, Kingston: Kingstons Business School. In J. W. M. Kessels, \& R. F. Poell (Eds.), Human resource development. Organiseren van het leren (pp. 47). Alphen aan den Rijn: Samsom.

Tully, J. (2002). France on the Mekong. A history of the protectorate in Cambodia, 1863-1953. Lanham, MD: University Press of America.

UNDP, (2008). Human development indices: A statistical update 2008 - HDI ranking. www.hdr/undp.org/en/statistics

UNESCO, (2005). CTC manual. Bangok: UNESCO.

Valentine, T., \& Darkenwald, G. G. (1990). Deterrents to participation in adult education: Profiles of potential learners. Adult Education Quarterly, 4(1), 29-42.

van den Akker, J. (1998). De uitbeelding van het curriculum. Enschede: Universiteit Twente.

van den Akker, J. (1999). Principles and methods of development research. In J. van den Akker, R. M. Branch, K. Gustafson, N. Nieveen, \& T. Plomp (Eds.), Design approaches and tools in education and training (pp. 1-14). Dordrecht: Kluwer Academic Publishers.

van den Akker, J. (2003). Curriculum perspectives: An introduction. In J. van den Akker, W. Kuiper, \& U. Hameyer (Eds.), Curriculum landscapes and trends (pp. 1-10). Dordrecht: Kluwer Academic Publishers.

van de Poll, R. A. (2005). Afspraak is afspraak? Samenwerken in Wit-Rusland en Egypte. Opleiding \& Ontwikkeling, Tijdschrift voor Human Resource Development, 18(3), 22-26.

van der Linden, J., \& Roelofs, E. (2000). Leren in dialoog. Groningen: Wolters-Noordhof.

van der Sanden, J. (2001). Opleiden vanuit een constructivistisch perspectief. In J. W. M. Kessels, \& R. F. Poell (Eds.), Human resource development. Organiseren van het leren (pp. 5366). Alphen aan den Rijn: Samsom.

van Eijbergen, R. (2005). Misschien wel het belangrijkst wat je kunt doen als adviseur. Opleiding \& Ontwikkeling, Tijdschrift voor Human Resource Development, 18(3), 9-12.

van Lakerveld, J. (2005). Het corporate curriculum. Onderzoek naar werk-leeromstandigheden in instellingen voor zorg en welzijn. Thesis, Enschede: University of Twente.

Verkoren, W. (2005). Bringing it all together: A case study of Cambodia. In G. Junne, \& W. Verkoren (Eds.), Postconflict development: Meeting new challenges (pp. 289-306). 
von Krogh, G., \& Roos, J. (Eds.) (1996). Managing knowledge: Perspectives on cooperation and competition. London: Sage publications.

Vygotsky, L. (1978). Mind and society: The development of higher psychological processes. Cambridge, MA: Harvard University Press.

Weggeman, M. (2000). Kennismanagement: De praktijk. Schiedam: Scriptum Management.

Werner, M. (2001). Human resource management in the People's Republic of China. In P. S. Budhwar, \& Y. A. Debrah, (Eds.), Human resource management in developing countries (pp. 91-101). London: Routledge.

Witziers, B. (2001). Het effectiviteitsvraagstuk rondom bedrijfsopleidingen. In J. W. M. Kessels, \& R. F. Poell (Eds.), Human resource development. Organiseren van het leren (pp. 385-398). Alphen aan den Rijn: Samsom.

Wood, G. T., \& Mellahi, K. (2001). Human resource management in South Africa. In P. S. Budhwar, \& Y. A. Debrah (Eds.). Human resource management in developing countries (pp. 222-237). London: Routledge.

Wright, A. R. (1997). Participation, ownership, and sustainable development. In M. S. Grindle (Ed.), Getting good government. Capacity building in the public sectors of developing countries (pp. 369-412). Boston, MA: Harvard Institute for International Development, Harvard University.

Yin, R. K. (2003). Case study research: Design and methods. Thousand Oaks, CA: Sage. 


\section{ENGLISH SUMMARY}

\section{INTRODUCTION}

Over the past 10 years, the Cambodian Ministry of Education, Youth and Sports (MoEYS), has been trying to build the capacity of its staff after decades of civil war and a near annihilation of its educated elite. With the help of international aid organisations, numerous professional development activities (PDAs) have been executed. The results of these programs vary, but in general the outcomes can be classified as weak. These weak outcomes are partly a result of the initial absence of any education system, followed by the establishment of an education system that was weak. In most cases, neither students, nor Government staff, are trained in either high-level subject matter or in academic and meta-cognitive skills. Furthermore, the quality of the PDAs varies. And finally, the Ministry lacks a learning-supporting Human Resource Management (HRM) system, offering no career opportunities or incentives for developing staff. Despite these constraints, the Ministry and its staff are eager to improve their performance. At present, selected Ministry staff members are responsible for capacity building of colleagues at central level, and also of staff at provincial, district and school levels. Aforementioned poor quality of the training courses and workshops provided is caused by poor facilitation and a lack of participating and learnerbased methodologies. Often, only theory is presented, and practice and follow-up support are, on the whole, non-existent. The outcomes of these endeavours, in the sense of application in the workplace, are negligible. 


\section{AIM AND RESEARCH QUESTIONS}

MoEYS acknowledged the poor outcomes of its training endeavours. The research described in this dissertation was conducted at the request of the Directorate General of Education (DGE) of MoEYS. It aimed at strengthening training organisation and facilitation skills of staff in all Basic Education Departments residing under the DGE. It also tried to identify the characteristics of a potentially more effective and participatory professional development scenario, thereby extending the existing knowledge base on professional development and learning in Cambodia. The following research question and sub-questions formed the basis of the research:

What characteristics should an effective professional development scenario have, when its aim is to strengthen the training facilitation skills of Ministry of Education staff in Cambodia?

And the following sub-questions:

1. Which principles for the design of such a scenario can be derived from a context analysis and a literature review?

2. What are the effects of such a scenario on the acquisition and utilisation of knowledge, skills and attitudes by the staff members?

3. To what extent is the learning of the staff members influenced by (organisational) culture?

The outcomes of the research did not only lead to the scenario described in this thesis, but also to a Training Guideline document for MoEYS and tools to improve training (checklists for training organisation and evaluation forms).

\section{RESEARCH APPROACH AND DESIGN}

An educational design research approach was chosen for the conduct of the research described in this dissertation. Educational design research can be applied for the development of products and interventions in complex, dynamic and poorly understood contexts. The Cambodian context met these requirements. This form of research aims at developing practical interventions in existing 'real world' settings. It tries to do so by adopting an iterative (cyclic) process of design, 
evaluation and revision. During the process, contextual understanding is developed and this understanding contributes to the knowledge base about the context in which the educational design research is performed.

The research was conducted in three phases. During the preparatory phase, a context analysis, a literature review and an analysis of needs and requirements of MoEYS were conducted. The preparatory phase led to the formulation of design principles. These design principles were used during the design and implementation phase to develop an initial scenario design that evolved into the enacted scenario. During the enacted scenario, an interdepartmental working group, or community of learners, consisting of nine staff members was established. During a period of six months, this community of learners not only developed a Training Guideline with instruments, but also improved its understanding of professional development and its training skills. The Training Guideline was introduced during a four-day Training Guideline training for MoEYS staff half a year later. The working group worked collaboratively following a 'learning by designing' approach based on socio-constructivism ( $\mathrm{Ng}$ \& Bereiter, 1991) and supported by a 'rich' learning environment enhancing learning as advocated by Kessels' corporate curriculum concept (Kessels, 1996). Following this approach, the group members acquired a shared conceptual framework of knowledge and a set of organisation and facilitation skills while working collaboratively on the products.

By incorporating the training components model (Joyce \& Showers, 2002), acquisition and application of knowledge and skills were reinforced. Incorporation of the training components model meant that knowledge and skills were modelled and practised during the eleven working group meetings; follow-up support was provided, while group members were experimenting with and implementing the acquired knowledge and skills during the four-day Training Guideline training as well as during training sessions and workshops conducted at the workplace. Micro-cycles were incorporated into the working group meetings activities. These micro-cycles followed iterative cycles of design, testing and revision. For example, the checklists and evaluation forms were developed, but also the definitions and concepts of professional development that together formed the contextualised conceptual framework of professional development were constructed with microcycles. A safe and supportive learning environment was established in order to accommodate successful collaborative and participatory learning. 


\section{RESULTS}

The quality of the enacted scenario was evaluated on its relevance, consistency, practicality and effectiveness (Nieveen, 2009; van den Akker, 1999). The curricular spider web (van den Akker, 2003), the critical levels of professional development evaluation (Guskey, 2000), and the 5-D model for cultural differences (Hofstede, 1986; Hofstede \& McCrae, 2004; Gervedink Nijhuis, 2008) were used to assess these criteria.

The scenario was relevant, as it was developed based on the articulated needs of the Ministry and the community of learners. It was conducted within the mandate provided by MoEYS. His Excellency the Director General and the Deputy Director of the DGE fully supported the scenario, which was important for the status of the scenario. However, the working group never gained formal status. A formal status in the form of a stamped and signed letter by a highranking MoEYS official would have made it easier for group members to allocate time to join the meetings and not be called away to other endeavours.

The scenario was also quite consistent, as most of the components of the curricular spider web were consistent with each other. The provided materials supported the learning activities, and grouping was consistent with the activities. But location and time were not consistent with the rest of the scenario. Meeting rooms were not supportive towards participatory and learner-based methodologies, although that did not seem to cause concern to the learners. In general, they were very flexible and co-operated with the activities, although the environment was less suitable. Time was the biggest constraint, with high absenteeism on the part of some learners having a negative effect on their personal learning outcomes.

The practical usability of the developed materials like the Training Guideline document and most of its tools was high. The iterative micro-cycle approach for the development of these tools was appropriate. The adopted 'learning by designing' approach with its incorporated components of theory, modelling, practice and follow-up, was also appropriate for improving knowledge and skills acquisition and application. But the effectiveness of the scenario was highly influenced by the personal commitment of the learners and the amount of support offered to them by their superiors. 
Based on the research presented in this thesis, a typology of facilitators' levels was developed, from novice educator to artisan facilitator and catalyst of learning levels. Of the nine group members, two developed into 'artisan facilitators', three became 'mechanical facilitators' and four did not advance beyond the 'educator' levels.

The most successful learners progressed to the level of 'artisan facilitators', meaning that they were able to adapt learning activities to different contexts. They were highly motivated to experiment with the acquired knowledge and skills, and were offered the opportunities to do so by their Department Directors. The learners who progressed to the 'mechanical facilitation' level were not yet able to adapt activities to the context. They were mostly present during the working group meetings, but had fewer opportunities, or were more reluctant to practise and experiment with the acquired knowledge and skills. The least successful members were often absent during the working group meetings, as a result of weak support from their superiors. They were often called away to other meetings and were not offered opportunities to practise and experiment with the acquired knowledge and skills. The reduced commitment of some of the least successful learners further contributed to the low learning results. Their high absenteeism limited their engagement with the important modelling, practising and supporting components of the scenario, and involvement with the microcycles. Because they failed to fully engage with the entire scenario, they did not progress beyond the 'experienced educator' level.

Both local (organisational) culture and history played a large role in learning during the scenario, and as it possibly does in all learning in Cambodia. Trust is an important prerequisite for collaborative learning, but trust has been heavily violated in the recent history of the country. Establishing a safe learning environment, in which people felt free to express their opinions, was essential. But freedom of expression was also affected by large power distance and hierarchical relationships between learners. Furthermore, high uncertainty avoidance further complicated the possibility of an open exchange of ideas, on one hand, and limited willingness or ability to practise with new methodologies, on the other. This research shows that it is indeed possible to empower people to work collaboratively together within the Cambodian context, but that activities and approaches need to be contextualised in order to arrive at successful outcomes.

Academic, collaborative, communication and meta-cognitive skills are important in order to arrive at effective collaborative learning. The scenario was able to strengthen and contextualize collaborative and communication skills. On the 
other hand, building academic and meta-cognitive skills remained extremely difficult. The historical and cultural context is not supportive towards the development of these important skills. The scenario was not able to achieve significant change in the level of these skills.

The cognitive apprenticeship model (Holton, Bates, Ruona \& Leimbach, 1989) was an effective model for the support of the learning of group members during the scenario. The model, however, is based on progressive steps towards ownership of one's own learning and does not acknowledge the cultural habit of Cambodians to ask for continuous reassurance while learning. Because of high uncertainty avoidance by some of the learners, they did not progress beyond the 'assistance-on-request' level.

\section{CONCLUSIONS}

The adoption of a collaborative 'learning by designing' approach, based on notions of the corporate curriculum as advocated by Kessels (1996) and socioconstructivist learning theory ( $\mathrm{Ng} \&$ Bereiter, 1991), has a positive effect on the professional development of Government staff in Cambodia. A learning scenario that follows such an approach should incorporate all training components of the model developed by Joyce and Showers (2002), in order to enhance acquisition and application of knowledge and skills. Iterative micro-cycles of design, testing and revision, are important to arrive at 'fine-tuned' and contextualised products and methodologies.

Members of a community of learners are not tabulae rasae, but possess contextual knowledge that is important to achieve practical outcomes of scenarios. Although learners might have acquired knowledge and skills during previous endeavours, this knowledge and skills might be poorly organised and remembered, leading to false clarity and superficial understanding (Fullan, 2001). This was also the case for the understanding of concepts of professional development with the community of learners in this research. Coming to a shared, academically grounded and contextualised conceptual framework of professional development theory was crucial as it formed the base for all further work to be carried out by the working group. It is, therefore, strongly recommended that an effort be made to establish a shared and contextual conceptual framework at the beginning of a professional development scenario. 
Time is necessary for the building of trust with and between the members of a community of learners, on the one hand, and developing thorough contextual understanding on the other. The influence of cultural dispositions like power distance, uncertainty avoidance and the importance of status can only be understood while working in a context for a prolonged period of time. International aid organisations often do not provide that time, as they strive for quick results. But contextual understanding is essential for identifying the influence of cultural issues and providing efficient support for collaborative learning in order to arrive at practical and effective outcomes of an intervention. Neglecting the cultural aspects of learning can easily lead to professional development outcomes without any long-term effects. The goal of any professional development scenario in developing countries should not be to change local and organisational cultures in order for these to fit the learning activities of these projects, but to adapt the learning activities to the local context.

Support by senior officials is, together with the allocation of adequate time, a major influence on the effect of a professional development scenario. Senior officials should allow their staff to engage in all meetings of a learning scenario and provide enough opportunities for their staff to apply the acquired knowledge and skills at the workplace. Dissemination to colleagues is important for a wider effect in the organisation. Therefore, the Government should grant a community of learners a formal status, in order to achieve the best results.

In contrast, however, with the ideas of Berman, McLaughlin and associates (1979), leaders in societies with strong hierarchical relationships, like the Cambodian one, should not be present at the meetings of working groups, but remain involved at a distance, in order to prevent the collaborative learning processes of their staff of being disturbed by their presence.

Working in a more collaborative way increases the need for learners to develop academic and meta-cognitive skills. This research shows that the acquisition of stronger academic and meta-cognitive skills is complicated in the Cambodian setting. More thorough interventions and programs are needed to stimulate the acquisition of these skills.

It is hoped that the research presented in this dissertation will contribute to more effective and collaborative professional learning in Cambodia and other countries with similar professional development issues. 


\section{NEDERLANDSE SAMENVATTING}

\section{INLEIDING}

Gedurende de afgelopen tien jaar, na decennia van burgeroorlog, heeft het Cambodjaanse Ministerie van Onderwijs, Jeugdzaken en Sport (MoEYS) geprobeerd de professionele capaciteit van haar staf te versterken. Met steun van internationale hulporganisaties zijn verschillende programma's voor professionele ontwikkeling uitgevoerd. De opbrengst van deze programma's kan in het algemeen als gering getypeerd worden. Dat is gedeeltelijk te wijten aan een eerst ontbrekend (gedurende het bewind van de Rode Khmer) en daarna falend onderwijssysteem (sinds de wederopbouw), waardoor studenten, en dus ook de Ministeriële staf, geen hoogwaardige vakinhoudelijke kennis konden opdoen, noch getraind werden in academische en metacognitieve (leerregulerende) vaardigheden. De trainingen en workshops die sinds de wederopbouw verzorgd worden om de kennis en vaardigheden op niveau te brengen, zijn van sterk wisselende kwaliteit. Tot slot mist het Ministerie een human resource management (HRM) beleid dat gericht is op het ondersteunen van leeractiviteiten. Het huidige HRM beleid biedt geen carrièremogelijkheden, noch beloningen voor stafleden die zich professioneel ontwikkelen.

Ondanks deze beperking is de ministeriële staf erop gebrand de prestaties op het gebied van professionele ontwikkeling binnen het Ministerie te verbeteren. Sinds enige jaren is de daarvoor door het Ministerie geselecteerde staf verantwoordelijk voor de capaciteitsopbouw van collega's op centraal niveau, maar ook voor de professionele ontwikkeling van de staf op provinciaal, district en schoolniveau. Het eerder genoemde niveau van trainingen en workshops wordt veroorzaakt door het matige niveau van trainingsvaardigheden en het ontbreken van participatieve en activerende (learner-based) werkvormen. In de meeste gevallen 
wordt tijdens de trainingen en workshops alleen theorie aangeboden, en ontbreken de mogelijkheden voor praktische uitvoering en follow-up ondersteuning. De opbrengst van deze pogingen om de professionele ontwikkeling te versterken is dan ook verwaarloosbaar, zeker als men kijkt naar de mate van toepassing op de werkplek.

\section{DOEL VAN HET ONDERZOEK EN ONDERZOEKSVRAGEN}

MoEYS onderkent de lage opbrengst van haar trainingstrajecten. Het hier beschreven onderzoek is uitgevoerd op verzoek van het Directoraat Generaal van Onderwijs (DGE) binnen MoEYS. Het onderzoek heeft tot doel de trainingsvaardigheden van de staf van de Departementen, die onder het DGE vallen, te versterken. Voorts tracht dit onderzoek die eigenschappen te beschrijven welke een potentieel effectiever scenario voor professionele ontwikkeling dient te hebben. Daarmee wordt gelijktijdig getracht de kennisbasis over professionele ontwikkeling en leren in Cambodja te vergroten. Daarvoor zijn de volgende onderzoeksvraag en deelvragen geformuleerd:

\section{Onderzoeksvraag:}

Welke kenmerken dient een effectief professioneel ontwikkelingsscenario te hebben, dat gericht is op het versterken van trainingsvaardigheden van stafleden van het Ministerie van Onderwijs in Cambodja?

Deelvragen:

1. Welke principes voor de ontwikkeling van een dergelijk scenario kunnen worden afgeleid uit de context analyse en een exploratie van de literatuur?

2. Wat zijn de effecten van een dergelijk scenario op de verwerving en toepassing van kennis, vaardigheden en houdingen van stafleden?

3. In welke mate wordt het leren van stafleden beïnvloed door (organisatie) cultuur?

De uitkomsten van dit onderzoek hebben niet alleen geleid tot deze dissertatie, maar ook tot een Training Guideline document, en de daarbij ontwikkelde instrumenten om training te verbeteren (checklists voor het organiseren van training en formulieren om training te evalueren). 


\section{ONDERZOEKSAANPAK}

Voor dit onderzoek is een aanpak gekozen die beschreven is als educational design research (onderwijskundig ontwerp onderzoek). Deze vorm van onderzoek wordt toegepast voor de ontwikkeling van producten en scenario's binnen complexe, dynamische en matig begrepen contexten. De Cambodjaanse context voldoet aan deze criteria. Ontwerp onderzoek tracht praktische interventies te ontwikkelen die toepasbaar zijn binnen bestaande omgevingen. Het probeert dat te doen door het volgen van een iteratief ontwikkelingsproces waarin ontwerp, evaluatie en revisie elkaar steeds opvolgen. Gedurende het proces wordt gelijktijdig de context steeds beter begrepen, waardoor het onderzoek bijdraagt aan het uitbreiden van de kennisbasis over de onderzochte context en aan de manier waarop ontwerp onderzoek in een dergelijke context kan worden toegepast.

Het hier beschreven onderzoek is uitgevoerd in drie fasen. Gedurende de voorbereidingsfase zijn een contextanalyse, een literatuuranalyse en een analyse van de wensen op het gebied van professionele ontwikkeling binnen het Ministerie uitgevoerd. Aan het einde van deze eerste fase konden ontwerpprincipes geformuleerd worden, die in de ontwerp/ontwikkel- en implementatiefase zouden leiden tot een eerste ontwerp van het beoogde scenario. Dit eerste ontwerp evolueerde vervolgens gedurende de rest van de fase tot het uitgevoerde of enacted scenario. Het scenario ging van start met het instellen van een interdepartementale werkgroep, of leergemeenschap (een community of learners), bestaande uit negen personen. Deze leergemeenschap ontwikkelde vervolgens gedurende zes maanden de Training Guideline met bijbehorende instrumenten, maar verbeterde ook het eigen begrip over professionele ontwikkeling en de persoonlijke trainingsvaardigheden. De Training Guideline werd ruim een half jaar later door middel van een vierdaagse training geïntroduceerd binnen het Ministerie. Voor het werk in de leergemeenschap werd een learning by designing (Lowyck, 2001) benadering gekozen, die gebaseerd was op de socio-constructivistische leertheorie (Ng \& Bereiter, 1991) en op noties van het corporate curriculum zoals Kessels die beschrijft (1996). In de praktijk betekende dit dat de groepsleden gezamenlijk een gedeeld begrippenkader en een set trainingsvaardigheden ontwikkelden waardoor ze tegelijkertijd hun eigen kennis en vaardigheden vergrootten.

Door het inbouwen van het training component model (Joyce \& Showers, 2002) in het scenario werden verwerving en toepassing van kennis en vaardigheden versterkt. Dit betekende in de praktijk dat kennis en vaardigheden door middel 
van theorie, modelleren, het aanbieden van mogelijkheden tot het in de praktijk brengen van het geleerde, werden aangeboden. Het in de praktijk brengen werd ondersteund door middel van follow-up, de belangrijkste stap van het model. Daarnaast werden groepsleden die buiten het scenario om met kennis en vaardigheden wilden experimenteren, ondersteund.

Voorts werd door middel van opeenvolgende fasen van ontwerpen, testen en bijstellen (microcycli) tijdens de groepsbijeenkomsten getracht om tot toegespitste en gecontextualiseerde opbrengsten te komen. Voorbeelden daarvan zijn de checklists en evaluatieformulieren. Ook de definities en concepten die onderdeel zijn van het ontwikkelde gecontextualiseerde conceptuele raamwerk voor professionele ontwikkeling, zijn door middel van microcycli tot stand gekomen. Een veilige en ondersteunende leeromgeving was essentieel voor de collaboratieve en participatieve aanpak van het scenario.

\section{RESULTATEN}

De kwaliteit van het ontwikkelde scenario is geëvalueerd op relevantie, consistentie, praktische bruikbaarheid en effectiviteit (Nieveen, 2009; van den Akker, 2003). Daarvoor werden het curriculaire spinnenweb (van den Akker, 2003), de vijf kritische evaluatieniveaus van professionele ontwikkeling (Guskey, 2000) en het 5-dimensionale model voor culturele verschillen (Hofstede, 1986, Hofstede \& McCrae, 2004; Gervedink Nijhuis, 2008) gebruikt.

Het scenario bleek relevant, in die zin dat het tegemoet kwam aan de leerwensen van zowel het Ministerie, als de groepsleden. Voorts is de interventie uitgevoerd binnen het door MoEYS gegeven mandaat. De Directeur Generaal en de Adjunct Directeur van het DGE steunden het scenario, wat belangrijk was voor het aanzien ervan binnen het Ministerie. De werkgroep heeft echter nooit een officiële status gekregen. Een officieel document met handtekening en stempel van een hoog staflid zou de aanwezigheid van de groepsleden tijdens de bijeenkomsten van de werkgroep zeker hebben verhoogd.

Het scenario was gedeeltelijk consistent. De meeste componenten van het curriculaire spinnenweb waren met elkaar in samenhang. Zo ondersteunden de ontwikkelde materialen de leeractiviteiten en waren de groepsvormen consistent met de leeractiviteiten. Maar de locatie- en tijdcomponenten vertoonden minder 
samenhang met de rest van de onderdelen. Zo was de vergaderruimte waarin de werkgroep samenkwam niet geschikt voor participatieve en activerende werkvormen, hoewel dit de bereidheid van de groepsleden om deze werkvormen te gebruiken niet wezenlijk lijkt te hebben beïnvloed. Over het algemeen waren de groepsleden erg flexibel tijdens het uitvoeren van de leeractiviteiten. De afwezigheid van sommige groepsleden tijdens de bijeenkomsten waarin het scenario verder ontwikkeld werd, was de meest beperkende factor. Sommige groepsleden waren zo vaak afwezig dat dit een negatieve weerslag had op hun persoonlijke leeropbrengsten.

De praktische toepasbaarheid van de ontwikkelde materialen zoals het Training Guideline document en de meeste van de bijbehorende instrumenten was hoog. De iteratieve microcyclus aanpak die gebruikt was voor de ontwikkeling van deze materialen bleek geschikt te zijn. Dit geldt ook voor de toegepaste learning by designing aanpak. De opeenvolging van het aanbieden van theorie, het modelleren, het bieden van mogelijkheden voor praktische toepassing en de geboden ondersteuning daarbij, bleek succesvol. De effectiviteit van het scenario werd enerzijds sterk beïnvloed door de mate van persoonlijke toewijding van de groepsleden en anderzijds door de mate van steun die de groepsleden kregen van hun managers. Sommige managers beperkten de mogelijkheden van de groepsleden om bijeenkomsten van de werkgroep bij te wonen.

Van de negen leden van de werkgroep ontwikkelden twee leden zich tot artisan facilitators, drie tot mechanical facilitators en vier tot het niveau van educators. De meest succesvolle groepsleden waren sterk gemotiveerd en experimenteerden met de opgedane kennis en vaardigheden. Zij kregen daar vaker de kans toe en werden daarbij gesteund door hun meerderen. Daardoor waren zij in staat om activiteiten te bewerken of aan te passen aan veranderende contexten.

De groepsleden die zich ontwikkelden tot het niveau van mechanical facilitators waren nog niet in staat tot het aanpassen van leeractiviteiten en voerden deze activiteiten voornamelijk mechanisch uit. Zij kregen van hogerhand minder kansen voor het experimenteren met de opgedane kennis en vaardigheden in de praktijk, of waren daar zelf terughoudend in.

Van de minst succesvolle groepsleden was slechts een enkeling minder gemotiveerd. Hoofdkenmerk was dat zij niet in staat waren om alle bijeenkomsten van de werkgroep bij te wonen, zodat zij niet het volledige leerproces konden doorlopen. Zij misten daardoor de mogelijkheden om 
praktisch te experimenteren met de kennis en vaardigheden die zij al in mindere mate door hun hoge absentie hadden opgedaan. In de meeste gevallen werd hun afwezigheid bepaald door hun meerderen, die hen niet in staat stelden alle bijeenkomsten van de werkgroep bij te wonen en de opgedane kennis en vaardigheden in de praktijk te brengen.

De (recente) Cambodjaanse geschiedenis en (organisatie) cultuur speelden een grote rol bij het leren tijdens de uitvoering van het scenario; dit is hoogstwaarschijnlijk het geval bij elk leerproces in Cambodja. Het hebben van vertrouwen in elkaar is een belangrijke voorwaarde voor collaboratief leren. Vertrouwen is in algemene zin echter op grote schaal geschonden in de recente Cambodjaanse geschiedenis. De tijdens het scenario gecreëerde veilige leeromgeving, waarbinnen mensen de vrijheid hadden zich te uiten, werd voortdurend beïnvloed door de machtsverschillen en de hiërarchische verhoudingen tussen de groepsleden. Verder bemoeilijkte een hoge onzekerheid (uncertainty avoidance) in het begin een open uitwisseling van ideeën, maar ook beperkte zij soms de bereidheid om te oefenen met nieuwe methodologieën. Dit onderzoek toont echter aan dat het zeer wel mogelijk is om mensen in staat te stellen om collaboratief te werken binnen de Cambodjaanse context, maar dat een dergelijke aanpak gecontextualiseerd moet worden om tot succesvolle opbrengsten te komen.

Academische, collaboratieve, communicatieve en metacognitieve vaardigheden zijn belangrijk om tot effectief collaboratief leren te komen (Kessels, 1996). Het ontwikkelde scenario was in staat om de collaboratieve en communicatieve vaardigheden van de groepsleden te vergroten. Het aanleren van academische en metacognitieve vaardigheden was echter minder succesvol. De historisch-culturele context heeft een negatieve invloed op de ontwikkeling van deze vaardigheden. Het scenario was niet bij machte hier grote verandering in aan te brengen.

Het cognitive apprenticeship model (Holton, Bates, Ruona \& Leimbach, 1989) bleek een effectief model voor het ondersteunen van het leren door de groepsleden. Het model is echter gebaseerd op progressieve stappen naar zelfstandigheid en hield geen rekening met de bevestiging van de juistheid van het handelen, waar de groepsleden bij voortduring om vroegen. Deze continue behoefte aan bevestiging is sterk geworteld in een hoge mate van onzekerheid en zorgde ervoor dat sommige groepsleden niet de stap konden maken naar het assistanceon-request niveau van het model. 


\section{CONCLUSIES}

Het volgen van een collaboratieve learning by designing (Lowyck, 2001) benadering heeft een positief effect op de professionele ontwikkeling van overheidsstaf in Cambodja. Een leerscenario dat een dergelijke benadering volgt, dient alle trainingscomponenten van het door Joyce en Showers (2002) ontworpen model in te bouwen, om tot een optimale verwerving en toepassing van kennis en vaardigheden in de praktijk te komen. Iteratieve microcycli zijn verder van belang om producten en methodologieën te ontwikkelen die toegespitst zijn op de context.

Leden van een leergemeenschap zijn geen tabulae rasae (onbeschreven bladeren), maar bezitten belangrijke contextuele kennis die van groot belang is om tot praktische opbrengsten van een scenario te komen. Lerenden kunnen tijdens eerdere interventies kennis en vaardigheden opgedaan hebben, maar deze kennis en vaardigheden kunnen slecht georganiseerd en onthouden zijn en tot valse duidelijkheid en oppervlakkig begrip leiden (Fullan, 2001). Dit was ook het geval binnen de leergemeenschap waarmee binnen dit onderzoek is gewerkt. Daarom was het ontwikkelen van een gemeenschappelijk, academisch gegrond en gecontextualiseerd conceptueel raamwerk, waar de rest van het werk tijdens het scenario op gebaseerd was, aan het begin van het scenario van het grootste belang.

Tijd is allereerst een kritische factor voor het ontwikkelen van vertrouwen met en tussen de leden van een leergemeenschap, maar is ook van belang voor het ontwikkelen van diepgaand contextueel begrip. De invloed van culturele disposities als machtsverschillen, onzekerheid en het belang van status binnen een gemeenschap kan alleen begrepen worden als gedurende langere tijd gewerkt wordt binnen een bepaalde context. Internationale hulporganisaties willen doorgaans echter resultaten boeken op de kortere termijn. Maar contextueel begrip is van het grootste belang om culturele invloeden te leren herkennen en de juiste ondersteuning te geven aan collaboratieve leerprocessen. Beiden zijn nodig om tot praktisch bruikbare en effectieve uitkomsten te komen. Het buiten beschouwing laten van de culturele aspecten van leren leidt slechts tot een ontwikkelingsscenario dat elk lange-termijn effect ontbeert. Het doel van een ontwikkelingsscenario mag nooit het aanpassen van de lokale cultuur aan de leeractiviteiten van een dergelijk traject zijn, maar moet er juist voor zorgen dat leeractiviteiten aangepast worden aan de lokale context. 
De steun van leidinggevenden is, samen met de factor tijd, de voornaamste factor die van invloed is op het effect van een professioneel ontwikkelingsscenario. Leidinggevenden dienen hun stafleden de kans te geven de bijeenkomsten van een werkgroep bij te wonen en de opgedane kennis en vaardigheden toe te passen op de werkvloer. Voorts is het doorgeven van opgedane kennis en vaardigheden aan collega's van belang voor een breder effect binnen de organisatie. Daarom is het hebben van een formele status van een leergemeenschap binnen het Ministerie van groot belang.

In tegenstelling tot wat Berman, McLaughlin en associates (1979) zeggen, is het in contexten met een sterk hiërarchisch karakter, zoals de Cambodjaanse, minder wenselijk dat leidinggevenden bij de bijeenkomsten van een leergemeenschap aanwezig zijn. Het is beter hen op een afstand bij het proces te blijven betrekken, omdat hun aanwezigheid het leerproces van de leergemeenschap zou kunnen belemmeren.

Het werken op een meer collaboratieve manier vergroot de behoefte aan het ontwikkelen van academische en metacognitieve vaardigheden van de groepsleden. Dit onderzoek toont aan dat de verwerving van dergelijke vaardigheden binnen de Cambodjaanse context een gecompliceerd proces is. Meer diepgaande interventies met een focus op het opdoen van deze vaardigheden zijn noodzakelijk.

Dit onderzoek hoopt een bijdrage te leveren aan effectievere en collaboratieve professionele ontwikkeling in Cambodja en andere landen met een soortgelijke context. De bevindingen van dit onderzoek en de aanbevelingen die in dit proefschrift gepresenteerd worden, hebben enerzijds tot doel verder bij te dragen aan een bredere kennisbasis over professionele ontwikkeling in Cambodja. Anderzijds wordt gehoopt dat zij de overheid en internationale organisaties prikkelen tot betere beleidsbeslissingen omtrent professionele ontwikkelingsscenario's. Voorts is de hoop dat de hier gepresenteerde bevindingen professionals ondersteunen bij het uitvoeren van meer collaboratieve leerprocessen in Cambodja en andere ontwikkelingslanden. 


\section{ANNEXE 1 \\ Directors' interview letter}

Phnom Penh, 15 February 15, 2007

Dear Madam / Sir,

For the development of the Training Guideline for the Ministry of Education, Youth and Sports, I would like to interview you about training carried out by your department.

The objectives for the interview are:

1. To explain about the Training Guideline;

2. To get an idea how training is done by your Department;

3. To get your experience for making training more effective;

4. To learn from you about how monitoring, evaluation and follow up is and should be done.

The interview would take approximately 30 minutes.

If you have any questions, please feel free to contact me.

Yours truly,

Jan Berkvens,

Capacity Building and education adviser, MoEYS-DGE.

$092-945910$

janberkvens@gmail.com 


\section{ANNEXE 2 \\ Directors' interview questionnaire}

\section{Questionnaire for Department Director}

Name:

Date:

Department:

\section{Training Guideline}

\begin{tabular}{|l|l|l|l|}
\hline 1 & $\begin{array}{l}\text { Does your Department have a guideline or manual on how to } \\
\text { develop and provide training? (ask for copy) }\end{array}$ & yes & no \\
\hline 2 & $\begin{array}{l}\text { Does you Department have a report format for training } \\
\text { evaluations? (ask for copy) }\end{array}$ & & \\
\hline 3 & Is a Training Guideline important for your Department? & \\
\hline
\end{tabular}

4. What should a Training Guideline contain to be useful for your Department?

\section{Training in your Department}

\begin{tabular}{|l|l|l|l|l|l|}
\hline & & Poor & Fair & Good & Excellent \\
\hline 5 & $\begin{array}{l}\text { How effective is training provided to your } \\
\text { staff? }\end{array}$ & & & & \\
\hline 6 & $\begin{array}{l}\text { How effective is training provided by your } \\
\text { staff? }\end{array}$ & & & & \\
\hline
\end{tabular}

7. How could training be made more effective?

8. What are the three most common activities in training? 
Staff

9. What is the role of ... in your Department?

\begin{tabular}{|l|l|}
\hline Trainer & \\
\hline Facilitator & \\
\hline
\end{tabular}

\begin{tabular}{|l|l|l|l|l|l|}
\hline 10. & $\begin{array}{l}\text { Hoor } \\
\text { Heveod is your staff in training } \\
\text { devent? }\end{array}$ & Fair & Good & excellent \\
\hline 11. & $\begin{array}{l}\text { How good is your staff in training } \\
\text { delivery? }\end{array}$ & & & & \\
\hline
\end{tabular}

12. What does your staff need to learn about the development and the delivery of training?

13. How could the capacity of your staff be strengthened on the development and the delivery of training?

\section{Monitoring, Evaluation and Follow up}

14. What do the following terms mean in your Department?

15. What is the order of the three?

\begin{tabular}{|l|l|l|}
\hline monitoring & & \\
\hline evaluation & & \\
\hline Follow up & & \\
\hline
\end{tabular}

16. Which methods are used for monitoring, evaluation and follow up?

17. How could monitoring, evaluation and follow up be approved?

\begin{tabular}{|l|l|l|}
\hline monitoring & & \\
\hline evaluation & & \\
\hline Follow up & & \\
\hline
\end{tabular}


Evaluation forms

The Training Guideline Working Group has developed two forms for evaluation:

1. Short Term Evaluation Form

2. Long Term Evaluation Form

Based on research, it is recommended to evaluate training not only at the end of training, but also after a few weeks or months. The Long Term Evaluation Form is designed for this purpose. It gives information on the implementation of the knowledge and skills of the course in the work of the trainee.

18. How could a Long Term Evaluation be organized in training that is provided by your Department?

Thanks for your cooperation. 


\title{
ANNEXE 3 \\ The DGE Training Guideline
}

\author{
Introduction \\ The Capacity Building Midterm Plan 2006-2010 states that the Directorate General of Education \\ will develop a training guideline that will improve the effectiveness of professional \\ development of the staff. In most cases training and workshops are delivered to the staff of \\ departments, POE's, DOE's, clusterschools, schools and communties to build the capacity of \\ participants. Until now there is not a clear structure of how trainings and workshops should be \\ developed and delivered to be as effective as possible. With the development of this Training \\ Guideline, DGE aims to design a tool that could be used by all departments at all levels in the \\ educational system to improve the skills, knowledge, behaviour and beliefs of its staff members \\ on how to develop and deliver professional development through training and workshops.
}

Although we know that training and workshops do not always give the result we hoped for, research on why this is, seems to focus only on the content of the course, participant behaviour or external reasons. The instrument of training itself is almost never investigated. This research to develop a training guideline aims to offer knowledge and skills on how to create an optimal and effective training or workshop, whatever the content.

\section{Research at the same time}

As staff members of DGE and the departments residing under the DGE have a lot of experience about developing and conducting training and workshops, we would like to use this expertise for making a study on how training and workshops are developed and conducted now. What parts are carried out with confidence and positive effects? What parts could be strengthened? Can we put together a list of advise and plans of actions on which we agree that have positive effects on the professional development of our staff?

Working in this way, building the training guideline is capacity building in itself, strengthening the knowledge, skills, behaviour and beliefs of the staff involved with writing the training guideline.

\section{Methodology}

At the start of the research, a rapid prototyping procedure is carried out, in which a training is developed, to gather information about what the group finds key issues on developing and conducting a training. This information will be used for the next step, which is testing these techniques in the field. This will be on the project management cycle, one of the other cross cutting capacity building needs. After the rapid prototyping, an overview of key training techniques will be developed. Strategies and techniques will be selected for field testing, of which the results will be discussed in the group. Positive approaches will be put in the Training Guideline. 
The capacity building of the research group members will be measured and monitored through group discussions and individual interviews with the researcher. Members are requested to keep a research log that will be used during the interviews.

\section{Group composition}

Because the training guideline should be usable in all departments and the DGE, each department and the DGE itself should delegate one member to the working group. This staff member should have experience in developing and conducting training and workshops. A mixture of deputy directors and office staff would be desirable, so staff members can learn from each other.

Working group members will come together on a regular basis (1-2 times a month) and discuss and decide which training techniques will be tested in workshops to come and decide who and when will observe group members during their conducting of a training and workshops. A relatively small number of training and workshops will be observed by other members. Results are discussed and placed in the guideline.

\section{The results of the research}

The research will result in four products:

1. a training guideline usable for the DGE and its departments;

2. the raised capacity of the research group members;

3. an instrument to use the knowledge and skills of the local experts (working together as a group) for the development of formal policy or products like this training guidelines;

4. a starting point for the training on project management cycle.

Jan Berkvens,

capacity building and educational adviser, Directorate General of Education, MoEYS.

janberkvens@gmail.com

092-945910. 


\section{ANNEXE 4 \\ First interview questionnaire}

First interview with research group members

name:

department:

sex $\mathrm{m} / \mathrm{f}$ date of birth:

highest education:

studied abroad y / $\mathrm{n}$

kind of study:

works at MoEYS since:

have had training on tw formal $\quad \mathrm{y} / \mathrm{n}$ year: where:

experiences:

have had training alongside consultant $\mathrm{y} / \mathrm{n}$ year: where:

experiences:

develops training $\quad \mathrm{y} / \mathrm{n}$ how often: topics:

delivers training $\quad \mathrm{y} / \mathrm{n}$ how often: topics:

previous work:

have had training on tw formal $\quad \mathrm{y} / \mathrm{n}$ year: where:

have had training alongside consultant y / $\mathrm{n}$ year: where:

develops training $\quad \mathrm{y} / \mathrm{n}$ how often:

topics:

delivers training $\quad \mathrm{y} / \mathrm{n}$ how often:

topics:

possess research sk $\mathrm{y} / \mathrm{n}$ on:

expectations of this project: professional / personal 
What would member like to learn?

\begin{tabular}{|l|l|l|l|l|l|}
\hline & & 4 & 3 & 2 & 1 \\
\hline 1 & How important is a training guideline to you & $\begin{array}{l}\text { very } \\
\text { good }\end{array}$ & average & $\begin{array}{l}\text { a little/ } \\
\text { not so } \\
\text { good }\end{array}$ & $\begin{array}{l}\text { not/ } \\
\text { good at } \\
\text { all }\end{array}$ \\
\hline 2 & How is your knowledge on how to develop tw & & & & \\
\hline 3 & How are your skills on how to develop tw & & & & \\
\hline 4 & $\begin{array}{l}\text { How are your k/s compared to your colleagues } \\
\text { on t/w }\end{array}$ & & & & \\
\hline 5 & How is your knowledge on how to deliver tw & & & & \\
\hline 6 & How are your skills on how to deliver tw & & & & \\
\hline 7 & How are your k/s compared to your colleagues & & & & \\
\hline 8 & $\begin{array}{l}\text { How do you rate yourself as a training } \\
\text { developer? }\end{array}$ & & & & \\
\hline 9 & How do you rate yourself as a training leader? & & & & \\
\hline 10 & $\begin{array}{l}\text { How do you rate yourself for monitoring } \\
\text { training? }\end{array}$ & & & & \\
\hline 11 & $\begin{array}{l}\text { How do you rate yourself for evaluating } \\
\text { training? }\end{array}$ & & & & \\
\hline 12 & $\begin{array}{l}\text { How do you rate yourself for follow up on } \\
\text { training? }\end{array}$ & & & & \\
\hline
\end{tabular}

The most successful training

Think about one of your most successfull trainings / workshops conducted and answer the following questions:

1. What was the training about?]

2. How many trainers and participants were there?

3. How long was the training?

4. Who and how was the training developed?

5. What kind of activities took place?
6. How was the training monitored?

7. How was the training evaluated?

8. Was there follow up? If so, how was that provided?

9. What made the training so successful?

The least successful training

Think about one of your least successful trainings / workshops conducted and answer the following questions:

1. What was the training about?

2. How many trainers and participants were there?

3. How long was the training?

4. Who and how was the training developed?

5. What kind of activities took place?
6. How was the training monitored?

7. How was the training evaluated?

8. Was there follow up? If so, how was that provided?

9. What made the training so successful? 
Training on training

Did you have training on how to develop and conduct training? $\quad \mathrm{y} / \mathrm{n}$

If so, please answer the following open questions.

Participants' reactions:

1. Did you liked the training? Why?

2. What kinds of materials were provided?

3. Where was the trainer from (foreign consultant or Cambodian?

4. Was she / he knowledgeable and helpful during the training?

Participants' learning:

5. What did you learn about training and workshops?

6. How was the learning assessed?

7. What made the assessments good?

Participants' use of new skills and knowledge:

8. What of the knowledge/skills did you put into your work and how?

9. What prevented you from implementing the new knowledge and skills in your work?

10. What would you still like to put into your work?

Support and change:

11. How was the follow up arranged of the training?

12. How were you supported and by whom?

13. Was the trainer helpful after the training? In what way?

14. What was limiting for implementation of new skills and knowledge?

15. What was successful for implementation of new skills and knowledge?

Students (participants' participants) improvements:

16. How did/do your measure the improvements of your participants in one of your trainings?

17. How did your new knowledge and skills influence participants' learning in trainings and workshops you give? 


\section{ANNeXE 5}

\section{First interviews - successful and unsuccessful trainings}

\begin{tabular}{|c|c|}
\hline Most successful & Successful because \\
\hline $\begin{array}{l}\text { Group member } 1 \\
\text { Diarrhea } \\
\text { prevention } 1997\end{array}$ & $\begin{array}{l}\text { started with small survey before } \\
\text { start (pre measure) } \\
\text { post test: compared so result known } \\
\text { change in knowledge/skills/ } \\
\text { behavior. Effect on children was } \\
\text { good to see }\end{array}$ \\
\hline $\begin{array}{l}\text { Group member } 2 \\
\text { Textbook } \\
\text { distribution }\end{array}$ & $\begin{array}{l}\text { monitored } \\
\text { knowledge was taken and just in } \\
\text { provinces } \\
\text { trained trainers and monitored } \\
\text { them } \\
\text { trainers did good }\end{array}$ \\
\hline $\begin{array}{l}\text { Group member } 3 \\
\text { Community } \\
\text { preschool } \\
\text { milestone }\end{array}$ & $\begin{array}{l}\text { no questions at the end by trainees, } \\
\text { look at their happy faces } \\
\text { activities did make people } \\
\text { understand } \\
\text { during monitoring effect was good: } \\
\text { in field happened } \\
\text { POE was involved }\end{array}$ \\
\hline $\begin{array}{l}\text { Group member } 4 \\
\text { School director } \\
\text { training on gender } \\
\text { and motivation } \\
2006\end{array}$ & $\begin{array}{l}\text { the participants can practise and do } \\
\text { (use the knowledge in skills, jan) }\end{array}$ \\
\hline
\end{tabular}

\begin{tabular}{|l}
\hline Activities \\
$\begin{array}{l}\text { different methodologies: } \\
\text { brainstorming, roleplay, present } \\
\text { and show activities in diff ways, } \\
\text { Q\&A, real practise }\end{array}$ \\
$\begin{array}{l}\text { group activites } \\
\text { theory } \\
\text { corrections on the group activity } \\
\text { sheets }\end{array}$ \\
a lot of activities, learned by TA \\
sit together and share, by \\
presentation. \\
make questionnaire, group \\
discussion, \\
some games in between
\end{tabular}

Monitored
directly during training, summary
and evaluation at the end of every
day, post test after training, show
objectives, goals, schedule at the
beginning, pretest. field monitor
during the last training day a
provincial plan was made by each
province collected and bundled in
one document. follow up was
provided based on this document
during: identify people with weak
understanding
after: see how they teach in the field
and give recommendations
discuss results back in department
no form
observe group discussion, identify
who does not share ideas and ask
them to share.
go to school after 1 month observe
classroom management, lessonplan,
motivation, room organisation.

\section{Evaluated}

pretest and posttest comparing

by feedback from participants

evaluation form on duration

content, trainer, food, accomodation

and travel

monitoring on effect

every training day form (i did not see that)

at the end of the course

give recommendations during

sit together with trainers and

discuss difficulties and questions a

trainer could not answer. monitoring and discuss results at dep.

\section{Follow up}

depends on the problem after

eldmonitor: refreshment training shop, adjusted program for new groups

appointments by province $2-4$

people went for monitoring 2

we go to help them when something is not good. 


\begin{tabular}{l|l}
$\begin{array}{l}\text { Most successful } \\
\text { Group member } 5\end{array}$ & $\begin{array}{l}\text { Successful because } \\
\text { I know a lot about the topic so I } \\
\text { could explain easy. I only facilitated } \\
\text { the part of the training I know a lot } \\
\text { about. } \\
2006 \text { and reentry } \\
\text { program 2006 }\end{array}$ \\
\hline $\begin{array}{l}\text { Group member 6 } \\
\text { School } \\
\text { development plan } \\
\text { provinces 2006 }\end{array}$ & $\begin{array}{l}\text { Participants were interested in the } \\
\text { theory because it is about their own } \\
\text { work and they were more active } \\
\text { during all activities. }\end{array}$ \\
\hline $\begin{array}{l}\text { Group member 7 } \\
\text { Action research }\end{array}$ & $\begin{array}{l}\text { participants apply the knowledge } \\
\text { they learned in their current work. } \\
\text { They implemented knowledge and } \\
\text { skills. }\end{array}$ \\
\hline
\end{tabular}

\section{Activities}

breaking eye (icebreaker, jan).

select 5 trainees to lead the

workshop groups to collect

information.
group discussion with facilitator in

each group.

andouts spread with information.

explain content, asking participants what they want to know, group discussion, presentations, ask questions, trainer summarizes and gives group idea, closing ceremony. Sok Yean gives different ways to split the participants in groups: based on 12-3-4, province, district, technical groups eso

group discussion: participants present their ideas.

commitment to apply the results of

the training in their work.

\begin{tabular}{l} 
Monitored \\
with evaluation paper (gap of \\
understanding, jan) \\
one year later, to see what is put \\
into the work of the participants \\
based on workshop. questionnaire \\
and obs \\
\hline than Soeun explains about \\
evaluation form
\end{tabular}

\section{Evaluated}

with evaluation paper (gap of

understanding, jan) evaluation sheet, BUT she

explained that the sheet normally is

given at the beginning of the

training to give participants the

opportunity to evaluate all parts of

the training directly. She said the

form has questions on each part of

the training I need to check this,

because I did not see something like

that in Cambodia before. Here

computer was broken, so we could

not see now.

the evaluation form was classified

to activities, content, expectatio
the results by the participant.
Follow up

done after the evaluation. if people have any problem, they could ask or write an anonymous letter when they are afraid (not asked, but how to explain to this peron when you don't know who it is?) or is it only used for improving the training and provide additional training later on? she is not sure about what follow up is. We will work on that during one of the next meetings of the TGWG

not using a form, it was informal follow up: we see and experience the difference in the work of the people: before they were passive do-ers after the training they became active. 


\begin{tabular}{|c|c|c|c|c|c|}
\hline Most successful & Successful because & Activities & Monitored & Evaluated & Follow up \\
\hline $\begin{array}{l}\text { Group member } 8 \\
\text { In general }\end{array}$ & All our trainings are successful & $\begin{array}{l}\text { 1. study visit in the field for training } \\
\text { like PTA. } \\
\text { 2. group working, like group } \\
\text { discussion. participants can raise } \\
\text { their ideas they can say their } \\
\text { problems. The steps we take are: } \\
\text { - we give them big paper flipchart, } \\
\text { jan) they write their problems or } \\
\text { issue each group. } \\
\text { - put on the wall and brief the big } \\
\text { group to which problem is the same } \\
\text { for schools } \\
\text { - discuss the problem and how to } \\
\text { solve it. sometimes schools or } \\
\text { parents have different solutions that } \\
\text { could hep schools with problems. } \\
\text { - they share experiences, if another } \\
\text { school resolved or parents resolved } \\
\text { (learn from each other). Some } \\
\text { villages for instance use child } \\
\text { networks, or school campaigns, or } \\
\text { go to the house and explain } \\
\text { importance of school. } \\
\text { - we ask the schools to take } \\
\text { experience from others and if they } \\
\text { can use / follow up. we ask them in } \\
\text { writing how they want to do } \\
\text {-if they can not do something, we } \\
\text { help them to find a solution. }\end{array}$ & $\begin{array}{l}\text { (Borah is not clear on the difference } \\
\text { between monitoring, evaluation } \\
\text { and follow up. He uses the terms } \\
\text { incorrectly and inconsistently.) } \\
\text { for some trainings, like the pta, we } \\
\text { do not know if they follow up or } \\
\text { not (we do not know if the } \\
\text { participants implement the } \\
\text { solutions they found during the } \\
\text { training, jan). } \\
\text { For other trainings we do goo into } \\
\text { the province with POE and DOE } \\
\text { and we visit schools together. In } \\
\text { some cases we ask the school } \\
\text { director what has been done based } \\
\text { on the training and if not why not. } \\
\text { In other cases we just go and look in } \\
\text { the class. Before we never looked at } \\
\text { the training goals (and objectives, } \\
\text { jan) we just went to follow up } \\
\text { (meant is monitoring, jan) to see if } \\
\text { the workshop ideas were used or } \\
\text { not. }\end{array}$ & $\begin{array}{l}\text { we never evaluate long term, so } \\
\text { after the workshop. we only do } \\
\text { during. In some cases we only do } \\
\text { by observation during the } \\
\text { workshop. There are no evaluation } \\
\text { forms available for all trainings. }\end{array}$ & $\begin{array}{l}\text { After some workshops we do not } \\
\text { know if participants implement } \\
\text { what they learned, because we do } \\
\text { not go and see. } \\
\text { other trainings we follow up } \\
\text { (monitor, jan) the activities we gave } \\
\text { them this makes clear that for some } \\
\text { ws or training activities for } \\
\text { implementation are given to the } \\
\text { participants. } \\
\text { But, Borah says, every training } \\
\text { should have follow up (and } \\
\text { monitoring and evaluation, jan.) }\end{array}$ \\
\hline $\begin{array}{l}\text { Group member } 9 \\
\text { Staff development } \\
\text { unesco } 2007\end{array}$ & $\begin{array}{l}\text { the subject is the key to develop the } \\
\text { people to do a better job }\end{array}$ & $\begin{array}{l}\text { discussions to exchange experience and } \\
\text { we get new ideas from others. } \\
\text { brainstorm? (I think brainstorm and } \\
\text { icebreaker are the same, is that so? I } \\
\text { forget about the methodology name } \\
\text { and activities) }\end{array}$ & $\begin{array}{l}\text { pretest and posttest and we ask them } \\
\text { the extra things they learned but we did } \\
\text { not put in pre and posttest (this is } \\
\text { more evaluation than monitoring, } \\
\text { jan) } \\
\text { (doing monitoring after the training } \\
\text { in the field I do not do. THIS IS } \\
\text { PECULIAR, BECAUSE HE DOES } \\
\text { FOLLOW UP, JAN) }\end{array}$ & $\begin{array}{l}\text { (after a long time thinking:) the } \\
\text { training course is successful if we have } \\
\text { enough material, participants joing } \\
\text { every day and the pp we want to have } \\
\text { in the workshop join. there needs to be } \\
\text { enough money. (these are more } \\
\text { criteria before we start, and do not } \\
\text { guarantee good evaluation or good } \\
\text { result.concept unclear? }\end{array}$ & $\begin{array}{l}\text { to trainee: last year I do } 2 \times 24 \text { provinces } \\
\text { for JFPR. we give help when we see that } \\
\text { they cannot do (they are the } \\
\text { participants, jan) }\end{array}$ \\
\hline
\end{tabular}




\begin{tabular}{|c|c|c|c|c|c|}
\hline Least successful & Successful because & Activities & Monitored & Evaluated & Follow up \\
\hline $\begin{array}{l}\text { Group member } 1 \\
\text { Deng } \\
\text { hemmoragique } \\
\text { fever } 1998\end{array}$ & $\begin{array}{l}\text { not enough support to do (budget) } \\
\text { no certificate aso }\end{array}$ & multi method & $\begin{array}{l}\text { not enough material for doing all } \\
\text { activities during the training }\end{array}$ & $\begin{array}{l}\text { lack of documents and material to } \\
\text { do so. we did not follow the } \\
\text { workplan so small effect }\end{array}$ & no budget \\
\hline $\begin{array}{l}\text { Group member } 2 \\
\text { Teaching aid } \\
\text { material grade 1-7 }\end{array}$ & $\begin{array}{l}\text { no time to practise, not enough } \\
\text { material to produce learning aids, } \\
\text { no budget for providing training to } \\
\text { schools, schools received the theory } \\
\text { only, they did not practise. no } \\
\text { transfer and no creativity }\end{array}$ & $\begin{array}{l}\text { theory on how to produce teaching } \\
\text { aids and discussing. some pictures } \\
\text { were drawn }\end{array}$ & $\begin{array}{l}\text { plan for } 2007 \text { but no money } \\
\text { provided to do }\end{array}$ & no time no money & want to, but no budget \\
\hline $\begin{array}{l}\text { Group member } 4 \\
\text { Not specific - in } \\
\text { general }\end{array}$ & $\begin{array}{l}\text { Students are lazy sometimes } \\
\text { no money to buy materials for } \\
\text { training }\end{array}$ & $\begin{array}{l}\text { some participants do not like group } \\
\text { discussion } \\
\text { many presentations and trainings } \\
\text { are like a lecture: too long talking to } \\
\text { participants: short talking! }\end{array}$ & No & No & No \\
\hline
\end{tabular}




\begin{tabular}{|c|c|}
\hline Least successful & Successful because \\
\hline $\begin{array}{l}\text { Group member } 6 \\
\text { Gender training } \\
2006\end{array}$ & $\begin{array}{l}\text { participants were interested but } \\
\text { practising result not so good like } \\
\text { expectations. Demends on how } \\
\text { people think in society about } \\
\text { gender. We want to change attitude } \\
\text { of people, but very difficult. goes } \\
\text { very slow. but better than before. } \\
\text { Especially difficult to change some } \\
\text { male leaders. But Sok Yean is very } \\
\text { happy with this work: education is } \\
\text { very important to create change. }\end{array}$ \\
\hline $\begin{array}{l}\text { Group member } 7 \\
\text { In general }\end{array}$ & $\begin{array}{l}\text { a training is not successful if } \\
\text { trainees do not use the knowledge } \\
\text { and skills they learn in the training. } \\
\text { Sometimes they do not know about } \\
\text { the training topic. }\end{array}$ \\
\hline $\begin{array}{l}\text { Group member } 8 \\
\text { In general }\end{array}$ & \\
\hline $\begin{array}{l}\text { Group member } 9 \\
\text { In general }\end{array}$ & - \\
\hline
\end{tabular}

\section{Activities}

ime needed and demands of other duties should be weighed agains

each other. leaders of workshops

have other jobs and teachers can not

be called away from classroom long time.

we should notify people at least two weeks in advance, now we do not because of bad materials and

planning management.

people only attend if they get

money. this is bad attitude, because they also get new knowledge and skills. better to give people in other ways.

if a trainer does not share his lessons learned, not give any new ideas to participants.

a trainer does not know about the opic.

if a training is not participatory. if a trainer and facilitator do not discuss their work.

if the trainee is not concentrated.

if the training topic is not relevant to

the participant, for instance primary

teachers learning about

management.

not successful activities are does that re not prepared well. If we follow the prepared sch people responsible for a topic do good, the training is a topic do During one works

roup dise work

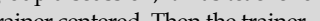
akd: "any question?" were not any. So he continued to he next part. But there were no questions because nobody understood. Not successful.

lecturing is not successful. only the trainers talks one way, no reply from participants. they need to share from group to group instend. Good number of participants for group discussion

\section{Monitored}

No

Evaluated

No

evaluations should be done by topic and session, so the facilitator and trainer can discuss during breaks, before starting another session.

last ferw years, sometimes the difficultic because we did not have time to visit all provinces

Follow up

not clear on follow up

we always do successful 


\section{What did you learn on training?}

1 New methodology, learning by doing

2 learn by doing is important

facilitate, not only speak and speak

provide enough activities
try being child / participant centered.

Use activities, action plan

Use activities

Training in Japan on training

Learned how to make clear objectives

materials included handouts, video, how to use overhead (not too much on one slide, using bullet points, rest you explain.

used mindmapping for easy to remember.

group discussion should be with 5-6 people not more.

the learning was assessed by presentation of learned, and filling out an evaluation form.

After coming back, she shared the information with her colleagues and director what happened after that?!)

Reasons not to implement the gained knowledge and skills: too big rooms, too many people, lack of good facilities, other limitations (knowledge, skills, budget, jan)

1. plan

train myself and other trainers (based on previous experience)

material preparation

target group

level of target group (last time different knowledge in the group: we invited teachers and community members, but for community members you need to lower the level in order to let them understand. technical words are unknown to them)

6. decide on time duration.

10 That we need to plan like a lesson plan with times, so much for this, so much for that. 


\section{ANNEXE 6}

\section{Final intervention questionnaire}

Final Intervention evaluation Questionnaire

Date: Time:
Name:
Location:
Thank you for your time to engage in this interview. I would like to ask you some questions about the Training Guideline
Working Group you participated in from January to August 2007. We developed the Guidelines that were recently approve
by the Ministry. As you know, the way the working group worked together, is at the same time my PhD research. I would
like you to answer all the questions as honest as possible. Even if you are afraid I will not be really happy with the answer,
please give the honest answer. I am way happier with an honest hg nanswer than an answer that would flaw my research
Also, if a question is not completely clear to you, feel free to ask me to repeat or restate the question.
This interview will approximately take 1 hour. It contains questions about the 5 levels of Professional Development
Evaluation of Guskey:
1. Participants' reactions;
2. Participants' learning;
3. Organisation support and change;
4. Participants' use of new knowledge and skills;
5. Student learning outcomes.
The data of this interview will only be used for my PhD research and only through my thesis shared with other persons.

Now let's start the interview.

\section{Participants' reactions}

The first couple of questions are about your feeling about the way we worked on the training guideline.

1. Did you like working on the TG?

2. What aspects did you like about working on the TG?

3. How do you think working on the TG was useful for you?

4. What did you enjoy most about working on the TG?

5. How do you feel about the way the work on the TG was facilitated?

6. How do you feel about the content of the meetings?

7. How do you feel about the activities we did?

8. How do you feel about the special meeting at TPO?

9. How do you feel about the materials?

10. Was the time suitable for you?

11. And the place?

\section{Participants' learning}

The next questions are about what you learned of the work we did on the training guideline.

12. What new things do you feel you learned?

There were different topics we worked on. Could you say for each what you learned from it? 
13. How to develop a checklist (the steps we made)

14. Facilitate

15. Facilitate discussions
16. Participatory techniques

17. Evaluation forms

18. Ice breakers / energizers

19. Monitoring/evaluation/follow up

Organization support and change

The next questions are about how the organisation supported the introduction of what you learned during the working group meetings in your daily work.

20. What changed in your department during or after working in the working group?

21. How did working in the working group affect the cooperation / working together in your department?

22. And working together with other departments?

23. How was what you tried to change supported in your department?

24. What could the management change more to support you better?

Participants' use of new knowledge and skills

The next questions are about how you used the obtained knowledge and skills you gained in your daily work.

25. What did you want to change after working in the working group?

26. How did you experiment with the knowledge and skills you gained in your daily work?

27. Could you share one example of what you tried?

28. What went less?

29. What went well?

30. What would you do differently?

Student learning outcomes

The next questions are about what participants of the training you just mentioned felt about the new approach and what they learned from it, in your opinion.

31. How do you feel your new approach affected the participants learning?

32. How do you feel your new approach affected the participants well being?

33. How do you feel your new approach influenced the attention of the participants? 


\title{
ANNEXE 7
}

\section{List of analysis codes}

\author{
CP - CAMBODIAN SPECIFIC PRE-REQUISITES \\ HISTORY \\ CP-FO French colonisation \\ CP-CW Civil war \\ CP-KR Khmer rouge \\ CP-OC Occupation \\ CULTURE \\ C-IC \\ C-PD \\ individualism/collectivism: corruption / responsibility taking / blaming \\ power distance: hierarchy / respect / compliance \\ C-UA \\ C-MF \\ uncertainty avoidance: loss of face / timid / implicit / shyness / seeking appr \\ masculine/feminine: self-performance \\ C-LS long/short term: ambition / short term thinking / critical thinking \\ CURRENT SITUATION \\ CS-DON Donor influence: brain drain \\ CS-SAL Low salaries: per diem \\ CS-K\&S Lack of knowledge \& skills \\ CS-LANG Language barrier \\ CS-NOSHARE No common / shared practise. \\ CURRICULAR SPIDER WEB \\ CUR-A\&O Aims and objectives \\ CUR-CONT Content \\ Definitions \\ Concepts \\ CUR-LACT Learning activities \\ Sharing \\ CUR-TROLE Teacher role \\ CUR-MAT Materials (lack of money) \\ CUR-GROUP Grouping \\ CUR-ROOM Location \\ CUR-TIME Time (constraints) \\ CUR-PROLE Participants role \\ Group support \\ Peer learning / support
}




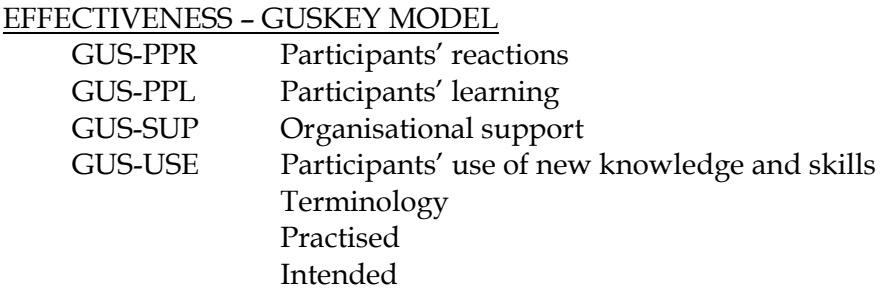

GUS-BEN Secondary beneficiaries' learning outcomes

EFFECTIVENESS - UNANTICIPATED

UN-COM Communication

UN-REL Relationships

UN-SHARE Sharing

\section{ENTYMOLOGY}

$\begin{array}{lll}\text { I } & \text { Importance } \\ \text { C } & \text { Clarity } \\ \text { G } & \text { Good } \\ \text { H } & \text { Hopeful }\end{array}$




\section{ANNEXE 8 \\ Short-term evaluation form first and second prototypes}

Short term evaluation form - first draft 01/02/2007

\begin{tabular}{|l|l|l|l|l|l|}
\hline & Excellent & Good & Fair & Poor & Comments \\
\hline 1. content & & & & & \\
\hline $\begin{array}{l}\text { 2. } \text { - durganisation } \\
\text { - time } \\
\text { - location }\end{array}$ & & & & & \\
\hline 3. trainers / facilitators & & & & & \\
\hline 4. Materials & & & & & \\
\hline 5. Refreshment & & & & & \\
\hline 6. Per diem & & & & & \\
\hline
\end{tabular}

7. Commitment:

8. Suggestion:

Short term evaluation form - second draft 06/02/2007

I

1. Are you interested in this training organisation? Why?

2. Does the training spend suitable time for you? Why?

3. Is it suitable place for you? Why?

4. Is the training course useful for your Department? What?

5. What is the advantage and the disadvantage of good leader?

6. How is about the refreshment?

II

1. What subject is the most important for your Department?

2. Which subject is the least important for your Department?

3. What subject don't you understand?

III

1. What is the new experience you get from this training?

2. What will you do after this training? 


\section{ANNEXE 9 \\ Long-term evaluation form first and second prototypes}

Long Term evaluation form - first draft 01/02/2007

1. What is the advantage of the workshop for you?

2. Do you get the new knowledge from training course to disseminate, who and how many?

3. Until now, can you follow the theories of the training course? If yes, what are they? If no, why?

4. Do the contents of the training course answer to your needs?

5. Do target groups have any attitude/behaviour change? \%?

6. Do you have strategies to improve next step?

Long term evaluation form - second draft

I Participants' learning

1. Does the content of the training course answer to your needs?

2. Do you have strategies to improve next steps?

3. What is the advantage of the training?

II Organisation support and change

1. What does your organisation get from the training?

2. Are you used to make report to your Director?

3. Does your Department follow-up your implementation?

4. Does he make some recommendation?

5. Can the problems be solved?

III Participants' use of new knowledge and skills

1. Do you get the new knowledge from training course to disseminate? Who, and how many?

2. Until now, can you follow the theories of the training? If yes, what are they? If no, why not?

3. Do target groups have any attitude/behaviour change? 


\title{
ANNEXE 10
}

\section{Short-term evaluation form final version}

\author{
Short Term Training Evaluation Form
}

Topic:

Date:

Department: Position: Name (optional):

Directions: Listed below are questions about the training you just followed. Please answer the questions as honest as possible. The information gathered with this form will be used to improve the content and design of future training and workshops.

\section{About the organization of the training/workshop}

Please choose the best answer, matching your opinion. Tick the box using ' $X$ '.

\begin{tabular}{|c|c|c|c|c|c|}
\hline & Item & Excellent & Good & Fair & Poor \\
\hline 1 & How was the training organized? & & & & \\
\hline 2 & How was the time for training used? & & & & \\
\hline 3 & $\begin{array}{l}\text { How was the location of the training for } \\
\text { you? }\end{array}$ & & & & \\
\hline 4 & $\begin{array}{l}\text { How useful was the training for your } \\
\text { department }\end{array}$ & & & & \\
\hline 5 & How were the refreshments? & & & & \\
\hline 6 & $\begin{array}{l}\text { How was the use of different media } \\
\text { during the training? (use of flip charts, } \\
\text { overhead projector, lcd) }\end{array}$ & & & & \\
\hline 7 & How comfortable was the room? & & & & \\
\hline 8 & $\begin{array}{l}\text { How was the translation during the } \\
\text { training? (if applicable) }\end{array}$ & & & & \\
\hline
\end{tabular}


About the content of the training/ workshop

Please choose the best answer, yes or no, and give the reason in the last column.

\begin{tabular}{|c|c|c|c|c|}
\hline & Item & Yes & No & If yes/no, please give reason \\
\hline 9 & $\begin{array}{l}\text { Did the training content match with the } \\
\text { course objectives? }\end{array}$ & & & \\
\hline 10 & Did the training match to your needs? & & & \\
\hline 11 & Was the training relevant for you? & & & \\
\hline 12 & Was the topic important to you? & & & \\
\hline 13 & Did you understand the topics? & & & \\
\hline 14 & $\begin{array}{l}\text { Did you get new knowledge from this } \\
\text { training? }\end{array}$ & & & \\
\hline 15 & $\begin{array}{l}\text { Did you get new skills from this } \\
\text { training? }\end{array}$ & & & \\
\hline 16 & $\begin{array}{l}\text { Did you practice new skills during this } \\
\text { training? }\end{array}$ & & & \\
\hline 17 & Did the trainers follow the schedule? & & & \\
\hline 18 & $\begin{array}{l}\text { Did the trainers communicate well with } \\
\text { the participants? }\end{array}$ & & & \\
\hline 19 & $\begin{array}{l}\text { Did the trainers provide clear } \\
\text { explanations? }\end{array}$ & & & \\
\hline 20 & $\begin{array}{l}\text { Did the trainers summarize at the end of } \\
\text { each topic? }\end{array}$ & & & \\
\hline 21 & $\begin{array}{l}\text { Did the trainers use the materials } \\
\text { effectively? }\end{array}$ & & & \\
\hline 22 & $\begin{array}{l}\text { Will technical support (follow-up) be } \\
\text { provided to you after the training? }\end{array}$ & & & \\
\hline
\end{tabular}

Please answer the following open questions.

23. What would you like to learn more about the topic?

24. What in the training should be improved?

25. You learned new knowledge and skills during this training. What of this knowledge and skills are you going to use in your daily work? 


\title{
ANNEXE 11
}

\section{Long-term evaluation form final version}

\author{
Long Term Training Evaluation Form
}

Topic:

Date:

Department: $\quad$ Position: Name (optional):

Directions: Listed below are questions about the training or workshop on ...... you followed a while ago. Please answer the questions as honest as possible. The information gathered with this form will be used to improve the content and design of future training and workshops.

\section{About the content of the training/ workshop}

Below you find the learning objectives of the training you followed.

How were the learning objectives met? Please choose the best answer, matching your opinion. Tick the box using ' $X$ '.

\begin{tabular}{|l|l|l|l|l|l|}
\hline & Objectives & Excellent & Good & Fair & Poor \\
\hline 1 & & & & & \\
\hline 2 & & & & & \\
\hline 3 & & & & & \\
\hline 4 & & & & & \\
\hline 5 & & & & & \\
\hline 6 & & & & & \\
\hline
\end{tabular}


Support and change at the workplace

Please choose the best answer, matching your opinion. Tick the box using ' $X$ '.

\begin{tabular}{|c|c|c|c|c|}
\hline & Item & Yes & No & $\mathrm{N} / \mathrm{A}$ \\
\hline 7 & Did you write a report about the training to your director? & & & \\
\hline 8 & $\begin{array}{l}\text { Did your department follow up your implementation plan and/or } \\
\text { your recommendations? }\end{array}$ & & & \\
\hline 9 & $\begin{array}{l}\text { Did your director make some recommendations about your } \\
\text { implementation plan and/or your recommendations? }\end{array}$ & & & \\
\hline 10 & $\begin{array}{l}\text { Do you think that the knowledge and skills you learned during the } \\
\text { training could be used to improve the work of your department? }\end{array}$ & & & \\
\hline
\end{tabular}

$N / A=$ not applicable, use this category only if a question can not be answered at all

\section{About the use of new knowledge and skills}

Please answer the following open questions.

11. What are the advantages of the followed training for you? Please describe.

12. Did you disseminate new knowledge and skills from the training?

YES / NO If YES: what did you disseminate?

If YES: to how many people did you disseminate?

If YES: where do these people work in your department?

If NO: Why did you not disseminate?

13. Do you notice any change in behavior and attitude in your own work?

YES / NO If YES: What change did you notice?

14. Do you notice any change in behavior and attitude in the people you disseminated the knowledge to?

YES / NO If YES: What change did you notice?

15. What are you planning to change in the near future, about this topic?

Please describe.

16. Was the training followed up by the trainer or other participants?

YES / NO

17. Are you satisfied with the follow-up that was provided to you?

YES / NO Why / Why not? 


\section{ANNEXE 12}

\section{Evaluation, monitoring and follow-up framework}

\begin{tabular}{|l|l|l|} 
& Programme Start & Programme Termination \\
\hline Planning Phase & Implementation Phase & Post Programme Phase \\
\hline
\end{tabular}

Basic Questions for Monitoring

1. Are activities being implemented according to plan?

2. Who is benefiting from the plan?

3. Are the expected outcomes/outputs being developed?

4. Is the amount of benefits being delivered in the right

amount?

5. What changes, if any, should be made to implement better?
Basic Questions for Evaluation

1. To what degree has the objectives been achieved over time?

2. Is the project cost effective?

3. What impact has the training had upon the participants?

4. And on the daily work?

5. What decision should be taken on the programme?

We MONITOR to PERFECT implementation, to make sure the implementation goes well.

We EVALUATE to JUDGE the performance of the programme.

(SAEMEO-INNOTECH Course Handouts in: Appeal; Monitoring and evaluation of literacy and continuing education programmes, Practitioners' manual, Asia-Pacific programme of education for all, Unesco Principal Regional Office For Asia And The Pacific, Bangkok, 1999). 



\section{AnNeXe 13 \\ Flipchart 'paon roboch knjom'}

(My brother / sister)

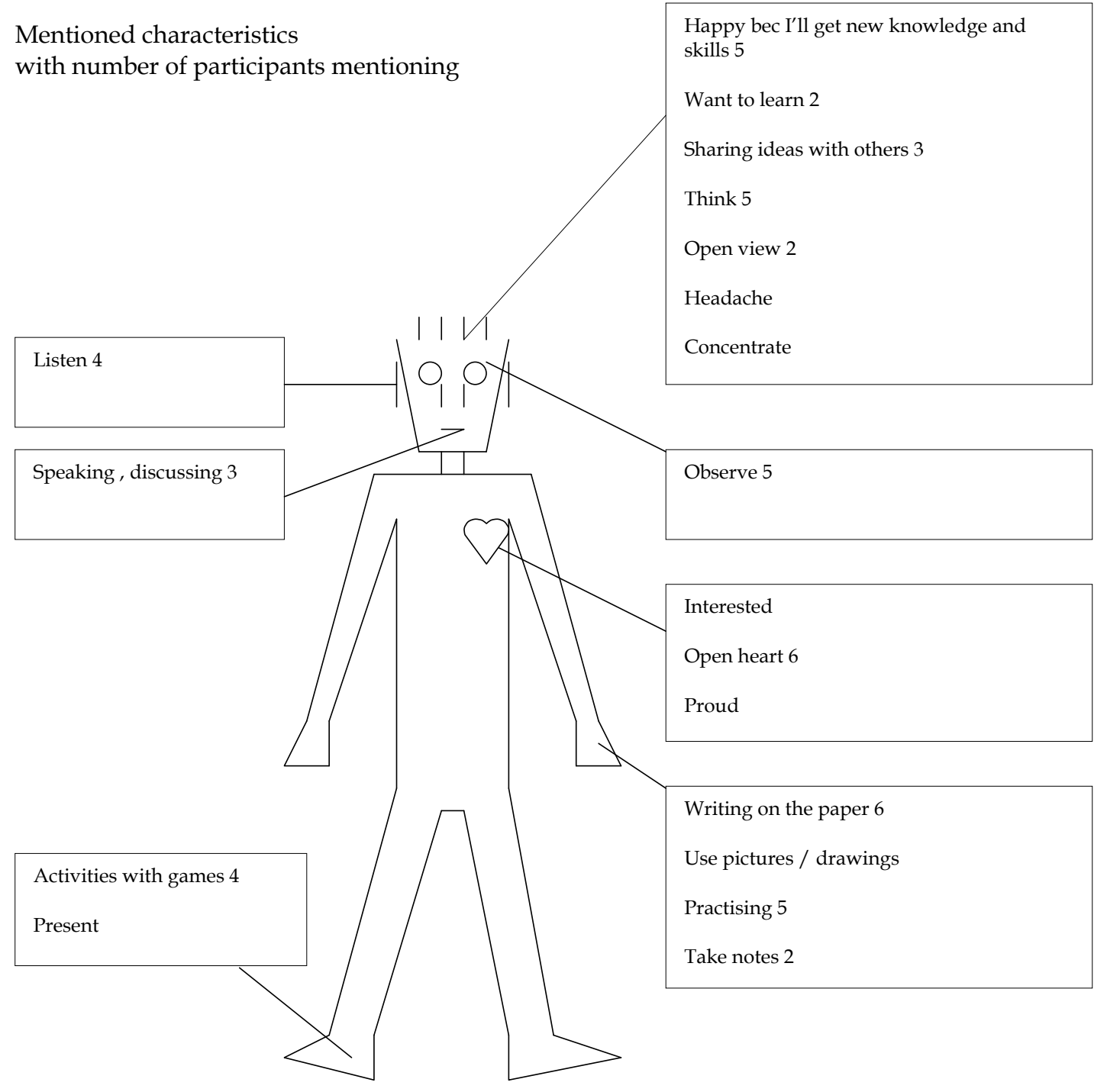




\section{ANNEXE 14 \\ Checklist 1 first prototype}

Results:

1. assignment from deputy director

2. budget planning, initial proposal to donors (based on annual plan)

3. selection of training topic

4. $\quad$ set goal and objectives

5. determine the target group of participants

6. select trainers and facilitators

7. discussion with trainer group

8. $\quad$ training needs assessment by trainers

9. discussion with trainer group on results of assessment

10. selection of activities

11. make a training schedule

12. decide on location, number of days, time

13. selection of training materials

14. budget description

15. planning evaluation

16. report writing

17. send to director

18. revision

19. send to director

20. submit to donor

21. first activity. 


\section{ANNEXE 15}

\section{Checklist 2 first prototype}

Flipchart: What should be prepared before and during training?

\begin{tabular}{|c|c|}
\hline before (yellow group) & before (pink group) \\
\hline $\begin{array}{l}\text { prepare power point and lcd } \\
\text { prepare forms of evaluations } \\
\text { prepare participants documents } \\
\text { prepare participants names lists } \\
\text { writing on the flipchart: } \\
\quad-\quad \text { objectives } \\
\text { - questionnaires } \\
\text { prepare lesson plan } \\
\text { set up responsible people } \\
\text { prepare the schedule } \\
\text { prepare teachin materials and flipcharts } \\
\text { prepare pretest } \\
\text { prepare venue }\end{array}$ & $\begin{array}{l}\text { prepare and schedule the workshop, with } \\
\text { agenda } \\
\text { preparing workshop materials for participants } \\
\text { copy documents for training } \\
\text { preparing workshop materials for training } \\
\text { prepare the place for workshop } \\
\text { checking and confirming the place } \\
\text { design participants list } \\
\text { invitation list and letter for HE and GE. } \\
\text { confirm the training to the participants } \\
\text { organizing the nomination letter of training } \\
\text { responsibilities } \\
\text { write department report }\end{array}$ \\
\hline
\end{tabular}




\begin{tabular}{|l|l|}
\hline during (yellow group) & during (pink group) \\
\hline $\begin{aligned} \text { opening ceremony } \\
\text { make the rule of the workshop: }\end{aligned} \quad$ morning and afternoon hours \\
$-\quad$ discipline & delivery training documents to participants \\
make a group list of participants & opening ceremony (national anthem) \\
provide group discussion & minutes of training (who) \\
$\begin{array}{l}\text { provide the activity game to wake up } \\
\text { participants }\end{array}$ & show the objectives of workshop \\
presentation of the topic & presentation of the topic \\
$\begin{array}{l}\text { provide post test (!) } \\
\text { summary ideas of group discussion }\end{array}$ & teabreak \\
$\begin{array}{l}\text { facilitator meeting at every the end of the day } \\
\text { (daily if multiple days) }\end{array}$ & games \\
$\begin{array}{l}\text { questions by participants (daily if multiple } \\
\text { days) }\end{array}$ & divide for group discussion, discuss \\
$\begin{array}{l}\text { workshop evaluation at the end } \\
\text { closing ceremony }\end{array}$ & sum up / summarize \\
\hline
\end{tabular}




\section{ANNEXE 16 Activity form}

\begin{tabular}{l}
$\begin{array}{l}\text { name of activity: } \\
\text { What's on my back?! }\end{array}$ \\
$\begin{array}{l}\text { objectives: } \\
\quad \text { - To activate people. } \\
\text { get energy after a break or serious session. }\end{array}$ \\
\hline lnumber of people: group of 4 or more \\
\hline time span: 6 minutes for one round \\
\hline materials needed: \\
$\quad$ - drawing of object or name of person on a piece of paper \\
\hline $\begin{array}{l}\text { Can be used for: } \\
\text { energizer }\end{array}$ \\
\hline $\begin{array}{l}\text { explanation / rules (including pictures): } \\
\text { Prepare a piece of paper with the drawing of an object or the name of a person. Make sure that } \\
\text { nobody can see. } \\
\text { Select one person and stick the piece of paper on the back of one person with tape. } \\
\text { The person shows the other participants his or her back. } \\
\text { The person with the paper on his or her back needs to find out what object or person is sticked } \\
\text { to his or her back. } \\
\text { Now the person with the paper on his/her back starts asking questions. The other participants } \\
\text { may only answer with YES or NO. } \\
\text { Giving hints is NOT allowed. } \\
\text { The game is over when the person has guessed the right object or name, or the time is over. } \\
\text { other: } \\
\text { The game could also be done with sticking a piece of paper on the back of all participants. The } \\
\text { one that guesses right is number } 1,2 \text { or } 3 .\end{array}$ \\
\hline
\end{tabular}




\section{ANNEXE 17 \\ Categorisation of energizers/icebreakers/wrap-up}

1. Energizers

- play games

- do exercise

- warm up

- after lecture

- short, funny

- concentration needed

- to relax after hard thinking

- happy

- role play

- after lunch or break

- song

2. Icebreakers

- introduce each other names

- tell organisation

- short joke or story

- game

- make participants happy

- short and powerful

- sing the song

3. Wrap upend of group work

- to share findings

- suggestion/recommendation

- summarize what is learned

- evaluate

- Q\&A

- learn from each other

- clearly understand group idea

- conclusion/ideas

- to find solutions

- presentation

- self made game 


\section{ANNEXE 18}

\section{Checklist final versions}

\section{Checklist 1 \\ PDA Development Phase}

If you performed a step, Tick the box: $\square$

1. $\square \quad$ Need for PDA (based on annual plan)

2. $\square \quad$ Select training topic

3. $\square \quad$ Set goal and objectives (Annexe 1)

4. $\square$ Budget planning, initial proposal to donors

5. $\square$ Determine target group of trainees/participants

6. $\square \quad$ Select trainers/facilitators

7. $\square$ Meeting with trainers/facilitator group

8. $\square \quad$ PDA needs assessment by trainers

9. $\square \quad$ Meeting with trainer/facilitator group on results result of assessment

10. $\square \quad$ Selection of activities by trainer/facilitator group

11. $\square \quad$ Selection of training materials

12. $\square \quad$ Participatory level check (Annexe 5)

13. $\square \quad$ Develop a PDA schedule (Annexe 3)

14. $\square \quad$ Develop pre and post test (if applicable) (Annexe 4)

15. $\square \quad$ Decide and arrange location, number of days, time

16. $\square \quad$ Budget description

17. $\square \quad$ Decide on monitoring and evaluation strategy

18. $\square \quad$ Decide on follow up strategy

19. $\square \quad$ Development Phase Check

20. $\square \quad$ Report/final proposal writing

21. $\square$ Send draft report/proposal to line manager

22. $\square \quad$ Revision of PDA document based on feedback from line manager

23. $\square \quad$ Send final report/proposal to line manager

24. $\square \quad$ Submit final report/proposal to donor

25. $\square \quad$ Revision of PDA document based on feedback from donor

26. $\square \quad$ Submit final report/proposal to line manager/donor

27. $\square \quad$ Approval by line manager and donor 


\section{Checklist 2 PDA Implementation Phase}

If you performed a step, Tick the box: $\square$

Two weeks to one week before

1. $\square$ Check location, time and days with location manager

2. $\square \quad$ Send out invitation letters

A few days before

3. $\square \quad$ Prepare flipcharts with objectives etc.

4. $\square \quad$ Prepare evaluation forms

5. $\square \quad$ Prepare teaching materials

6. $\square \quad$ Prepare hand outs

7. $\square \quad$ Prepare pre- and post-tests

8. $\square \quad$ Check and confirm location

9. $\square \quad$ Check and confirm participants

10. $\square \quad$ Prepare participants and per diem list

11. $\square \quad$ Arrange and prepare power point, LCD and back ups

The day before

12. $\square$ Check all materials (handouts, name list, badges, per diem)

13. $\square$ Check power point, LCD and back ups

14. $\square \quad$ Check and prepare the room (seating, registration etc.)

15. $\square \quad$ Arrange snacks and drinks

The day itself

16. $\square \quad$ Check all you checked the day before (12-15)

17. $\square$ Opening ceremony and National Anthem

18. $\square$ Give the rules: time, regular brakes, discipline etc.

19. $\square \quad$ Conduct pre test (if applicable)

20. $\square \quad$ Ask for commitment

21. $\square \quad$ Conduct the post test (if applicable)

22. $\square \quad$ Conduct the evaluation (short term)

23. $\square \quad$ Hand out per diem

24. $\square \quad$ Closing ceremony

25. $\quad \square \quad$ Trainer/facilitator meeting at the end of the PDA and keep notes of this meeting 


\section{Checklist 3 PDA End Phase}

\section{If you performed a step, Tick the box: $\square$}

The week after the PDA

1. $\square$ Write the trainer/facilitator discussion report

2. $\square$ Analyze the data from the Short Term Evaluation Forms

3. $\square$ Write the first report on the training

4. $\square \quad$ Discuss the report with the trainers/facilitators

5. $\square \quad$ Send the report to the line manager

6. $\square \quad$ Revise the report

7. $\square$ Revise the PDA

8. $\square \quad$ Send the report to the line manager and donor

9. $\square$ Revise the follow up activities plan

10. $\square \quad$ Start the follow up activities (until the Long Term Evaluation)

3 weeks to 6 months after the PDA

11. $\square$ Send the Long Term Evaluation Form to the participants and their managers

12. $\square$ Receive the Forms back at the office

13. $\square$ Analyze the data from the Long Term Evaluation Forms

14. $\square \quad$ Trainer/facilitator meeting on follow up results

15. $\square \quad$ Write the second report on training

16. $\square$ Discuss the findings and recommendations in the report

17. $\square \quad$ Revise the PDA or provide extra support and follow up to the participants

18. $\square \quad$ Send report to the line manager

19. $\square \quad$ Revise the report

20. $\square \quad$ Send the report to the line manager and donor 


\section{ANNEXE 19 \\ Definitions monitoring/evaluation/follow-up first prototype}

Monitoring

- We use monitoring when we finished providing training, after 2 or 3 months

- We monitor the participants that joined the training

- We monitor the knowledge of the participants

- We go to take the reports from provinces

- To want to know how activities are undertaken

- To see if activity implementation is appropriate to the policy of the Department or not

- To see if the activity has problem or not

- Make sure expenditure of budget is appropriate

- Control action plan

- To observe the performance of school directors and teachers

- Key definition: 'Monitoring is a process to observe / control progress performance of the process on objectives if they are functioning as planned and how to adjust'

Evaluation

- We use evaluation in the end of workshops, training, monitoring, finish of a program

- Find out the results of activities that the schools have done

- Compare the results with last year

- Annual result of courses

- To find out the weak and strong points of activities

- How much result is achieved

- It is to judge and recommend for future

- Key words for the definition are: Value, judging, end result, compare, recommendation

Follow up

- When we have the training provided

- Follow up on trainers

- Facilitate the training

- To observe how the training is going on

- Key words for the definitions: Giving support, to people, after training, support learning. 


\section{ANNEXE 20 \\ Definitions monitoring/evaluation/follow-up final version}

Definition Monitoring

Monitoring activities are all activities that are done to ensure that a process or program is implemented as planned.

Definition Evaluation

Evaluation is the judging of the result of a professional development activity, including the monitoring activities to get information for evaluation.

Definition Follow-up

Follow-up activities are all activities that are provided to support participants of professional development activities after a PDA. 


\section{ANNEXE 21}

\section{Training guideline schedule}

Schedule

\begin{tabular}{|c|c|c|c|c|c|}
\hline Part & What & How & Time & When & Who \\
\hline \multicolumn{6}{|c|}{ Day 1 Morning } \\
\hline & Opening ceremony & & 30 & $8-8.30$ & \\
\hline & Icebreaker & Throw the ball & 15 & $8.30-8.45$ & \\
\hline \multirow[t]{2}{*}{ I } & $\begin{array}{l}\text { Introduction/objectives } \\
\text { /rules }\end{array}$ & Introduction & 30 & $8.45-9.15$ & \\
\hline & $\begin{array}{l}\text { Theoretical background } \\
\text { definitions }\end{array}$ & $\begin{array}{l}\text { Brainstorm with different } \\
\text { coloured post its in small mixed } \\
\text { groups }\end{array}$ & $\begin{array}{l}120+ \\
15 \mathrm{~T} \\
\text { break }\end{array}$ & $9.15-11.30$ & \\
\hline \multicolumn{6}{|c|}{ Day 1 Afternoon } \\
\hline & Communication skills & $\begin{array}{l}\text { Role plays in small groups, } \\
\text { summarize and tips }\end{array}$ & 60 & $2-3$ & \\
\hline & Break & & 15 & $3-3.15$ & \\
\hline & Energizer & Connect the squares & 15 & $3.15-3.30$ & \\
\hline \multirow[t]{2}{*}{ II } & Introduction planning pahse & Introduction, checklist 1 & 30 & $3.30-4.00$ & \\
\hline & Smartie & $\begin{array}{l}\text { Small group discussions, } \\
\text { making objectives using } \\
\text { SMARTIE }\end{array}$ & 60 & $4.00-5.00$ & \\
\hline \multicolumn{6}{|c|}{ Day 2 Morning } \\
\hline & Energizer & Mirror game & 20 & $8.00-8.20$ & \\
\hline \multirow[t]{5}{*}{ II } & Learning Needs Assessment & $\begin{array}{l}\text { Small group discussion / } \\
\text { explanation wrap up }\end{array}$ & 30 & $8.20-8.50$ & \\
\hline & $\begin{array}{l}\text { PDA schedule and activities / } \\
\text { materials }\end{array}$ & $\begin{array}{l}\text { Small group discussion / } \\
\text { explanation wrap up }\end{array}$ & 40 & $8.50-9.30$ & \\
\hline & T break & & 15 & $9.30-9.45$ & \\
\hline & Pre- and post test & $\begin{array}{l}\text { Small group discussion / } \\
\text { explanation wrap up }\end{array}$ & 30 & $9.45-10.15$ & \\
\hline & $\begin{array}{l}\text { Participation and participatory } \\
\text { test }\end{array}$ & Activating session & 75 & $10.15-11.30$ & \\
\hline \multicolumn{6}{|c|}{ Day 2 Afternoon } \\
\hline & Energizer & "Throw the ...." & 15 & $2.00-2.15$ & \\
\hline & Evaluation and forms & $\begin{array}{l}\text { Small group discussion / } \\
\text { explanation wrap up }\end{array}$ & 30 & $2.15-2.45$ & \\
\hline & Planning of follow up & Lecture / problem tree & $\begin{array}{l}60+15 \\
\mathrm{~T} \\
\text { break }\end{array}$ & $2.45-4.00$ & \\
\hline & Writing project proposal & Lecture / discussion & 45 & $4.00-4.45$ & \\
\hline & Planning Phase evaluation & Presentation & 15 & $4.45-5.0-$ & \\
\hline
\end{tabular}




\begin{tabular}{|c|c|c|c|c|c|}
\hline Part & What & How & Time & When & Who \\
\hline \multicolumn{6}{|c|}{ Day 3 Morning } \\
\hline \multirow[t]{6}{*}{ II } & Energizer & 7-up! & 15 & $8.00-8.15$ & \\
\hline & Phase 2; implementation phase & Introduction & 30 & $8.15-8.45$ & \\
\hline & Checklist & $\begin{array}{l}\text { Small group discussion, } \\
\text { develop their checklists, } \\
\text { presentations }\end{array}$ & $\begin{array}{l}90+15 \\
\mathrm{~T} \\
\text { break }\end{array}$ & 10.30 & \\
\hline & & Explanation wrap up & 20 & $10.30-10.50$ & \\
\hline & Trainer/facilitator meeting & $\begin{array}{l}\text { Explanation / brainstorming in } \\
\text { small groups: What to do at the } \\
\text { end of the day? }\end{array}$ & 30 & $10.50-11.20$ & \\
\hline & Evaluation of phase 2 & Presentation & 10 & $11.20-11.30$ & \\
\hline \multicolumn{6}{|c|}{ Day 3 Afternoon } \\
\hline & Energizer & Connecting the dots & 20 & $2.00-2.20$ & \\
\hline \multirow[t]{4}{*}{ III } & Phase 3; end phase & Introduction & 30 & $2.20-2.50$ & \\
\hline & Evaluation & $\begin{array}{l}\text { Group discussion in two parts } \\
\text { in small groups: short term } \\
\text { (within } 1 \text { week) and long term } \\
\text { ( } 3 \text { weeks to } 6 \text { months) } \\
\text { Explanation wrap up }\end{array}$ & $\begin{array}{l}60+15 \\
\mathrm{~T} \\
\text { break }\end{array}$ & $2.50-4.05$ & \\
\hline & Evaluation & Lecture & 45 & $4.05-4.50$ & \\
\hline & Checklist 3 & Presentation & 10 & $4.50-5.00$ & \\
\hline \multicolumn{6}{|c|}{ Day 4 Morning } \\
\hline & Explanation this day activity & $\begin{array}{l}\text { Groups of max } 5 \text { prepare a } 1 \\
\text { day training useful for their } \\
\text { department following the } \\
\text { guidelines. It is the process they } \\
\text { should explain to the group } \\
\text { during the presentations, not so } \\
\text { much the content. }\end{array}$ & 15 & $8.00-8.15$ & \\
\hline & Working in groups & & 195 & $8.15-11.30$ & \\
\hline \multicolumn{6}{|c|}{ Day 4 Afternoon } \\
\hline & Presentations & $\begin{array}{l}\text { Each group presents to the big } \\
\text { group, each group has } 10 \\
\text { minutes to present the process } \\
\text { they followed }\end{array}$ & 120 & $2.00-4.00$ & \\
\hline & Evaluation & Short term evaluation form & 10 & $4.00-4.10$ & \\
\hline & Closing remarks & & 20 & $4.10-4.30$ & \\
\hline
\end{tabular}




\section{ANNEXE 22 \\ Certification letter}

Letter of Certificate for Master Trainer

To: Ms.

Phnom Penh, 2 September 2008

Dear Ms.,

It is an honour to write this letter to confirm that you were a valuable member of the Training Guideline Working Group, run by mr. Jan Berkvens, VSO, from December 2006 to March 2008. Your input in writing the Training Guideline for MoEYS has been significant.

During working in the Training Guideline Working Group and the Training Course to Government staff, you acquired strong knowledge and skills, you can use as a Master Trainer for Professional Development Activities (PDA's) like Training and Workshops.

We would like to thank you for your input and dedication towards this important programme.

With the highest regards,

Mr. Jan Berkvens MSc, capacity building adviser, VSO Cambodia.
Mr. Chea Vantha,

Education Programme Manager, VSO Cambodia. 


\section{ANNEXe 23 \\ 'The quest' list}

The Quest

Your team is to collect the following items

in 5 minutes

the first group finished is the winner

1. paperclip

2. computer chair (colour: grey)

3. the street number of the street running between street 118 and 136

4. aircon remote control

5. the handout of the first TGWG meeting

6. a pack of A4 paper
The Quest

Your team is to collect the following items

in 5 minutes

the first group finished is the winner

1. pen

2. broom (to clean the room)

3. 5 white flowers

4. 3 mobile phones of different brand

5. the street number of the street running between street 360 and 334

6. The handout of the third meeting. 


\section{ANNEXE 24}

\section{Participatory level check}

\begin{tabular}{|r|r|r|r|}
\hline & activities (description) & time(minutes) & time (minutes) \\
\hline 1 & Lecture & & \\
\hline 2 & Presentation by trainer/facilitator & & \\
\hline 3 & presentation by guest speaker & & \\
\hline 4 & reading & & \\
\hline 5 & disucssion in small groups & & \\
\hline 6 & group work & & \\
\hline 7 & individual work & & \\
\hline 8 & presentation by participants & & \\
\hline 9 & practising & & \\
\hline 10 & role play & & \\
\hline 11 & ice breaker & & \\
\hline 12 & wrap up & & \\
\hline 13 & & & \\
\hline 14 & Total amount of time & & \\
\hline & & & \\
\hline & participatory \% & & \\
\hline & & & \\
\hline & non-parcipatory \% & & \\
\hline & & & \\
\hline & & & \\
\hline & & & \\
\hline
\end{tabular}

to calculate the participatory $\%$ : Total of column 1

* 100 / total minutes

the non-participatory \%: Total of column 2

*100 / total minutes

The optimal \% of participatory time is between 40 and $60 \%$. If you are below this percentage, change some of the activities into more participatory ones. 
